\title{
New Precision Measurements of Deuteron Structure Function A(Q) at Low Momentum Transfer
}

\author{
by \\ Byungwuek Lee
}

Supervised by

Seonho Choi

\author{
A Dissertation Submitted to the Faculty \\ of Seoul National University \\ in Partial Fulfillment of the Requirements for \\ the Degree of Doctor of Philosophy
}

August 2009

Department of Physics and Astronomy

Graduate School

Seoul National University 



\section{Abstract}

Differences between previous measurements of low momentum transfer electrondeuteron elastic scattering prevent a clean determination of even the sign of the leading low momentum transfer relativistic corrections, or of the convergence of chiral perturbation theory. We have attempted to resolve this issue with a new high-precision measurement in Jefferson Lab Hall A. Elastic electron scattering was measured on targets of tantalum, carbon, hydrogen, and deuterium at beam energy of $685 \mathrm{MeV}$. The additional targets provide both kinematic calibration information, and tests of the precision of determining cross sections. The four-momentum transfer covered the range of $0.15-0.7 \mathrm{GeV}$. The experiment included a new beam calorimeter, to better calibrate the low beam currents used in the experiment, and new collimators to better define the spectrometer solid angles.

We obtained cross sections of deuteron as ratios to hydrogen cross sections. A fit function of $B(Q)$ world data is newly made and subtracted from cross sections to find values of $A(Q)$. Precision $A(Q)$ structure function data of deuteron are extracted and presented here. Statistical uncertainties are $0.1-1.9 \%$ and systematic uncertainties are $1.2-2.9 \%$. The results of this work is in agreement with the previous Saclay data and are smaller than values from the world's best theoretical calculations. Theoretical calculations need to be reexamined.

Keywords : Deuteron, Cross section, Structure function, A(Q), Electron-deuteron scattering, Jefferson Lab

Student Number : 2002-20398 



\section{Contents}

1 Introduction $\quad 1$

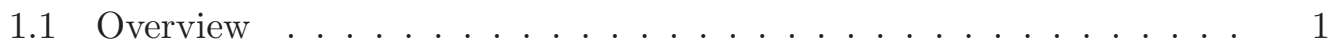

1.2 Elastic Electron - Deuteron Scattering . . . . . . . . . . . 2

1.3 World Data . . . . . . . . . . . . . . . . 6

1.4 Theoretical Calculations . . . . . . . . . . . . . . . . 10

$1.4 .1 \mathrm{EFT} \ldots \ldots \ldots \ldots \ldots \ldots$

1.4.2 Conventional nonrelativistic calculations . . . . . . . . . . 10

1.4.3 Relativistic calculations ............... 13

1.5 Experimental Goals and Kinematic Setting . . . . . . . . . . . 19

2 Experimental Setup $\quad 21$

2.1 Overview of Accelerator . . . . . . . . . . . . . . . . . . 21

2.2 Overview of Hall A . . . . . . . . . . . . . . . . . . 22

2.3 The Detector Package . . . . . . . . . . . . . . . 22

2.3 .1 Triggering . . . . . . . . . . . . . . 25

2.3 .2 Tracking . . . . . . . . . . . . . . 26

2.3 .3 Particle Identification . . . . . . . . . . . . . 26

2.3.4 Collimator ........................ . . 29

2.4 Beam Energy Measurement . . . . . . . . . . . . . . . . . 29

2.5 Beam Position Measurement . . . . . . . . . . . . . 31 
2.6 Beam Current Measurement . . . . . . . . . . . . . . . . . . . . . 31

2.7 Target System . . . . . . . . . . . . . . . . . . . . 32

2.8 Data Acquisition . . . . . . . . . . . . . . . . . 35

3 Data Analysis Part I :

$\begin{array}{ll}\text { Detector Efficiencies and Target Variable Calibrations } & 37\end{array}$

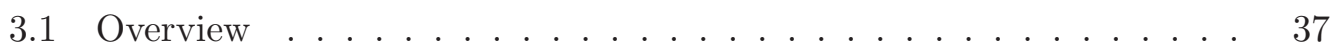

3.2 Event Selection . . . . . . . . . . . . . . . . . . . . . 39

3.2 .1 Event reconstruction . . . . . . . . . . . . . . . . 40

3.2.2 Beam selection cut 1 : BCM cut . . . . . . . . . 40

3.2.3 Beam selection cut 2 : BPM cut . . . . . . . . . . 42

3.2 .4 Cherenkov sum cut . . . . . . . . . . . . . . 47

3.2 .5 Good event cut . . . . . . . . . . . . . . . 47

3.3 Detector Efficiency . . . . . . . . . . . . . . . . . . 52

3.3 .1 Trigger efficiency . . . . . . . . . . . . . . 53

3.3 .2 Tracking efficiency . . . . . . . . . . . . . 54

3.3 .3 Dead time . . . . . . . . . . . . . . 56

3.4 Coordinate System . . . . . . . . . . . . . . . . . . 59

3.4.1 Laboratory coordinate system . . . . . . . . . . . . . . . 59

3.4.2 Target coordinate system . . . . . . . . . . . . . . . 59

3.4.3 Transport coordinate system . . . . . . . . . . . . . 61

3.5 Calibrations of target variables . . . . . . . . . . . . . . 61

3.5.1 Variables of beam and scattered electrons . . . . . . . . 61

3.5.2 Change of target variables . . . . . . . . . . . . 63

3.5 .3 HRS mispointing . . . . . . . . . . . . . . . . . 64

3.5.4 Raster calibration and extended target . . . . . . . . . . 65

4 Data Analysis Part II :

$\begin{array}{ll}\text { Normalization and Yield Extraction } & 71\end{array}$ 
4.1 Beam Current Calibration . . . . . . . . . . . . . . . . . . 71

4.1 .1 Low beam current . . . . . . . . . . . . . . 71

4.1.2 Beam current calibration with silver calorimeter . . . . . 73

4.2 Target Density Correction . . . . . . . . . . . . . . . . 75

4.2 .1 Overview ......................... 75

4.2.2 Yield change by beam position . . . . . . . . . 78

4.2 .3 Target density correction . . . . . . . . . . . . . 81

4.3 Target Length Correction . . . . . . . . . . . . . . . . . . . 86

4.4 Multi-track Events in High Trigger Rate Runs . . . . . . . . . . . . . 86

4.4 .1 Overview .......................... 86

4.4.2 Shower detector energy . . . . . . . . . . . . . . 89

4.4.3 Tracking efficiency with multi-track . . . . . . . . . 92

4.5 Elastic Peak . . . . . . . . . . . . . . . . . . 94

$4.5 .1 \quad W$ spectrum $\ldots \ldots \ldots \ldots \ldots \ldots \ldots$

4.5.2 End cap subtraction in $W$ spectrum . . . . . . . . . . . . 97

4.5.3 Fitting for elastic peak . . . . . . . . . . . . . . 107

4.5.4 Region of elastic peak . . . . . . . . . . . . . . . . 108

4.5.5 End cap subtraction in $Q^{2}$ spectrum . . . . . . . . . . . 116

4.6 Monte Calro Simulation . . . . . . . . . . . . . . . . . . . . . 118

4.6 .1 MCEEP . . . . . . . . . . . . . . . . . 118

4.6.2 Radiation in MCEEP . . . . . . . . . . . . . . . . 121

4.7 Radiative Corrections . . . . . . . . . . . . . . . . 123

4.8 Systematic Uncertainties . . . . . . . . . . . . . . . . . . . 127

5 Cross sections of Carbon and Hydrogen 129

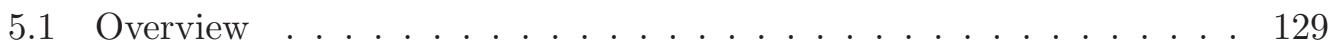

5.2 Acceptance . . . . . . . . . . . . . . . . . 130

5.3 Yield in $Q^{2}$ Spectra . . . . . . . . . . . . . . . . . 131

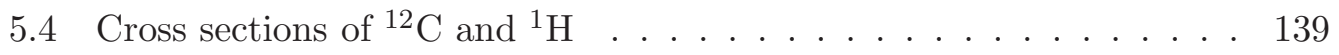


5.5 Systematic Offset Correction :

Cross section Ratio . . . . . . . . . . . . . . . . . . . . . . . . 142

6 Results 145

6.1 Cross section of Deuteron . . . . . . . . . . . . . . . 145

$6.2 \mathrm{~B}(\mathrm{Q})$ Fit Function $\ldots \ldots \ldots \ldots . \ldots \ldots$

$6.3 \mathrm{~A}(\mathrm{Q}) \mathrm{Data} \ldots \ldots \ldots \ldots \ldots \ldots . \ldots \ldots$

6.4 Comparison with World Data and Theoretical Calculations . . . . . 156

$\begin{array}{llr}7 & \text { Conclusions } & 159\end{array}$ 


\section{List of Figures}

1.1 One photon exchange interaction with point charge. . . . . . . . 4

1.2 One photon exchange in electron deuteron interaction. . . . . . . . 4

1.3 Deuteron structure functions. [4] Experimental data are compared with theoretical calculations. . . . . . . . . . . 7

$1.4 \mathrm{~A}(\mathrm{Q})$ world data(ratio to a fit function). [4] Red symbols are from JLab data. . . . . . . . . . . . . . . . . . . . 8

1.5 $\mathrm{A}(\mathrm{Q})$ at low momentum transfer region. Discrepancy is shown among data of Saclay, Mainz and Orsay. . . . . . . . . . . 9

$1.6 \chi \mathrm{PT}$ calculation vs. data.

It is shown as green dashed line. All symbols are the same with Fig.

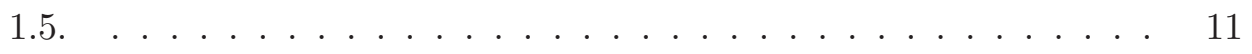

1.7 Yukawa's theory : Nuclear force by OPEP . . . . . . . . . . . . . 12

1.8 Deuteron nuclear force potential . . . . . . . . . . . . . . . . . 12

1.9 Data for $A$ at low and moderate $Q$. [4] They are divided by a fit function and are compared with five nonrelativistic calculations. . . 13

1.10 Diagrams of meson exchange current interaction. . . . . . . . . . 14

1.11 Deuteron structure functions. Experimental data are compared with theoretical calculations. . . . . . . . . . . . . . . 16

1.12 Deuteron structure functions. Experimental data are compared with theoretical calculations. . . . . . . . . . . . . . . . 16

vii 
1.13 Deuteron structure functions. Experimental data are compared with theoretical calculations. . . . . . . . . . . . . . 16

1.14 The data for $A(Q)$, compared to four relativistic calculations. Top panel shows the behavior across the whole range and bottom panel is focused on low energy region.

CIA (red solid line) and RIA (blue long dashed line) of the propagator method and one body + two body currents (black solid line) and one body currents (purple short dashed line) of the hamiltonian method are drawn together. . . . . . . . . . . . . . . . . . . 18

2.1 Lay-out of CEBAF in JLab. [36]

The electron beam is produced at the injector and then accelerated through the two linear accelerators. Two linear accelerators are connected by several recycle rings. . . . . . . . . . . . . . . .

2.2 Lay-out of Hall A. [37]

The electron beam enters through the beamline and interacts with the target. There are two spectrometers in Hall A and each of them can detect electron or hadron. . . . . . . . . . . . . . . . 23

2.3 Sideview of the detector stack. [36] . . . . . . . . . . . . . 24

2.4 Lay-out of a pair of Vertical Drift Chambers. [36] . . . . . . . . . . . 27

2.5 A typical cluster of hits in a VDC plane. [36] The HV planes are held at a potential of approximately $-4 \mathrm{kV}$, while the wires are effectively grounded. . . . . . . . . . . . . . . .

2.6 Schematic lay-out of part of the shower detectors in HRS-L (top) and HRS-R (bottom). [36] Particles enter from the bottom of the figure. 28

2.7 Draft of the left arm collimator. This has small 2 msr area. . . . . . 30

2.8 Draft of silver calorimeter. Beam goes to the slug and changes the temperature of the slug. The beam current can be calculated from the temperature change. $\ldots \ldots \ldots \ldots$ 
2.9 Hall A targets. [37] The top panel shows liquid targets and the bottom panel shows solid targets. . . . . . . . . . . . . . . 34

3.1 Lay-out of data analysis . . . . . . . . . . . . . . . . . . 39

3.2 Charge accumulation vs. time. Data points from ROC10 are drawn in top panel and ones from scaler are in bottom panel. Because ROC10 is much faster than scaler, there are much more points in top panel.

3.3 Beam current vs. time from ROC11 data. Zero current points in top panel are removed in bottom panel after the BCM cut is applied. . . 44

3.4 Time vs. event id from ROC11 data. After the beam is turned on back, the first event is placed at the initial time of a ROC11 period. Time values are discrete because ROC11 data are recorded at each

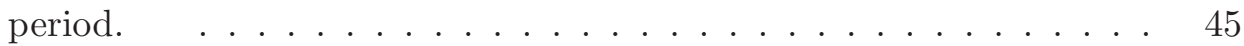

3.5 Contour plot of BPM x.

The $x$ axis is event id and the $y$ axis is $x_{\text {beam }}$. Beam positions of a unstable run are not uniform like the plot in bottom panel. . . . . . 46

3.6 Distributions of $W$ and $Q^{2}$ from two different $x_{\text {beam }}$ regions in a run. The distribution of $Q^{2}$ shows maximum $2 \%$ difference and it is closely related with cross section. . . . . . . . . . . . . 48

$3.7 x_{\text {beam }}$ becomes uniform distribution after BPM cut is applied. . . . 49

3.8 A distribution of Cherenkov sum. The vertical line is the criteria(50) for the Cherenkov sum cut. . . . . . . . . . . . . . 50

3.9 The $W$ distribution from a run with maximum $\pi^{-}$background. Black line : before $\pi^{-}$is removed. Red line : before $\pi^{-}$is removed. Totally $2.5 \%$ of events are removed. . . . . . . . . . . . . 50

3.10 Sieve cut in the $x$ axis and $y$ axis in Transport coordinate system. Two pairs of vertical red lines define the cuts. . . . . . . . . . 51

3.11 Momentum acceptance cut in a white spectrum. Two vertical red lines define the cut. . . . . . . . . . . . . . . . 52 
3.12 Momentum distributions from a cosmic run. The bottom plot is made from the top plot after the good track cut is applied. Most cosmic events are removed by tracking cut. . . . . . . . . . . . .

3.13 Distributions of number of tracks. The top panel is from a high rate $\operatorname{run}(128 \mathrm{kHz})$ and the bottom panel is from an ordinary run $(0.3 \mathrm{kHz})$. Multi-track events occupy $15 \%$ of total events in top panel. . . . . . 57

3.14 The Laboratory coordinate system is seen from the sky. . . . . . . 60

3.15 Target coordinate system(blue) and Transport coordinate system at the detector entrance(red). . . . . . . . . . . . . . . 61

3.16 Target variables . . . . . . . . . . . . . . . . 63

3.17 The change of variables $\left(z_{\text {react }}\right.$ and beam position $\left.\mathrm{x}\right)$ after calibration. Black line : before calibration ; Red line : after calibration . . . . 66

3.18 The change of $d p_{\text {tg }}$ after calibration for ${ }^{12} \mathrm{C}(\mathrm{Top})$ and ${ }^{2} \mathrm{D}$ (Bottom). Black line : before calibration ; Red line : after calibration . . . . 67

4.1 Beam current vs. BCM d1 at calibration runs.

Black symbols are data points from OLO2 current and red symbols are silver calorimeter data. The blue line is an extrapolation of linear fit function made from OLO2 data in the region $I \geq 10 \mu \mathrm{A}$. Whole data points are drawn in top panel and bottom panel is focused on low current region. Silver calorimeter data agree with fit function better than $\mathrm{OLO} 2$ data. . . . . . . . . . . . . . . 76

4.2 Data variation from the new fit function in Eq. 4.1.

The $y$ axis is $\frac{\text { data-fit function }}{\text { fit function }}$. A new data set is chosen from combinations of $\operatorname{OLO} 2$ currents $(I \geq 10 \mu A)$ and silver calorimeter currents $(1 \mu A<$ $I<10 \mu A)$. The fit function agrees with data within $\pm 0.5 \%$ down to

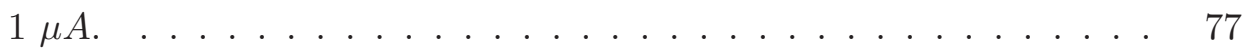


4.3 Contour plot of beam position. The top panel and bottom panel are from two consecutive runs. The average beam position difference between two runs is approximately $3 \mathrm{~mm} . \ldots \ldots . \ldots 79$

4.4 The $Q^{2}$ distribution of two consecutive runs in Fig. 4.3. The black curve is shifted from the red curve and they agree in the center region around $0.074\left[(\mathrm{GeV} / \mathrm{c})^{2}\right]$. Two distributions have similar cross section values but normalized yields are different because of beam position.

4.5 Normalized yield vs. beam current for ${ }^{2} \mathrm{D}$ runs.

Yields are expressed as ratio to a sample run. The top panel yield is without the beam position correction and the bottom panel is with the correction. The total density variation is less than $2 \%$ over $30 \mu A$. 82

4.6 The yield variation from the fit function in percentage.

The RMS deviation is $0.25 \%$ at $5 \mu \mathrm{A} \ldots \ldots . \ldots . \ldots 83$

4.7 Top panel : Normalized yield vs. beam current for ${ }^{1} \mathrm{H}$ runs.

Yield are expressed as ratio to a sample run. Bottom panel : The yield variation from the fit function in percentage. . . . . . . . . . . 84

4.8 Top panel : Normalized yield vs. beam current for ${ }^{12} \mathrm{C}$ runs.

Yield is expressed as ratio to a sample run. Bottom panel : The yield variation from the fit function in percentage. The yield is not correlated with beam current. . . . . . . . . . . . 85

4.9 U1 wire efficiency.

$x$ axis is wire number and $y$ axis is wire efficiency. Top panel is from a $24 \mathrm{kHz}$ run and bottom panel is from a $200 \mathrm{kHz}$ run. . . . . . . . 88

4.10 Preshower energy distribution.

The top panel is made from one track events. The bottom panel is made from multi-track events. The blue curve is the sum of green curve and red curve. There exist two peaks because of two electrons

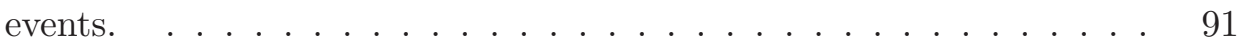


4.11 Top : $W$ distribution of a ${ }^{2} \mathrm{D}$ run at the highest trigger rate.

Bottom : $Q^{2}$ distribution of a ${ }^{2} \mathrm{D}$ run at the highest trigger rate. The black line is before the multi-track correction and red line is after the correction. . . . . . . . . . . . . . . . .

4.12 The increment ratio of $Q^{2}$ distribution after multi-track correction is applied. Ratios are approximately $7 \%$ in the center region(meaningful acceptance region). . . . . . . . . . . . . . . 96

$4.13 W$ distribution of ${ }^{1} \mathrm{H}$.

The elastic peak and radiation tail of ${ }^{1} \mathrm{H}$ are shown. Below elastic peak, there are events from $\mathrm{Al}$. windows and they are convoluted with ${ }^{1} \mathrm{H}$ data. . . . . . . . . . . . . . . . . . . .

$4.14 W$ distribution of ${ }^{2} \mathrm{D}$.

There is an elastic peak and it starts to disintegrate $2.22 \mathrm{MeV}$ apart from the peak. Inelastic events are shown above the peak. Below elastic peak, there are events from $\mathrm{Al}$. windows and they are convoluted with ${ }^{2} \mathrm{D}$ data. . . . . . . . . . . . . . . . . . 98

$4.15 W$ distribution of ${ }^{12} \mathrm{C}$.

${ }^{12} \mathrm{C}$ has many excited states. Especially there are three states within $10 \mathrm{MeV}$ apart from the ground state at $4.44 \mathrm{MeV}, 7.65 \mathrm{MeV}$ and 9.64

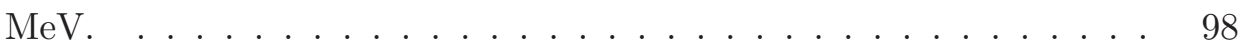

$4.16 W$ distribution of ${ }^{27} \mathrm{Al} \ldots \ldots \ldots . \ldots \ldots 9$

$4.17 W$ distribution of ${ }^{181} \mathrm{Ta}$. . . . . . . . . . . . . . . 99

4.18 Procedure of subtracting $\mathrm{Al}$. windows from liquid target data : The black points are LD2 events before subtraction. The vertical line defines $W_{\mathrm{Al}}$. which separates $\mathrm{Al}$. windows from ${ }^{2} \mathrm{D}$ events. The top panel represents the $W$ distribution of a ${ }^{2} \mathrm{D}$ run. There are two peaks from $\mathrm{Al}$. walls and ${ }^{2} \mathrm{D}$. The bottom panel shows $\mathrm{Al}$. windows distribution after the cut $W<W_{\mathrm{Al}}$ is applied. . . . . . . . . . . . . . . 101 
4.19 Procedure of subtracting Al. windows from liquid target data : The red points are dummy events. The vertical line is the same the value in Fig. 4.18. The top panel shows dummy walls distributions after the cut $W<W_{\mathrm{Al}}$. is applied. The bottom panel is the distribution of normalized dummy walls and it has the same number of events with scaled Al. windows. . . . . . . . . . . . . . . 102

4.20 The result of subtracting Al. windows from liquid taret data: The black and pink points are duplicated. The black one is from LD2 events before subtraction. The pink one is the ${ }^{2} D$ distribution after subtraction. Events from Al. windows are almost removed. . . . . . 103

4.21 The change of $W$ distribution at the highest angle. . . . . . . . . . . 103

4.22 The $\mathrm{W}$ distribution of dummy walls and Al. windows at the lowest angle : Four histogram have prominently different distributions. . . . 106

4.23 The $\mathrm{W}$ distribution of dummy walls and $\mathrm{Al}$. windows at the highest angle : Four histogram have prominently different distributions. . . . 106

4.24 Fit over ${ }^{1} \mathrm{H}$ peak. The top plot is at the lowest angle and bottom plot is at the highest angle. The $x$ axis is $W-M_{\mathrm{tg}}[\mathrm{MeV}]$. Al. walls are already subtracted. . . . . . . . . . . . . . . . 109

4.25 Fit over ${ }^{2} \mathrm{D}$ peak. The top plot is at the lowest angle and bottom plot is at the highest angle. The $x$ axis is $W-M_{\mathrm{tg}}[\mathrm{MeV}]$. Inelastic events are seen above elastic peak. Al. walls are already subtracted. 110

4.26 Fit over ${ }^{12} \mathrm{C}$ peak. The top plot is at the lowest angle and bottom plot is at the highest angle among available runs. The $x$ axis is $W-M_{\mathrm{tg}}$ $[\mathrm{MeV}]$. The first excited state is shown next to elastic peak. . . . . . 111 
4.27 Procedure of elastic peak selection in the $W$ distribution of ${ }^{2} \mathrm{D}$.

Two vertical lines define the elastic peak region.

Black line : data ; Green line : inelastic events ; Red line : elastic data

The distribution of inelastic events are estimated and the contribution is less than $0.1 \%$ of elastic events in the peak region. . . . . . . . 113

4.28 Elastic peak cut at the largest angle.

Black line : data ; Green line : inelastic events ; Red line : elastic data 114

4.29 The elastic peak region selection for ${ }^{1} \mathrm{H}$ target. . . . . . . . . . . . . 115

4.30 The elastic peak region selection for ${ }^{12} \mathrm{C}$ target. Events from the first excited state are excluded. . . . . . . . . . . . . . . . 115

$4.31 Q^{2}$ distributions of two Al. windows and pure ${ }^{2} D$. Their shapes are quite different. . . . . . . . . . . . . . . . . . 117

4.32 Top panel : $Q^{2}$ distributions made by two methods.

Red line : $\omega\left(Q^{2}\right)$; Black line $\omega(W)$

Bottom panel : The ratio distribution of two functions. $\omega(W)$ could give a few $\%$ error. . . . . . . . . . . . . . . . . . 119

4.33 Radiative corrections.

Red line : Experimental data ; Black line : MCEEP simulation.

Top panels are $Q^{2}$ distributions of data(only elastic events) and $Q^{2}$ simulation. Middle panels are $Q^{2}$ simulation without the radiation effect and the efficiency histogram of radiative correction. The bottom panel shows the data distribution after the radiative correction is applied. . . . . . . . . . . . . . . . . . 125 
$4.34 Q^{2}$ distributions of ${ }^{1} H$ (Top panel) and ${ }^{2} D$ (Bottom panel) after the radiative correction is applied.

Black line : Before correction; Red line : After corrected.

The recovery of deuteron is bigger because the elastic peak cut in ${ }^{2} D$ is narrower. . . . . . . . . . . . . . . . . . 126

5.1 Yield of carbon at Kinematics 1.

Left : Yield in $Q^{2}$ spectra. Right : Yield in momentum ratio over the central momentum. . . . . . . . . . . . . . . . . . . . 132

5.2 Yield of carbon at Kinematics 2.

Left : Yield in $Q^{2}$ spectra. Right : Yield in momentum ratio over the central momentum. . . . . . . . . . . . . . . . . . . . 132

5.3 Yield of carbon at Kinematics 4.

Left : Yield in $Q^{2}$ spectra. Right : Yield in momentum ratio over the central momentum. . . . . . . . . . . . . . . . . . . . . 132

5.4 Yield of carbon at Kinematics 7.

Left : Yield in $Q^{2}$ spectra. Right : Yield in momentum ratio over the central momentum. . . . . . . . . . . . . . . . . 133

5.5 Yield of carbon at Kinematics 8.

Left : Yield in $Q^{2}$ spectra. Right : Yield in momentum ratio over the central momentum. . . . . . . . . . . . . . . . . . . . . 133

5.6 Yield of carbon at Kinematics 9.

Left : Yield in $Q^{2}$ spectra. Right : Yield in momentum ratio over the central momentum. . . . . . . . . . . . . . . . . . . . 133

5.7 Yield of carbon at Kinematics 10.

Left : Yield in $Q^{2}$ spectra. Right : Yield in momentum ratio over the central momentum. . . . . . . . . . . . . . . . . . . . . . . 134 
5.8 Yield of carbon at Kinematics 11.

Left : Yield in $Q^{2}$ spectra. Right : Yield in momentum ratio over the central momentum. . . . . . . . . . . . . . . . . . . . . 134

5.9 Yield of hydrogen at Kinematics 1.

Left : Yield in $Q^{2}$ spectra. Right : Yield in momentum ratio over the central momentum. . . . . . . . . . . . . . . . . . . . 135

5.10 Yield of hydrogen at Kinematics 2.

Left : Yield in $Q^{2}$ spectra. Right : Yield in momentum ratio over the central momentum. . . . . . . . . . . . . . . . . . 135

5.11 Yield of hydrogen at Kinematics 3.

Left : Yield in $Q^{2}$ spectra. Right : Yield in momentum ratio over the central momentum. . . . . . . . . . . . . . . . . . 135

5.12 Yield of hydrogen at Kinematics 4.

Left : Yield in $Q^{2}$ spectra. Right : Yield in momentum ratio over the central momentum. . . . . . . . . . . . . . . . . . . . 136

5.13 Yield of hydrogen at Kinematics 5.

Left : Yield in $Q^{2}$ spectra. Right : Yield in momentum ratio over the central momentum. . . . . . . . . . . . . . . . . . . . 136

5.14 Yield of hydrogen at Kinematics 6 .

Left : Yield in $Q^{2}$ spectra. Right : Yield in momentum ratio over the central momentum. . . . . . . . . . . . . . . . . . 136

5.15 Yield of hydrogen at Kinematics 7.

Left : Yield in $Q^{2}$ spectra. Right : Yield in momentum ratio over the central momentum. . . . . . . . . . . . . . . . . . . . 137

5.16 Yield of hydrogen at Kinematics 8.

Left : Yield in $Q^{2}$ spectra. Right : Yield in momentum ratio over the central momentum. . . . . . . . . . . . . . . . . . . . 137 
5.17 Yield of hydrogen at Kinematics 10.

Left : Yield in $Q^{2}$ spectra. Right : Yield in momentum ratio over the central momentum. . . . . . . . . . . . . . . . . . . 137

5.18 Yield of hydrogen at Kinematics 11.

Left : Yield in $Q^{2}$ spectra. Right : Yield in momentum ratio over the central momentum. . . . . . . . . . . . . . 138

5.19 Yield of hydrogen at Kinematics 12.

Left : Yield in $Q^{2}$ spectra. Right : Yield in momentum ratio over the central momentum. . . . . . . . . . . . . . . . . . 138

5.20 Yield of hydrogen at Kinematics 13.

Left : Yield in $Q^{2}$ spectra. Right : Yield in momentum ratio over the central momentum. . . . . . . . . . . . . . . . 138

5.21 Carbon cross section ratio over a world data fit. Data are $96.5 \pm 1.6$ $\%$ over the fit. . . . . . . . . . . . . . . . . . 140

5.22 Hydrogen cross section ratio over a world data fit. Data are $94.3 \pm 1.3$

$\%$ over the fit. . . . . . . . . . . . . . . . . . 141

6.1 Cross sections from Kinematics 1. . . . . . . . . . . . . . . 147

6.2 Cross sections from Kinematics 2. . . . . . . . . . . . . . . . . 147

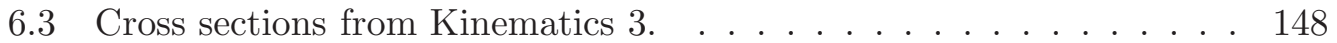

6.4 Cross sections from Kinematics 4. . . . . . . . . . . . . . . . . 148

6.5 Cross sections from Kinematics 5. . . . . . . . . . . . . . . . . 149

6.6 Cross sections from Kinematics 6. . . . . . . . . . . . . . . . 149

6.7 Cross section from Kinematics $7 . \quad \ldots \ldots \ldots$

6.8 Cross sections from Kinematics 8. . . . . . . . . . . . . . . . 150

6.9 Cross sections from Kinematics 10. . . . . . . . . . . . . . . . 151

6.10 Cross sections from Kinematics 11. . . . . . . . . . . . . . . . 151

6.11 Cross sections from Kinematics 12. . . . . . . . . . . . . . . 152

6.12 Cross sections from Kinematics $13 . \quad \ldots \ldots \ldots$ 
$6.13 B(Q) / \mathrm{B}_{\text {fit }}$ vs. Q.

A fit(black line) of 2nd order polynomial is newly applied to update the old fit function $\mathrm{B}_{\mathrm{fit}}$. . . . . . . . . . . . . . . . . . . 154

$6.14 B(Q) /($ New fit function) vs. Q.

The $y$ axis is the ratio of $B(Q)$ data to the modified function. . . . 154

6.15 New data and world data. . . . . . . . . . . . . . . 157

6.16 New data are compared with two relativistic calculations and ChiPT. The blue solid curve is the relativistic calculations from Gross CIA, the red dot curve is the relativistic calculations from Schivilla and the green dashed curve is the ChiPT from Phillips. . . . . . . . . . . . 158 


\section{List of Tables}

1.1 Some measurements of $A(Q) \ldots \ldots \ldots \ldots$

1.2 Kinematic settings. . . . . . . . . . . . . . 20

2.1 Main design characteristics of the Hall A High Resolution Spectrometers. [36] The resolution values are for the FWHM. . . . . . . . . 25

4.1 Beam current at liquid targets . . . . . . . . . . . . . . 73

4.2 Beam current and BCM d1 from calibration runs . . . . . . . . . . 74

4.3 The trigger rate and multi-track ratio at high rate runs . . . . . 87

4.4 Ratio of two electrons events over one electron event . . . . . . . . . 92

4.5 Systematic Errors . . . . . . . . . . . . . . . . . 128

6.1 Values of $A(Q)$ and deuteron cross sections. . . . . . . . . . 155 



\section{Chapter 1}

\section{Introduction}

\subsection{Overview}

Nucleon-nucleon interaction has been one of the most ineresting subjects in nuclear physics. Especially, deuteron is the only nucleus made of two nucleons and a keyissue in understanding the interaction. There have been many efforts to understand properties of deuteron in experimental works and theoretical calculations. Deuteron inner structure can be described by three structure functions known as $A(Q), B(Q)$ and $T_{20}(Q)$. No theoretical model explains three deuteron structure functions well across the whole $Q$ range yet.

Though QCD is the fundamental theory of quarks and their compounds, it is not applicable to a problem with energy scale of a nucleus. Instead, conventional potential models are developed. Several nuclear potentials are introduced to describe the nucleon-nucleon interaction and deuteron is treated as a bound state of two nucleons. They are based on the OBEP(one boson exchange potential) model and are parametrized from the scattering data of neutron-proton and proton-

proton. At present status, several potentials are derived with the reduced $\chi^{2} \approx 1$ for parametrization fitting [1].

There have been several experiments to measure deuteron structure functions. 
Especially $A(Q)$ function, which is dominant in low $Q$ range, shows the best match with theoretical calculations. However discrepancies are still existing among experimental data and they exceed $\sim 10 \%$ in two ranges : $0.2<Q<0.4 \mathrm{GeV} / \mathrm{c}$ and $Q>1.0 \mathrm{GeV} / \mathrm{c}$ and the difference is beyond the experimental uncertainties. Especially in the first region, relativistic effects of deuteron become prominent. So present world data cannot determine even the sign of the leading relativistic corrections, or of the convergence of chiral perturbation calculation.

This experiment is motivated to resolve the discrepancies among $A(Q)$ structure function data in the low momentum transfer region $0.2<Q<0.4 \mathrm{GeV} / \mathrm{c}$. In this chapter, kinematic variables of scattering process are defined and previous experimental world data and theoretical calculations are introduced. Finally, the kinematic setting of this experiment is explained.

\subsection{Elastic Electron - Deuteron Scattering}

The electromagnetic cross section of electron scattering with a point charge is well known from QED as Mott cross section. [2] Fig. 1.1 shows the scattering diagram and the cross section is given as

$$
\left.\frac{\mathrm{d} \sigma}{\mathrm{d} \Omega}\right|_{\mathrm{NS}}=\left(\frac{Z^{2} \alpha^{2} E^{2}}{4 k^{4} \sin ^{4} \frac{\theta}{2}}\right)\left\{1-v^{2} \sin ^{2} \frac{\theta}{2}\right\},
$$

where $k\left(=\left|\mathbf{k}_{\mathbf{i}}\right|=\left|\mathbf{k}_{\mathbf{f}}\right|\right)$ is the electron momentum, $E$ is the electron energy, $v=k / E$, $Z$ is the charge of interacting field and $\theta$ is the scattering angle of electron.

If an electron interacts with charge distribution $Z e \rho(\mathbf{x})$, the cross section is modified by the form factor $F\left(Q^{2}\right)$ as [3]

$$
\frac{\mathrm{d} \sigma}{\mathrm{d} \Omega}=\left.\frac{\mathrm{d} \sigma}{\mathrm{d} \Omega}\right|_{\mathrm{NS}} \times\left|F\left(Q^{2}\right)\right|^{2}, \quad F\left(Q^{2}\right)=F(\mathbf{q})=\int \rho(\mathbf{x}) e^{i \mathbf{q} \cdot \mathbf{x}} \mathrm{d}^{3} x
$$

Deuteron also has internal structure of quarks and gluons. The structure can be 
examined by elastic electromagnetic scattering with electron beam. Fig. 1.2 shows the Feynman diagram of the leading order. The electron momenta are expressed by $k, k^{\prime}$ and the nucleus momenta are expressed by $p, p^{\prime}$ in the figure. The 4-momentum transfer between the two particles is expressed by $Q^{2}$ and the status of the scattered nucleus can be described by invariant mass $W$. They are defined as

$$
\begin{aligned}
& Q^{2}=-q^{2}=-\left(k-k^{\prime}\right)^{2} \\
& W^{2}=(P+q)^{2}=M^{2}+2 M \nu+q^{2} \\
& W=\sqrt{W^{2}}
\end{aligned}
$$

where $\mathrm{P}$ is the 4-momentum of nucleus and $\nu=E-E^{\prime}$. The deuteron elastic cross section can be extracted from the scattering amplitude of interaction of two currents: one leptonic and the other, hadronic. If deuteron is structureless particle, the cross section would have the same form with that of electron-muon scattering. However deuteron is a composite of quarks and gluons and has complex internal structure. Deuteron current can be written by general current form satisfying Lorentz invariance. Several more constraints such as current conservation and invariance of parity and time-reversal can be applied to the current equation. Then only three terms remains and the current matrix form of deuteron is given as $[4,5]$

$$
\begin{aligned}
G_{\lambda_{d}^{\prime} \lambda d}^{\mu}\left(P^{\prime}, P\right)=-\{ & G_{1}\left(Q^{2}\right)\left(\xi_{\lambda_{d}^{\prime}}^{*}\left(P^{\prime}\right) \cdot \xi_{\lambda_{d}}(P)\right)\left(P^{\prime}+P\right)^{\mu} \\
& +G_{2}\left(Q^{2}\right)\left[\xi_{\lambda_{d}}^{\mu}(P)\left(\xi_{\lambda_{d}^{\prime}}^{*}\left(P^{\prime}\right) \cdot q\right)-\xi_{\lambda_{d}^{\prime}}^{\mu *}\left(P^{\prime}\right)\left(\xi_{\lambda_{d}}(P) \cdot q\right)\right] \\
& \left.\quad-G_{3}\left(Q^{2}\right) \frac{1}{2 M_{d}^{2}}\left(\xi_{\lambda_{d}^{\prime}}^{*}\left(P^{\prime}\right) \cdot q\right)\left(\xi_{\lambda_{d}}(P) \cdot q\right)\left(P^{\prime}+P\right)^{\mu}\right\}
\end{aligned}
$$

where $M_{d}$ is the deuteron mass, $P$ and $P^{\prime}$ are the initial and final deuteron four momenta, $\xi_{\lambda_{d}^{\prime}}^{\mu *}\left(P^{\prime}\right)$ are the polarization four vectors for the initial and final deuteron states.

Three $G_{i}$ s can be rearranged as the electric monopole, electric quadrupole, mag- 


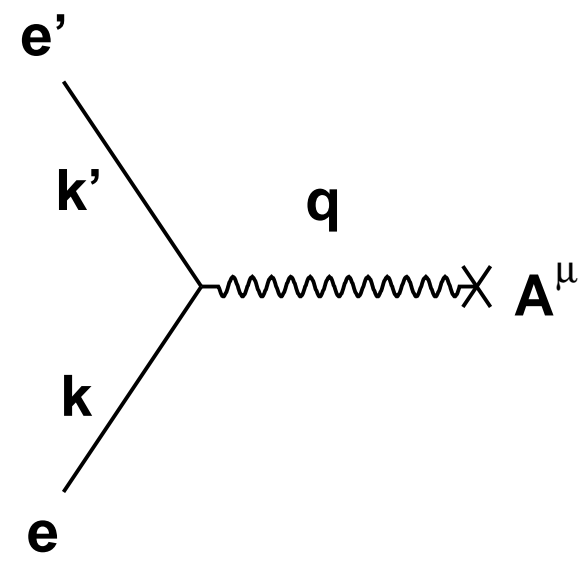

Figure 1.1: One photon exchange interaction with point charge.

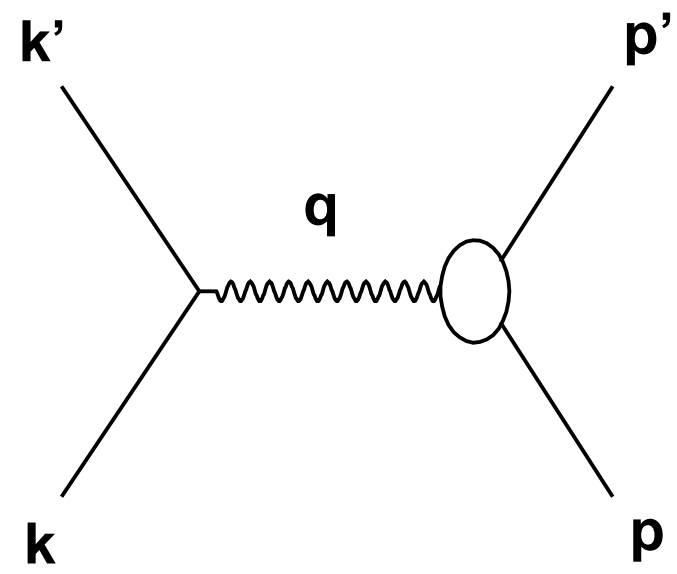

Figure 1.2: One photon exchange in electron deuteron interaction. 
netic dipole term of $G_{c}, G_{M}$ and $G_{Q}$.

$$
\begin{aligned}
& G_{C}\left(Q^{2}\right)=G_{1}\left(Q^{2}\right)+\frac{2}{3} \eta G_{Q}\left(Q^{2}\right) \\
& G_{M}\left(Q^{2}\right)=G_{2}\left(Q^{2}\right) \\
& G_{Q}\left(Q^{2}\right)=G_{1}\left(Q^{2}\right)-G_{2}\left(Q^{2}\right)+(1+\eta) G_{3}\left(Q^{2}\right),
\end{aligned}
$$

where $\eta=\frac{Q^{2}}{4 M_{d}^{2}}$.

The cross section can be expressed in terms of the previous three form factors,

$$
\begin{aligned}
\frac{\mathrm{d} \sigma}{\mathrm{d} \Omega}= & \frac{\sigma_{M}}{1+\left(2 E / M_{d}\right) \sin ^{2}(\theta / 2)} \times\left[A\left(Q^{2}\right)+B\left(Q^{2}\right) \tan ^{2} \frac{\theta}{2}\right] \\
& \times\left[1+\rho_{20} \cdot t_{20}\left(Q^{2}, \theta\right)+2 \operatorname{Re} \rho_{21} \cdot t_{21}\left(Q^{2}, \theta\right)+2 \operatorname{Re} \rho_{22} \cdot t_{22}\left(Q_{2}, \theta\right)\right. \\
& \left.\quad+h \rho_{10} \cdot t_{10}\left(Q^{2}, \theta\right)+2 h \operatorname{Re} \rho_{11} \cdot t_{11}\left(Q^{2}, \theta\right)\right]
\end{aligned}
$$

where $\sigma_{M}$ is the Mott cross section and $A\left(Q^{2}\right), B\left(Q^{2}\right)$ and $t_{i j}$ are combinations of three form factors and known as structure functions.

Terms including $t_{i j}$ functions are related with polarization and only $A\left(Q^{2}\right)$ and $B\left(Q^{2}\right)$ survive in the unpolarized cross section. Therefore the cross section with unpolarized beam and target can be expressed with only two terms of $A\left(Q^{2}\right)$ and $B\left(Q^{2}\right)$ as

$$
\begin{aligned}
& \frac{\mathrm{d} \sigma}{\mathrm{d} \Omega}=\left.\frac{\mathrm{d} \sigma}{\mathrm{d} \Omega}\right|_{\mathrm{NS}} \times\left[A\left(Q^{2}\right)+B\left(Q^{2}\right) \tan ^{2} \frac{\theta}{2}\right] \\
& A\left(Q^{2}\right)=G_{C}^{2}\left(Q^{2}\right)+\frac{2}{3} \eta G_{M}^{2}\left(Q^{2}\right) \frac{8}{9} \eta^{2} G_{Q}^{2}\left(Q^{2}\right) \\
& B\left(Q^{2}\right)=\frac{4}{3} \eta(1+\eta) G_{M}^{2}\left(Q^{2}\right) .
\end{aligned}
$$

$A\left(Q^{2}\right)$ and $B\left(Q^{2}\right)$ are expanded in terms of $\eta$ as zeroth and first order. In the kinematic region of this experiment, $\eta$ is less than 0.03. In addition, $B\left(Q^{2}\right)$ is coupled with $\tan ^{2} \frac{\theta}{2}$ and $\theta$ is less than $70^{\circ}$ during this experiment. Hence $A\left(Q^{2}\right)$ is the dominant term of cross section in the kinematic region of this experiment. 


\subsection{World Data}

World data of deuteron structure functions of $A\left(Q^{2}\right), B\left(Q^{2}\right)$ and $T_{20}\left(Q^{2}\right)$ are drawn in Fig. 1.3 together with several theoretical calculations. Symbols correspond to experimental data and curves correspond to theoretical calculations. Data of JLab Hall A and Hall $\mathrm{C}$ are shown with red symbols. The $x$ axis is $Q^{2}$ in $(\mathrm{GeV} / \mathrm{c})^{2}$ and the $y$ axis is the structure function. Functions $A\left(Q^{2}\right)$ and $B\left(Q^{2}\right)$ vary sensitively over $Q^{2}$ so the $y$ axis is drawn in log scale. It is seen that $A\left(Q^{2}\right)$ is best understood among all three at present status. Especially, black solid curve [7] in $A(Q)$ plot shows the best match among calculations but it still disagrees with $A\left(Q^{2}\right)$ by $20-30 \%$ at $Q^{2}$ of $2-3$ $(\mathrm{GeV} / \mathrm{c})^{2}$. Experimental data of $A(Q)$ are overlapped and differences among them are hidden by log scale. Fig. 1.4 shows world data of $A(Q)$ focused on low $Q$ region. A fit function $A_{\text {fit }}[4]$ is introduced to show differences among data. The $y$ axis is drawn as a ratio of $A(Q)$ to the fit function, $\left(A(Q) / A_{\text {fit }}(Q)\right)$. Usually, $Q^{2}$ and $A\left(Q^{2}\right)$ are conventionally used but they are expressed as a function of $Q$ here to emphasize low energy region. Data of JLab Hall A and Hall C are shown with red symbols. Differences among data sets are shown in two regions around $0.3 \mathrm{GeV} / \mathrm{c}$ and above 1 $\mathrm{GeV} / \mathrm{c}$. Especially, relativistic effects of deuteron start becoming prominent around $0.3 \mathrm{GeV} / \mathrm{c}$. So getting accurate data around $0.3 \mathrm{GeV} / \mathrm{c}$ is essential to find improved theoretical calculation across the whole region because calculations are relatively inaccurate at high $Q$ region where relativistic effects are dominant.

Fig. 1.5 shows experimental data of $A(Q)$ at the problematic region around $\mathrm{Q} \sim$ $0.3 \mathrm{GeV} / \mathrm{c}$. Each data set is summarized in Table. 1.1. Yellow symbols are Saclay data and blue symbols are Mainz data and green symbols are Orsay data. They have high precision systematic errors less than $2 \%$ so the discrepancy should be resolved by new experiment. The Mainz experiment [9] used liquid and gas targets to determine elastic cross sections at 8 beam energies, from 80 to $298.9 \mathrm{MeV}$, with laboratory electron angles from $30^{\circ}$ to $157^{\circ}$. The claimed deuteron cross section systematic uncertainty was $\approx 0.7 \%$ and it is obtained with ratio to hydrogen data. 

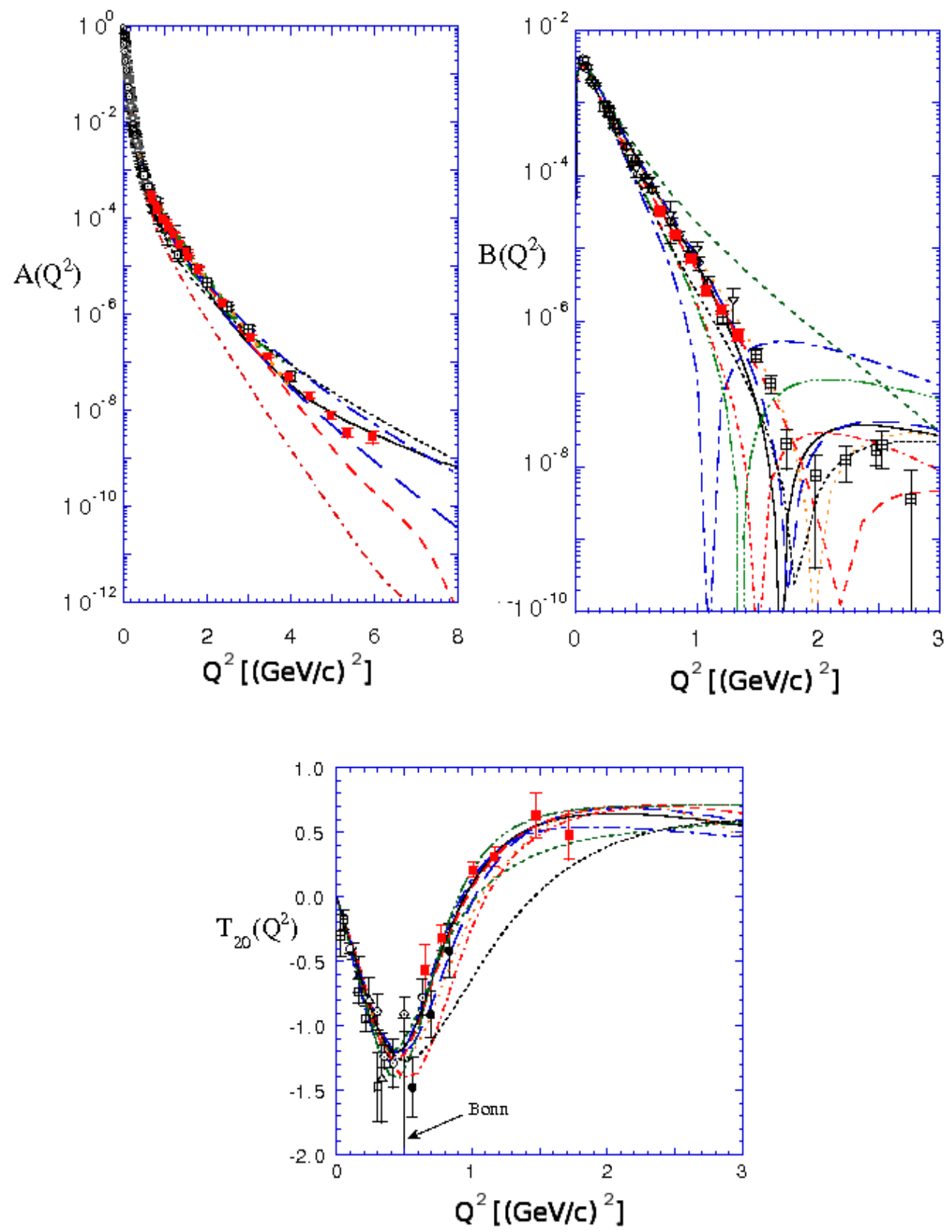

Figure 1.3: Deuteron structure functions. [4] Experimental data are compared with theoretical calculations. 


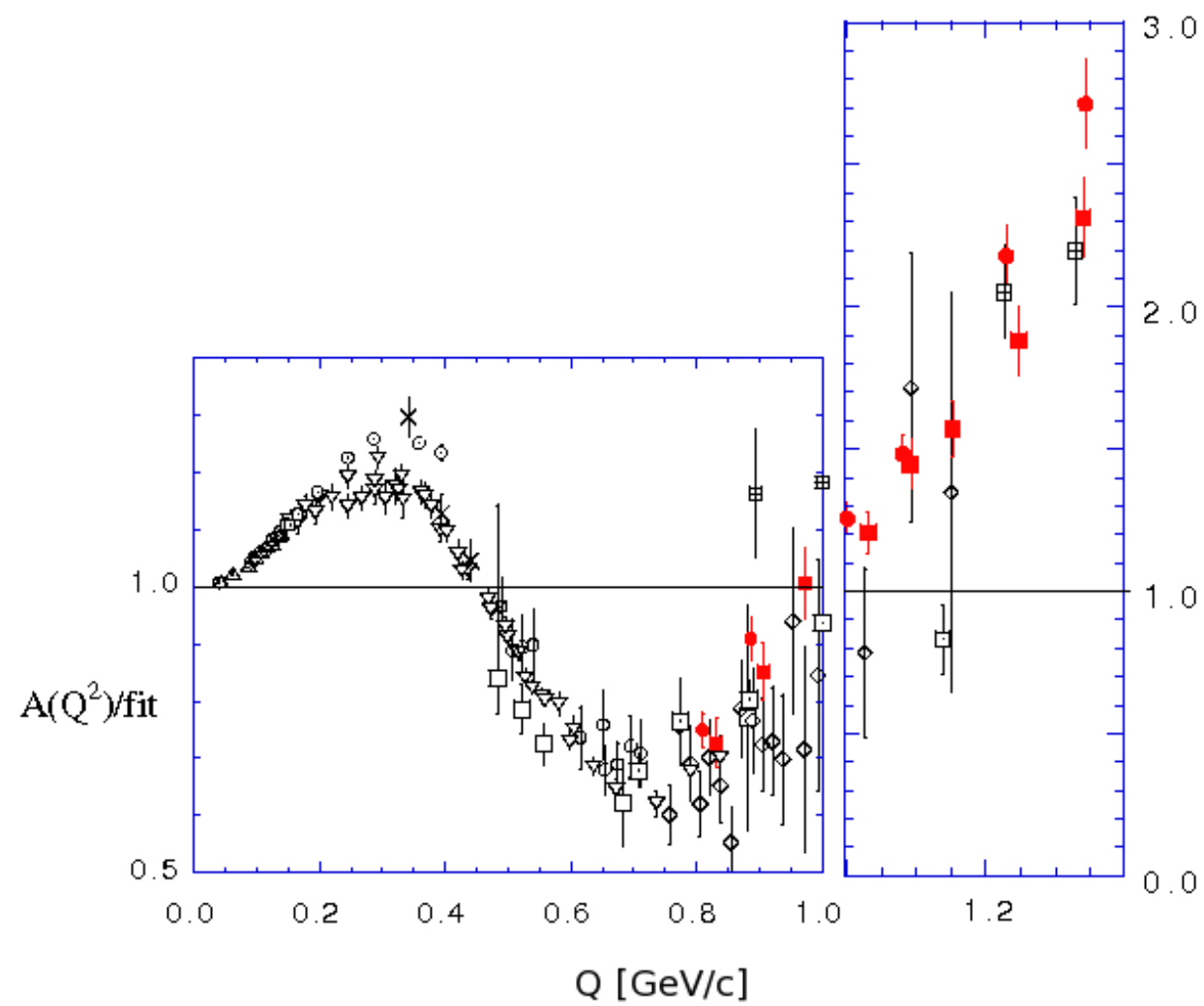

Figure 1.4: $\mathrm{A}(\mathrm{Q})$ world data(ratio to a fit function). [4] Red symbols are from JLab data.

$B(Q)$ is extracted by Rosenbluth separation method. The Saclay data [10] used 4 energies from 200 to $650 \mathrm{MeV}$, with scattering angles from $35^{\circ}$ to $100^{\circ}$. Deuteron cross section is measured directly. The contribution of $B(Q)$ to the cross section was calculated based on previous world data and subtracted. The claimed systematic uncertainties were $1-1.5 \%$. The larger amount of Saclay data, as well as the overlap with higher $Q$ experiments from other labs, suggests that the correct trend is Saclay data. 
Table 1.1: Some measurements of $A(Q)$.

Experiment $Q(\mathrm{GeV} / \mathrm{c})$ Symbol Year and

\begin{tabular}{llll} 
& & & Reference \\
\hline Monterey & $0.04-0.14$ & $\square$ & $1973[8]$ \\
Mainz & $0.04-0.39$ & $\bigcirc$ & $1981[9]$ \\
Saclay ALS & $0.13-0.84$ & & $1990[10]$ \\
Orsay & $0.34-0.48$ & $\triangle$ & $1966[11]$ \\
Stanford & $0.48-0.88$ & $\square$ & $1965[12]$ \\
DESY & $0.49-0.71$ & $\diamond$ & $1971[13]$ \\
CEA & $0.76-1.15$ & $\times$ & $1969[14]$ \\
JLab Hall C & $0.81-1.34$ & $\star$ & $1999[15]$ \\
JLab Hall A & $0.83-2.44$ & $\square$ & $1999[16]$ \\
SLAC E101 & $0.89-2.00$ & + & $1975[17]$ \\
\hline
\end{tabular}

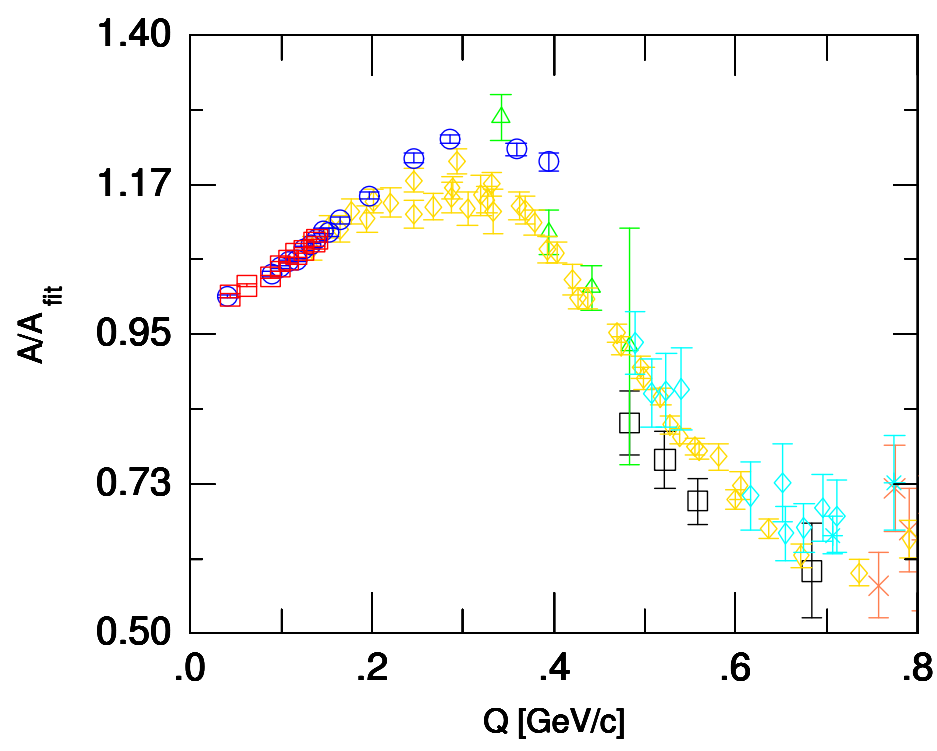

Figure 1.5: $\mathrm{A}(\mathrm{Q})$ at low momentum transfer region. Discrepancy is shown among data of Saclay, Mainz and Orsay. 


\subsection{Theoretical Calculations}

There have been two separate methods describing deuteron structure functions. One is potential models introducing nucleon-nucleon potentials based on OBEP. Another is effective field theory models such as pionless EFT or $\chi$ perturbation theory (ChiPT) which are low energy limit of QCD.[5, 6, 4]

\subsubsection{EFT}

Pionless model describes structure functions well in very low energy region. It is essentially limited below $2 m_{\pi}$ (in Breit frame) because it does not include pion contributions. The range is too low to be applied for this experiment. ChiPT includes pion interaction and it is extended to $\sim 700 \mathrm{MeV}$ at present. Fig. 1.6 compares a recent ChiPt calculation[18] with $A(Q)$ data. The calculation used $\chi \mathrm{PT}$ wave functions for the deuteron with the $\chi \mathrm{PT}$ current operator at NNLO. It is shown as green dotted line and larger than Saclay data and smaller than Mainz data. But this is limited in high momentum transfer region and cannot give consistent explanation across the whole range of $Q^{2}$.

\subsubsection{Conventional nonrelativistic calculations}

Nucleon-nucleon interaction was first explained with meson exchange model by Yukawa as in Fig. 1.7. Later the meson has been identified as pion. In shorter range, heavier bosons such as $\sigma, \rho, \omega$ and $\eta$ contribute to the interaction. Hence the interaction is constructed based on OBEP potential. The potential consists of several terms and form of each term is varied by boson types such as pseudo-scalar, vector and pseudo-vector. There are several potential models for nucleon-nucleon interaction. Whereas all models use the same OPEP potential, there exists differences in handling short range terms.

Conventional nonrelativistic calculations are distinguished by the potential models 


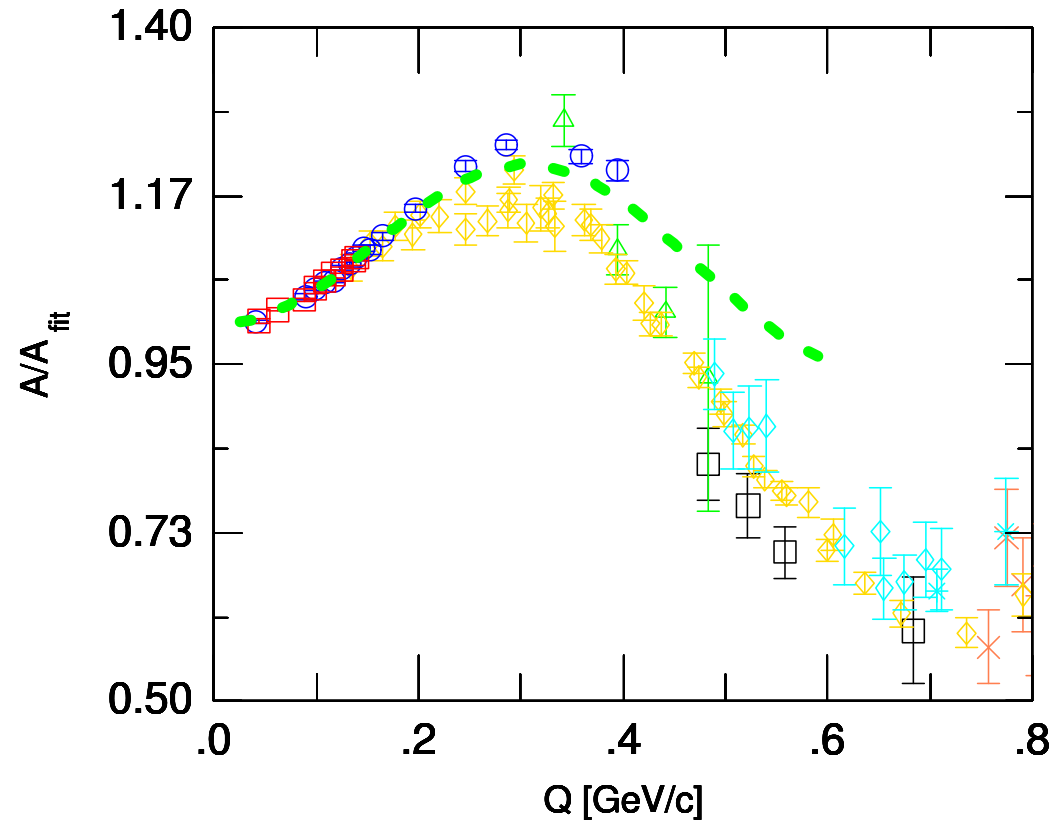

Figure 1.6: $\chi \mathrm{PT}$ calculation vs. data.

It is shown as green dashed line. All symbols are the same with Fig. 1.5.

used.[19, 20, 21] Fig. 1.9 shows several models with different potentials.

- W16 (long dot-dashed)

- CD Bonn (short dashed)

- AV18 (solid)

- IIB (short dot-dashed)

- Paris (long dashed)

The variations among these models are only about $\pm 2 \%$ in the region of our interest. The models lie between the data of Saclay and Mainz in favor of Mainz data. In the high $Q$ region, the variation becomes large because the short range interaction 


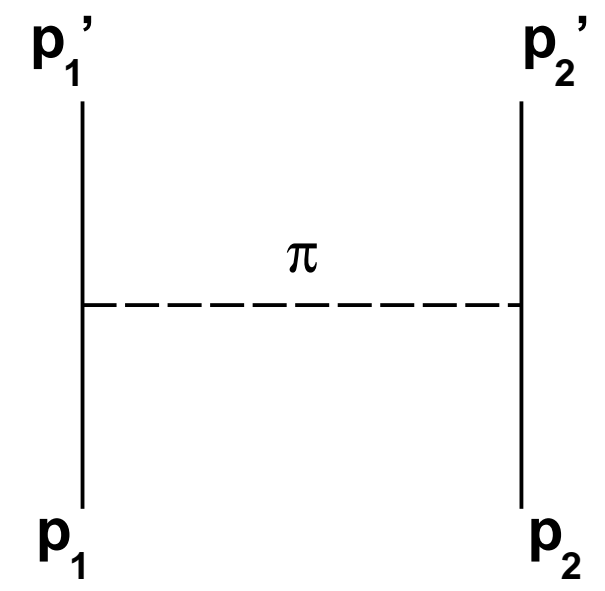

Figure 1.7: Yukawa's theory : Nuclear force by OPEP

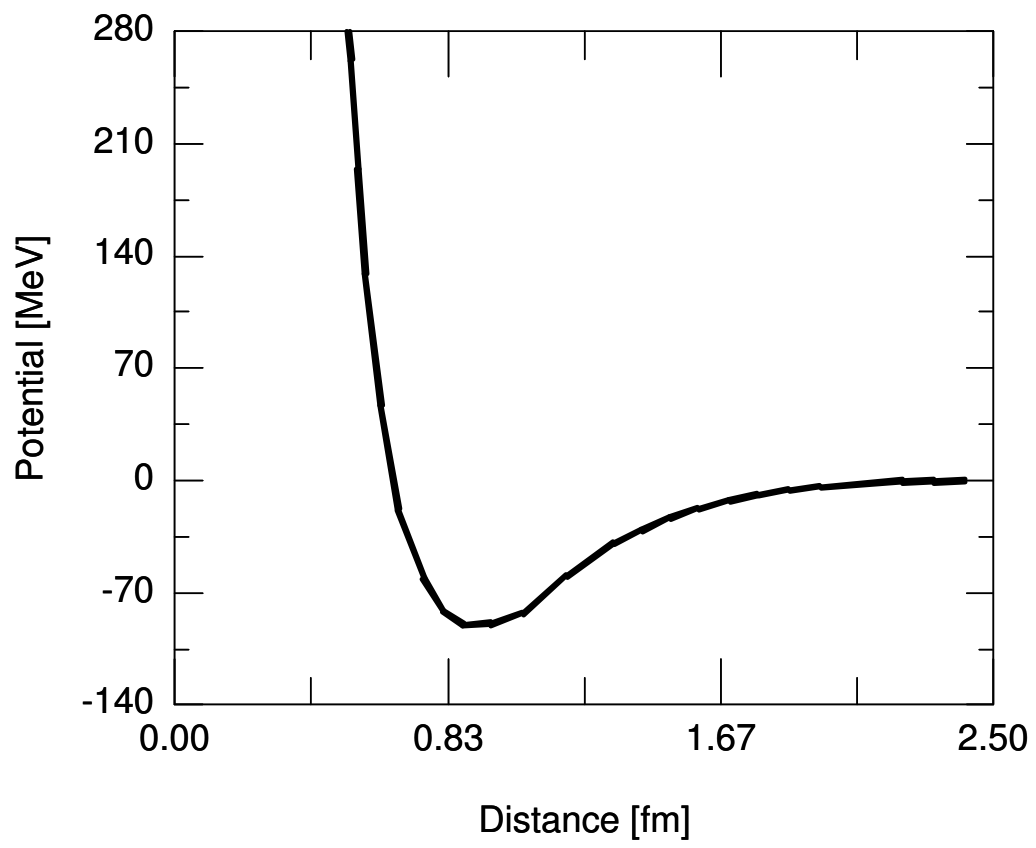

Figure 1.8: Deuteron nuclear force potential 


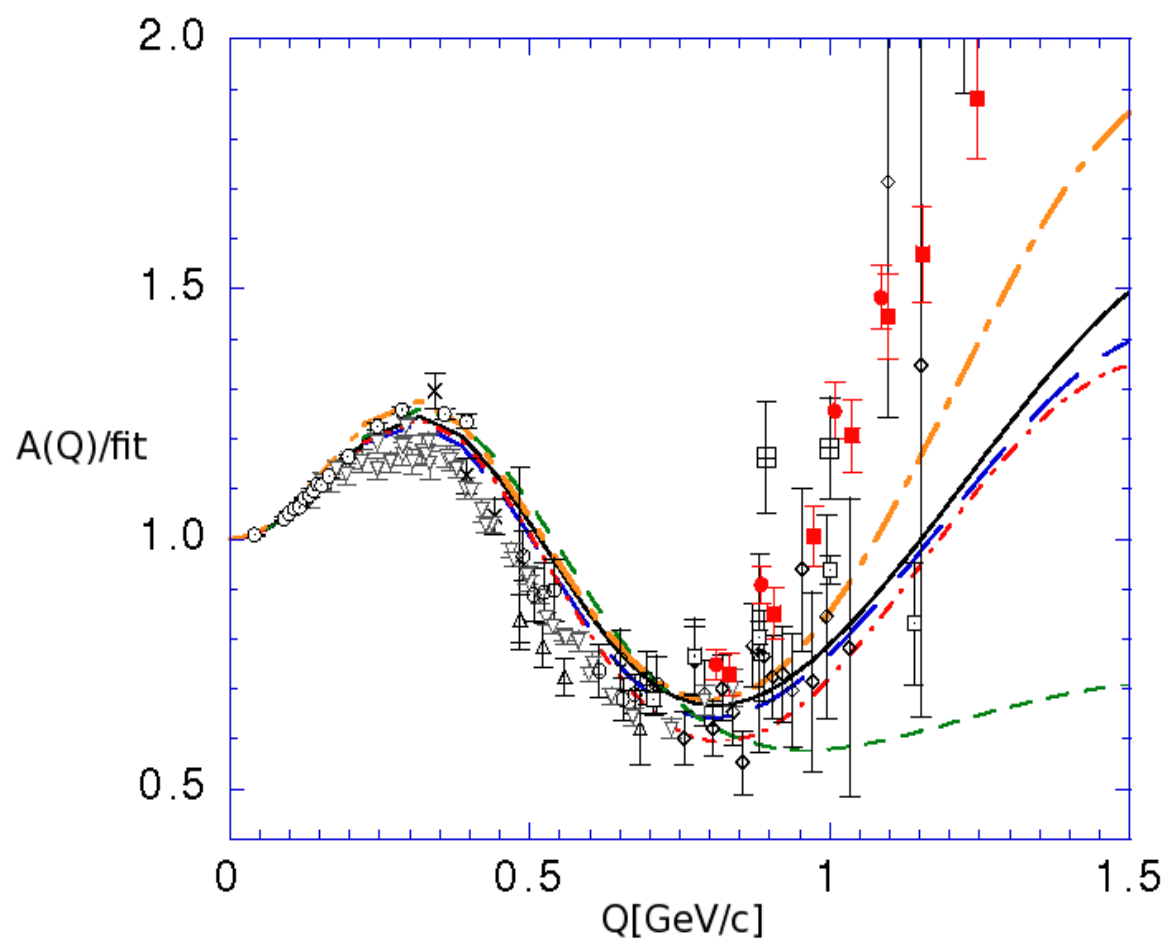

Figure 1.9: Data for $A$ at low and moderate $Q$. [4] They are divided by a fit function and are compared with five nonrelativistic calculations.

is treated differently in each model. Curves are smaller than data points and it indicates that relativistic effects should be included.

\subsubsection{Relativistic calculations}

Considering the fact that the nucleon mass is approximately $1 \mathrm{GeV} / \mathrm{c}^{2}$, relativistic effects should be included in high $Q$ range. There are two types of relativistic models. One is known as hamiltonian dynamics method and another is known as propagator dynamics method. 


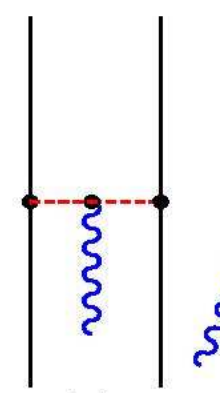

(a)

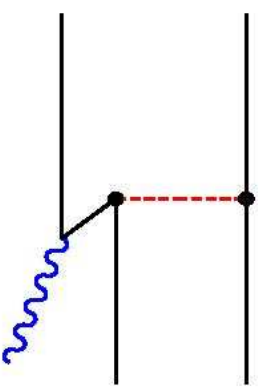

(b)

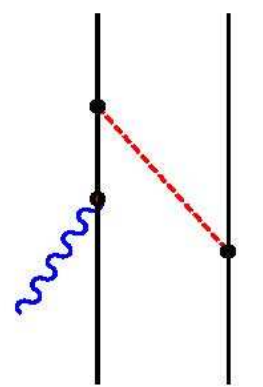

(c)

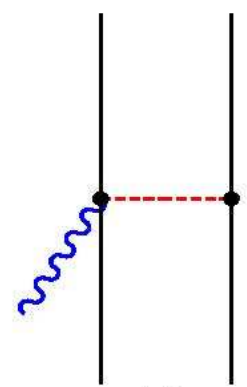

(d)

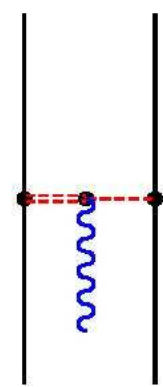

(e)

Figure 1.10: Diagrams of meson exchange current interaction.

\section{Hamiltonian dynamics}

This is based on quantum mechanics. The equation of non-relativistic model is modified to satisfy the Poincare covariance. Methods are divided into three cases of instant form, front form and point form by the difference of quantization method. Details can be found in references. [22, 23, 24, 26, 29, 25]

Hamiltonian in the rest frame of deuteron is defined as

$$
H=2 \sqrt{M^{2}+P^{2}}+v,
$$

where $v$ is combination of parametrized potential and OBEP.

The potential is related with kinematic variables of exchange bosons and nucleons. In addition, the deuteron wave function is obtained after the boost transformation is applied to the rest frame wave function. Whereas non-relativistic approximations are applied in non-relativistic models, calculating equations are treated relativistically in relativistic models. Because of the square root in hamiltonian, negative energy states are excluded and it is assumed that the contribution is negligible. After the deuteron wave function is obtained, the structure functions can be calculated from the scattering matrix elements of interacting currents. If we assume that a interact- 
ing nucleon is regardless of a non-interacting nucleon(impulse approximation), the interaction current operator can be written as

$$
j_{1 \text {-body }}^{\sigma}(\mathbf{q})=\sum_{i=1,2} \frac{1}{2} \overline{u_{i}^{\prime}}\left[F_{1}^{s}(q) \gamma^{\sigma}+\mathbf{i} \frac{F_{2}^{s}(q)}{2 m} \sigma^{\sigma \tau} q_{\tau}\right] u_{i}
$$

where $F_{1}$ and $F_{2}$ are nucleon form factors.

For more accurate calculation, two-body currents(meson exchange currents) need to be considered. Possible Feynman diagrams are shown in Fig. 1.10. These diagrams become important in high $Q$ region.

\section{Propagator dynamics}

Another method is called as propagator dynamics method. [27, 28] It is a field theoretical method based on the Bethe-Salpeter equation and it naturally preserves the Poincare covariance. Bethe-Salpeter equation of deuteron binding is shown in Fig. 1.11. Solid lines are nucleons and dotted line are intermediate bosons. Square implies the sum of irreducible diagrams. It can be arranged as

$$
M=V+V G_{0} M
$$

where $M$ is the scattering matrix, $V$ is the kernel and $G_{0}$ is the propagator.

Direct calculations of Eq. 1.11 is very complicated and approximations are applied in calculation. Ladder diagrams give the biggest contribution in low $Q$ transfer region. [34] On the other hand, crossed terms cancel some of ladder terms. In the limit of infinite nucleon mass, the sum of ladder terms and crossed terms is the same with ladder terms with a constraint of positive energy for a nucleon. [34] Hence recent calculations use this ladder approximation with positive energy constraints. Fig. 1.12 shows diagrams of ladders and two body currents of meson exchange. Fig. 1.13 shows the diagram of cancellation effect. Two treatments are used for calculation of the cancellation diagram. One is known as RIA(Relativistic Impulse 


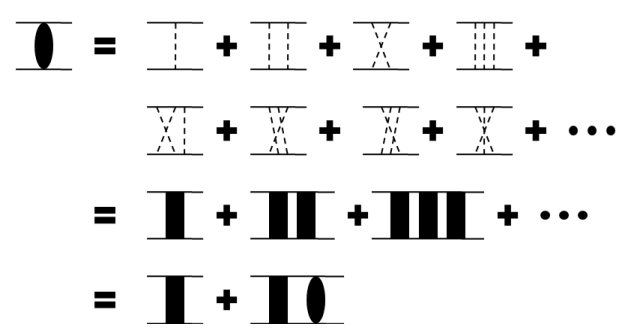

Figure 1.11: Deuteron structure functions. Experimental data are compared with theoretical calculations.

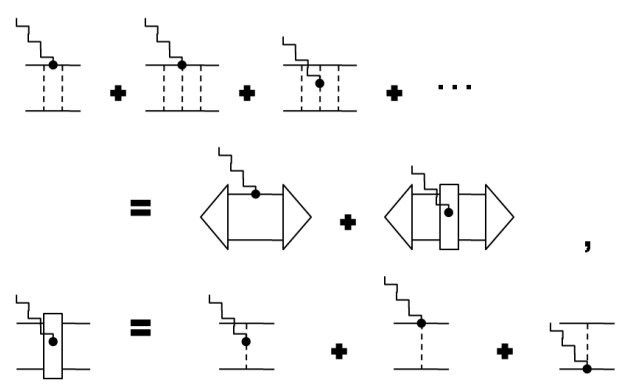

Figure 1.12: Deuteron structure functions. Experimental data are compared with theoretical calculations.

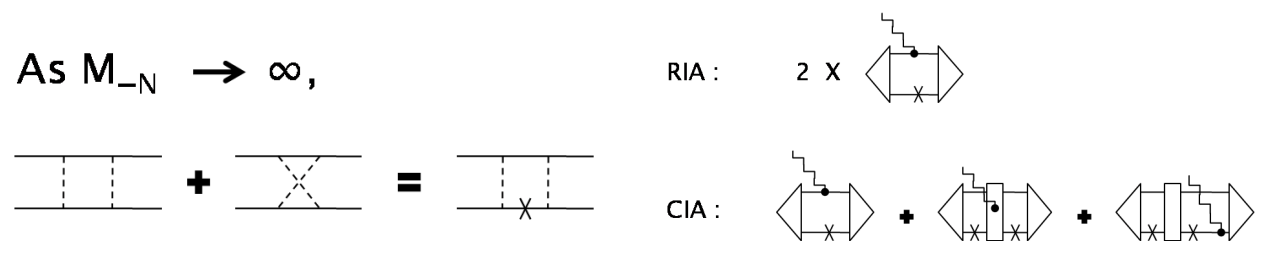

Figure 1.13: Deuteron structure functions. Experimental data are compared with theoretical calculations. 
Approximation) method which do not distinguish two nucleons. Another is known as CIA(Complete Impulse Approximation) which separates two nucleons by the constraint of crossed diagrams.

\section{Relativistic calculations}

Figure 1.14 compares four relativistic calculations together with $A(Q)$ data points. The top panel shows data across the whole region. And the bottom panel is focused on low $Q$ range below $1 \mathrm{GeV} / \mathrm{c}$. The red solid line is made from the propagator formulation using the CIA. Meson exchange currents in Fig. 1.12 are included and additional term of $\rho \pi \gamma$ exchange current is included. [7] The blue long dashed line is also made by propagator formulation and it is generated by the same method of red solid curve except that the RIA is applied and $\rho \pi \gamma$ exchange current is not included. [7] Both of them show consistent agreement with data in the top panel. The big difference in the high $Q$ region is generated from the existence of $\rho \pi \gamma$ current which only contributes at high $Q$ region. The black solid line is one of hamiltonian method calculations and it is quantized in instant form. [29] It includes effects of meson exchange currents in Fig. 1.10. The purple line is one of hamiltonian method calculations and it is quantized in point form. [30] This does not include meson exchange current terms. The existence of meson exchange currents generates big difference in high $Q$ region. The black line shows better agreement than purple line which gives wrong description in high $Q$ region. The calculations of red, blue and black lines are more complete than that of purple line because they included meson exchange currents which give big contribution in high $Q$ region. They tend to agree better with the Mainz data and it is shown in the bottom panel of the plot. On the other hand, purple line agrees better with the Saclay data but it does not describe high $Q$ region well. Thus, theoretical bias would suggest that the correct trend is the Mainz, rather than the Saclay data.

If Mainz data are correct, some relativistic calculations give good agreement across 

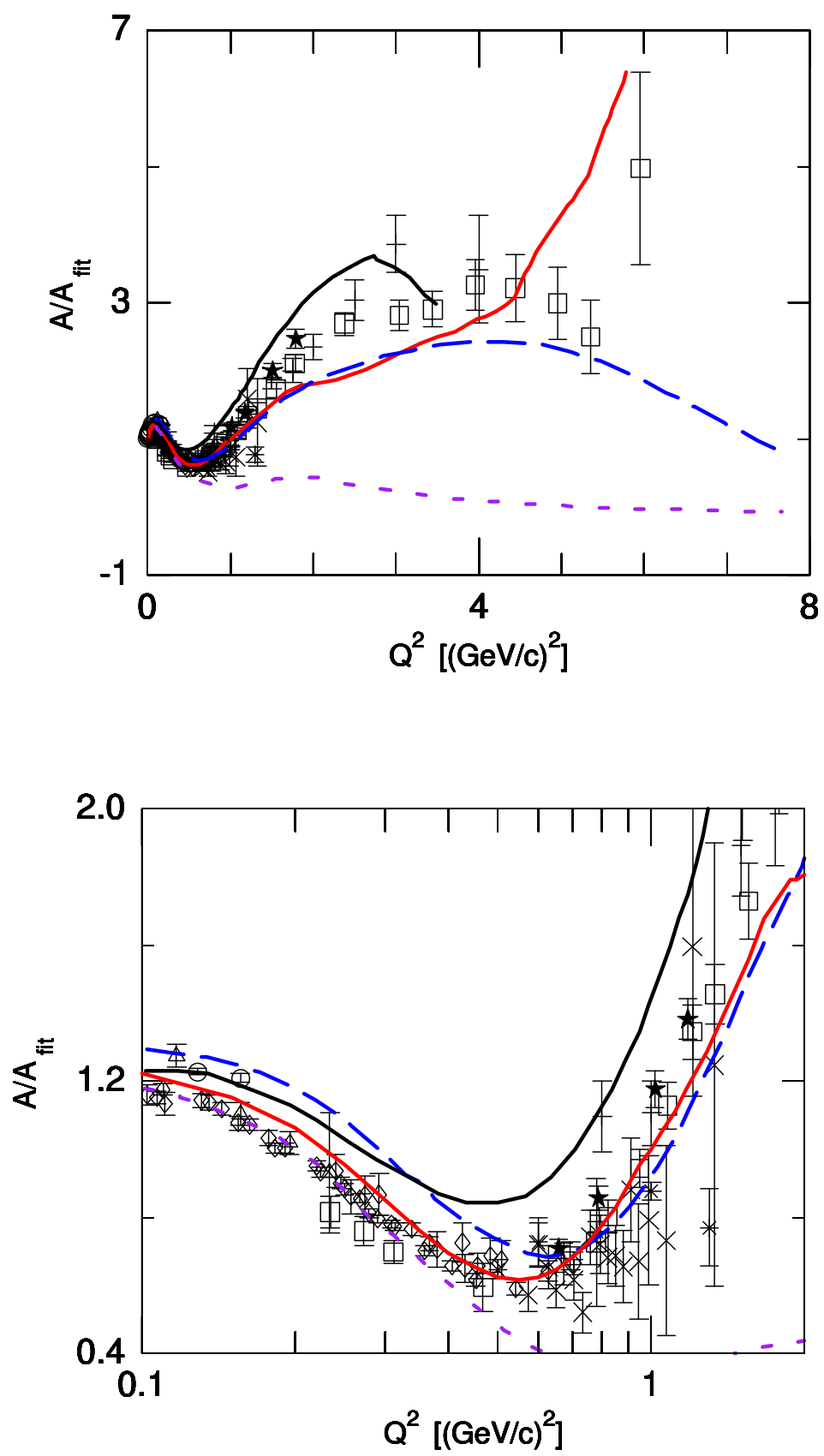

Figure 1.14: The data for $A(Q)$, compared to four relativistic calculations. Top panel shows the behavior across the whole range and bottom panel is focused on low energy region.

CIA (red solid line) and RIA (blue long dashed line) of the propagator method and one body + two body currents (black solid line) and one body currents (purple short dashed line) of the hamiltonian method are drawn together. 
the whole region of $Q$. If Saclay data are correct, no theories give good answer across the whole region. If correct result is between Mainz and Saclay data, CIA gives the best agreement among calculations and ChiPt also gives good description.

\subsection{Experimental Goals and Kinematic Setting}

In this experiment, elastic scattering ${ }^{2} \mathrm{D}\left(e, e^{\prime}\right)^{2} \mathrm{D}$ are measured to obtain $A(Q)$ in the $Q$ range from $0.15 \mathrm{GeV} /$ c to $0.7 \mathrm{GeV} / \mathrm{c}$. The main purpose is to resolve the $10 \%$ discrepancies of $A(Q)$ among the existing data of Mainz, Orsay and Saclay in the $Q$ range from $0.2 \mathrm{GeV} /$ c to $0.4 \mathrm{GeV} / \mathrm{c}$. Precise measurement is aimed with uncertainty of $<1 \%$ statistically and $2-3 \%$ systematically. Beam energy is 685 $\mathrm{MeV}$ and cross sections are obtained across 13 kinematic settings with angles from $14.5^{\circ}$ to $69.0^{\circ}$. These forward angles suppress the contribution of $B(Q)$ term in the region $0.2<Q<0.4 \mathrm{GeV} / \mathrm{c}$ to be less than $1 \%$. In addition, $B(Q)$ fit function is made from world data. Hence accurate data of $A(Q)$ can be extracted after $B(Q)$ fit function is subtracted from obtained cross sections. Carbon and hydrogen elastic scatterings are measured together and compared with world data to ensure that data are well calibrated. Tantalum and aluminum are also measured and they are used for energy calibration and liquid target end cap subtraction. Kinematic settings are summarized in Table 1.2. 
Table 1.2: Kinematic settings.

\begin{tabular}{|c|c|c|c|c|}
\hline & $\begin{array}{c}\mathrm{Q} \\
\mathrm{GeV} / \mathrm{c}\end{array}$ & $\begin{array}{c}\theta \\
\text { (degree) }\end{array}$ & Target & $\begin{array}{c}\text { Momentum } \\
(\mathrm{MeV} / \mathrm{c})\end{array}$ \\
\hline Kin-7 & 0.172 & 14.5 & $\mathrm{H}, \mathrm{D}, \mathrm{C}, \mathrm{Al}, \mathrm{Ta}$ & 663.69 \\
\hline Kin-2 & 0.201 & 17.0 & $\mathrm{H}, \mathrm{D}, \mathrm{C}, \mathrm{Al}, \mathrm{Ta}$ & 663.69 \\
\hline Kin-8 & 0.247 & 21.0 & $\mathrm{H}, \mathrm{D}, \mathrm{C}, \mathrm{Al}, \mathrm{Ta}$ & 663.69 \\
\hline Kin-1 & 0.281 & 24.0 & $\mathrm{H}, \mathrm{D}, \mathrm{C}, \mathrm{Al}, \mathrm{Ta}$ & 663.69 \\
\hline Kin-9 & 0.298 & 25.5 & $\mathrm{H}, \mathrm{D}, \mathrm{C}, \mathrm{Al}, \mathrm{Ta}$ & 663.69 \\
\hline Kin-3 & 0.353 & 30.5 & $\begin{array}{l}\mathrm{C}, \mathrm{Ta} \\
\mathrm{D}, \mathrm{Al} \\
\mathrm{H}, \mathrm{Al}\end{array}$ & $\begin{array}{l}663.69 \\
653.86 \\
623.80\end{array}$ \\
\hline Kin-10 & 0.400 & 35.0 & $\begin{array}{l}\text { C, Ta } \\
\mathrm{D}, \mathrm{Al} \\
\mathrm{H}, \mathrm{Al}\end{array}$ & $\begin{array}{l}663.69 \\
644.32 \\
606.67\end{array}$ \\
\hline Kin-4 & 0.451 & 40.0 & $\begin{array}{l}\text { C, Ta } \\
\text { D, Al } \\
\mathrm{H}, \mathrm{Al}\end{array}$ & $\begin{array}{l}663.69 \\
632.78 \\
586.53\end{array}$ \\
\hline kin-11 & 0.500 & 45.0 & $\begin{array}{l}\text { C, Ta } \\
\mathrm{D}, \mathrm{Al} \\
\mathrm{H}, \mathrm{Al}\end{array}$ & $\begin{array}{l}663.69 \\
620.44 \\
565.69\end{array}$ \\
\hline Kin-5 & 0.551 & 50.5 & $\begin{array}{l}\text { C, Ta } \\
\mathrm{D}, \mathrm{Al} \\
\mathrm{H}, \mathrm{Al}\end{array}$ & $\begin{array}{l}663.69 \\
606.20 \\
542.46\end{array}$ \\
\hline Kin-12 & 0.599 & 56.0 & $\begin{array}{l}\text { C, Ta } \\
\mathrm{D}, \mathrm{Al} \\
\mathrm{H}, \mathrm{Al}\end{array}$ & $\begin{array}{l}663.69 \\
591.50 \\
519.37\end{array}$ \\
\hline Kin-6 & 0.651 & 62.5 & $\begin{array}{l}\text { C, Ta } \\
\mathrm{D}, \mathrm{Al} \\
\mathrm{H}, \mathrm{Al}\end{array}$ & $\begin{array}{l}663.69 \\
573.86 \\
492.79\end{array}$ \\
\hline Kin-13 & 0.700 & 69.0 & $\begin{array}{l}\text { C, Ta } \\
\mathrm{D}, \mathrm{Al} \\
\mathrm{H}, \mathrm{Al}\end{array}$ & $\begin{array}{l}663.69 \\
556.27 \\
467.41\end{array}$ \\
\hline
\end{tabular}




\section{Chapter 2}

\section{Experimental Setup}

\subsection{Overview of Accelerator}

Jefferson Lab(JLab) is an institution to study structures of nuclei, hadrons and quarks with an electron beam accelerator known as CEBAF(Continuous Electron Beam Accelerator Facility) $[35,36]$. Beam electrons can be accelerated up to $6 \mathrm{GeV}$ to probe quarks and nucleus. The beam is polarized and it is possible to probe spin structures either. In this experiment, the main goal is to find the deuteron $A(Q)$ structure function which is irrelevant with the polarization. Hence both of polarization beam are summed in data analysis.

Fig. 2.1 shows the layout of CEBAF. It consists of beam injector, two linacs and several recycle rings. Electrons are generated from a strained GaAs cathode gun. Continuous wave beam are generated and the maximum beam current reaches 200 $\mu \mathrm{A}$. The beam is accelerated in two linacs and each linac has 20 cryomodules with the accelerating gradient of $5 \mathrm{MeV} / \mathrm{m}$ and total $400 \mathrm{MeV}$ per pass. The accelerated beam is delivered into three Halls $\mathrm{A}, \mathrm{B}$ and $\mathrm{C}$ and all Halls can receive the maximum energy beam simultaneously. 


\subsection{Overview of Hall A}

This experiment is operated in Hall A. The lay-out is shown in Fig. 2.2. The beam line is placed up to the center of the hall where targets are located. Electromagnetic interactions are generated at the target and electrons are scattered to every direction. Two High Resolution Spectrometers(HRS) detector systems are placed at angles of kinematic setting and they collect scattered electrons. Both of them

have identical structure and they are designed to detect electrons or hadrons passing through entrance collimators. Each of them consists of QQDQ magnet transportation configuration and main detectors. During this experiment, both spectrometers are set up for electrons. Left arm spectrometer is the main detector and the location is changed by kinematic setting. Right arm is fixed at $24^{\circ}$ during experiment for calibration.

The basic design of HRS is summarized in Table 2.1.

\subsection{The Detector Package}

Detectors are protected by the line-of-sight block and shield hut(SH). The line-ofsight block is a $2 \mathrm{~m}$ concrete block to reduce the muon rate from pion decay. This is located on the top of quadrupole Q1 and Q2. The SH surrounds the detector package and it blocks background radiations from all direction.

Hall A detector packages are drawn in Fig. 2.3. This is the side view of detector stack. Two VDC planes are at the front and it is used to find the trajectory of scattered electron. Scintillators, Cherenkov detectors and pion rejectors are placed behind. Scintillators are triggers for VDC detectors. Other detectors are for particle identifications(PIDs). Each detector can be easily inserted or removed by the necessity of experiment. 


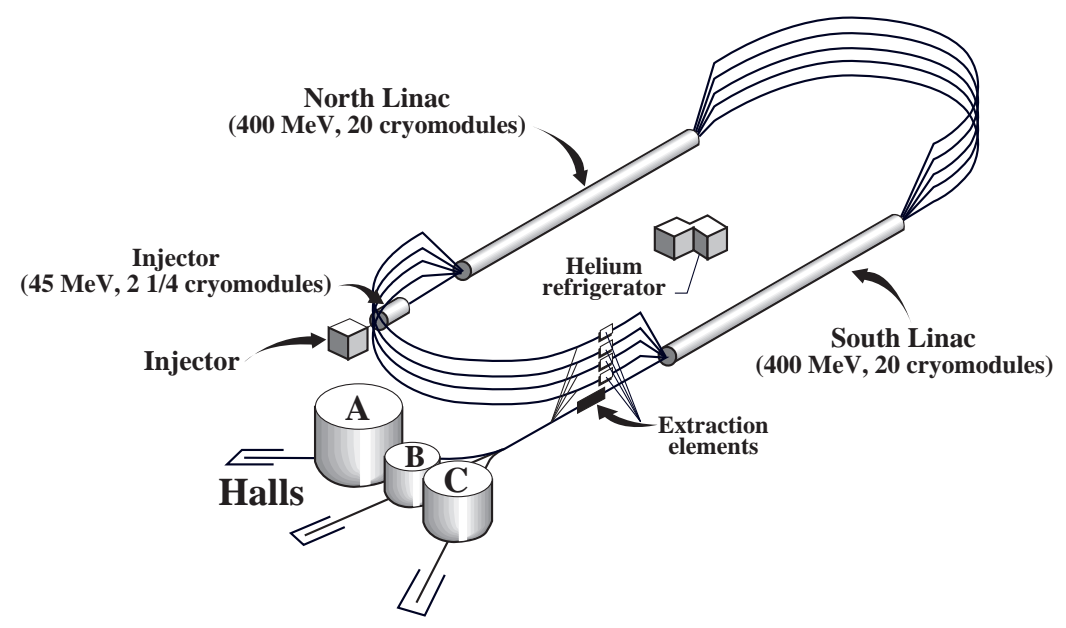

Figure 2.1: Lay-out of CEBAF in JLab. [36]

The electron beam is produced at the injector and then accelerated through the two linear accelerators. Two linear accelerators are connected by several recycle rings.

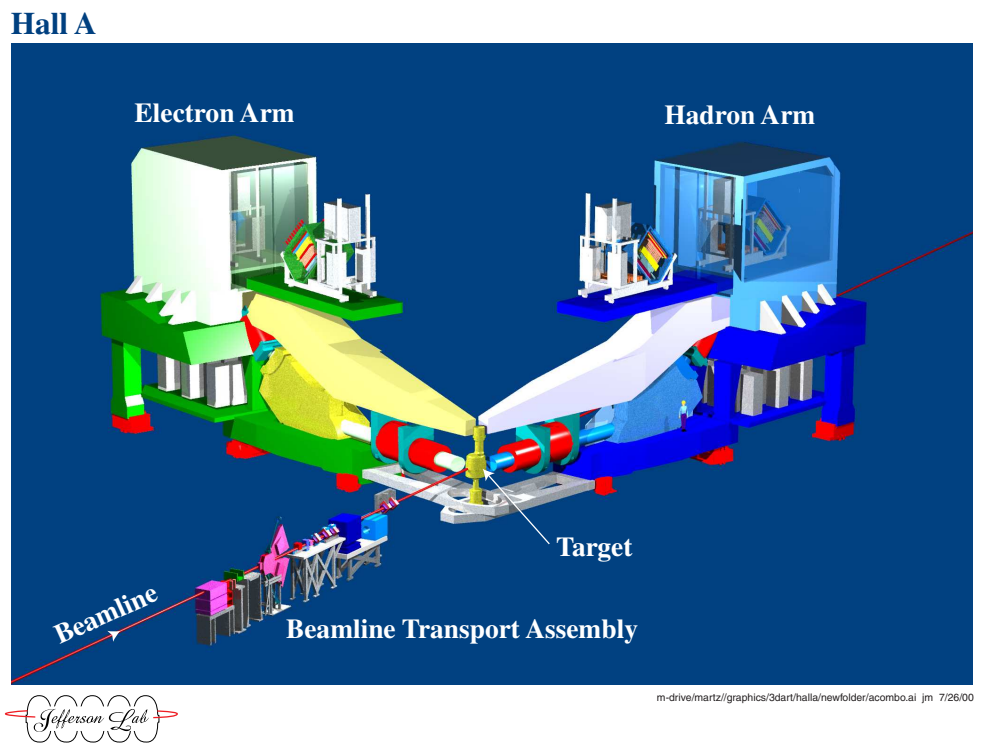

Figure 2.2: Lay-out of Hall A. [37]

The electron beam enters through the beamline and interacts with the target. There are two spectrometers in Hall A and each of them can detect electron or hadron. 

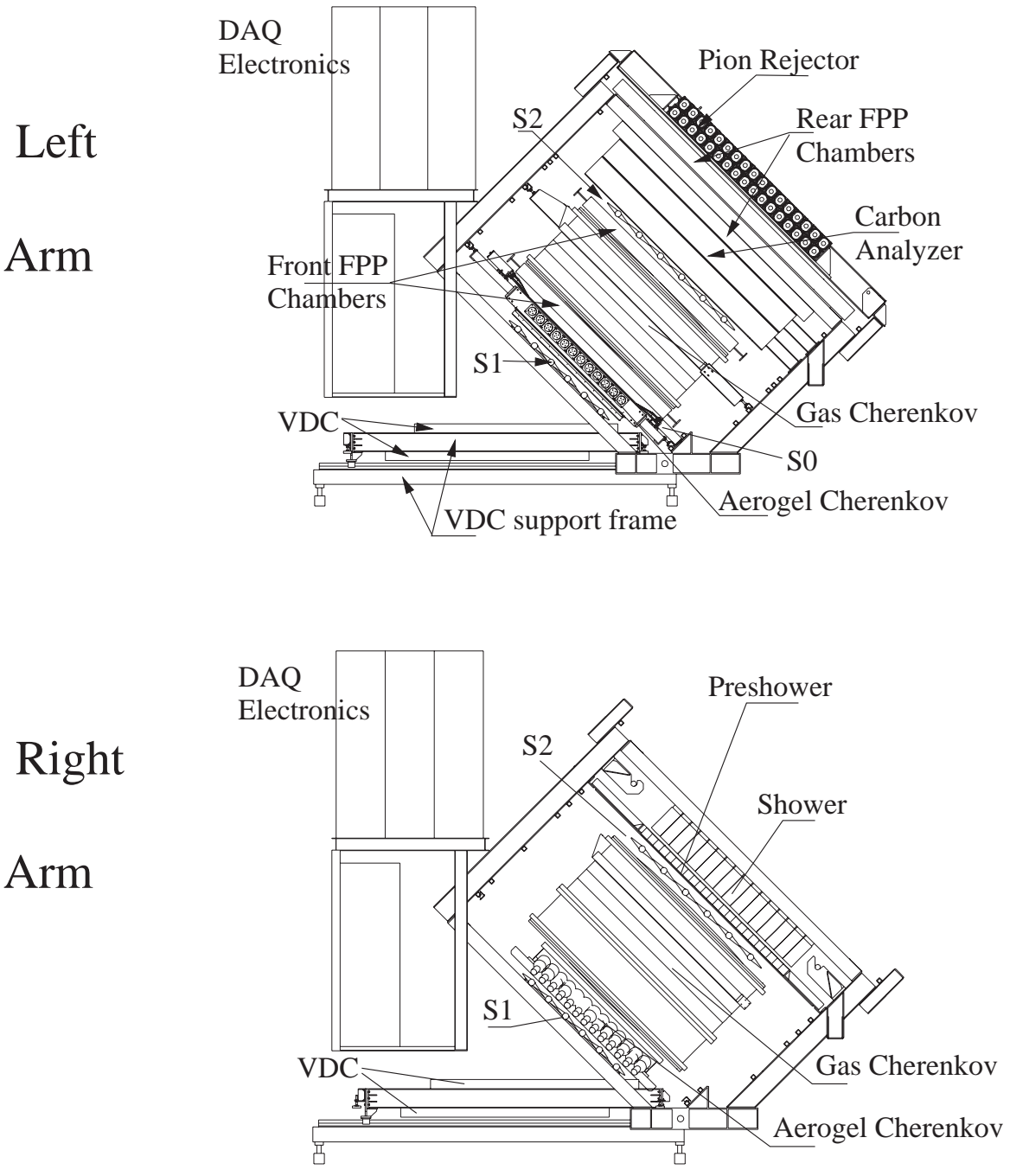

Figure 2.3: Sideview of the detector stack. [36] 
Table 2.1: Main design characteristics of the Hall A High Resolution Spectrometers. [36] The resolution values are for the FWHM.

\begin{tabular}{|l|c|}
\hline Configuration & $\mathrm{QQD}_{n} \mathrm{Q}$ Vertical bend \\
Bending angle & $45^{\circ}$ \\
Optical length & $23.4 \mathrm{~m}$ \\
Momentum range & $0.3-4.0 \mathrm{GeV} / \mathrm{c}$ \\
Momentum acceptance & $-4.5 \%<\delta \mathrm{p} / \mathrm{p}<+4.5 \%$ \\
Momentum resolution & $1 \times 10^{-4}$ \\
Dispersion at the focus (D) & $12.4 \mathrm{~m}$ \\
Radial linear magnification (M) & -2.5 \\
D/M & 5.0 \\
Angular range: HRS-L $\quad$ HRS-R & $12.5^{\circ}-150^{\circ}$ \\
& $12.5^{\circ}-130^{\circ}$ \\
Angular acceptance: Horizontal & $\pm 30 \mathrm{mrad}$ \\
\multicolumn{1}{|c}{ Vertical } & $\pm 60 \mathrm{mrad}$ \\
Angular resolution : Horizontal & $0.5 \mathrm{mrad}$ \\
Vertical & $1.0 \mathrm{mrad}$ \\
Solid angle at $\delta \mathrm{p} / \mathrm{p}=0$, yo $=0$ & $6 \mathrm{msr}$ \\
Transverse length acceptance & $\pm 5 \mathrm{~cm}$ \\
Transverse position resolution & $1 \mathrm{~mm}$ \\
\hline
\end{tabular}

\subsubsection{Triggering}

The trigger logic is constructed from two scintillator planes ( $\mathrm{S} 1$ and $\mathrm{S} 2$ ) and gas Cherenkov detector. Each scintillator plane has six paddles and each paddle is connected with two PMTs. The time resolution per plane is 0.3 ns. A scintillator signal is detected from a paddle with two PMTs' coincidence. 'Good event type' is defined with more than two signals among S1, S2 and gas Cherenkov and the type of trigger efficiency is defined with one signal among three detectors. Every trigger signal is collected into the trigger supervisor module and it starts the DAQ readout. If the signal is accepted by the DAQ, it is re-timed and makes gates for ADCs and TDCs. 


\subsubsection{Tracking}

All information of electron trajectory can be obtained from track records of vertical drift chamber(VDC). The VDC layout is drawn in Fig. 2.4. There are two VDC detectors which are separated by $335 \mathrm{~mm}$ and each has two wire planes called as $\mathrm{U}$ and V. The trajectory of each electron is found by VDC detectors and two positions from two VDCs complete the scattering information. Each wire plane consists of 368 wires spaced with $4.24 \mathrm{~mm}$ and two wire planes $\mathrm{U}$ and $\mathrm{V}$ are placed with $45^{\circ}$ rotation. If an electron passes a VDC wire plane, it disturbs the electromagnetic field around VDC wires and it is attracted to the nearest wire with nearly constant velocity. When it approaches the wire, the particle ionizes the gas in the chamber and the avalanche of electrons and ions are generated. Consequently, one electron makes a cluster of hitting wires. Tracking algorithm [36] picks the first hitting wire and the position of trajectory is determined from the wire location. This has the resolution of wire separation distance. Hence additional information is necessary and the precise position can be obtained from the correction of TDC data which gives drift distance within each wire cell. Fig. 2.5 draws the typical hitting in a VDC wire plane.

\subsubsection{Particle Identification}

Two Particle Identification Detectors (PID) are equipped in HRS during this experiment. The Gas Cherenkov detector is mounted between scintillators S1 and S2. It is filled with $\mathrm{CO}_{2}$ at atmospheric pressure. The detector is connected with 10 PMTs. Because the Cherenkov radiation threshold is proportional to the mass of passing particle, it separates $\pi^{-}$particles(criteria : $4.8 \mathrm{GeV} / \mathrm{c}$ ) from electrons $(17$ $\mathrm{MeV} / \mathrm{c}$ ). During this experiment, the maximum momentum of scattered electron is $685 \mathrm{MeV}$ and $\pi^{-}$events are easily removed.

Behind scintillator S2, two shower detectors are positioned. [38] Each consists of lead glass blocks. Lay-outs of two shower detectors are drawn in Fig. 2.6. In two 


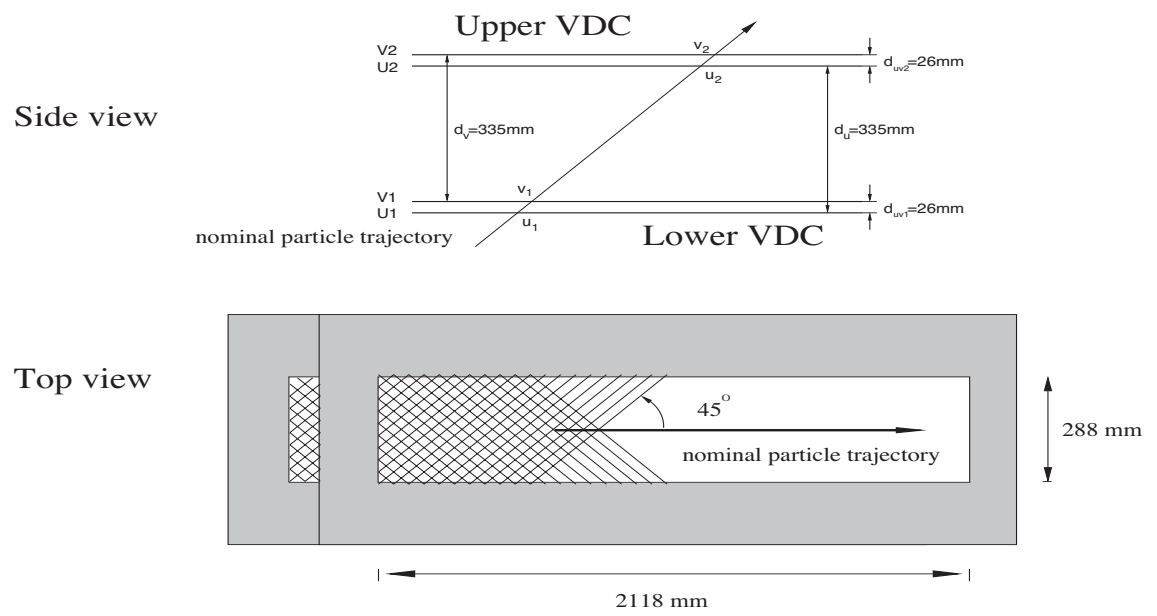

Figure 2.4: Lay-out of a pair of Vertical Drift Chambers. [36]

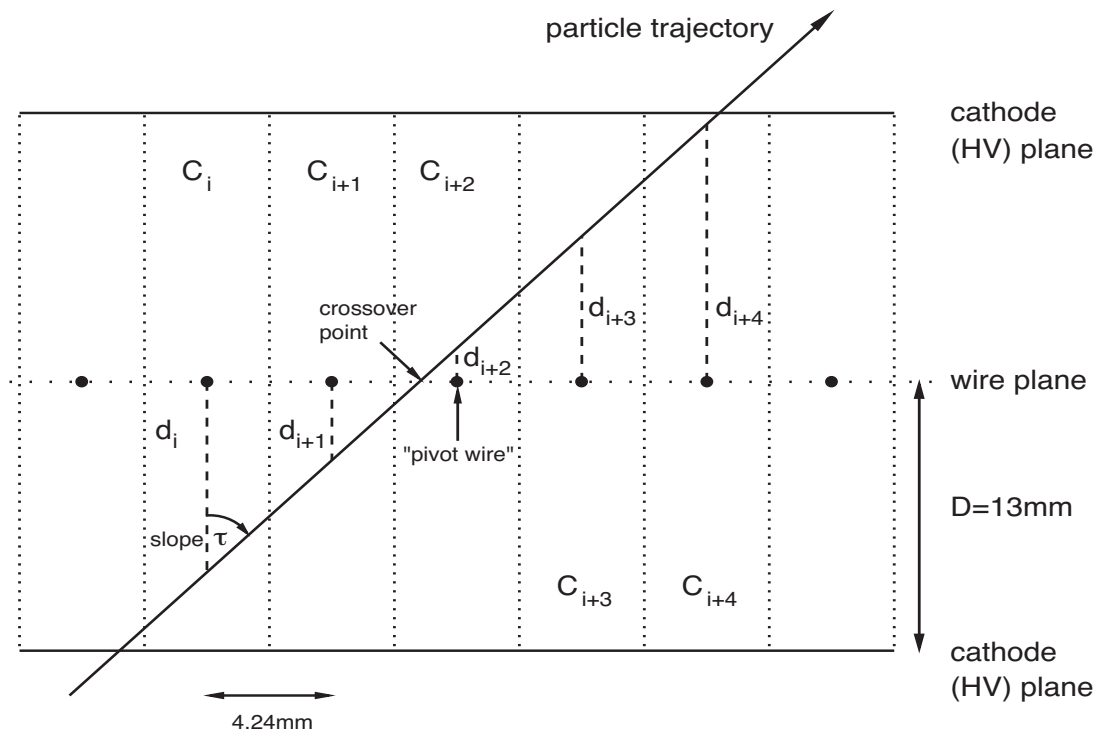

Figure 2.5: A typical cluster of hits in a VDC plane. [36] The HV planes are held at a potential of approximately $-4 \mathrm{kV}$, while the wires are effectively grounded. 


\section{HRS-L}
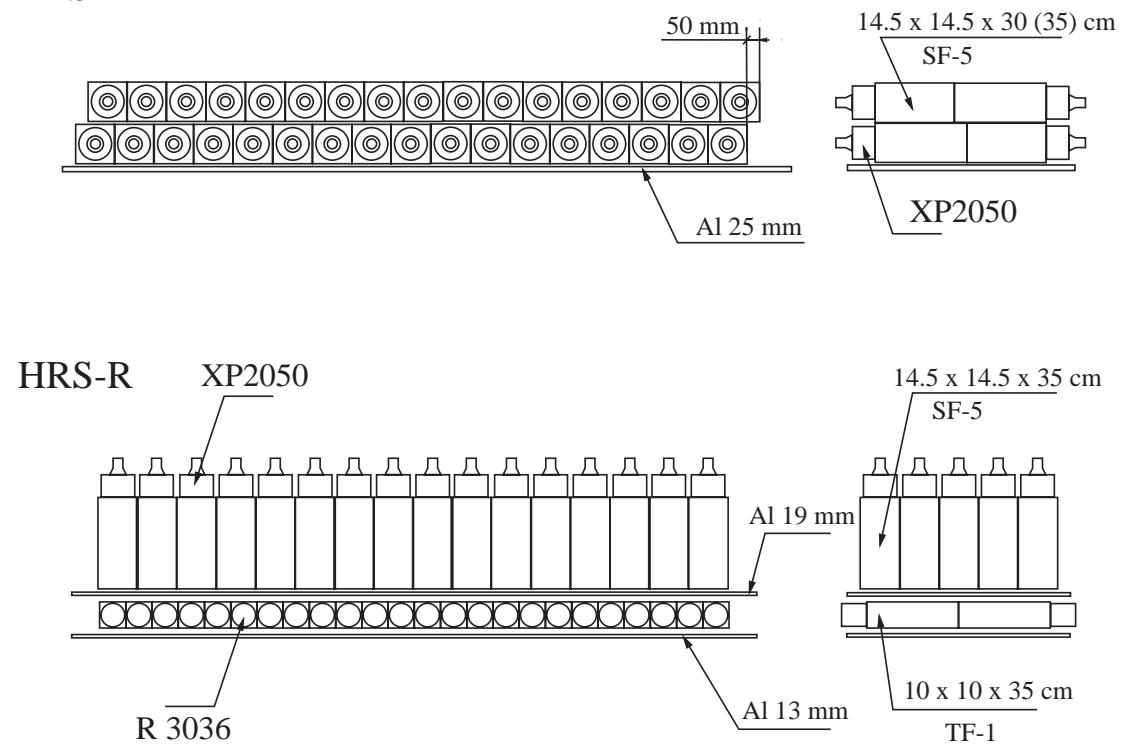

Figure 2.6: Schematic lay-out of part of the shower detectors in HRS-L (top) and HRS-R (bottom). [36] Particles enter from the bottom of the figure.

HRSs, shower detectors are mounted with different directions. The shower detector in left arm has shorter distance, so the resolution is not as good as the one in right arm. $R_{s h}$, the particle identification parameter, is defined as

$$
R_{s h}=\frac{E_{\text {tot }}}{p} \times \frac{\ln \left(E_{\text {presh }}\right)}{\ln \left(E_{\text {ave }}\right)},
$$

where $E_{t o t}$ is the total energy deposited in the shower detector, $p$ is the particle's momentum, $E_{\text {presh }}$ is the energy deposited in the front layer and $E_{\text {ave }}$ is the average energy deposited by an electron with momentum $p$.

Pion suppression efficiency above $2 \mathrm{GeV} / \mathrm{c}$ is known as $98 \%$. 


\subsubsection{Collimator}

Each spectrometer has an entrance collimator which is approximately $1 \mathrm{~m}$ away from the target. In the kinematics of this experiment, cross sections and event rates are quite high. Hence a small collimator of 2(1) msr is added in front of the pre-existing left(right) arm collimator. It has several pin holes around the central hole and they can be used for the calibration check. The draft of left arm collimator is drawn in Fig. 2.7. There is a large square hole in the center and there are several pin holes around the center.

\subsection{Beam Energy Measurement}

There are two methods to measure the absolute beam energy. [39] One is Arc method which is used in this experiment and another is eP measurement. The Arc method, which is known as Tiefenbach method, measures the deflection of the beam in the arc section of the beam line. There are eight dipoles in the line and the nominal bend angle of the beam in the section is $34.3^{\circ}$. The momentum of the beam can be calculated from the magnetic field in the dipoles and the resulting bend angle. It is given as

$$
p=k \frac{\int \vec{B} \cdot \overrightarrow{d l}}{\theta}
$$

The eP method [39, 40] measures the energy from kinematic property of elastic

$p\left(e, e^{\prime}\right) p$ interaction. In elastic scattering kinematics, the beam energy can be written by Eq. 2.2.

$$
E=M_{p} \frac{\cos \left(\theta_{e}\right)+\sin \left(\theta_{e}\right) / \tan \left(\theta_{p}\right)-1}{1-\cos \left(\theta_{p}\right)}+O\left(m_{e}^{2} / E^{2}\right)
$$

The polyethylene(CH2) is the target and the Cherenkov detector, the silicon-strip detectors(SSD) and scintillator detectors are used.

Both methods show good agreement with uncertainties less than $0.03 \%$. 


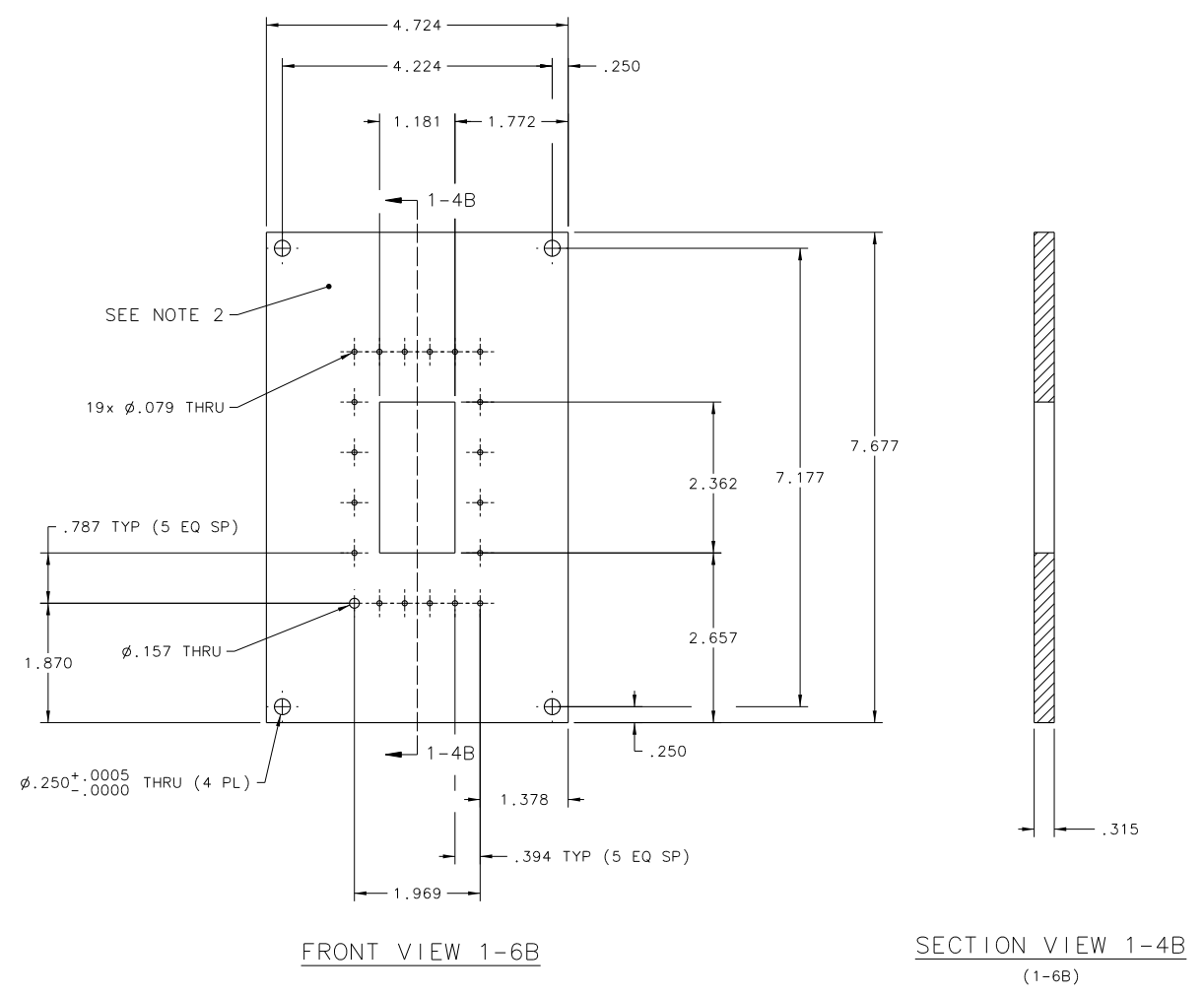

Figure 2.7: Draft of the left arm collimator. This has small 2 msr area. 


\subsection{Beam Position Measurement}

There are two Beam Position Monitors (BPMs) at $6.545 \mathrm{~m}$ and $0.0187 \mathrm{~m}$ upstream of the target. They can measure the relative beam position within $100 \mu \mathrm{m}$. The absolute position can be obtained after the beam calibration job known as bulls eye scan. It gives the absolute positions of wire scanners and the absolute beam position are determined consequently. The position at the target is extrapolated from the values of two monitors.

For liquid targets, the raster has been turned on. During this experiment, the raster size has been maintained with $3 \times 3 \mathrm{~mm}$. BPM is not enough fast to trace the position of each event. To find the position of each event, the raster raw current can be used. The raster raw current determines the direction and the position of raster and it can be related with the beam position by linear equation. The raster calibration job [41] compares the raster raw current with the beam average position obtained from BPMs and it extracts the rastered beam position of each event.

\subsection{Beam Current Measurement}

The beam Current Monitor (BCM) consists of two RF cavities and a Unser monitor. It measures the relative beam current during the experiment without interference. A calibration is necessary to obtain the absolute value. In calibration job, the beam is sent to the Unser monitor with known current and two reasonant RF cavities on either side of Unser monitor give the voltage values. The equation relating the beam current with the voltage can be extracted. The linearity is preserved with $1 \%$ uncertainty for currents from $10 \mu \mathrm{A}$ to above $200 \mu \mathrm{A}$. Each cavity signal is divided into three channels. One signal is preserved and two other signals are amplified with $\times 3$ and $\times 10$.

In this experiment low currents down to $1 \mu \mathrm{A}$ are required because of high cross section. Hence a beam silver calorimeter is used for the calibration instead of Unser 
monitor at low current. It is known that the silver calorimeter can calibrate the beam current from a few hundred nA to a few $\mu \mathrm{A}$ with $0.5 \%$ uncertainty. [42] In a calibration run, beam goes into the silver calorimeter and deliver the beam power to the slug. It changes the temperature of the slug whose heat capacity is precisely determined. From the temperature change, the beam current can be extracted.

Fig. 2.8 shows the draft of silver calorimeter.

\subsection{Target System}

Hall A targets are contained in a scattering chamber of stainless-steel. The diameter is $1037 \mathrm{~mm}$ and there exist a cutout region which is covered with thin $0.38 \mathrm{~mm}$ aluminum foils on each side of the beam from $12.5^{\circ}$ to $167.5^{\circ}$. All targets are mounted on the ladder type chamber. Especially cryogenic targets are attached with the cryogenic system such as cooling, gas handling, temperature and pressure monitoring. There are three independent cryogenic target loops of liquid hydrogen (LH2), liquid deuterium (LD2) and gaseous helium. Each target has two types of length $4 \mathrm{~cm}$ and $15 \mathrm{~cm}$ cells and the cells are made of aluminum. The end wall of cell has $2 \mathrm{~cm}$ curvature radius. Details of each target can be found in [36].

Fig. 2.9 are pictures of liquid target system and solid targets. Liquid targets in top panel are contained in aluminum cells and back regions are round shape. Solid targets in bottom panel are attached to the aluminum frame and they are placed in center region. Environment of liquid target systems is always monitored with pressure transducers at several locations. Target densities are maintained with the uncertainty less than $0.1 \%$. LH2 is operated with $19 \mathrm{~K}$ and $0.17 \mathrm{MPa}$ and LD2 is operated with $22 \mathrm{~K}$ and $0.15 \mathrm{MPa}$. Their densities are $0.0723 \mathrm{~g} / \mathrm{cm}^{3}$ and $0.167 \mathrm{~g} / \mathrm{cm}^{3}$. The operating condition for ${ }^{4} \mathrm{He}$ is $6.3 \mathrm{~K}$ at $1.4 \mathrm{MPa}$ and $6.3 \mathrm{~K}$ at $1.1 \mathrm{MPa}$ for ${ }^{3} \mathrm{He}$. Densities of helium targets are $0.13 \mathrm{~g} / \mathrm{cm}^{3}$ and $0.07 \mathrm{~g} / \mathrm{cm}^{3}$.

There are three dummy targets to measure contributions of cell windows, made of 


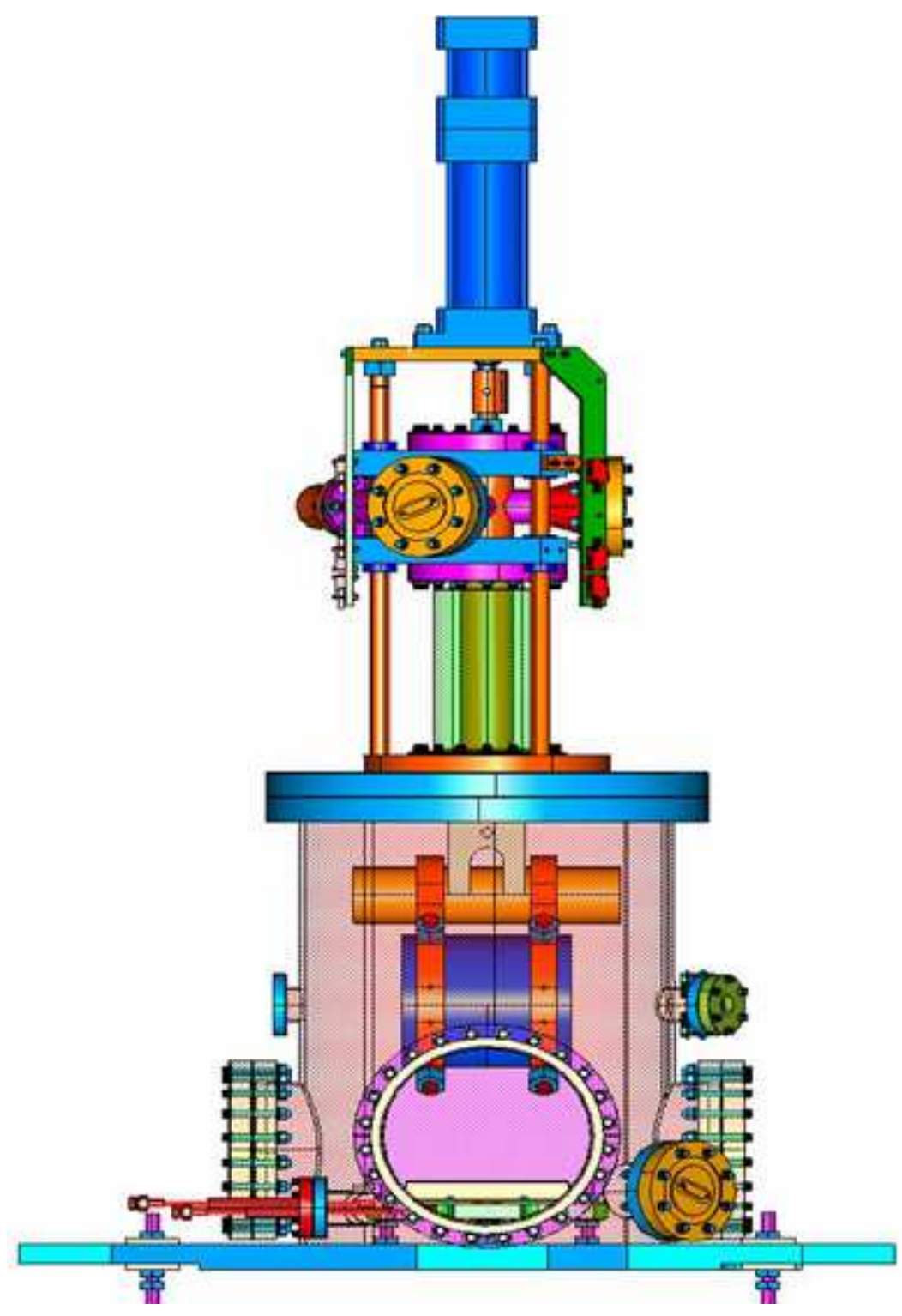

Figure 2.8: Draft of silver calorimeter. Beam goes to the slug and changes the temperature of the slug. The beam current can be calculated from the temperature change. 


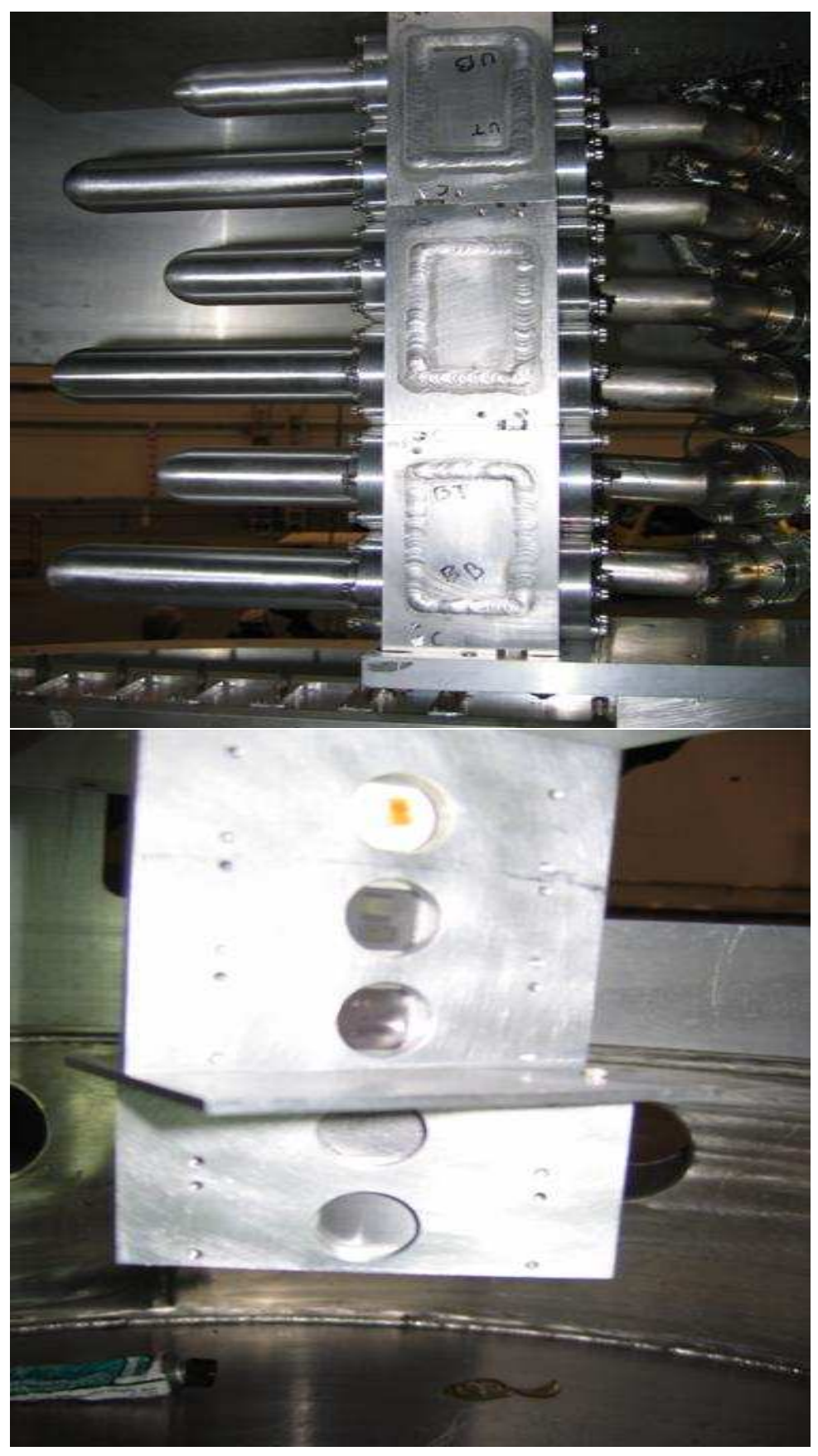

Figure 2.9: Hall A targets. [37] The top panel shows liquid targets and the bottom panel shows solid targets. 
Aluminum. It is two lined up aluminum planes separated by a distance of $4 \mathrm{~cm}, 10$ $\mathrm{cm}$ and $15 \mathrm{~cm}$ to be matched with liquid target cells.

For this experiment, five target materials, $4 \mathrm{~cm} \mathrm{LH2,} 4 \mathrm{~cm} \mathrm{LD} 2,4 \mathrm{~cm}$ dummy, ${ }^{12} \mathrm{C}$ and ${ }^{181} \mathrm{Ta}$, are used.

\subsection{Data Acquisition}

If a scattered electron satisfies the trigger condition, the trigger supervisor opens the gate and starts recording. This makes an event which contains all information of scattering interaction and it is inserted into the CEBAF On-line Data Acquisition System(CODA) [43]. For each event, corresponding data from each read-outcontroller(ROC) are collected together. There are four type components in raw data.

1. Data of scattered electron information (trajectory, momentum, etc)

2. Scalers (normalization factor like beam charge, trigger, etc)

3. Information of kinematic setting : Experimental Physics and Industrial Control System (EPICS)

4. Specific information of the detector setting (detector map)

Analyzer [44] is a Hall A tool for the decoding of raw data and it generates ntuples which can be analyzed with software ROOT. 



\section{Chapter 3}

\section{Data Analysis Part I : Detector Efficiencies and Target Variable Calibrations}

\subsection{Overview}

Each process of data analysis is explained in this chapter and next one. The main objective of this experiment is obtaining data of deuteron structure function $A(Q)$ which can be extracted from the values of electromagnetic cross sections of ${ }^{2} \mathrm{D}\left(e, e^{\prime}\right)^{2} \mathrm{D}$ and $B(Q)$ by Eq. 1.8. Since values of $B(Q)$ are fitted from world data, the process of data analysis is focused on calculating electron-deuteron cross sections.

If an electron interacts with a nucleus, the energy of scattered electron is determined from the beam energy and the scattering angle and it is given as

$$
\frac{E^{\prime}}{E}=\frac{1}{1+\frac{2 E}{M} \sin ^{2} \frac{\theta}{2}} .
$$


Consequently $\frac{d \sigma}{d \Omega}\left(\theta, Q^{2}\right)$ is the meaningful differential cross section in elastic scattering and experimentally it is given as

$$
\begin{aligned}
\frac{d \sigma}{\mathrm{d} \Omega}\left(\theta, Q^{2}\right) & =\frac{N_{f} \times \varepsilon_{e f f}}{N_{i} \times N_{t g}} \times \frac{1}{\mathrm{~d} \Omega} \\
& =N_{f} \times \varepsilon_{\text {eff }} \times \frac{e}{\int I(t) \mathrm{d} t} \times \frac{A_{\mathrm{tg}}}{\int \rho \mathrm{d} l \times N_{A}} \times \frac{1}{\mathrm{~d} \Omega},
\end{aligned}
$$

where $N_{i}$ is the number of beam electrons hitting the target, $N_{\text {tg }}$ is the number of target nucleus contained in the target per unit area, $N_{f}$ is the number of detected electrons after scattering, $\varepsilon_{e f f}$ is the total efficiency of detectors, $e$ is the electron charge, $I(t)$ is the beam current, $N_{A}$ is the Avogadro's Number, $A_{\text {tg }}$ is the atomic mass of the target and $\rho$ is the density of target.

Data analysis to obtain deuteron cross sections is processed with the following steps and the lay-out is drawn in Fig. 3.1.

1. Events are reconstructed from raw data and essential cuts are applied to select good events only.

2. Efficiency of each detector is obtained and the total detector efficiency $\varepsilon_{\text {eff }}$ is given by Eq. 3.3.

$$
\varepsilon_{\text {eff }}=\mathrm{PS} \times\left(1-\mathrm{dt}_{e}\right) \times\left(1-\mathrm{dt}_{c}\right) \times \varepsilon_{\text {tracking }} \times \varepsilon_{\text {trigger }},
$$

where PS is the prescale factor, $\mathrm{dt}_{e}$ is the electronics dead time, $\mathrm{dt}_{c}$ is the computer dead time, $\varepsilon_{\text {tracking }}$ is the tracking efficiency and $\varepsilon_{\text {trigger }}$ is the trigger efficiency.

3. Target variables describing the trajectory of a scattered electron are calibrated.

4. Normalizration factors, $N_{i}$ and $N_{\mathrm{tg}}$, are found after several calibrations of beam current, target length correction and target density correction.

5. The yield of elastic scattering is extracted and the acceptance is calculated. 
6. The cross section at each kinematics is obtained from Eq. 3.2.

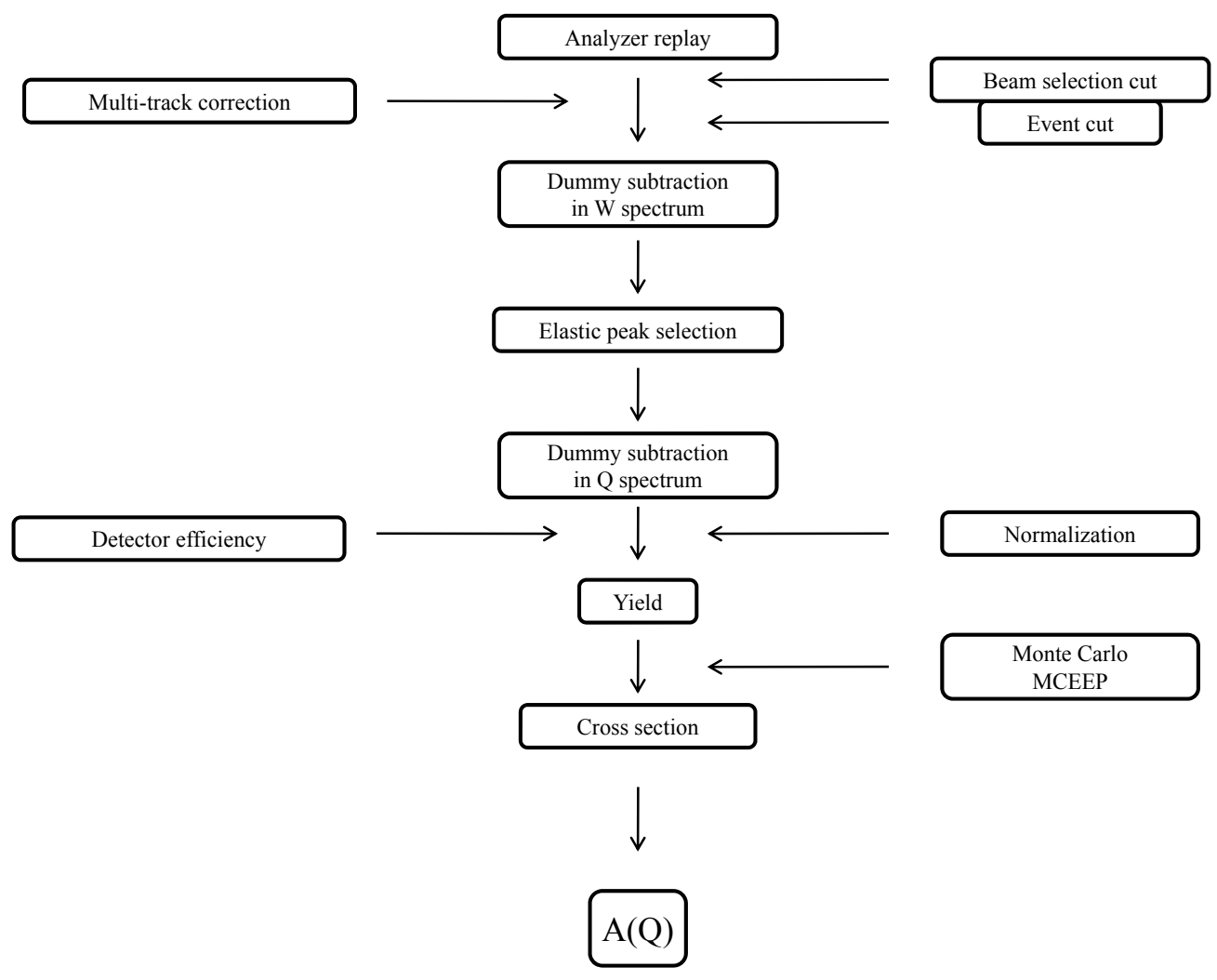

Figure 3.1: Lay-out of data analysis

\subsection{Event Selection}

This experiment has a goal to resolve the discrepancy of $\sim 10 \%$ between other experiments so precision measurements are required. Hence we tried to select only stable beam regions by applying two cuts of BCM cut and BPM cut. BCM cut and BPM cut allow accurate information of the target density and target variables. These cuts differ with event selection cuts such as Cherenkov sum cut and good event cut, since they are applied to beam properties and normalization factors either. 


\subsubsection{Event reconstruction}

All information of scattering interaction is available after Analyzer, which is a Hall A standard decoding software [44], transforms detector data into computer data known as the 'event'. The program is based on ROOT which is developed by CERN for data analysis of nuclear physics and particle physics. Analyzer generates a 'root' formatted file that can be read in ROOT. The root file contains data of events in the type of ROOT trees, ntuples and histograms. All information of each event are stored in a column of ROOT tree. Replay procedure of Analyzer is as following [36].

- Analyzer reads raw data of DAQ system and then decodes data from several detectors which are designated by the input file.

- It reconstructs tracks of electrons by implemented algorithm and finds trajectories at the focal plane.

- By given optics matrix, focal plane variables are transformed into target variables which have scattering information.

\subsubsection{Beam selection cut 1 : BCM cut}

Target density is one of normalization factors which is directly related with the cross section. When the electron beam is passing through a liquid target, some amount of beam power is delivered to the target materials and boil molecules nearby the beam passage. Consequently, the target density is decreased. The decrease is proportional to the beam current and the target density becomes a function of the beam current as

$$
\rho=\rho(I)=\rho(I, \rho(0))
$$

where $I$ is the beam current and $\rho(0)$ is the original target density with no beam. During an experimental run, the beam can be stopped with several reasons. Whenever the beam is turned back on, the target density changes from $\rho(0)$ to $\rho(I)$. and 
the target density is uncertain for a short time.

In this analysis, BCM cut is introduced to remove this uncertainty. BCM cut excludes the data during short period right after the beam is turned back on. BCM data during a run are stored as scaler type and scaler variables are stored at each period. On the other hand, kinematic variables are stored at each event. One period of JLab Hall A scaler system is 4 seconds. When a beam region is selected by a BCM cut, the first event and the last event should be synchronized to a scaler period because the beam current and accumulated charge are known only at the end of each period. Trigger rates and event rates during this experiment are very high in general and the maximum trigger rate reaches $\sim 200 \mathrm{kHz}$. For a run with $10 \mathrm{kHz}$ event rate, there are 40000 events in a scaler period and maximum 40000 events could be lost in synchronization. If a BCM cut consists of several regions, each region requires synchronization and the event loss increases. Not only BCM cut but BPM cut is also applied for the beam selection. Hence the data statistics should be sacrificed if Hall A scaler is used for this experiment. Instead, ROC10/11 are used for the beam normalization jobs and selection cuts.

ROC10/11 can play a role of fast scaler. They are recorded with $30 \mathrm{~Hz}$ and redundant to have all the information of scalers. Moreover ROC10/11 have fast $100 \mathrm{kHz}$ clock $^{1}$. So using ROC10/11 diminishes the error of beam charge and preserves the good statistics. ROC10/11 were designed to have information of polarization and have been used for experiments using beam polarization. ROC10 is made for right arm and ROC11 is for left arm.

ROC10 and scaler are compared in Fig. 3.2. The $x$ axis is time scale and the $y$ axis is BCM value which is proportional to the accumulated beam charge. The top panel is made from ROC10 and the bottom panel is made from scaler. While ROC10 has many points, scaler have only several points because of slow rate. A period of scaler and ROC10 corresponds to each dot and the beam current and accumulated charge

\footnotetext{
${ }^{1}$ Scaler also has $100 \mathrm{kHz}$ clock
} 
are known at each point.

Fig. 3.3 shows a BCM cut from a run. The $x$ axis is time and the $y$ axis is the beam current. In top panel, small values near 0 indicate that the beam was off during the period. The bottom panel is generated after the BCM cut is applied to the top plot. The zero current region and short period right after beam-back are removed in bottom panel.

Fig. 3.4 shows ROC11 event over time. The $x$ axis is event identification number in time order and the $y$ axis is time of (-) helicity. Because ROC11 is recorded at each period, time values for a period are all the same and points look like a line in the plot. The length of first horizontal line is the same with other lines after some periods and it confirms that the first event right after the beam is turned on is synchronized at the initial time of a ROC11 period. The length of lines are different because the $y$ axis is time of (-) helicity and they remain the same during $(+)$ helicity period [45].

\subsubsection{Beam selection cut 2 : BPM cut}

The deuteron $A\left(Q^{2}\right)$ function varies sensitively over $\mathrm{Q}$ in the region of $Q^{2}<1.0$ $(\mathrm{GeV} / \mathrm{c})^{2}$. Old experimental data of $A\left(Q^{2}\right)$ suggest that values can vary $\sim 10^{5}$ times from the minimum in the $Q^{2}$ range of this experiment. It is seen in Fig. 1.3. Hence accurate information of $Q^{2}$ is very important in the cross section calculation. The horizontal beam position, $x_{\text {beam }}$, is one of variables that can generate changes to the kinematic $Q^{2}$ setting. Different beam positions alter the scattering angle and results in the change of $Q^{2}$. The raster is turned on during this experiment and it varies $x_{\text {beam. }}$. So the raster calibration job, which is explained in section 3.5.4, is required for the accurate $x_{\text {beam }}$ and $Q^{2}$. However unstable beam regions

could generate poor results of raster calibration. Hence the BPM cut over $x_{\text {beam }}$ is introduced to extract stable beam regions. This cut is applied to the beam position spread right after the beam is turned on back. Fig. 3.5 shows two contour plots of 

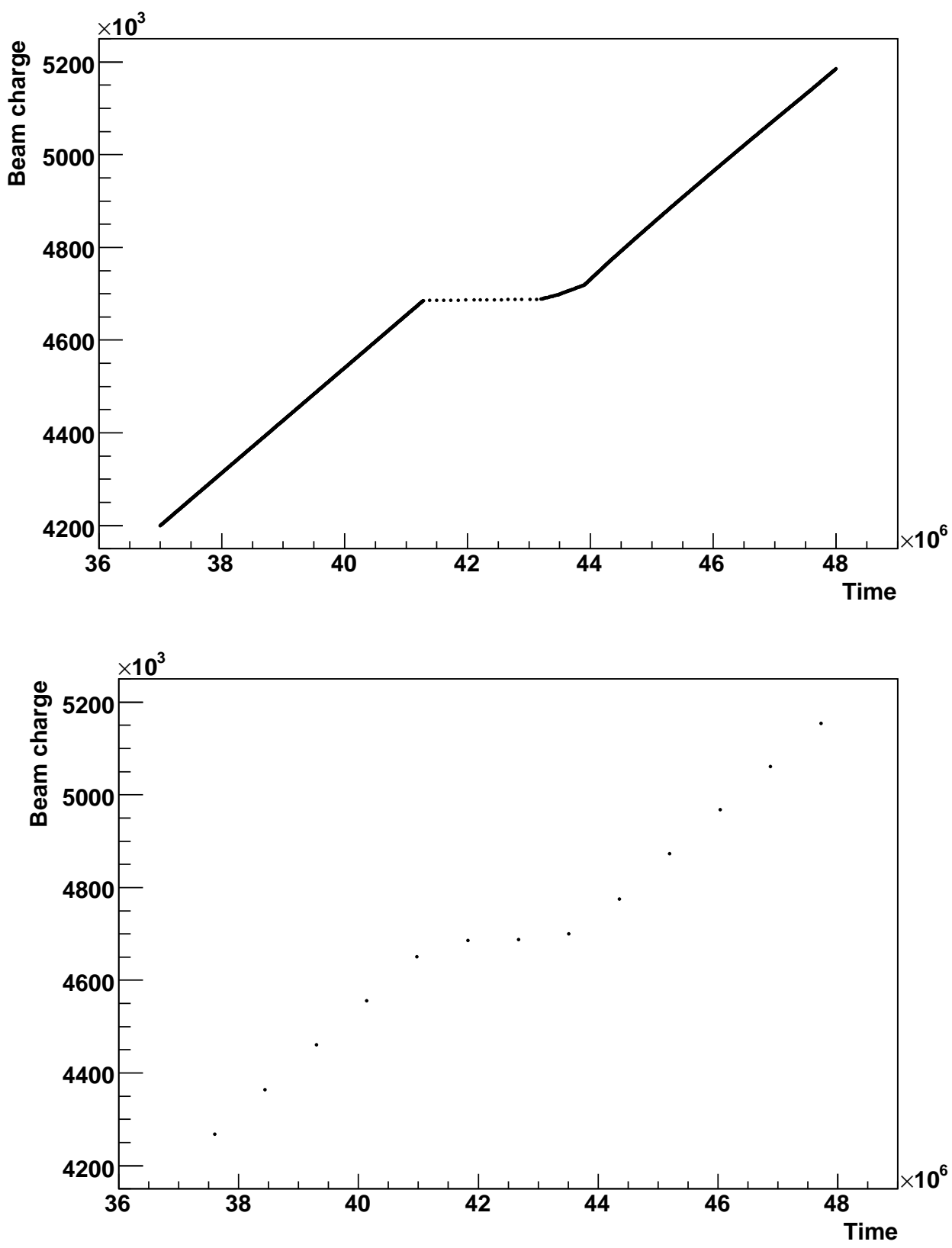

Figure 3.2: Charge accumulation vs. time. Data points from ROC10 are drawn in top panel and ones from scaler are in bottom panel. Because ROC10 is much faster than scaler, there are much more points in top panel. 

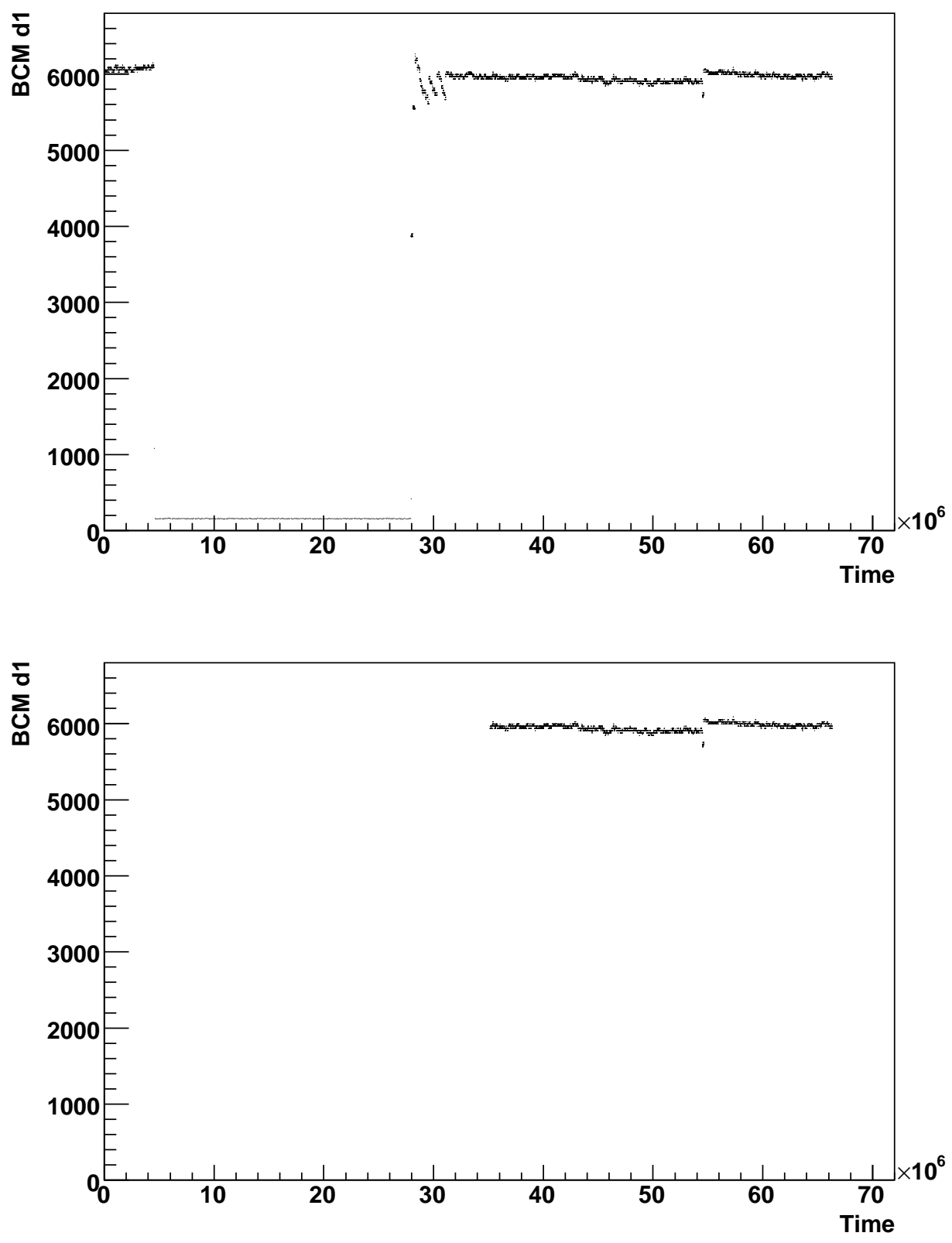

Figure 3.3: Beam current vs. time from ROC11 data. Zero current points in top panel are removed in bottom panel after the BCM cut is applied. 


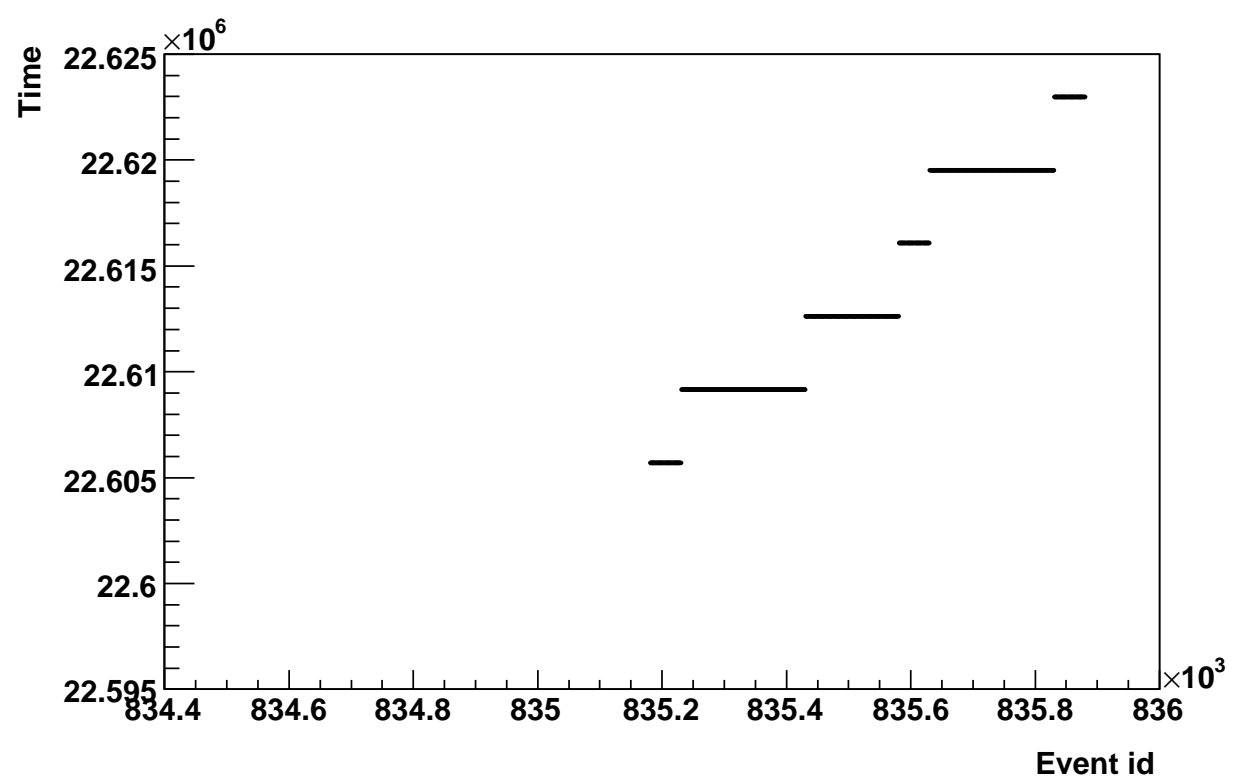

Figure 3.4: Time vs. event id from ROC11 data. After the beam is turned on back, the first event is placed at the initial time of a ROC11 period. Time values are discrete because ROC11 data are recorded at each period.

$x_{\text {beam }}$. The $x$ axis is the event index by time and the $y$ axis is $x_{\text {beam }}$ in meter. The raster is turned on with the size of $3 \mathrm{~mm} \times 3 \mathrm{~mm}$. The top plot is an example of stable runs. It is shown as closed curve with the depth of $3 \mathrm{~mm}$ in contour plot and beam positions show uniform distribution. On the contrary, the bottom plot is an example of runs that have beam trips. Several abrupt changes of $x_{\text {beam }}$ are shown. So the depth is larger than $4 \mathrm{~mm}$.

Distributions of two kinematic variables, $W$ and $Q^{2}$, are compared in Fig. 3.6. They are made from two different regions in bottom plot of Fig. 3.5 and are drawn with two colors. The blue line represents events between two blue vertical lines and the red line represents events between two red vertical lines. The only kinematic difference between them is $x_{\text {beam }}$. The top plot is the distribution of $W-M_{\mathrm{tg}}$ and the bottom plot is the distribution of $Q^{2}$. The total number of each distribution 

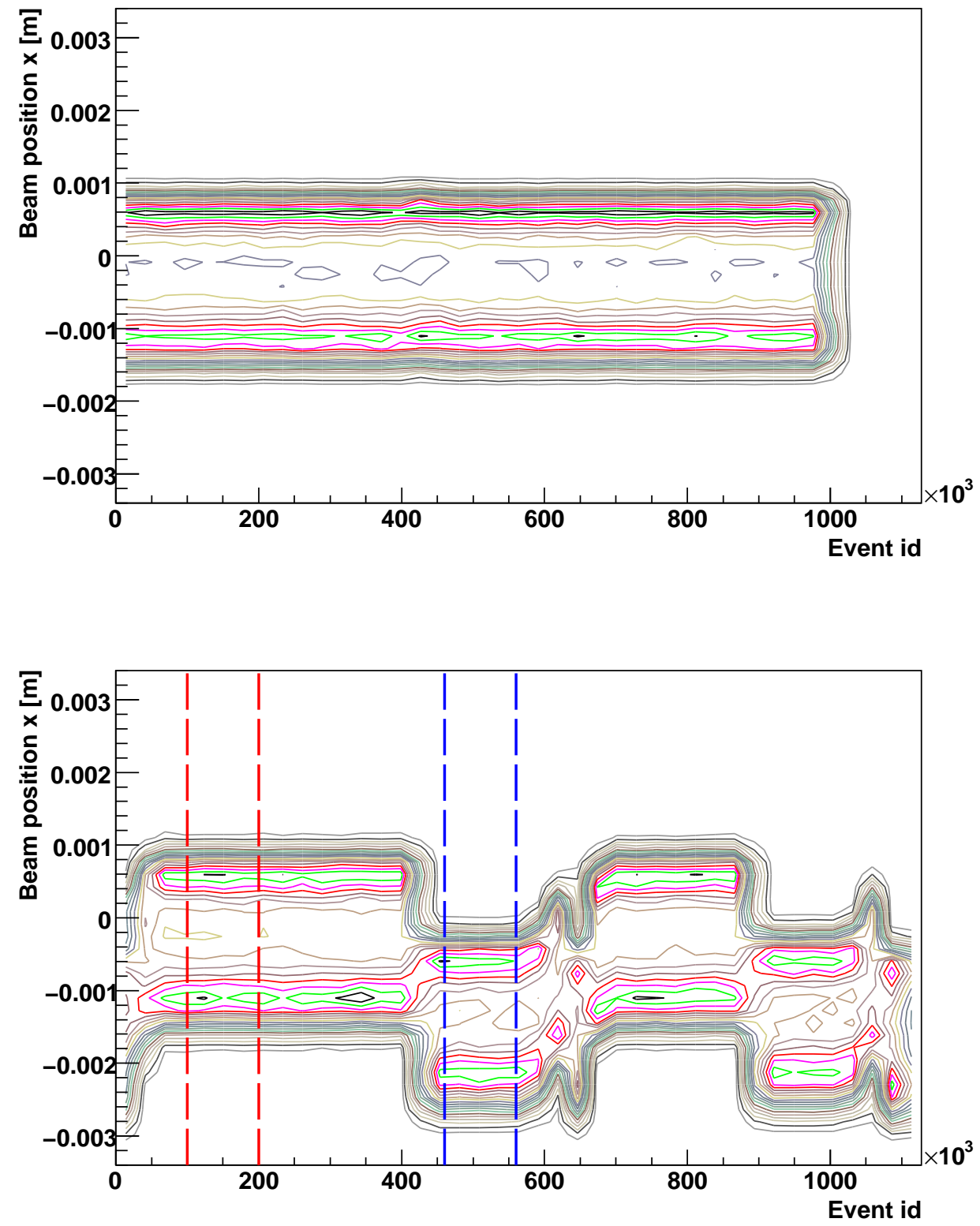

Figure 3.5: Contour plot of BPM x.

The $x$ axis is event id and the $y$ axis is $x_{\text {beam }}$. Beam positions of a unstable run are not uniform like the plot in bottom panel. 
is normalized for easy comparison. The blue curve deviates from the red one. In bottom plot, differences between two lines up to $2 \%$ are shown in the $Q^{2}$ distribution which is closely related with the cross section.

Fig. 3.7 shows the contour plot of $x_{\text {beam }}$ vs. event id after the BPM cut is applied to the bottom plot of Fig. 3.5. Beam trip regions are excluded and the cross section uncertainty from beam position is removed.

\subsubsection{Cherenkov sum cut}

In HRS, $\pi^{-}$also could be detected and they also generate events. The gas Chrenkov PID is used to eliminate $\pi^{-}$events from electrons. The momentum larger than 4.8 $\mathrm{GeV} / \mathrm{c}$ is required for $\pi^{-}$to make a Cherenkov light in this detector.

The gas Cherenkov detector has 10 PMTs and the Cherenkov sum cut is defined for the ADC sum from 10 PMTs to exceed 50. The efficiency of gas Cherenkov detector is known from other experiments and it is larger than 99.99\% [46].

The Cherenkov sum distribution of ${ }^{2} \mathrm{D}$ run is drawn in Fig. 3.8. The vertical red line is the criteria of Cherenkov sum, 50 .

The change of $W$ distribution after applying Cherenkov sum cut is shown in Fig. 3.9. The black line is before applying the cut and the red line is after applying the cut. This plot is from a run with the largest scattering angle. This run has the maximum $\pi^{-}$background and the contribution of $\pi^{-}$reaches only $\sim 2.5 \%$.

\subsubsection{Good event cut}

Only 'good events' are used in data analysis. Abandoned events are recovered in the yield calculation by introducing detector efficiencies instead. Considering properties and resolutions of detectors, the following cuts are applied to choose 'good' events.

- 'Good track cut' in trigger and VDC tracking.

The cut is defined with the following conditions. 

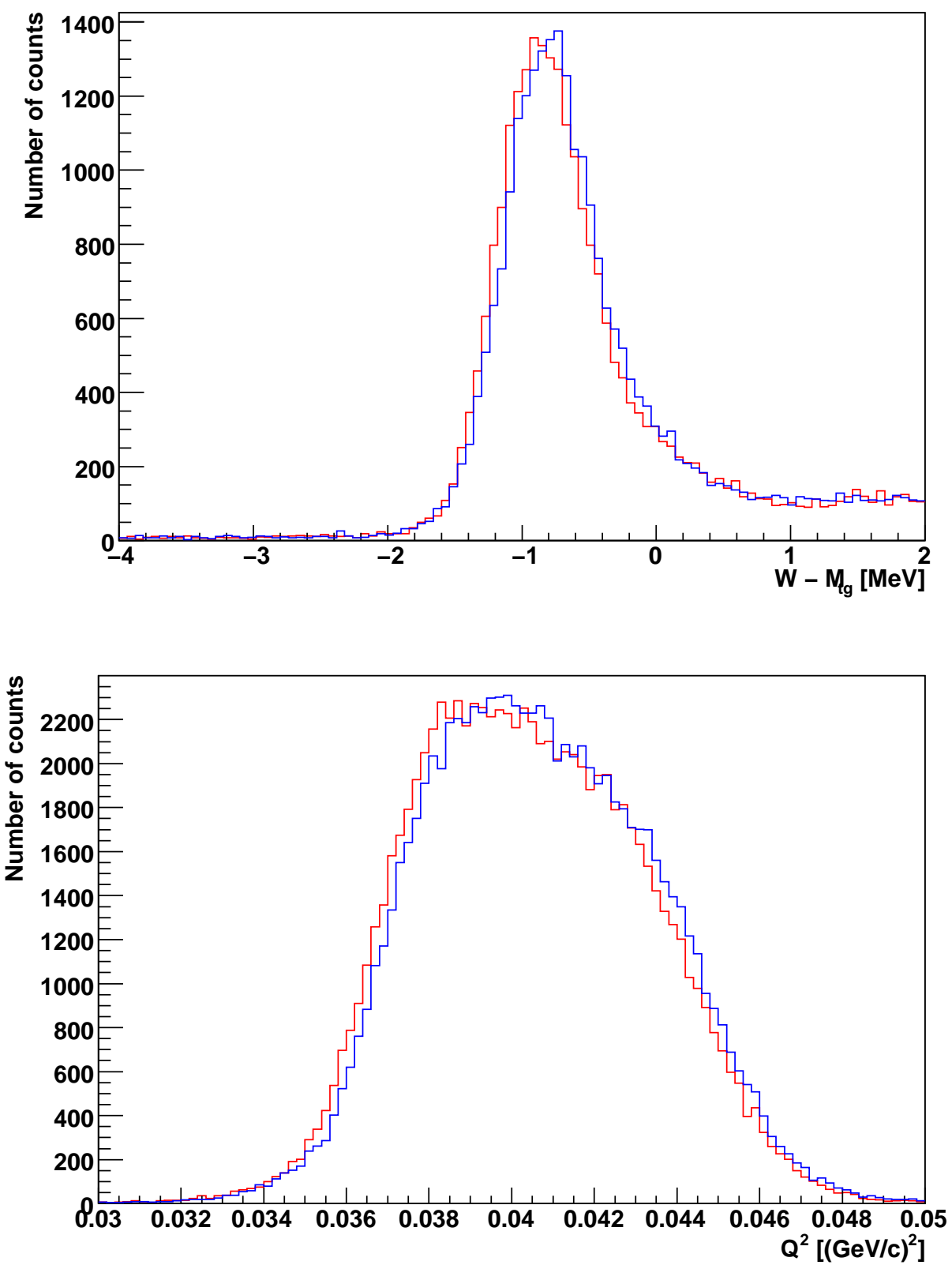

Figure 3.6: Distributions of $W$ and $Q^{2}$ from two different $x_{\text {beam }}$ regions in a run. The distribution of $Q^{2}$ shows maximum $2 \%$ difference and it is closely related with cross section. 


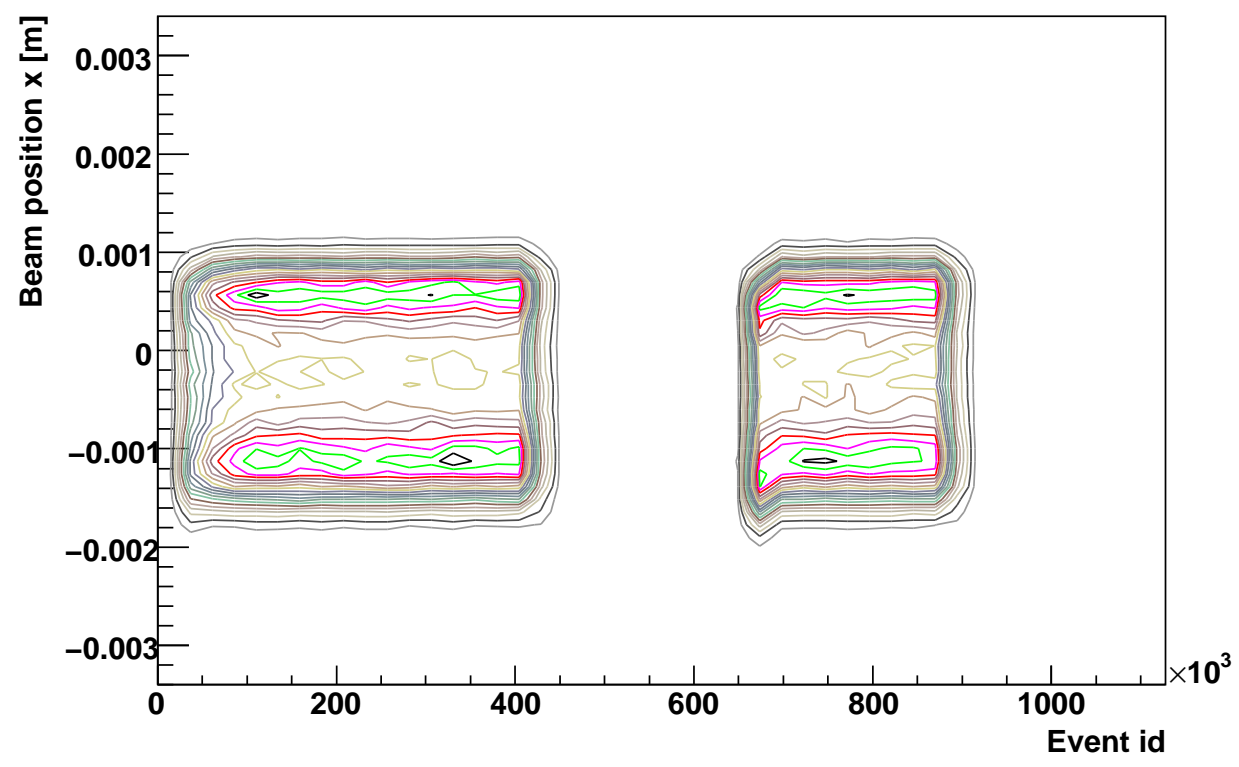

Figure 3.7: $x_{\text {beam }}$ becomes uniform distribution after BPM cut is applied.

- Event type 3(1) for left(right) arm

- An event should have one track

- Each wire plane has to contain one cluster

- Each cluster has the number of hit wires to be from 3 to 6

- 'Sieve cut' in collimator.

Events within square of $\pm 45 \mathrm{~mm} \times \pm 22 \mathrm{~mm}$ from the collimator center are chosen. Fig. 3.10 show two sieve cuts in sieve $x$ and sieve $y$ planes at collimator. Red vertical lines define the cuts.

- Reconstructed variables cut.

The momentum deviation $|\mathrm{dp}|<3.9 \%$ and $\mid$ tg-y $\mid<4 \mathrm{~cm}$.

The momentum acceptance of spectrometer is $\pm 4.5 \%$. However it is found from the white spectrum study that the actual acceptance was smaller than the expected value. Hence the tight cut of $\pm 3.9 \%$ is applied in yield calculation. 


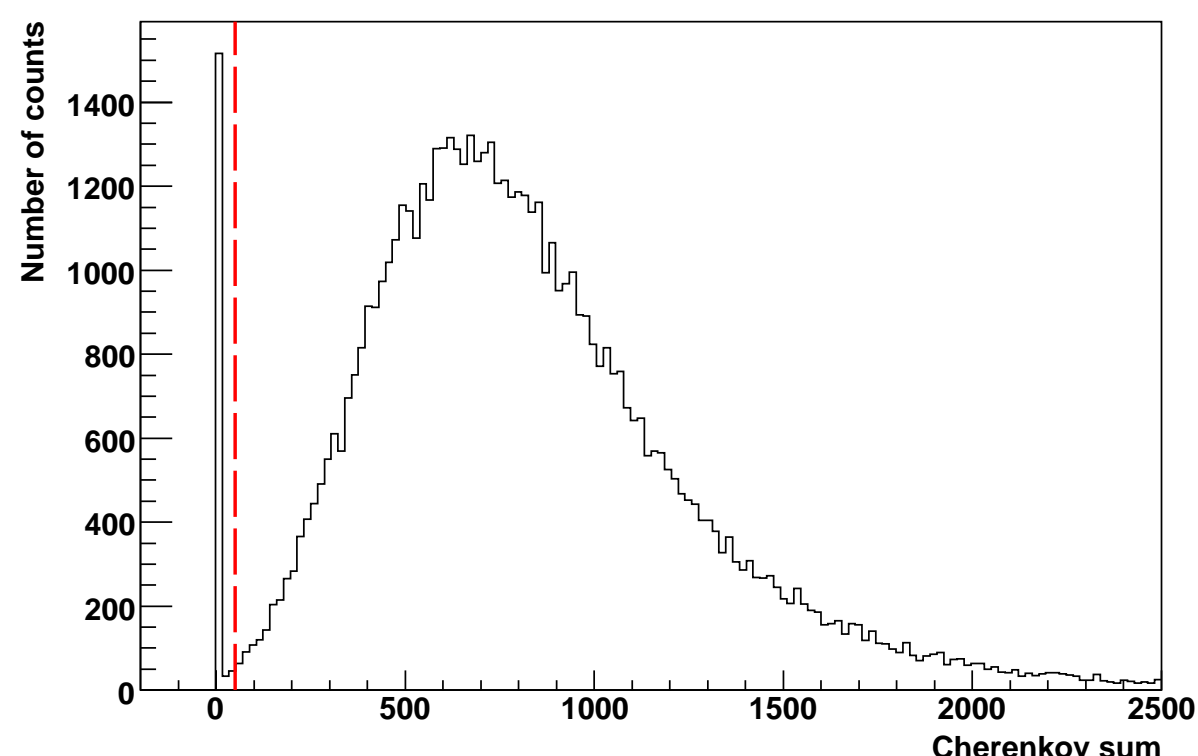

Figure 3.8: A distribution of Cherenkov sum. The vertical line is the criteria(50) for the Cherenkov sum cut.

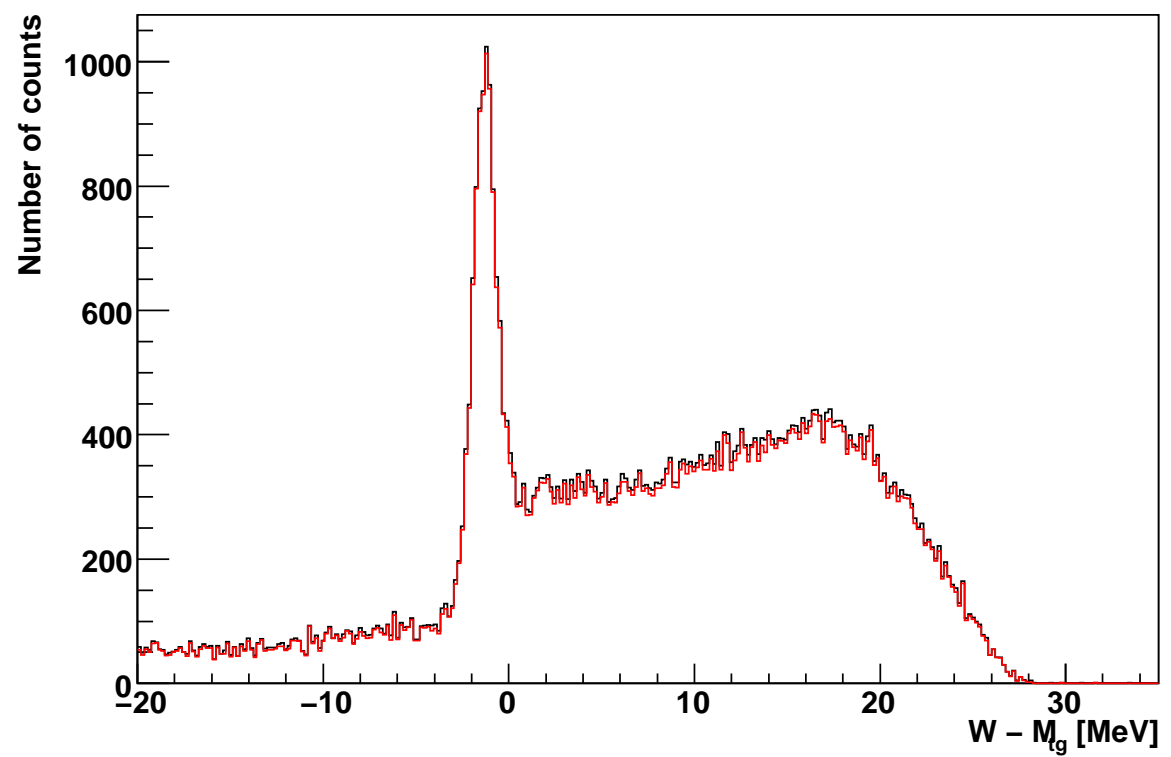

Figure 3.9: The $W$ distribution from a run with maximum $\pi^{-}$background. Black line : before $\pi^{-}$is removed. Red line : before $\pi^{-}$is removed. Totally $2.5 \%$ of events are removed. 

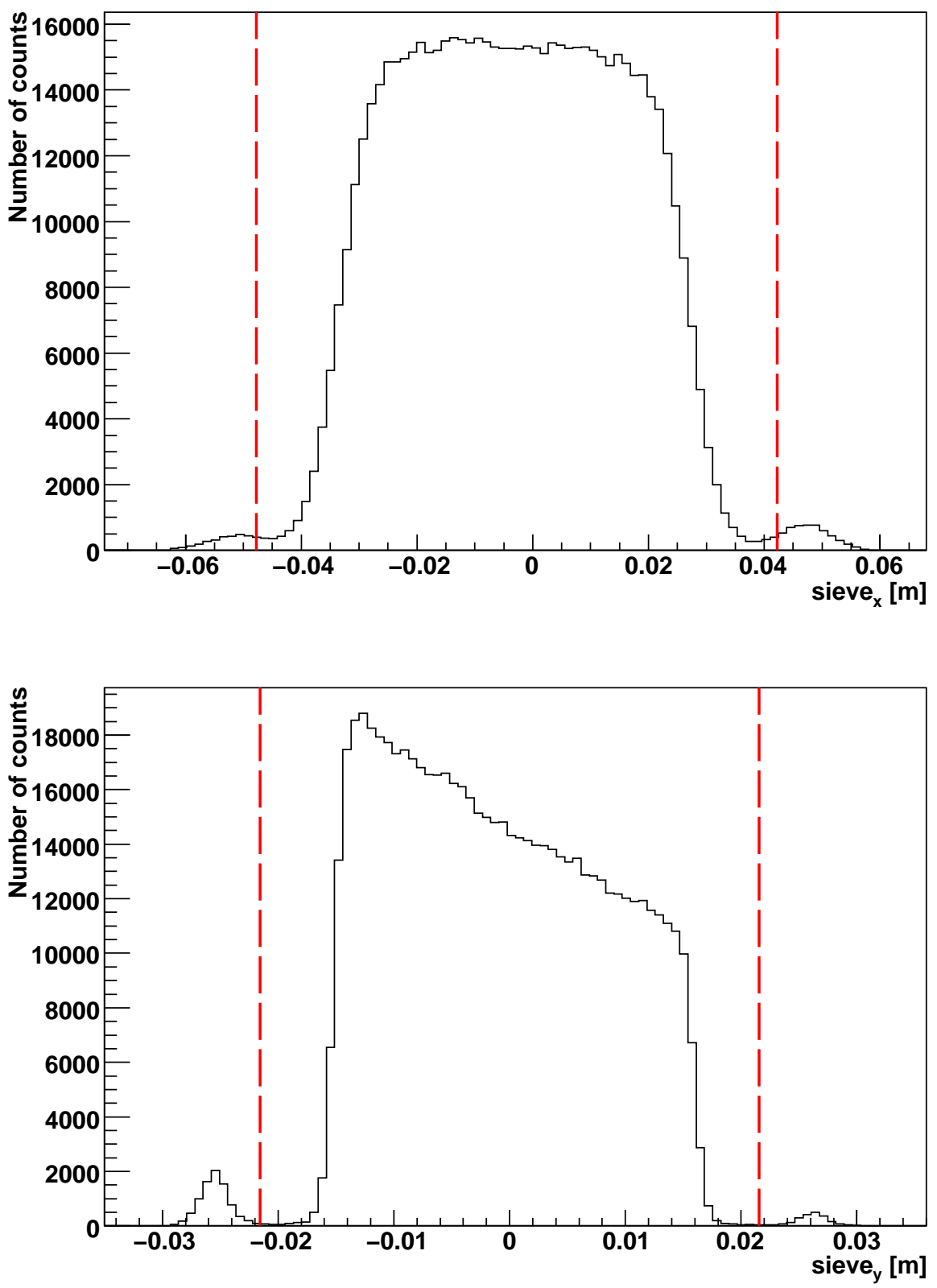

Figure 3.10: Sieve cut in the $x$ axis and $y$ axis in Transport coordinate system. Two pairs of vertical red lines define the cuts. 


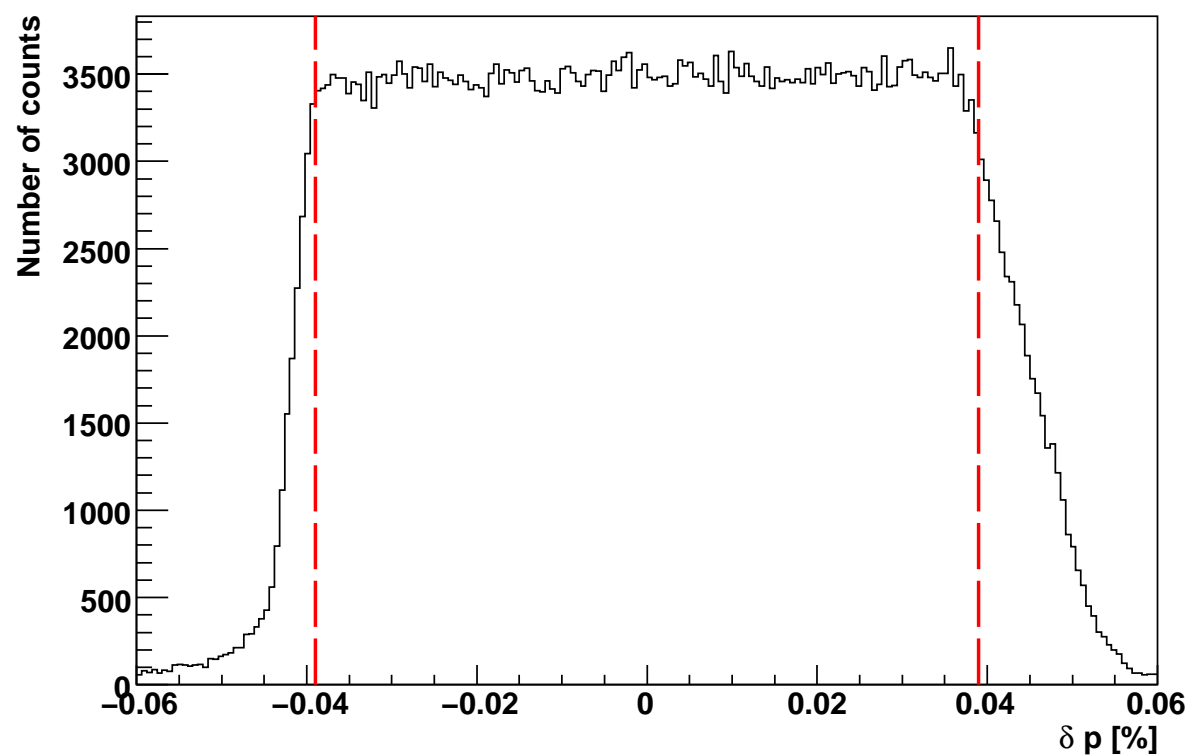

Figure 3.11: Momentum acceptance cut in a white spectrum. Two vertical red lines define the cut.

Fig. 3.11 shows the momentum distribution in a white spectrum run. Two vertical lines are placed at $\pm 3.9 \%$.

Further explanations about the previously mentioned variables are in following sections.

\subsection{Detector Efficiency}

In this section, several detector efficiencies are explained with the sequence of event handling procedure.

1. Two trigger scintillators open the gate and start the event process. $\rightarrow$

Trigger efficiency, $\varepsilon_{\text {trigger }}$.

2. Analyzer reconstructs event tracks in VDC wire. $\rightarrow$

Tracking efficiency, $\varepsilon_{\text {tracking }}$. 
3. Detected electrons are stored as events into hard disk. $\rightarrow$

the electronics dead time and computer dead time, $\mathrm{DT}_{e}$ and $\mathrm{DT}_{e}$.

\subsubsection{Trigger efficiency}

There are three planes of scintillators of S1, S0 and S2 for the left HRS, and two scintillator planes of S1 and S2 for the right HRS. They do the role of trigger for the VDC event tracking. Each particle coming into HRS is classified with specific event type by the predefined trigger logic. Definitions of important event types in this experiment are defined as follows.

- type1 (Right HRS) = Two or three coincidence signals among S1, S2 and gas Cherenkov

- type2 (Right HRS) = One signal between S1 and S2

- type3 (Left HRS) = Two or three coincidence signals among S1, S2 and gas Cherenkov

- type4 (Left HRS) = One signal between S1 and S2

If a normal scattered electron passes through the HRS detector alignment, they correspond to type3(1). On the other hand, events of type4(2) could be generated from non-electron particles with random directions(like cosmic rays) or scattered electrons with inefficient detector. In latter case, the event should be type 3(1) event originally but a scintillator failed to detect it. Trigger efficiency can be calculated by finding those events which are supposed to be type 3(1) by examining other

properties, but are classified with event type 4(2). Hence, the trigger efficiency is defined by Eq. 3.5.

$$
\begin{aligned}
\varepsilon_{\text {trigger }_{L}} & =\frac{N_{3}}{N_{3}+\operatorname{good} N_{4}} \\
\varepsilon_{\text {trigger }_{R}} & =\frac{N_{1}}{N_{1}+\operatorname{good} N_{2}},
\end{aligned}
$$


where $N_{3(1)}, N_{4(2)}$ are the numbers of event type3(1) and type4(2). $\operatorname{good} N_{4(2)}$ is the number of event type 4(2) but it was supposed to be event type3(1).

Fig. 3.12 shows the change of momentum distribution in a cosmic run after good track cut which is explained in section 3.3.2 is applied. Only $1 \%$ of cosmic events are survived. Because the total number of cosmic events is negligible in the kinematics of this experiment, the good track cut is adoptable to find $\operatorname{good} N_{4(2)}$. So $\operatorname{good} N_{4(2)}$ is the number of events with type 4(2) satisfying tracking cut.

$\varepsilon_{\text {trigger }}$ is obtaind for all runs and the efficiency is at least $99.7 \%$.

\subsubsection{Tracking efficiency}

VDCs detect tracks of scattered electrons and reconstruct target variables which give the scattering information. There exist two cases for the tracking failure. One is the inefficiency of VDC wire and another is the failure of track finding(track reconstruction algorithm). The efficiency of a wire can be found by counting events that the wire did not fire but two neighboring wires did fire. In an ordinary environment, the trigger rate is smaller than several tens of $\mathrm{kHz}$ and the wire efficiency is $\gtrsim$ $99.9 \%$. The wire efficiency cannot be separated from the tracking efficiency and it is incorporated into the multi-track correction in runs with extraordinary high trigger rate. Further explanations are in section 4.4. Hence wire efficiency is ignored and the failure of track finding is solely considered for the tracking efficiency.

When Analyzer reconstructs VDC tracks, the implemented algorithm could fail to obtain correct tracks. Sometimes unnecessary events such as delta rays and cosmic rays corrupt ordinary VDC tracks. The good track cut is required to erase such corruption and it selects well reconstructed events only. [36]

An event that passed the cut are mentioned as 'good track event' from here. It is assumed that all events of type 3(1) for the left(right) arm have only one electron and events not satisfying the good track cut are generated by the failure of tracking algorithm. The good track cut is already defined in section 3.2.5. 

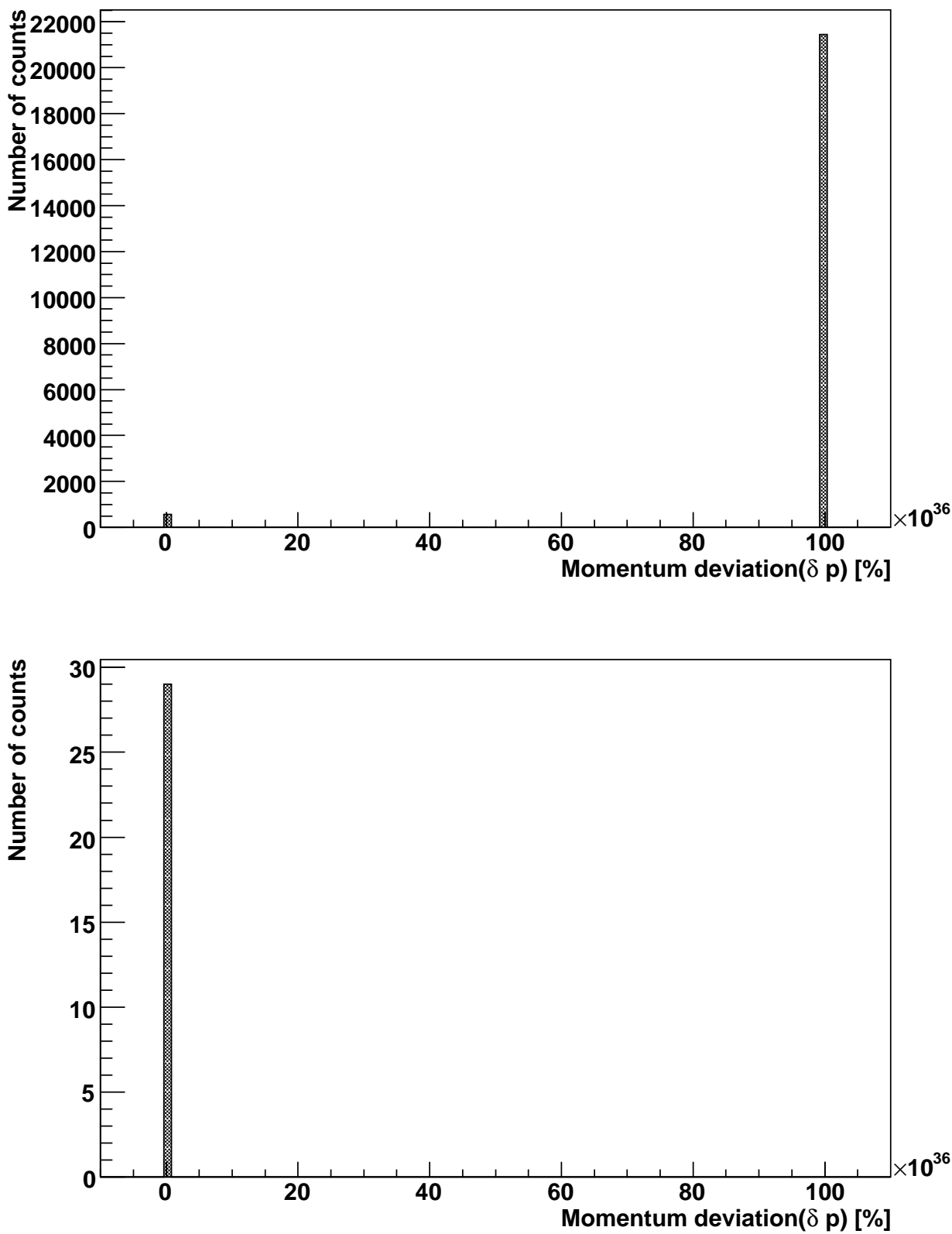

Figure 3.12: Momentum distributions from a cosmic run. The bottom plot is made from the top plot after the good track cut is applied. Most cosmic events are removed by tracking cut. 
Electrons excluded by the good track cut are compensated by the tracking efficiency which is given by Eq. 3.7.

$$
\varepsilon_{\text {track }}=\frac{N_{\text {good track }}}{N_{\text {type3(1) }}-N_{\text {cosmic }}}
$$

where $N_{\text {type3(1) }}$ is the number of events of event type $3(1), N_{\text {good track }}$ is the number of events satisfying good track cut, $N_{\text {cosmic }}$ is the number of events in the momentum setting satisfying the good track cut and Cherenkov sum cut. And $N_{\text {cosmic }}$ is scaled by the time of each run. Analysis shows that $N_{\text {cosmic }}$ is negligible in the kinematic setting of this experiment.

Trigger rates of liquid target runs at some kinematics exceeds $100 \mathrm{kHz}$. In such cases, number of multi-track events increase and they cannot be neglected. Fig. 3.13 shows two distributions of number of tracks from a high rate run $(128 \mathrm{kHz})$ and a normal $\operatorname{run}(0.3 \mathrm{kHz})$ in top and bottom panel. The $x$ axis is the number of tracks and the $y$ axis is the number of counts. Both of them are made from ${ }^{2} \mathrm{D}$ target and normalized to one million events for comparison. While the contribution of multi track is less than a few $\%$ in bottom panel, it exceeds $15 \%$ in top panel. The assumption that a good track event has only one electron should be modified in such high rate runs. Because several electrons enter into spectrometer simultaneously, they can make a multi-track event with several electrons. Further explanations about the method to correct the multi-track contribution in high rate are in section 4.4 .

\subsubsection{Dead time}

Two dead time of electronics $\mathrm{DT}_{e}$ and computer $\mathrm{DT}_{c}$ are considered for the efficiency calculation.

The total dead time efficiency is given by Eq. 3.8.

$$
\varepsilon_{\mathrm{DT}}=\left(1-\mathrm{DT}_{e}\right) \cdot\left(1-\mathrm{DT}_{c}\right)
$$



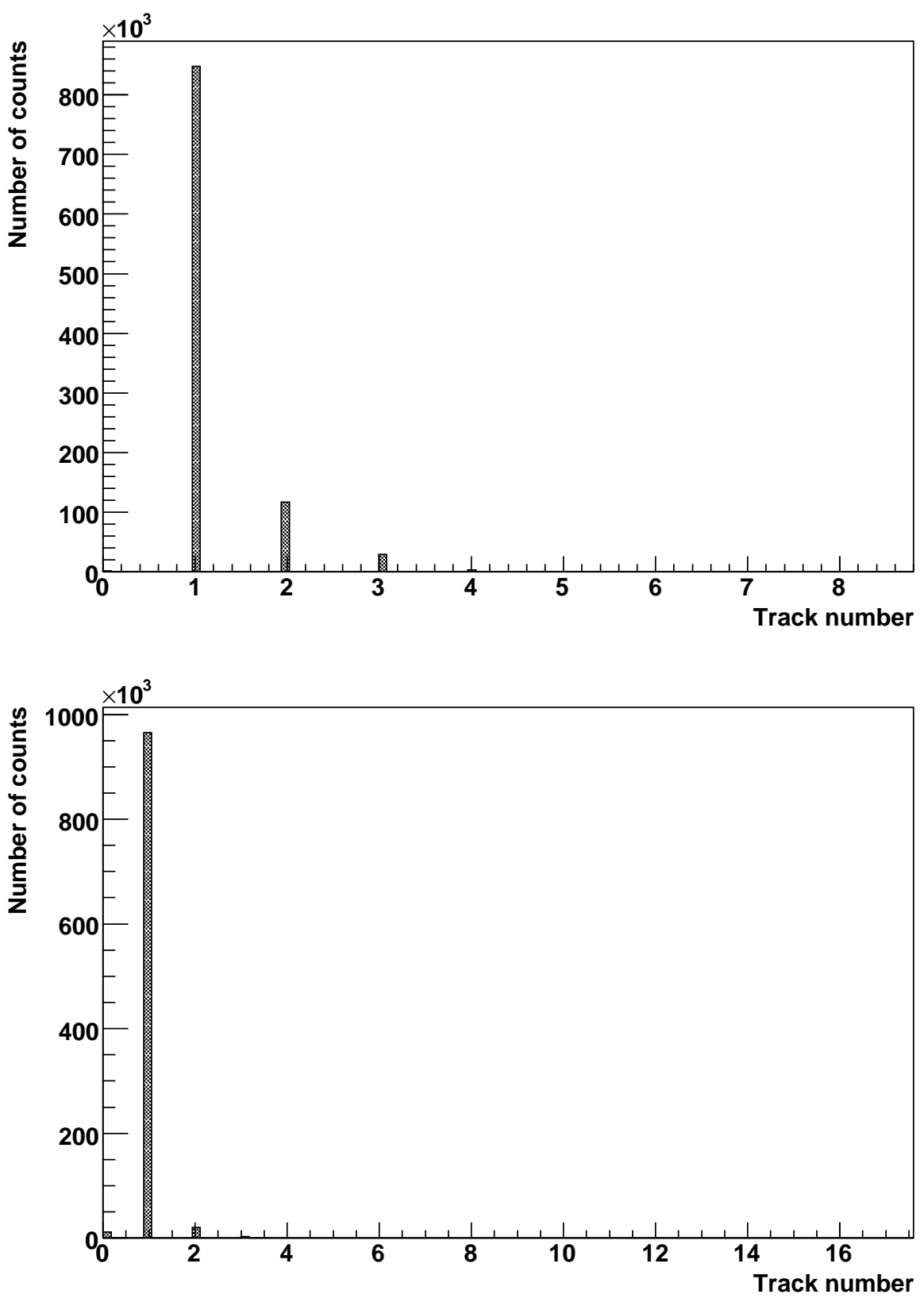

Figure 3.13: Distributions of number of tracks. The top panel is from a high rate $\operatorname{run}(128 \mathrm{kHz})$ and the bottom panel is from an ordinary run $(0.3 \mathrm{kHz})$. Multi-track events occupy $15 \%$ of total events in top panel. 


\section{Electronics dead time}

In an electronics with dead time $\tau$, the efficiency is given by Eq. 3.9. [47]

$$
\varepsilon=1-P(t)=1-R \int_{r}^{\infty} \exp (-R t) d t=1-\exp (-R \tau)
$$

where $R$ is the count rate of trigger.

For Hall A DAQ system, it is known that $\tau \approx 100 \times 10^{-9}$ [48] .

Hence the electronics dead time can be calculated by Eq. 3.10.

$$
\mathrm{DT}_{e}=\exp \left(-R \cdot 100 \times 10^{-9}\right)
$$

At a kinematics with the highest triggger $\operatorname{rate}(200 \mathrm{kHz})$, the dead time reaches approximately $2 \%$. But the trigger rate is smaller than $10 \mathrm{kHz}$ in most runs and the dead time effects in such runs are negligible.

\section{Computer dead time}

The computer dead time occurs during the process of writing experimental data into computer hard disk. DAQ system stores each event by its type $i$ separately and controls the recording rate of type $i$ by the prescale factor $\mathrm{ps}_{i}$. So the computer dead time is defined for each event type $i$.

When a prescale factor $\mathrm{ps}_{i}$ is given, DAQ records only $\mathrm{ps}_{i}$ th events from all entering events of type $i$. During the experiment, $\mathrm{ps}_{3,1}$ are set to maintain the dead time smaller than $10 \%$.

The computer dead time can be calculated from the ratio of lost events in recording

to the triggered number. The efficiency is written by Eq. 3.11 for each event type $i$.

$$
\varepsilon_{\mathrm{DT} c i}=1-\mathrm{DT} c i=\frac{\mathrm{PS}_{i} \times N_{\mathrm{DAQ} i}}{N_{\text {trigger } i}}
$$


where $N_{\mathrm{DAQ} i}$ is the number of recorded events in DAQ and $N_{\text {trigger }}$ is the triggered number with type $i$.

Efficiencies $\varepsilon_{\mathrm{DT} 3(1)}$ and $\varepsilon_{\mathrm{DT} 4(2)}$ are calculated for all runs. $\varepsilon_{\mathrm{DT} 3(1)}$ is used for the dead time efficiency and $\varepsilon_{\mathrm{DT} 4(2)}$ is used for the trigger efficiency.

\subsection{Coordinate System}

Kinematic variables describing beam electrons and scattered electrons are defined in three coordinate systems. These systems are explained in this section.

\subsubsection{Laboratory coordinate system}

The Laboratory coordinate(or Lab coordinate) system has its origin at the Hall center. The $\mathrm{z}$ axis is along the beam direction. And the $x$ axis is toward the left side of beam direction in the horizontal plane and perpendicular to $\hat{z}$. Fig. 3.4.1 shows the Lab. coordinate system seen from the sky.

This is the standard reference for other coordinate systems. Several variables such like scattering angle, beam positions, scattering reaction positions, target positions and mispointing of HRS are defined in this system.

\subsubsection{Target coordinate system}

While positions of beam and HRS are described in the Lab coordinate system, all dynamical variables of scattered electrons are defined in the Target coordinate system. The origin is defined with the target center which should be the same with the Hall center in ideal case. The $\mathrm{z}$ axis points towards the spectrometer center line and the $x$ axis points vertically downward.

The trajectory and momentum of a scattered electron are expressed by a vector of Eq. 3.12. It can be reconstructed from a vector at the focal plane where electrons are detected. 


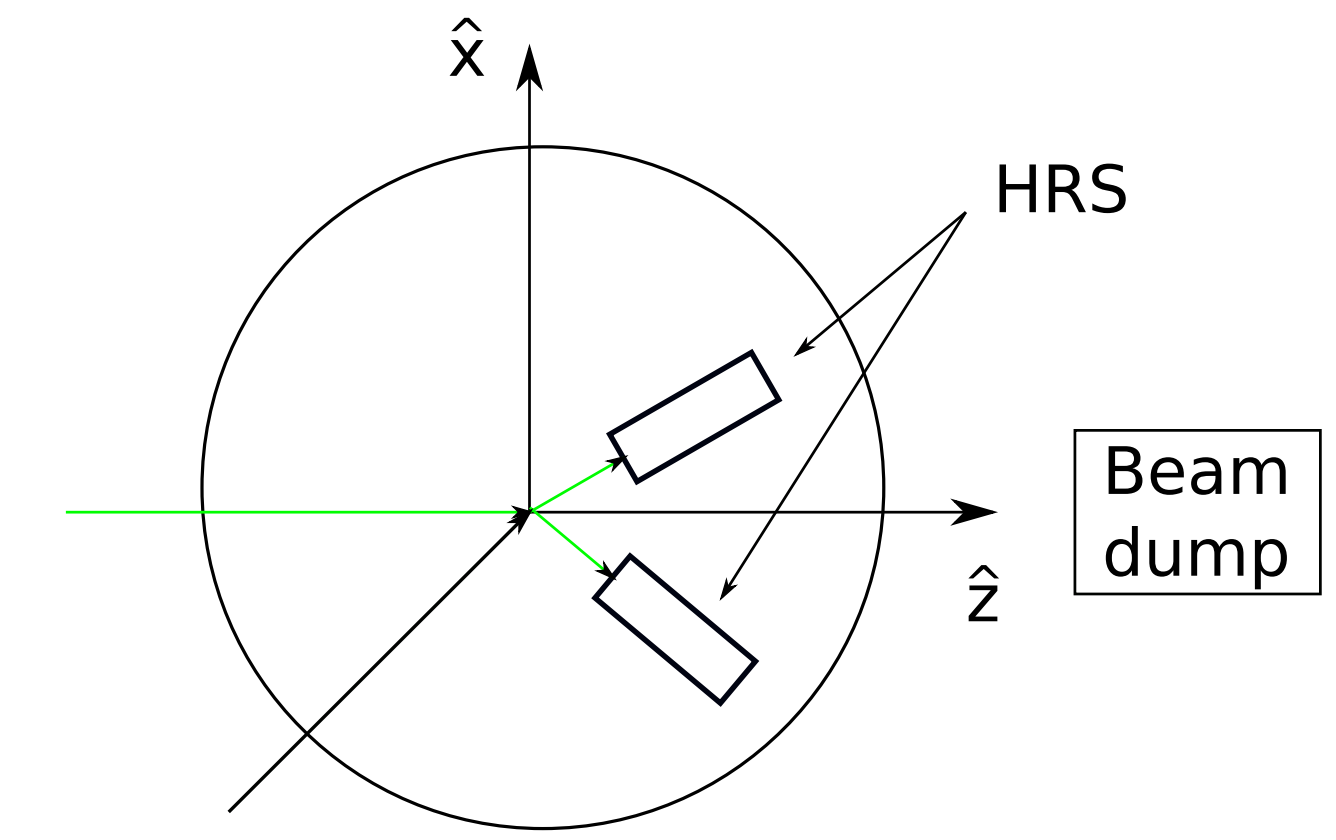

\section{Hall center}

Figure 3.14: The Laboratory coordinate system is seen from the sky.

$$
\vec{x}_{\mathrm{tg}}=\left[\begin{array}{c}
x_{\mathrm{tg}} \\
\theta_{\mathrm{tg}} \\
y_{\mathrm{tg}} \\
\phi_{\mathrm{tg}} \\
l \\
\delta
\end{array}\right],
$$

where $x_{\operatorname{tg}}$ and $\theta_{\operatorname{tg}}$ are the displacement and tangent of angle in the dispersive plane, $y_{\operatorname{tg}}$ and $\phi_{\operatorname{tg}}$ are the displacement and tangent of angle in the transverse plane, $l$ is the path length difference between the electron trajectory and reference trajectory (center line) and $\delta$ is momentum deviation from the central momentum setting in percentage.

The blue line in Fig. 3.15 are the axes of Target coordinate system. 


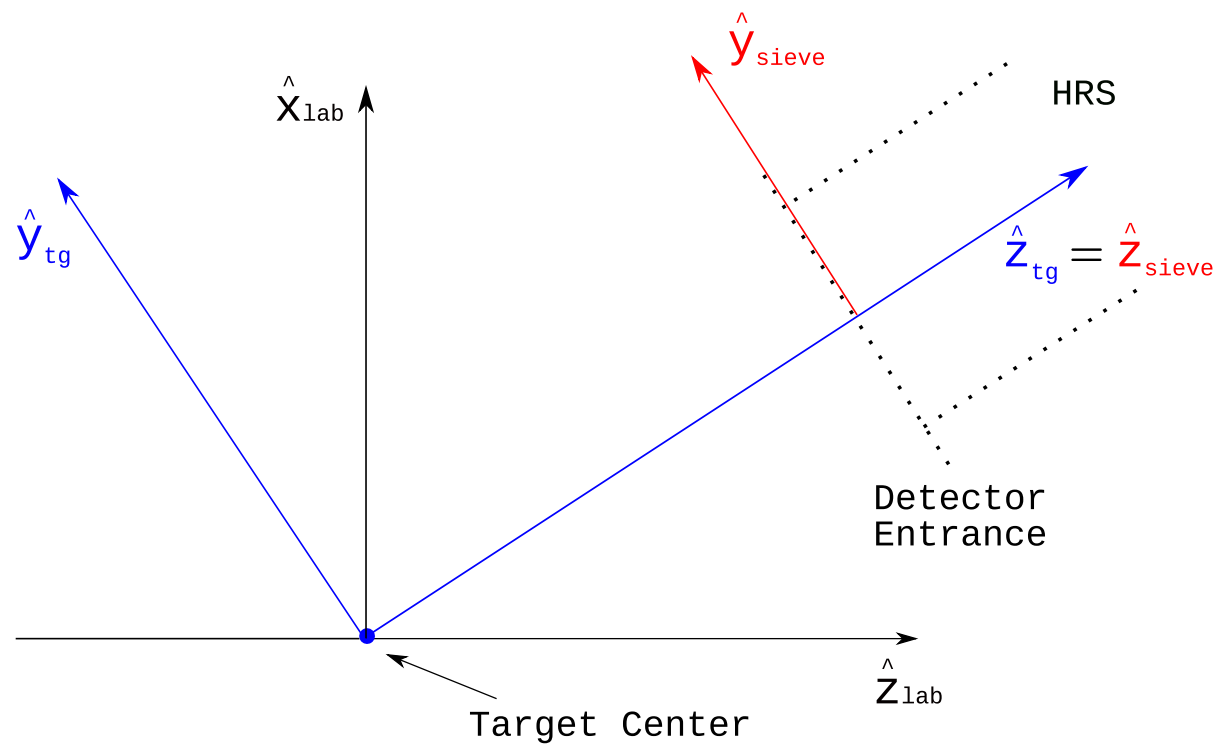

Figure 3.15: Target coordinate system(blue) and Transport coordinate system at the detector entrance(red).

\subsubsection{Transport coordinate system}

The Transport coordinate system is defined along the reference trajectory of scattered electron. The origin is the reference trajectory and this system is the same with Target coordinate system except the origin. Especially, the Transport coordinates at the center of detector entrance define the solid angle.

The axes are drawn in Fig. 3.15 with red line.

\subsection{Calibrations of target variables}

\subsubsection{Variables of beam and scattered electrons}

There are several variables to describe beam and scattered electrons.

- $x_{\text {beam }}, z_{\text {beam }}, z_{\text {react }}$ : Location of ${ }^{2} \mathrm{D}\left(e, e^{\prime}\right)^{2} \mathrm{D}$ scattering in the Lab coordinate system. 
$x_{\text {beam }}$ and $y_{\text {beam }}$ are positions of beam and $z_{\text {react }}$ is crossed point between two trajectories of beam and scattered electron which is given as ${ }^{2}$

$$
z_{\text {react }}=-\left(y_{\mathrm{tg}}+D\right) \frac{\cos \left(\arctan \phi_{t g}\right)}{\sin \left(\theta_{0}+\arctan \phi_{t g}\right)}+x_{\text {beam }} \cot \left(\theta_{0}+\arctan \phi_{t g}\right)
$$

where $\mathrm{D}$ is the value of horizontal HRS mispointing and $\theta_{0}$ is the reference scattering angle.

- $y_{\mathrm{tg}}, \phi_{\mathrm{tg}}, \theta_{\mathrm{tg}}, \delta\left(=d p_{\mathrm{tg}}\right)$ : Target variables describing a scattered electron.

Each of them is an element of $\vec{x}_{\mathrm{tg}}$ in Eq. 3.12. $d p_{\mathrm{tg}}$ is the momentum deviation from the central momentum, $\frac{p-p_{\text {central }}}{p_{\text {central }}}$.

Scattering angle of each electron is given by Eq. 3.14.

$$
\theta_{\text {scat }}=\arccos \left(\frac{\cos \left(\theta_{0}\right)}{\sqrt{1+\theta_{t g}^{2}+\phi_{t g}^{2}}}\right)
$$

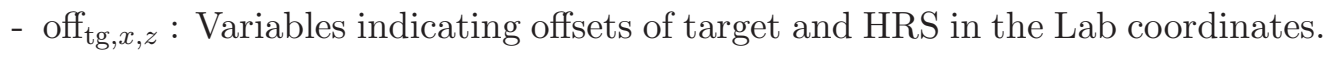
off $_{\text {tg }}$ is the target offset in $\hat{z}$ direction. off $x, z$ are the components of spectrometer axis displacement and they are known as HRS mispointing.

- $x_{\text {sieve }}, y_{\text {sieve }}, z_{\text {sieve }}(=L)$ : The location of scattered electron at the detector entrance in the Transport coordinate system.

$x_{\text {sieve }}$ and $y_{\text {sieve }}$ define the solid angle of kinematic setting. $L$ is the distance between the Hall center and detector entrance center.

$x_{\text {sieve }}$ and $y_{\text {sieve }}$ are related with target variables by Eq. 3.15

$$
\begin{aligned}
& y_{\text {sieve }}=y_{\mathrm{tg}}+L \phi_{\mathrm{tg}} \\
& x_{\text {sieve }}=x_{\mathrm{tg}}+L \theta_{\mathrm{tg}}
\end{aligned}
$$

\footnotetext{
${ }^{2} z_{\text {react }}$ is the same with the target center for the thin foil target and it extends to any point between two container walls for the liquid target.
} 


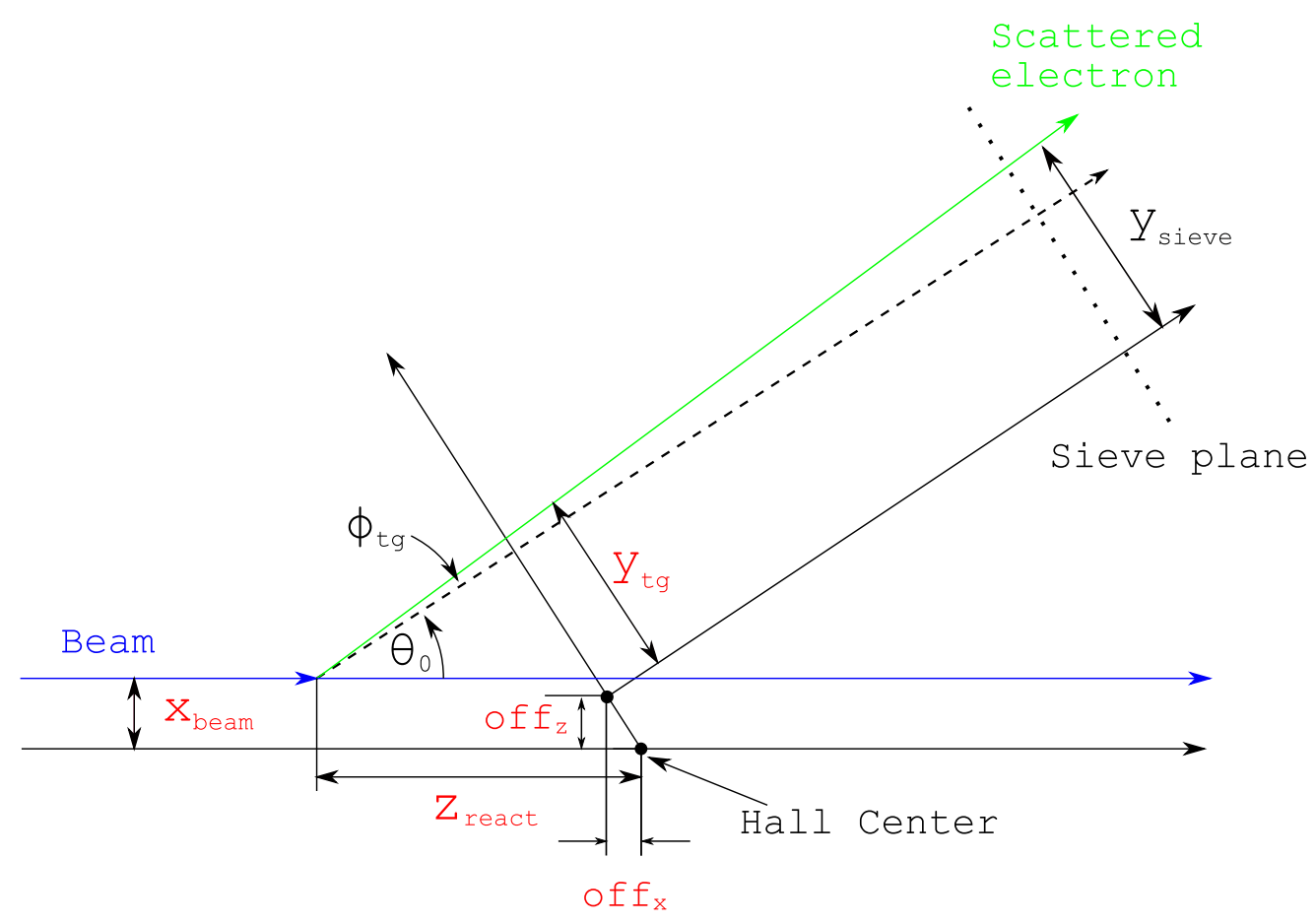

Figure 3.16: Target variables

Fig. 3.16 shows some of these variables and their relations. Variables off $x, z, z_{\text {react }}$ and $x_{\text {beam }}$ are closely correlated and give similar effects to $y_{t g}$.

\subsubsection{Change of target variables}

Scattered electrons can be deviated from the reference trajectory by several reasons. Three factors of HRS mispointing, extended target and rastered beam are considered in this analysis. Target variables of scattered electrons are defined in the Target coordinate system with the axis of spectrometer center as $\hat{z}$ direction. But the axis line could be deviated from the Hall center during the experiment and it is known as HRS mispointing. When the spectrometer is moved for a new angle setting, the values of mispointing also change. Then the detector entrance is moved from the original location and the acceptance region is changed. Hence target variables of 
accepted events are modified.

While the HRS mispointing gives an indirect effect with the change of acceptance region, two other factors generate the direct change of target variables. First, there are effects generated from target types. Two liquid targets of ${ }^{1} \mathrm{H}$ and ${ }^{2} \mathrm{D}$ are contained in an aluminum cell with $4 \mathrm{~cm}$ length. The scattering interaction can occur in any location between the two walls. In such case, the scattering angle increases with $z_{\text {react }}$.

Second, the raster is turned on during this experiment and it changes target variables. It maintains the beam size with $3 \times 3 \mathrm{~mm}^{2}$. If the beam position $x$ in Lab. coordinates increases from the target center, the scattering angle could become smaller. Variations of reaction point and beam position can change target variables directly. Spectrometers detect scattered electrons at the focal plane. Focal plane variables are related with target variables by a transporting matrix $T$ as

$$
x_{\mathrm{tg} i}=\tilde{T}_{i j} x_{\mathrm{fp} j} .
$$

If the previous two factors are considered, target variables can be changed even though the HRS detecting location(focal plane variables, $\overrightarrow{x_{\mathrm{fp}}}$ ) is the same. Changes of target variables can be calculated as

$$
\begin{gathered}
x_{\mathrm{tg} i}+\delta x_{\mathrm{tg} i}=\tilde{T}_{i j} x_{\mathrm{fp} j} \\
\delta x_{\mathrm{tg} i} \tilde{T}_{i j}^{-1}=0
\end{gathered}
$$

where $x_{\operatorname{tg} i}+\delta x_{\operatorname{tg} i}$ is the new target variable after calibration. Further explanations are in following sections.

\subsubsection{HRS mispointing}

Fig. 3.16 already showed that mispointing variables, off $x, z$, are correlated with $z_{\text {react }}$

and $x_{\text {beam }}$. In the plot, the HRS mispointing can be calculated by target variables 
and it is given by Eq. 3.19.

$$
\begin{aligned}
D & =-y_{t g}-x_{\text {beam }} \cdot \cos \theta_{0}+z_{\text {react }} \cdot \sin \theta_{0} \\
\text { off }_{x} & =D \cos \theta_{0} \\
\text { off }_{x} & =-D \sin \theta_{0}
\end{aligned}
$$

where off $y$ is the vertical component of mispointing and is related with $\theta_{\operatorname{tg}}$. Because the cross section is insensitive to $\theta_{\mathrm{tg}}$, off $y$ is ignored here.

Especially in thin foil target like carbon, $z_{\text {react }}$ is the same with the fixed target position and Eq. 3.19 can be rewritten by Eq. 3.22.

$$
D=-\bar{y}_{t g}-\bar{x}_{\text {beam }} \cdot \cos \theta_{0}+\text { off }_{\text {target }} \cdot \sin \theta_{0}
$$

where $\bar{y}_{t g}$ and $\bar{x}_{\text {beam }}$ indicate average values.

Values of mispointing are obtained for each kinematics by Eq. 3.22. The calculation used carbon runs except two kinematics which have very few events. For exceptional kinematics, the front wall of dummy target has been used.

\subsubsection{Raster calibration and extended target}

The raster calibration is applied with two procedure.

- First, the rastered beam position of each event is obtained.

- Second, corrected kinematic variables are calculated from the new reaction point and beam position.

The relation between the beam position and reaction point are known from Eq. 3.13. So $z_{\text {react }}$ of each event can be calculated if the beam position is measured. The beam position in Hall A is measured by two BPMs. They measure positions at two

directions, $\hat{x}$ and $\hat{y}$ in the Lab. coordinates system. The positions at the target are 

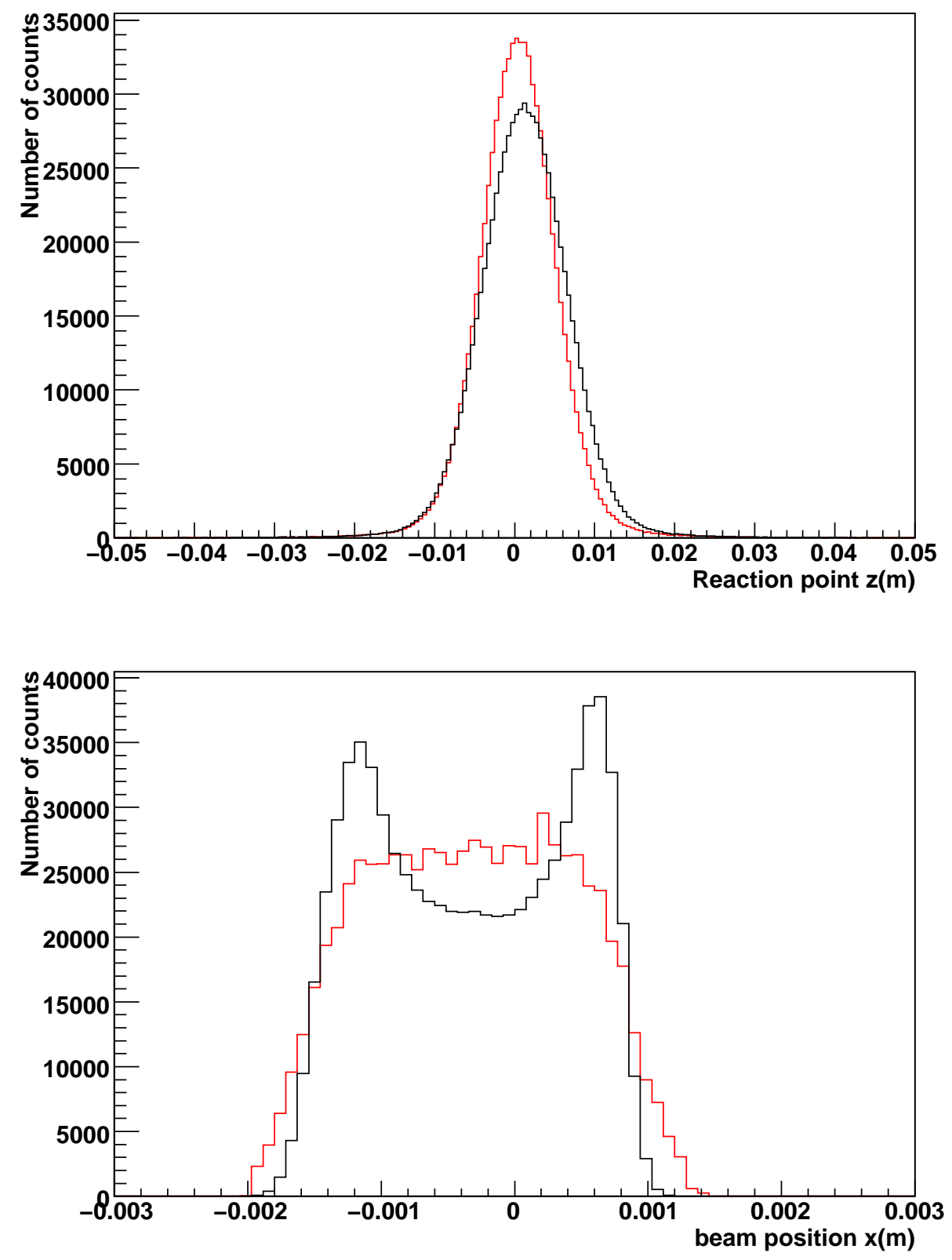

Figure 3.17: The change of variables $\left(z_{\text {react }}\right.$ and beam position $\left.\mathrm{x}\right)$ after calibration. Black line : before calibration ; Red line : after calibration 

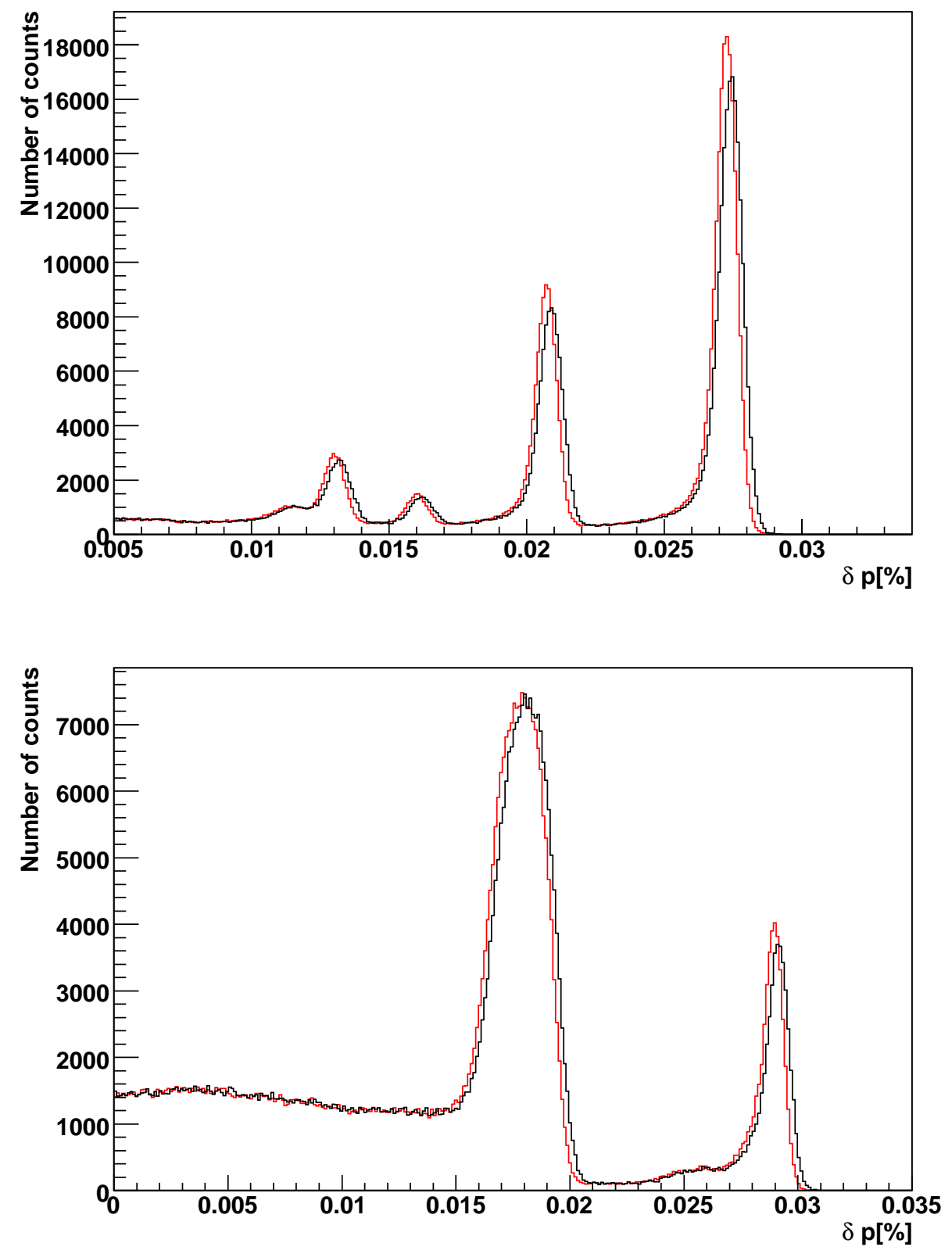

Figure 3.18: The change of $d p_{\text {tg }}$ after calibration for ${ }^{12} \mathrm{C}(\mathrm{Top})$ and ${ }^{2} \mathrm{D}$ (Bottom). Black line : before calibration ; Red line : after calibration 
extrapolated from the positions at two BPMs. While BPMs give the good average position for some period, they are not fast enough to detect the position of each event. It means BPMs cannot be used for rastered beam though they are good for the unrastered beam. Instead, the raw currents of raster, which change positions of raster, can give the position information of each event. This job is known as the raster calibration. [41]

The raster raw current gives the relative information and the value is proportional to the position deviation from the beam center(average position). So the raster raw current is compared with the average value of BPMs to find the absolute average position first and then the linear equation between the raw current and real position is extracted from the relative position. This method is only valid when the beam is stable and no abrupt change of beam position happens since the raw current give the relative information alone. Sometimes the beam is unstable during experiment. Hence the BPM cut in section 3.2.3 is introduced to select stable beam regions.

If the new beam position is obtained, change of target variables should be reconsidered. This is implemented in Analyzer. After the rastered beam position is obtained, the input option of beam position should be replaced with the rastered beam. Then root files are newly replayed and calibrated target variables are obtained.

Fig. 3.17 and Fig. 3.18 show the change of some variables after calibration. The black line is before calibration and the red line is after calibration. The top panel in Fig. 3.17 shows distributions of $z_{\text {react }}$ from a carbon run. The distribution of $z_{\text {react }}$ became sharper after calibration. The bottom panel shows the change of beam position $\mathrm{x}$. The black line is the histogram of BPMs and the red line is the calibrated rasterd beam position.

Fig. 3.18 shows the distribution change of $d p_{\text {tg }}$ after calibration. The top panel is for a carbon run and the bottom panel is for a ${ }^{2} \mathrm{D}$ run. Distributions are slightly changed after calibration. Calibrated curve is sharper in the carbon distribution, but it is similar in ${ }^{2} \mathrm{D}$ runs because the extended target effect(reaction point) is 
larger than the raster effect in the extended target. 



\section{Chapter 4}

\section{Data Analysis Part II : Normalization and Yield Extraction}

\subsection{Beam Current Calibration}

\subsubsection{Low beam current}

Beam current, $I$, is monitored by the Hall A BCM system during experiment. The BCM of Hall A consists of two RF cavities, Unser monitor, associtiated electronics and a DAQ system. In addition, a OLO2 cavity monitor and a Faraday cup at the injector of accelerator are used to provide the information of absolute beam currents during calibratoin runs.

The Unser monitor is sandwitched by two RF cavities along the beam passage. The front cavity is called as upstream BCM and the back one is called as downstream BCM. Each BCM gives a voltage output value proportional to the beam current and the electronic signal is divided into three channels. One channel is preserved and the other two channels are amplified by factors of 3 and 10 times. Hence overall 6 BCM 
data exist: BCM u1, u3, u10, d1, d3 and d10. Alphabets u and d refer the upstream and downstream and following numbers refer the amplification number. The BCM d1 have had relatively small variation and noise during this experiment so it is chosen for this analysis. These BCM values are measured during the experiment instead of beam current. Beam currents during experiment runs are obtained by following procedure.

1. Calibration runs are operated with several known beam current values.

2. BCM voltage values are also obtained in the calibation runs.

3. The linear equation between the BCM d1 and beam current $I$ is extracted.

4. The equation is applied to the BCM d1 value of every run and the beam current $I$ is found at each run.

The OLO2 monitor is used to measure the absolute beam current in the calibration run. It is known that the monitor is valid in the region of $I \geq 10 \mu A$. It gives bigger uncertainties reaching several $\%$ in $I<10 \mu A$ with decreasing beam current. At some low angle kinematics, the trigger rate of liquid target is very high and the beam current is kept below $10 \mu A$ to reduce the event rate.

Table 4.1 shows the beam current over the angle setting for liquid targets. Beam currents of most kinematics are smaller than $10 \mu \mathrm{A}$ and the minimum current is 2 $\mu A$. Hence a new calibration method is required for runs with low beam current and the silver calorimeter is adopted to resolve this.

For the BCM calibration of this experiment, both of the OLO2 monitor and silver calorimeter are used. Especially the silver calorimeter gives the information of low beam current whereas OLO2 gives that of high beam current.

Absolute beam current values from calibration runs are tabulated in Table $4.2^{1}$ together with BCM d1 values.

\footnotetext{
${ }^{1}$ There are more data points of silver calorimeter. But the minimum current during the experiment is $\sim 2 \mu \mathrm{A}$ and data points below $1 \mu \mathrm{A}$ requires another clibration job. So they are omitted
} 
Table 4.1: Beam current at liquid targets

\begin{tabular}{|c|c|c|}
\hline Angle $\left[^{\circ}\right]$ & $\begin{array}{c}\text { Beam current } \\
\text { at }{ }^{1} \mathrm{H} \text { target }[\mu \mathrm{A}]\end{array}$ & $\begin{array}{c}\text { Beam current } \\
\text { at }{ }^{2} \mathrm{D} \text { target }[\mu \mathrm{A}]\end{array}$ \\
\hline 14.5 & 2.6 & 2.6 \\
\hline 17.0 & 3.2 & 3.2 \\
\hline 21.0 & 5.1 & 5.1 \\
\hline 24.0 & 3.1 & 5.2 \\
\hline 25.5 & 5.3 & 5.3 \\
\hline 30.5 & 2.1 & 2.1 \\
\hline 35.0 & 2.0 & 2.1 \\
\hline 40.0 & 5.2 & 5.1 \\
\hline 45.0 & 7.4 & 10.5 \\
\hline 50.5 & 20.8 & 20.7 \\
\hline 56.0 & 10.2 & 25.9 \\
\hline 62.5 & 10.4 & 26.0 \\
\hline 69.0 & 10.4 & 31.2 \\
\hline
\end{tabular}

\subsubsection{Beam current calibration with silver calorimeter}

Six BCM signals always have some noise and it is mentioned as zero offset here. The zero offset is the $\mathrm{BCM}$ readout with no beam current. It ought to be removed to find the accurate beam current equation.

The zero offset is changing smoothly over time. So zero offsets from cosmic runs and beam-stop moments during ordinary runs are extracted and then the zero offset of each run is determined as the mean value from the two neighboring zero offsets(beam-stop moment or cosmic run). The mean value of BCM d1 zero offset during this experiment is around 156. It is approximately $3 \%$ of $\mathrm{BCM} \mathrm{d} 1$ value of $1.8 \mu A$ runs in Table 4.2.

After the zero offset is subtracted, a linear equation is extracted by comparing bare values of $\mathrm{BCM} \mathrm{d} 1$ and OLO2 beam currents from calibration runs. However this method has several \% uncertainty for low current as it is already mentioned. Silver here. 
Table 4.2: Beam current and BCM d1 from calibration runs

\begin{tabular}{|c|c|c|}
\hline & Beam current $(\mu A)$ & BCM d1 \\
\hline \multirow{4}{*}{ OLO2 } & 71.2 & 160527.2 \\
\cline { 2 - 3 } & 40.1 & 90606.2 \\
\cline { 2 - 3 } & 19.8 & 44736.9 \\
\cline { 2 - 3 } & 10.2 & 23463.2 \\
\cline { 2 - 3 } & 4.8 & 11475.8 \\
\cline { 2 - 3 } & 1.8 & 5294.4 \\
\hline \multirow{4}{*}{$\begin{array}{c}\text { Silver } \\
\text { calorimeter }\end{array}$} & 2.6 & 6100.1 \\
\cline { 2 - 3 } & 1.1 & 2533.2 \\
\cline { 2 - 3 } & 0.5 & 1222.7 \\
\cline { 2 - 3 } & 0.4 & 851.9 \\
\hline
\end{tabular}

calorimeter is eligible to obtain low current down to several hundreds $n A$. It can replace OLO2 monitor in low current region. Fig. 4.1 shows plots of calibration analysis. The $x$ axis is the BCM d1 and the $y$ axis is the beam current. Black symbols are data from OLO2 monitor and red symbols are data from silver calorimeter. The blue line is an extrapolation of the linear fit function made from OLO2 data in the region $I \geq 10 \mu A$. The top panel shows the fitting line and calibration data points. In this picture, it is not clear whether the fitting curve agrees well with data at low current because of the large scale of the $x$ axis. The Bottom panel is focusing on the low current region. Black OLO2 symbols below $10 \mu \mathrm{A}$ deviate from the fit function and the deviation becomes bigger with the decreasing beam current. On the other hand, red symbols of silver calorimeter above $1 \mu \mathrm{A}$ agree with the fit function better than black symbols though the extrapolation curve is made from OLO2 data in the high current region. Below $1 \mu A$, red symbols become higher than the curve and another fitting for the local region is required. Based on this, new data set is chosen as combinations of $\operatorname{OLO} 2 \operatorname{currents}(I \geq 10 \mu A)$ and silver calorimeter currents $(1 \mu A<I<10 \mu A)$. From these, a new fit function of linear equation is made.

The data variation from new fit function are shown in Fig. 4.2. The $y$ axis is the 
percent variation of beam current deviation ( $\left.\frac{\text { data-fit function }}{\text { fit function }}\right)$ and the $x$ axis is the beam current. It is found that the fit function agrees with data within $\pm 0.5 \%$ down to $1 \mu A .^{2}$

Obtained fit function is given by Eq. 4.1. The beam current of each run is calculated from this equation.

$$
I[\mu A]=0.000441682 *\left(\frac{\int(\mathrm{BCM} \mathrm{d} 1-\text { zero offset }) \mathrm{d} \tau}{\int \mathrm{d} \tau}-48.2141\right)
$$

where $\tau$ is the beam time.

\subsection{Target Density Correction}

\subsubsection{Overview}

The Hall A target cryogenic system maintains the density of liquid hydrogen $\left(\rho_{0}^{H}\right)$ and liquid deuterium $\left(\rho_{0}^{D}\right)$ with $0.0723 \mathrm{~g} / \mathrm{cm}^{3}$ and $0.167 \mathrm{~g} / \mathrm{cm}^{3}$ within $0.1 \%$ uncertainty. The operation conditions are 19K, 25psi for hydrogen and 22K, 22psi for deuterium. But the local density near beam passage is changed because the beam delivers some power to the neighborhood target molecules and boil the target. So the target density can be expressed as a function of beam current, $\rho=\rho(I)$. We assume that $\rho(I)$ is a linear function of $\rho(0)$ and $I$. The density of liquid target should be $\rho_{H}(I)$ and $\rho_{D}(I)$ instead of $\rho_{0}^{H}$ and $\rho_{0}^{D}$ in the calculation of cross section.

Runs from the right arm spectrometer are used to find the change of target density by the beam current. During this experiment, the right arm spectrometer is fixed at the scattering angle setting of $24^{\circ}$. So all right arm runs have the same kinematic settings except the beam properties. Ideally all right arm runs from a target should have the same cross section and normalized yield. But the decrease of normalized

\footnotetext{
${ }^{2}$ More data points are measured from the silver calorimeter in the region $I<1 \mu A$. But above fit function does not agree well with data and shows a few $\%$ difference. Hence it is ignored here and another fit function should be made for very low current region alone.
} 

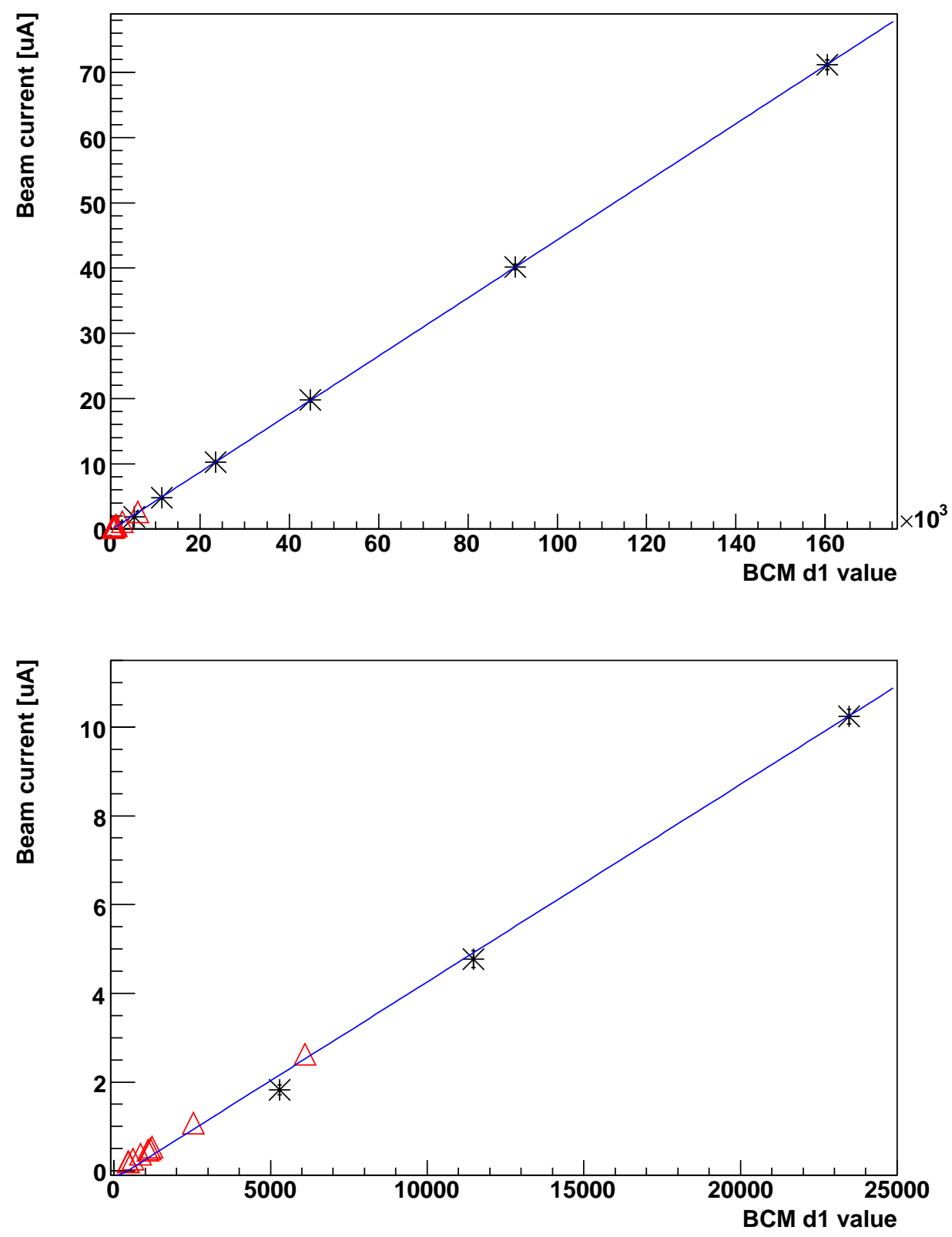

Figure 4.1: Beam current vs. BCM d1 at calibration runs.

Black symbols are data points from OLO2 current and red symbols are silver calorimeter data. The blue line is an extrapolation of linear fit function made from OLO2 data in the region $I \geq 10 \mu \mathrm{A}$. Whole data points are drawn in top panel and bottom panel is focused on low current region. Silver calorimeter data agree with fit function better than OLO2 data. 


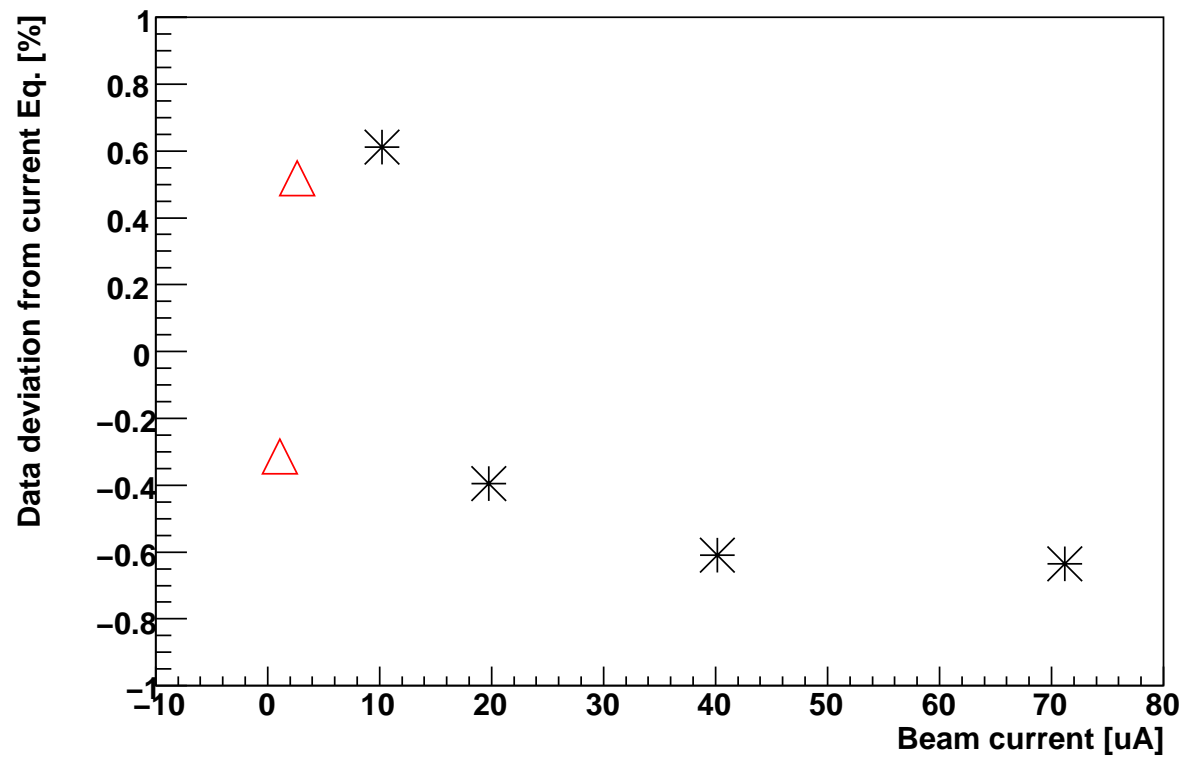

Figure 4.2: Data variation from the new fit function in Eq. 4.1.

The $y$ axis is $\frac{\text { data-fit function }}{\text { fit function }}$ A new data set is chosen from combinations of OLO2 currents $(I \geq 10 \mu A)$ and silver calorimeter currents $(1 \mu A<I<10 \mu A)$. The fit function agrees with data within $\pm 0.5 \%$ down to $1 \mu \mathrm{A}$.

yield was observed with increasing beam current. We can think that the change of target density is the reason for the decrease of normalized yield because all efficiencies such as detector efficiency and beam charge are normalized by the same method. Hence, the linear fitting function can be extracted from the relation of normalized yield variation with beam current and this should be the target density dependence as a function of the beam current.

If a linear fit function $f(I)$ for a liquid target is obtained, the density of liquid target can be written by Eq. 4.2 .

$$
\rho(I)=\rho(0) \times f(I)
$$

The fit function $f(I)$ is extracted by the following method.

1. The $W$ distribution of pure liquid target is obtained after the contribution of 
Al. windows is subtracted. ${ }^{3}$

2. The center of elastic peak $P_{0}$ is found by a fitting process. ${ }^{4}$

3. A peak region in the $W$ distribution is chosen and then the number of events $N_{0}$ in the region is found. ${ }^{5}$

4. The yield $\left(N_{0}\right)$ is normalized by detector efficiencies and beam charge.

5. The change of yield by different beam positions is estimated for each run and then $N_{0}$ is corrected by the estimation. Further explanations are given in the next section.

6. The plot of normalized yield vs. beam current is drawn and a new linear fit function is made.

\subsubsection{Yield change by beam position}

Though all the right arm runs have the same kinematics, beam properties of each run can generate run-to-run variations. Especially the beam position gives the biggest contribution because it changes target variables as explained in section 3.5.

Fig. 4.3 shows the beam position distribution of two consecutive runs from right arm. Beam currents of two runs are similar. But there was an abrupt change of beam position between the two runs. In the top panel, the average beam position of $\mathrm{x}$ is $\sim-1.5 \mathrm{~mm}$ and it is $\sim 1.2 \mathrm{~mm}$ in bottom panel. The difference between them is approximately $2.7 \mathrm{~mm}$.

Fig. 4.4 draws $Q^{2}$ distributions from the two runs. Both distributions are normalized by all factors such as detector efficiency, beam charge and target length. The red distribution is shifted from the black one and the shift is caused by the beam position

\footnotetext{
${ }^{3}$ Further explanations are in section 4.5.2.

${ }^{4}$ Further explanations are in section 4.5.3.

${ }^{5}$ The peak region do not need to be the exact elastic peak for deuteron target, because this job is to find the target density and the yield of inelastic region is also proportional to the target density.
} 

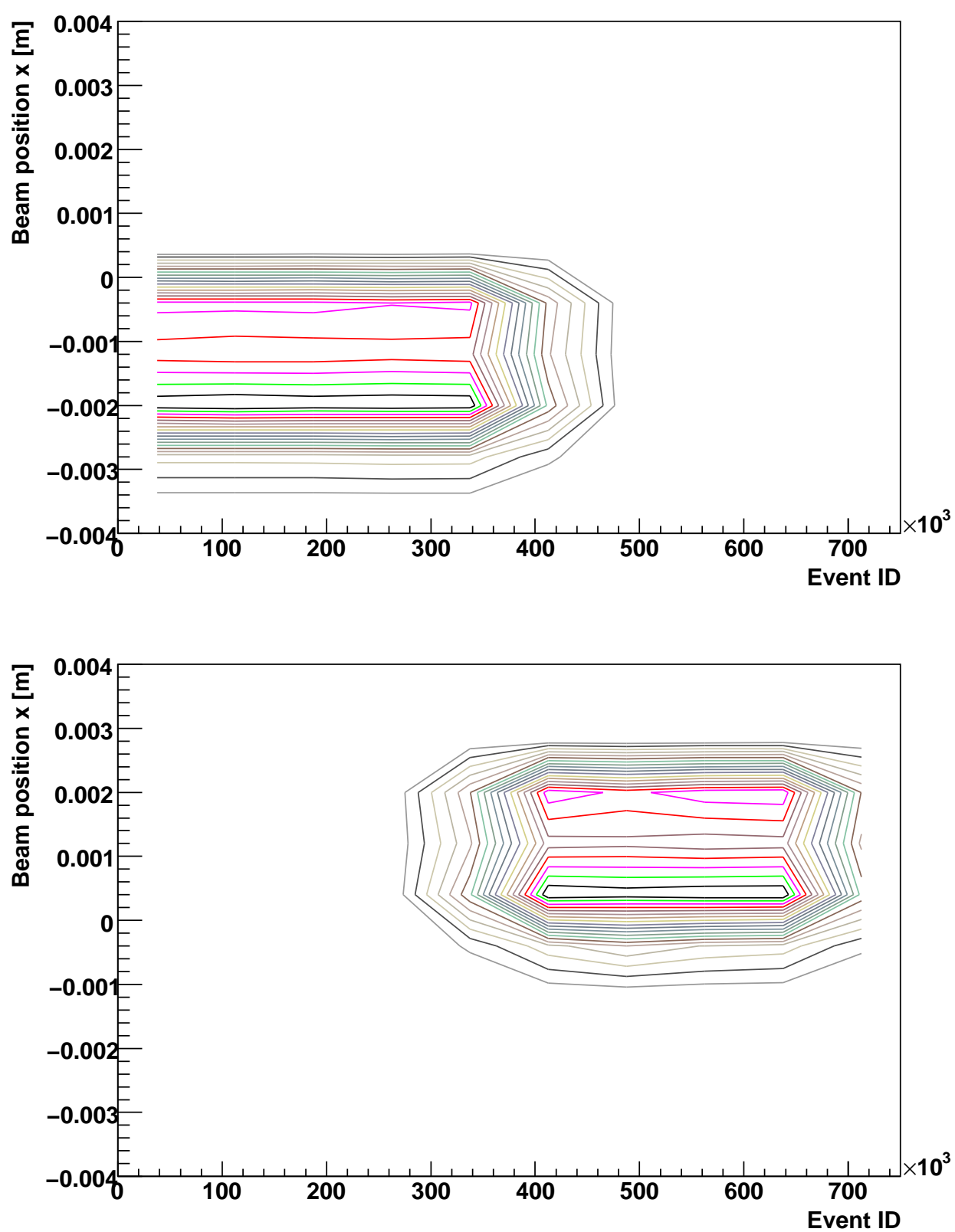

Figure 4.3: Contour plot of beam position. The top panel and bottom panel are from two consecutive runs. The average beam position difference between two runs is approximately $3 \mathrm{~mm}$. 


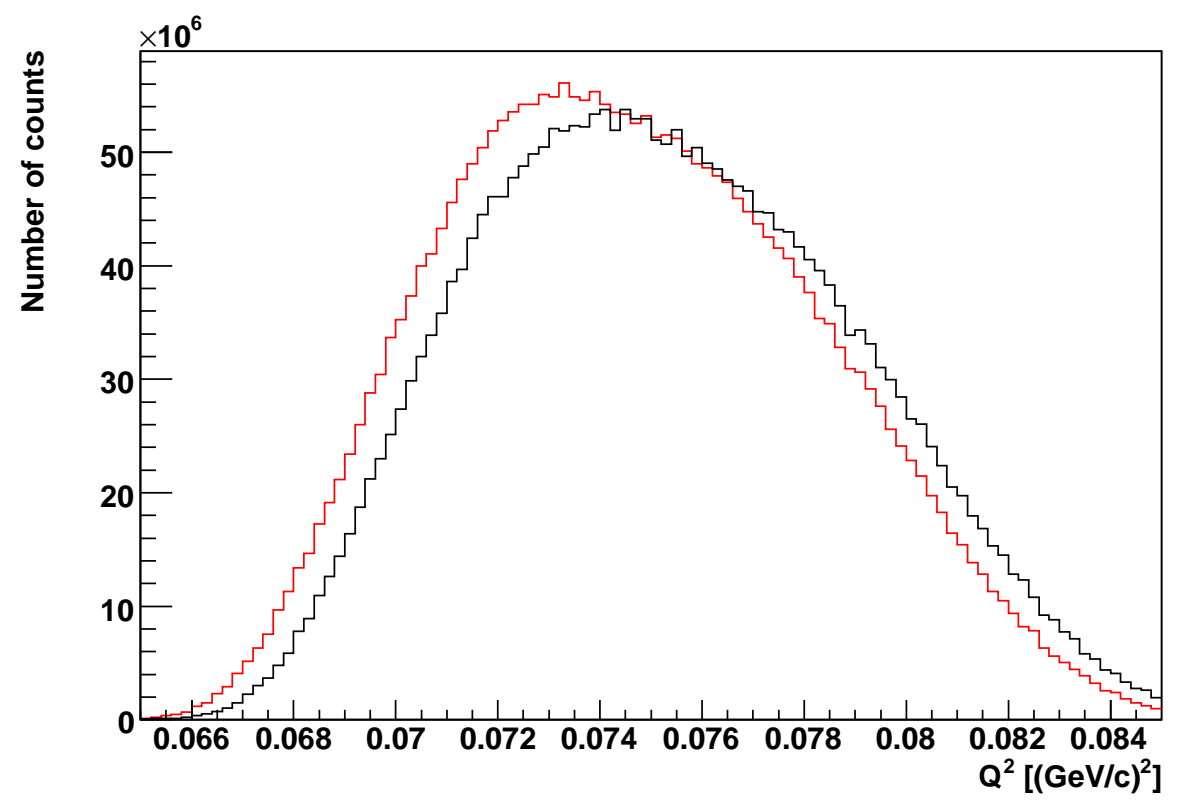

Figure 4.4: The $Q^{2}$ distribution of two consecutive runs in Fig. 4.3. The black curve is shifted from the red curve and they agree in the center region around 0.074 $\left[(\mathrm{GeV} / \mathrm{c})^{2}\right]$. Two distributions have similar cross section values but normalized yields are different because of beam position.

difference. The yield is proportional to the area defined by the $Q^{2}$ distribution curve in the plot. Since the cross section is larger at low $Q^{2}$, the red curve is shifted to the low $Q^{2}$ region and it has larger yield than the black one. On the other hand two curves almost agree in the center region around $0.074(\mathrm{GeV} / \mathrm{c})^{2}$. These features indicate that two curves are generated from similar cross section distributions of $Q^{2}$ but have different normalized yields because of the different $Q^{2}$ value. In the calculation of $\rho(I)$, this effect should be considered.

The yield variation by the beam position change is estimated and it is applied as an efficiency. The method is processed as follows.

1. The average $Q^{2}$ value for a sample run, $\overline{Q_{0}^{2}}$, is calculated.

2. The average $Q^{2}$ value, $\overline{Q^{2}}$, is calculated for every run. 
3. The cross section at $\overline{Q^{2}}$ is obtained for every run. ${ }^{6}$

4. The ratio $\frac{\mathrm{d} \sigma}{\mathrm{d} \Omega}\left(\overline{Q^{2}}\right) / \frac{\mathrm{d} \sigma}{\mathrm{d} \Omega}\left(\overline{Q_{0}^{2}}\right)$ is obtained. Normalized yield of each run, $N_{0}$, is divided by this ratio. This is the correction factor for the beam position variation.

\subsubsection{Target density correction}

Fig. 4.5 shows a plot of normalized yield over beam current for ${ }^{2} \mathrm{D}$ target. The $x$ axis is the beam current in $\mu A$. The $y$ axis is the normalized yield ratio to a sample run. The same color points indicates that their corresponding left arm runs have the same kinematics.

In the top panel, normalized yields are not corrected for the different beam positions. The difference of yield ratio at $3 \mu A$ is $\sim 2.8 \%$. In the bottom panel, the beam position correction is applied and the difference of yield ratio at $3 \mu \mathrm{A}$ has decreased to $\sim 1.1 \%$. It has been observed that the variation of yield at each current becomes smaller after the beam position correction. The density variation by target density change is less than $2 \%$ over $30 \mu A$ for ${ }^{2} \mathrm{D}$ target. A linear fit function $\rho(I)$ is made and it is given by Eq. 4.3.

$$
\rho_{D}(I)=\rho(0) \times(1-0.000493 \cdot I),
$$

where $I$ is the beam current in $\mu A$.

In Fig. 4.6, percent variation of the normalized yield from the fit function is drawn. They agree within $\pm 0.5 \%$. The largest RMS deviation is $0.25 \%$ at the beam current of $5 \mu A$.

The target density change of ${ }^{1} \mathrm{H}$ and ${ }^{12} \mathrm{C}$ are calculated with the same method. Fig. 4.7 shows the result of ${ }^{1} \mathrm{H}$. The top panel is the ratio of the normalized yield vs.

\footnotetext{
${ }^{6}$ Because $Q^{2}$ variation in a kinematics by beam position is very small, cross section of ${ }^{2} \mathrm{D}$ is obtained from world data fit function.
} 

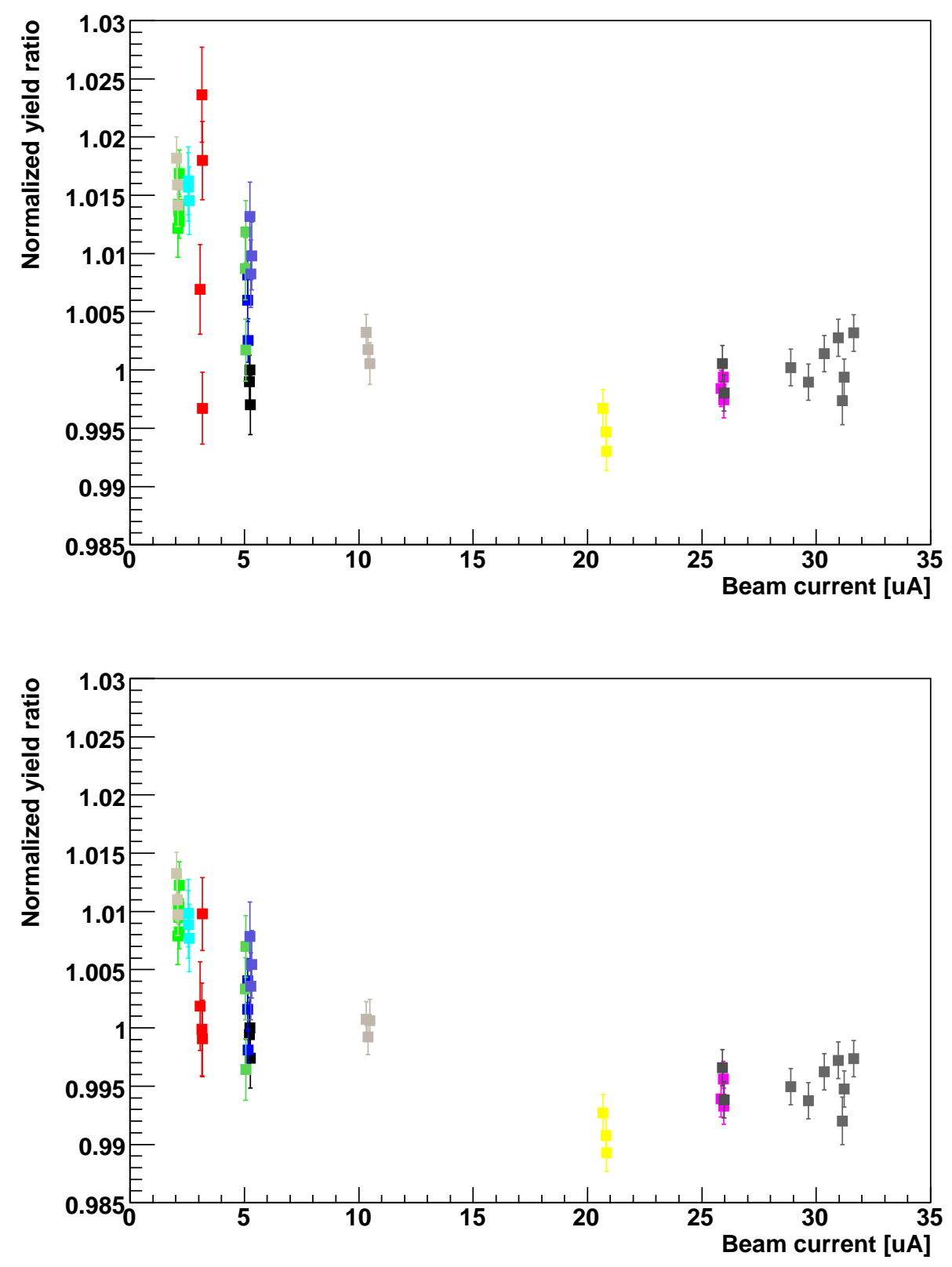

Figure 4.5: Normalized yield vs. beam current for ${ }^{2} \mathrm{D}$ runs.

Yields are expressed as ratio to a sample run. The top panel yield is without the beam position correction and the bottom panel is with the correction. The total density variation is less than $2 \%$ over $30 \mu A$. 


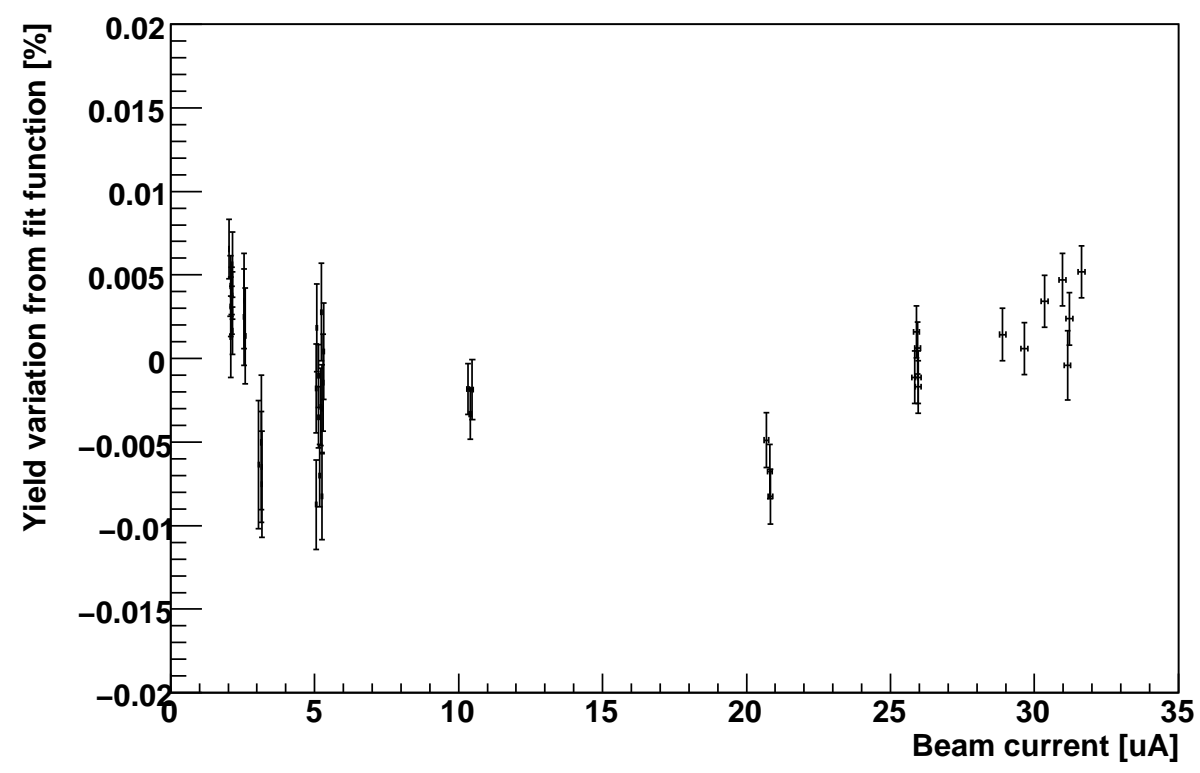

Figure 4.6: The yield variation from the fit function in percentage. The RMS deviation is $0.25 \%$ at $5 \mu \mathrm{A}$.

beam current. The fit function is made from this plot and it is given by Eq. 4.4.

$$
\rho_{H}(I)=\rho(0) \times(1-0.001145 \cdot I)(\operatorname{Iin} \mu A)
$$

The variation over the fit function in percentage is drawn in the bottom panel. The RMS deviation is smaller than that of deuterium.

Fig. 4.8 shows the result for ${ }^{12} \mathrm{C}$. The normalized yield is not proportional to the beam current here. It has the largest deviation at the lowest beam current. It is because the beam current uncertainty is increasing in the low beam current region. 

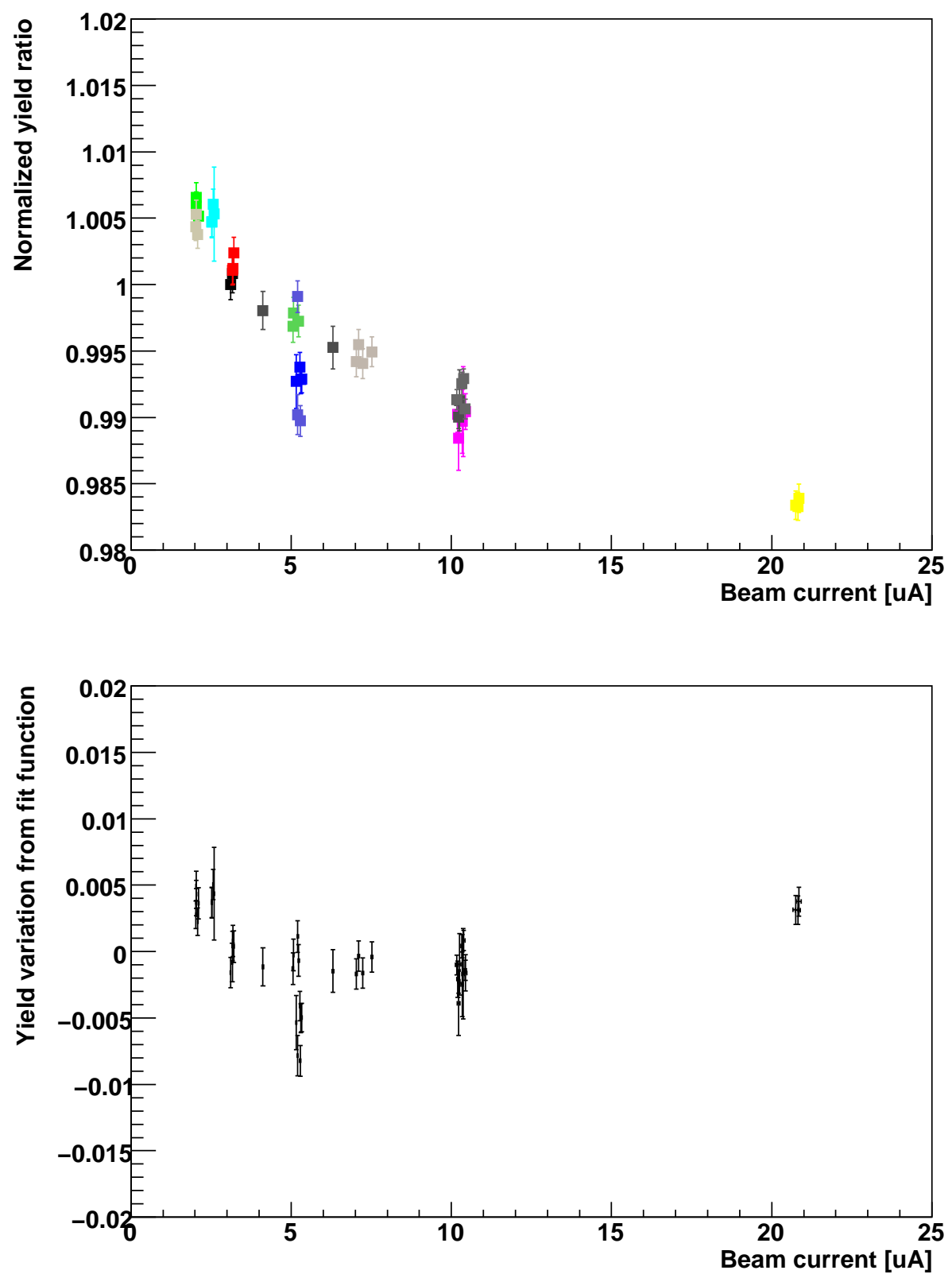

Figure 4.7: Top panel : Normalized yield vs. beam current for ${ }^{1} \mathrm{H}$ runs. Yield are expressed as ratio to a sample run. Bottom panel : The yield variation from the fit function in percentage. 

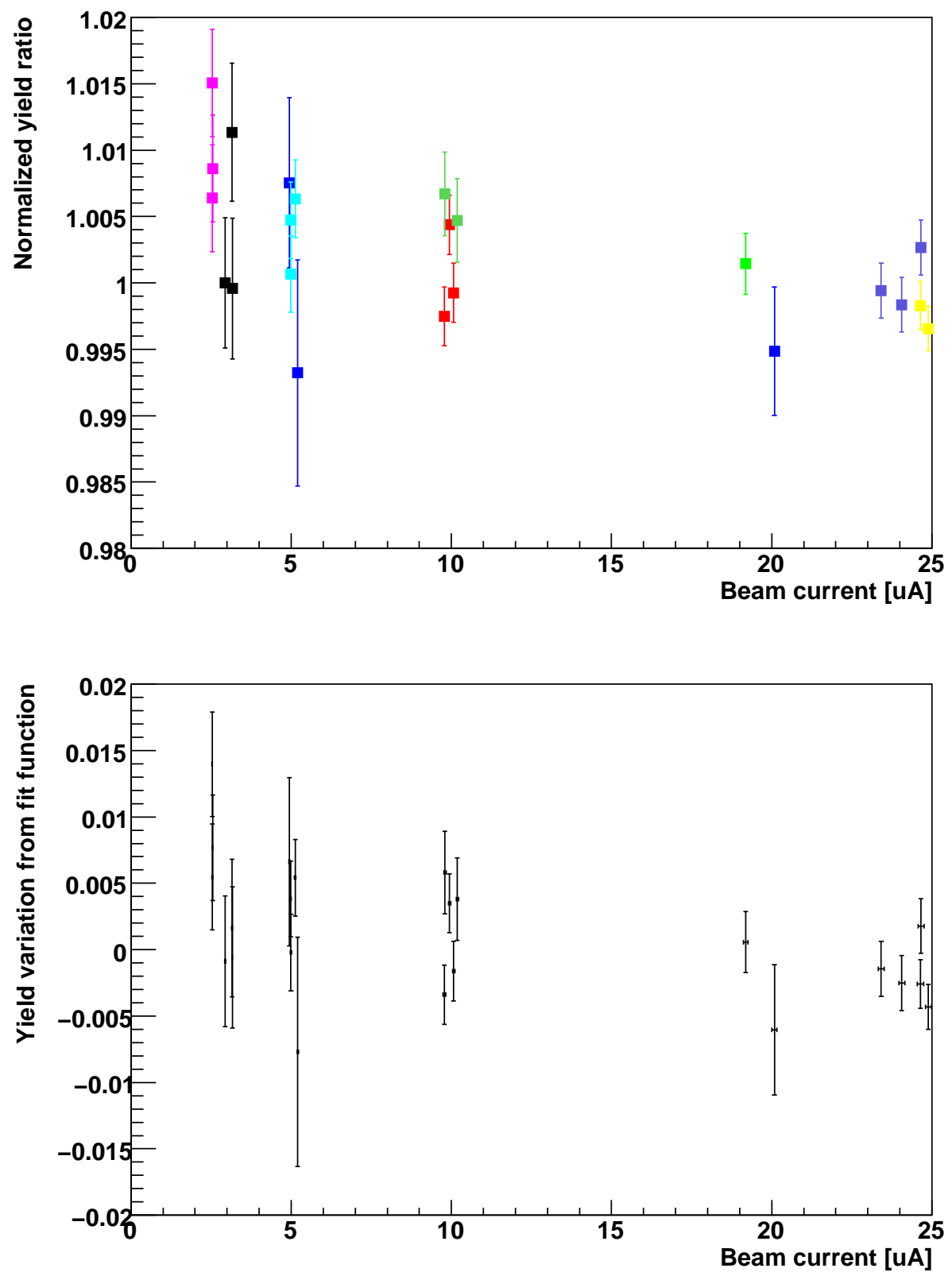

Figure 4.8: Top panel : Normalized yield vs. beam current for ${ }^{12} \mathrm{C}$ runs. Yield is expressed as ratio to a sample run. Bottom panel : The yield variation from the fit function in percentage. The yield is not correlated with beam current. 


\subsection{Target Length Correction}

The two liquid target cells have $2 \mathrm{~cm}$ curvature radius at the back side. If the beam position deviates from the center, the effective target length changes. Both of $\mathrm{x}$ and y positions contribute. The beam position distribution of each run becomes uniform after the BPM cut is applied. Hence target length of each run can be easily corrected from the beam position information as

$$
\bar{l}=\left(l_{0}-r\right)+\frac{\int_{x \min }^{x \max } \sqrt{r^{2}-x^{2}} \mathrm{~d} x}{\int_{x \min }^{x \max } \mathrm{d} x},
$$

where $l_{0}$ is the original target length, $r$ is the curvature radius and $x$ is the beam

position deviation, $x=\sqrt{\operatorname{beam}_{x}^{2}+\text { beam }_{y}^{2}}$. xmax and xmin are the raster size and they are determined from positions at the half maximum of BPM distribution edge peaks.

The calculated correction effects are from $0.1 \%$ to $0.3 \%$.

\subsection{Multi-track Events in High Trigger Rate Runs}

\subsubsection{Overview}

VDCs have all the trajectory information of a scattered electron. Target variables describing scattered electrons are obtained by applying transport matrix to detector variables which can be directly calculated from the VDC tracks. There is an implemented algorithm to find tracks in Analyzer.

Some events have multiple VDC tracks. In such cases, some of them are generated from other noise particles or errors of tracking algorithm but some of tracks are generated from real multiple scattered electrons. The tracking efficiencies obtained in section 3.3.2 are introduced to correct errors of tracking algorithm. But this method uses a constant efficiency and treats as all the multi-track events are counted once by the distribution of good track event. In normal runs of Hall A, the contribution 
Table 4.3: The trigger rate and multi-track ratio at high rate runs

\begin{tabular}{|c|c|c|c|}
\hline Target & Angle $\left[{ }^{\circ}\right]$ & Rate $[\mathrm{kHz}]$ & Ratio $[\%]$ \\
\hline \multirow{4}{*}{${ }^{1} \mathrm{H}$} & 14.5 & 182 & 12.3 \\
\cline { 2 - 4 } & 17 & 103 & 7.5 \\
\cline { 2 - 4 } & 21 & 59 & 4.7 \\
\cline { 2 - 4 } & 24 & 18 & 2.2 \\
\cline { 2 - 4 } & 25.5 & 17 & 5.1 \\
\hline \multirow{4}{*}{$\mathrm{D}$} & 14.5 & 201 & 15.1 \\
\cline { 2 - 4 } & 17 & 111 & 9.1 \\
\cline { 2 - 4 } & 21 & 60 & 5.8 \\
\cline { 2 - 4 } & 24 & 26 & 3.9 \\
\cline { 2 - 4 } & 25.5 & 16 & 3.6 \\
\hline
\end{tabular}

of multi-track events are less than a few $\%$ of the total events and the tracking efficiency can be used. But some kinematics have very high trigger rates and ratio of multi-track events exceed $10 \%$. Table 4.3 shows scattering angle setting, trigger rate and ratio of multi-track at several high rate runs of liquid targets.

In Table 4.3, trigger rates are higher than one hundred $\mathrm{kHz}$ in two kinematics and their multi-track ratios range from $7.5 \%$ to $15.1 \%$. In such high trigger rate, wires do not work correctly and tracking efficiencies become lower than $60 \%$. Fig. 4.9 shows distributions of U1 wire efficiency from two runs. The $x$ axis is the wire number and the $y$ axis is the wire efficiency. The top panel is from a $24 \mathrm{kHz}$ run and efficiencies of most wires are approximately 1 . On the other hand, the bottom panel is from a $200 \mathrm{kHz}$ run and efficiencies of some wire are approximately 0.9 . It is not sure that such wires work normally. The wire efficiency is closely related with the tracking efficiency. So the tracking efficiency should be reconsidered in very high rate runs.

We investigated multi-tack events at high current runs to find cases that an event has two real electron tracks. In such cases, two electrons which are scattered from two individual interaction could come into HRS simultaneously and both of electrons should be counted. So the old tracking efficiency should be corrected for multi-track 

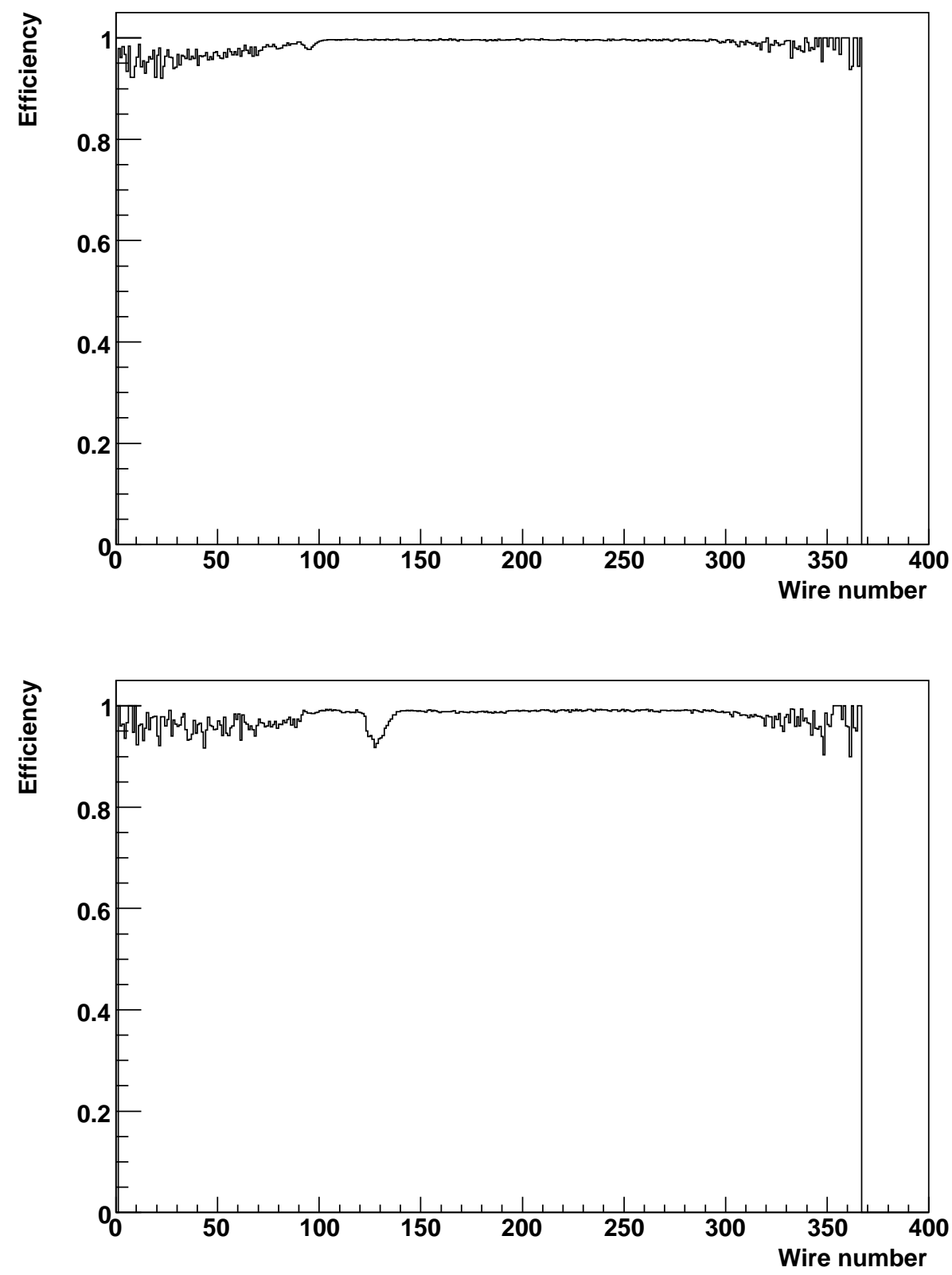

Figure 4.9: U1 wire efficiency.

$x$ axis is wire number and $y$ axis is wire efficiency. Top panel is from a $24 \mathrm{kHz}$ run and bottom panel is from a $200 \mathrm{kHz}$ run. 
events.

The number of real electrons in a multi-track event is judged by deposited energy in a shower detector ${ }^{7}$ and real multi-track events are separated. From this, the new tracking efficiency is calculated. Some multi-track events could be more than two electrons. But the probability is very rare and the cases are not considered.

\subsubsection{Shower detector energy}

The left(right) HRS has two layers of cherenkov shower detectors and they are constructed from 68(128) lead glass blocks. In left arm, both layers give the same information and they are called as preshower and shower here. They detect the energy of a passing particle and can give approximate energy value of each event. So they are usually used for particle identification to remove pions.

If one electron passes the detector, the total deposited energy would be around 650 $\mathrm{MeV}$ at the standard momentum setting of this experiment. If two electrons pass together, the energy would be twice. Because of this property, the deposited energy of a multi-track event is proportional to the real number of electrons. Hence the real electron number of a multi-track event can be deduced by checking the deposited energy in the shower.

During this experiment, the preshower detector worked normally but the shower detector did not work correctly. Hence single preshower energy information is used solely in this analysis and it is anticipated that the total deposited energy would be about one half of the total energy sum of electrons.

The HRS momentum setting is around $650 \mathrm{MeV}$ for high rate runs. The energy distribution of one track events in preshower is expected to be a distribution like Gaussian that has its peak around one half of $650 \mathrm{MeV}$. If there are some events having two electrons, it is expected that the energy distribution would be a dupli-

\footnotetext{
${ }^{7}$ This method is introduced by Jing first. [49] While his method checks the energy of each tracks, we checked the energy sum of all tracks
} 
cation of two Gaussian functions. One is made from one electron events near 650/2 $\mathrm{MeV}$ and another is made from two electron events around $650 \mathrm{MeV}$.

Fig. 4.10 shows two plots of preshower energy distribution and they show the features of multi-track events as they were expected. The top panel is the energy distribution of one track events. There is only one peak which is made from one electron events.

The bottom panel is the energy distribution of multi-track events. There are two peaks in the plot. One peak is centered around $320 \mathrm{MeV}$ and another one is centered around $550 \mathrm{MeV}$. This is lower than the expected position of $650 \mathrm{MeV}$. It is considered that using preshower alone is not enough to detect the total energy of two electrons completely ${ }^{8}$.

A fitting is made with a sum of two Gaussian function curves : green and red. The blue curve is the sum of two Gaussian functions and it agrees well with data distribution. Events in the first peak are one electron events and their second track is made by non-electron particle. On the other hand events in the second peak have two real electrons. This plot indicates that the contribution from two electron events is not negligible. Two electrons, the first(golden) track and the second track, should be counted for the yield calculation.

The preshower energy distribution is checked for every run. Table 4.4 shows the ratio of the number of two electron events to the number of one electron events. In two kinematics, the ratio is larger than $1 \%$ and multi-track events cannot be neglected. Because two Gaussian fit functions are convoluted in the center region, the accurate number of two electron events are determined by the following procedure.

- An energy $E_{1}$ is chosen to make the integral value of the first Gaussian function above $E_{1}$ is less than $1 \%$ of total integral value.

\footnotetext{
${ }^{8}$ We checked the total energy sum distribution of preshower and shower with data of other experiment, CSR in JLab Hall A. [50]. The second peak is placed at the $90 \%$ increased energy from the first peak energy. It is still smaller than the expectation value but the distribution shape is very similar with Fig. 4.10.
} 

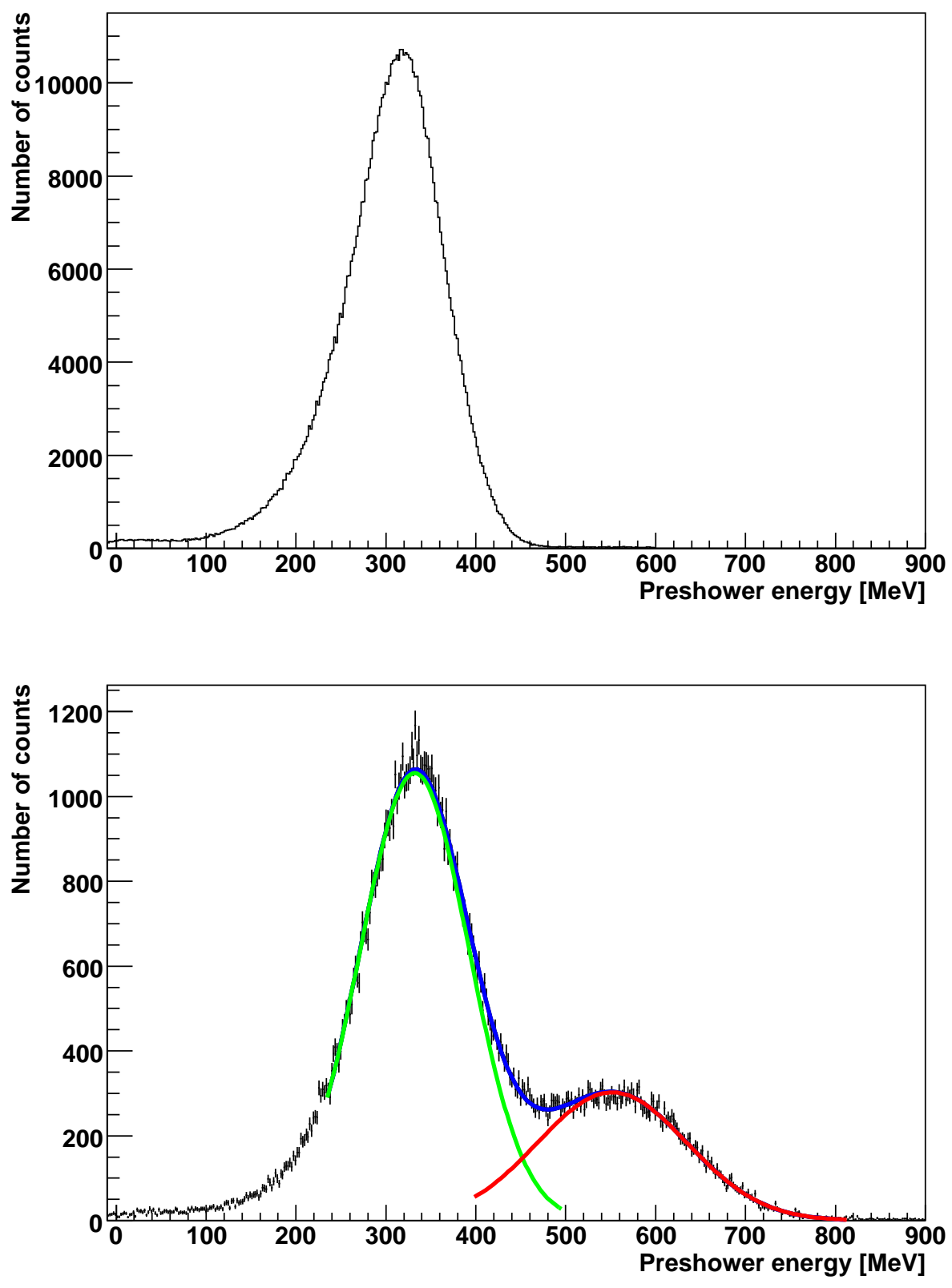

Figure 4.10: Preshower energy distribution.

The top panel is made from one track events. The bottom panel is made from multi-track events. The blue curve is the sum of green curve and red curve. There exist two peaks because of two electrons events. 
Table 4.4: Ratio of two electrons events over one electron event

\begin{tabular}{|c|c|c|}
\hline Target & Rate $(\mathrm{kHz})$ & Ratio of two electrons (\%) \\
\hline \multirow{4}{*}{${ }^{1} \mathrm{H}$} & 182 & 2.9 \\
\cline { 2 - 3 } & 103 & 1.5 \\
\cline { 2 - 3 } & 59 & 0.8 \\
\cline { 2 - 3 } & 18 & 0.2 \\
\cline { 2 - 3 } & 17 & 0.2 \\
\hline \multirow{4}{*}{$\mathrm{D}$} & 201 & 4.0 \\
\cline { 2 - 3 } & 111 & 2.2 \\
\cline { 2 - 3 } & 60 & 1.1 \\
\cline { 2 - 3 } & 26 & 0.5 \\
\cline { 2 - 3 } & 16 & 0.3 \\
\hline
\end{tabular}

- An energy $E_{2}$ is chosen to make the integral value of the second Gaussian function below $E_{2}$ is less than $1 \%$ of total integral value.

- The integral value $A_{1}$ over the first Gaussian function above $E_{1}$ is estimated.

- Events whose energy are lower than $E_{1}$ are considered as one electron events and their numbers are weighted by the ratio of the integral value of data distribution below $E_{1}$ to $A_{1}$.

- The integral value $A_{2}$ of the second Gaussian function below $E_{2}$ is estimated.

- Events whose energy are higher than $E_{2}$ are considered as two electron events and their numbers are weighted by the ratio, the integral value of data distribution above $E_{2}$ to $A_{2}$.

\subsubsection{Tracking efficiency with multi-track}

It is seen from Table 4.4 that the contribution of two electrons are not negligible. Hence two electron events are added as a small correction to one electron events by the following procedure. 
1. One track events are selected and the tracking efficiency of one $\operatorname{track}\left(\varepsilon_{1}\right)$, which is defined in Eq. 3.7, is applied.

2. Two track events are selected by the shower detector condition and then two weight factors $\left(\omega_{1}, \omega_{2}\right)$ are obtained from the preshower energy distribution.

3. The tracking efficiency of two electrons $\left(\varepsilon_{2}\right)$ events is obtained.

$\varepsilon_{2}$ is introduced to correct two electron events. It can be calculated with the same method to obtain the tracking efficiency using the good track. Track cut conditions of two electrons are defined as follows.

- Event type 3

- An event should have two electrons.

- Each wire plane has to contain two clusters.

- Each cluster has the number of hit wires to be from 3 to 6 .

4. $\varepsilon_{1}$ is applied to the first track of two electron events with the weight factor $\omega_{1}$. $\varepsilon_{2}$ is applied to second tracks of two electron events with the weight factor $\omega_{2}$. A new tracking efficiency can be made from combinations of $\varepsilon_{1}, \varepsilon_{2}, \omega_{1}$ and $\omega_{2}$. It can be considered as a constant vector.

5. The distribution of two electron events are corrected with new tracking efficiency. Then, they are added to the one track distribution.

The top panel of Fig. 4.11 is a $W$ distribution of ${ }^{2} \mathrm{D}$ run with the highest trigger rate. All detector efficiencies are included and the elastic region is chosen. The black line is before counting multi-track events and the red line is after counting multitrack events. The yield has increased after multi-track events are included. The bottom panel is the $Q^{2}$ distribution of elastic region. Selected elastic region is the $W$ region in top panel. The ratio of the two panels is drawn in Fig. 4.12. Average increase of the yield is approximately $7 \%$ in the center region which corresponds 
to the meaningful acceptance region. This is larger than the ratio of two electron events $(4 \%)$. It is considered that events in elastic peak have higher rate than inelastic events and they occupy most of the two electron events.

\subsection{Elastic Peak}

\subsection{1 $W$ spectrum}

The invariant mass $W$ is a good variable to describe the characteristics of target nucleus. From a $W$ distribution, available excited states of the target nucleus can be shown and the type of the interaction, elastic or inelastic or excited states, can be inferred.

Five targets, ${ }^{1} \mathrm{H},{ }^{2} \mathrm{D},{ }^{12} \mathrm{C},{ }^{27} \mathrm{Al}$ and ${ }^{181} \mathrm{Ta}$, are used in this experiment. Fig. 4.13 Fig. 4.17 show $W$ distributions of all targets and they are made at the lowest angle. Peculiarities of each target nucleus are shown in the plots.

${ }^{1} \mathrm{H}$ consists of one nucleon and no excited state exists. Instead the distribution of ${ }^{27} \mathrm{Al}$ are convoluted because ${ }^{1} H$ is in liquid state and it is contained in an Aluminum cell.

A $W$ distribution of ${ }^{1} \mathrm{H}$ is drawn in Fig. 4.13. The elastic peak is positioned at the mass of ${ }^{1} \mathrm{H}$ and the radiation tail of the peak is seen. Below the mass of ${ }^{1} \mathrm{H}$ where scattering events from ${ }^{1} \mathrm{H}$ are prohibited, the contribution from ${ }^{27} \mathrm{Al}$ are shown and the radiation tail of it are convoluted into the ${ }^{1} H$ distribution. The method to remove ${ }^{27} \mathrm{Al}$ contribution is discussed in the following sections 4.5.2 and 4.5.5.

The main target ${ }^{2} \mathrm{D}$ is the only nucleon-nucleon system and the binding energy between the two nucleons is $2.22 \mathrm{MeV}$. Because of its small binding energy, no excited state of ${ }^{2} \mathrm{D}$ exists. If an electron delivers energy larger than the binding energy to the ${ }^{2} D$ nucleus, it causes the disintegration of the nuclear system. The binding energy is the threshold of inelastic scattering from the elastic peak. Because this threshold is only $2.22 \mathrm{MeV}$ apart from the elastic peak, inelastic events are mixed 

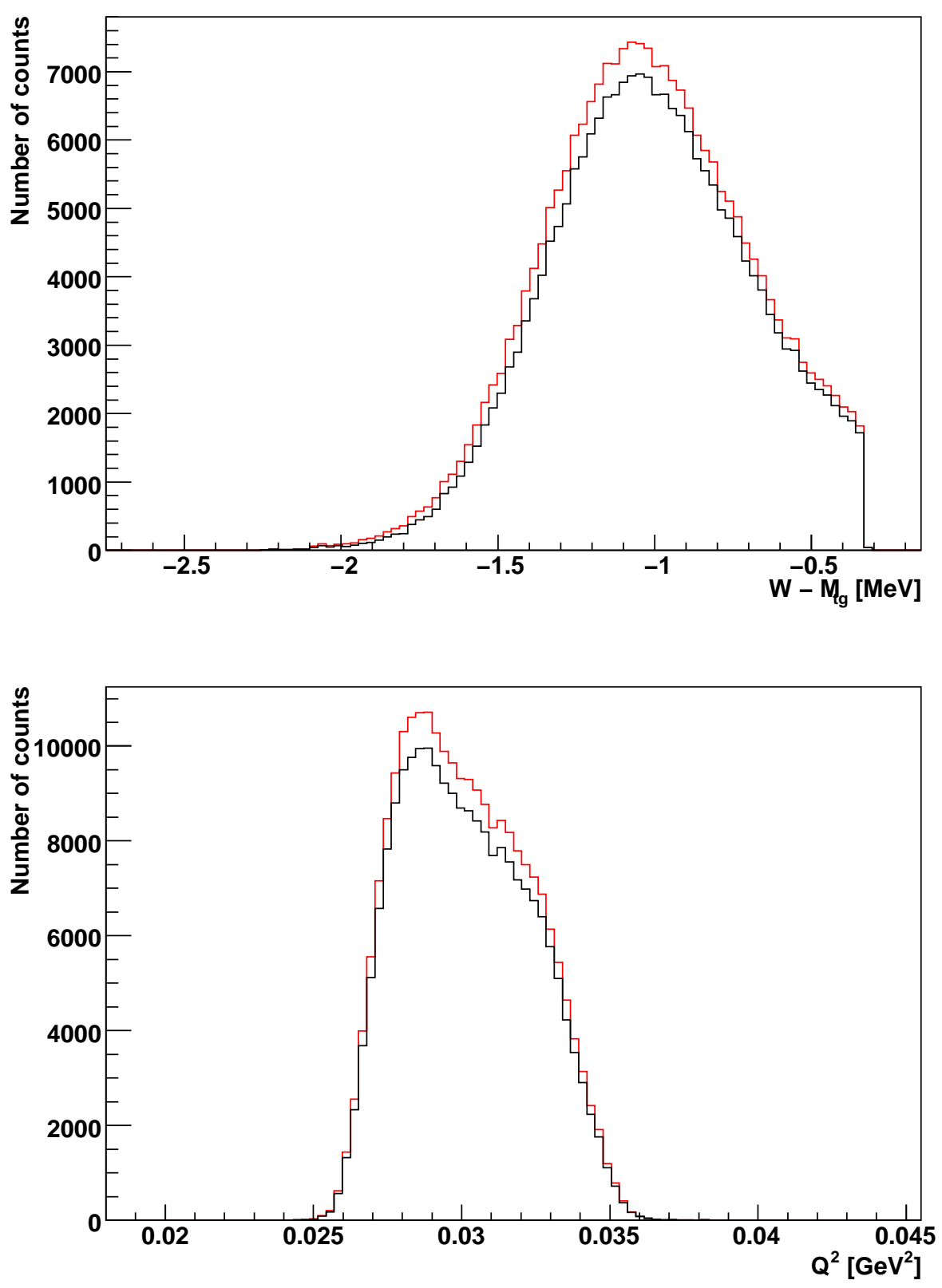

Figure 4.11: Top : $W$ distribution of $\mathrm{a}^{2} \mathrm{D}$ run at the highest trigger rate. Bottom : $Q^{2}$ distribution of a ${ }^{2} \mathrm{D}$ run at the highest trigger rate. The black line is before the multi-track correction and red line is after the correction. 


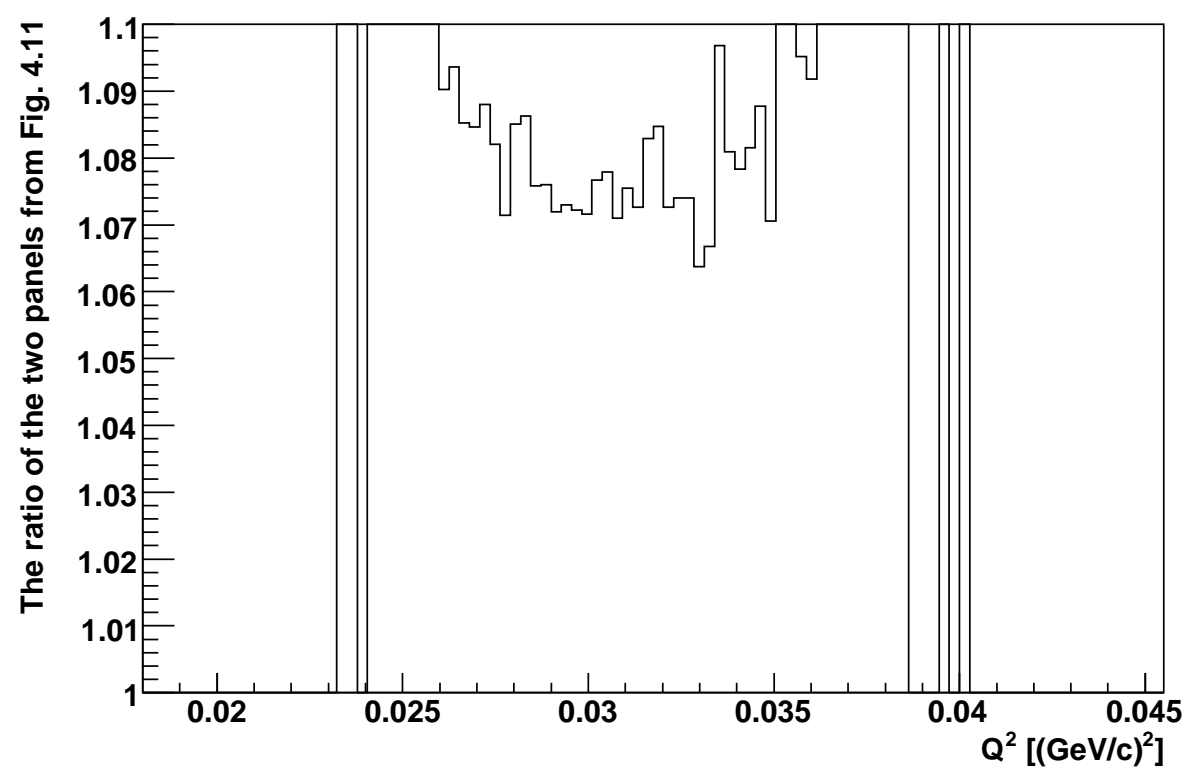

Figure 4.12: The increment ratio of $Q^{2}$ distribution after multi-track correction is applied. Ratios are approximately $7 \%$ in the center region(meaningful acceptance region).

with events of elastic peak and the radiation tail. Selecting pure elastic events is the main job of the yield extraction at deuterium target. Further explanations are in section 4.5.4. Fig. 4.14 is a $W$ distribution of ${ }^{2} D$. Elastic peak of ${ }^{2} D$ comes first and it is followed by inelastic events after the threshold. ${ }^{2} D$ target is also in liquid state and the aluminum peak is shown below the mass of ${ }^{2} D$.

While light atoms like ${ }^{1} \mathrm{H}$ and ${ }^{2} \mathrm{D}$ have no excited state, ${ }^{12} \mathrm{C}$ has many excited states. The excited state energies of ${ }^{12} \mathrm{C}$ are already well known [51]. Especially three excited states exist within $10 \mathrm{MeV}$ from the ground state. The values of excitation energy are $4.44 \mathrm{MeV}, 7.65 \mathrm{MeV}$ and $9.64 \mathrm{MeV}$. Fig. 4.15 is a $W$ distribution of ${ }^{12} \mathrm{C}$ and energy differences agree well with known values. Red symbols are marked at the ground state and three excited states.

Fig. 4.16 is a $W$ distribution of ${ }^{27} \mathrm{Al}$. Because the end cap cells of liquid target 


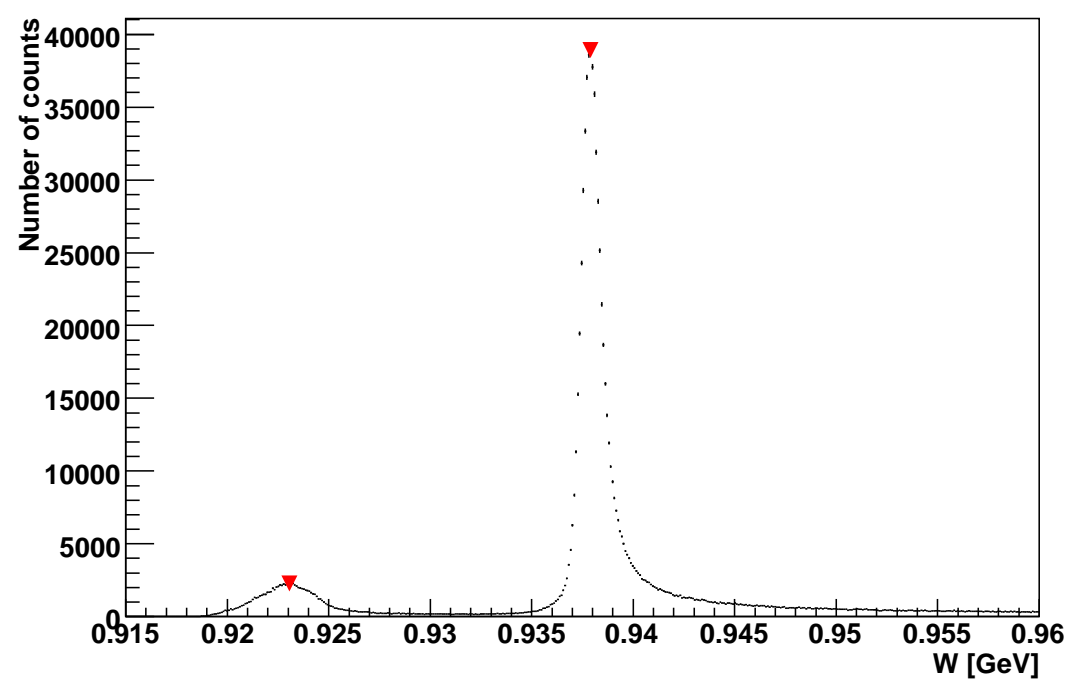

Figure 4.13: $W$ distribution of ${ }^{1} \mathrm{H}$.

The elastic peak and radiation tail of ${ }^{1} \mathrm{H}$ are shown. Below elastic peak, there are events from Al. windows and they are convoluted with ${ }^{1} \mathrm{H}$ data.

are aluminum, ${ }^{27} \mathrm{Al}$ distribution is made to subtract contributions from the end cap cells which are entangled with elastic events of ${ }^{1} H$ and ${ }^{2} D$.

Fig. 4.17 is a $W$ distribution of ${ }^{181} \mathrm{Ta}$ and it shows many excited states.

\subsubsection{End cap subtraction in $W$ spectrum}

Targets of ${ }^{1} \mathrm{H}$ and ${ }^{2} \mathrm{D}$ are in liquid state and they are contained in aluminum cells. In the scattering interaction of liquid target, the aluminum cell also interacts with electron beam. From here, the target is mentioned as LH2 or LD2 if the aluminum cell contribution of liquid target is included to a distribution. On the other hand the target is mentioned as ${ }^{1} \mathrm{H}$ or ${ }^{2} \mathrm{D}$ if the contribution of pure hydrogen or deuteron is shown alone. In the scattering of LH2 and LD2, background events scattered from aluminum walls can be removed directly by applying a cut over the range of reaction position z. But if the cut is applied, many events from ${ }^{1} \mathrm{H}$ and ${ }^{2} \mathrm{D}$ are lost and it is hard to preserve a good statistics because the resolution of reaction position $\mathrm{z}$ is 


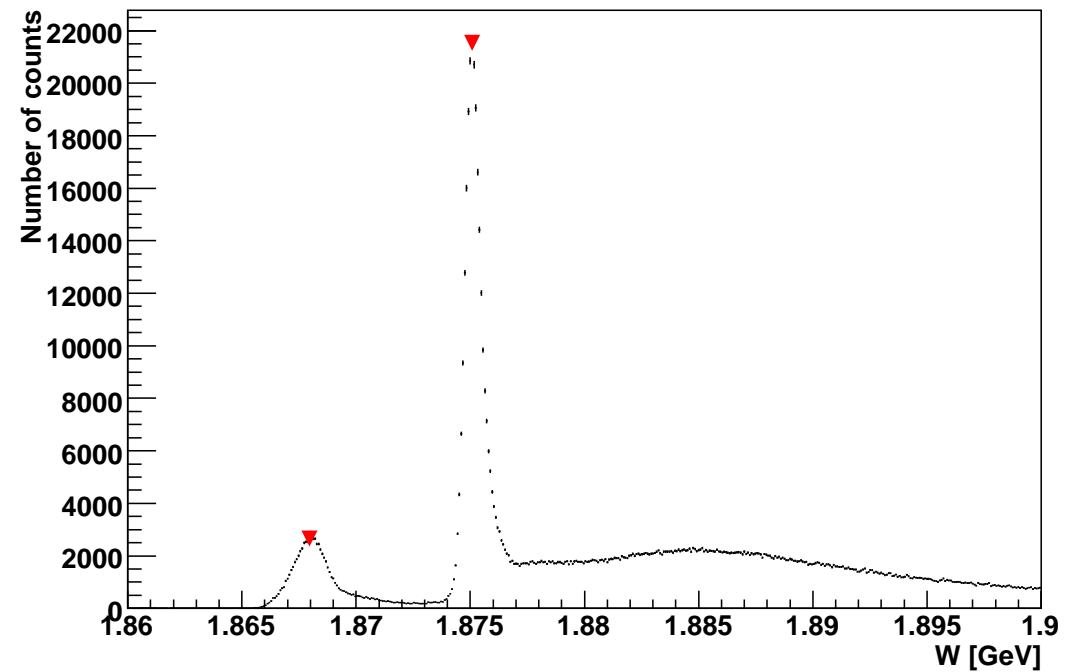

Figure 4.14: $W$ distribution of ${ }^{2} \mathrm{D}$.

There is an elastic peak and it starts to disintegrate $2.22 \mathrm{MeV}$ apart from the peak. Inelastic events are shown above the peak. Below elastic peak, there are events from $\mathrm{Al}$. windows and they are convoluted with ${ }^{2} \mathrm{D}$ data.

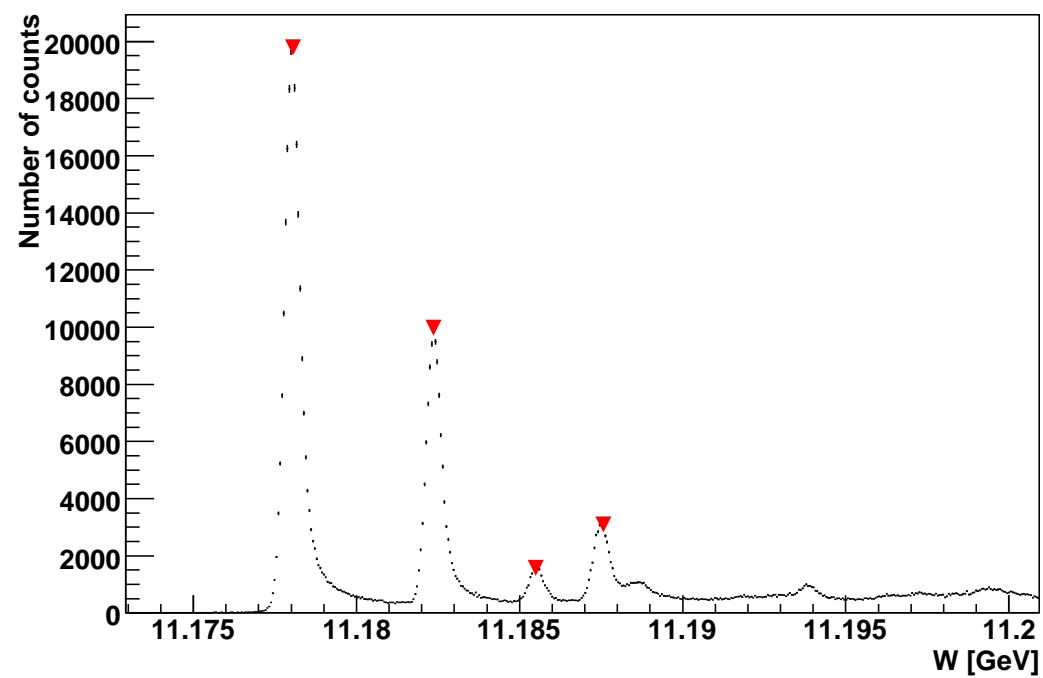

Figure 4.15: $W$ distribution of ${ }^{12} \mathrm{C}$.

${ }^{12} \mathrm{C}$ has many excited states. Especially there are three states within $10 \mathrm{MeV}$ apart from the ground state at $4.44 \mathrm{MeV}, 7.65 \mathrm{MeV}$ and $9.64 \mathrm{MeV}$. 


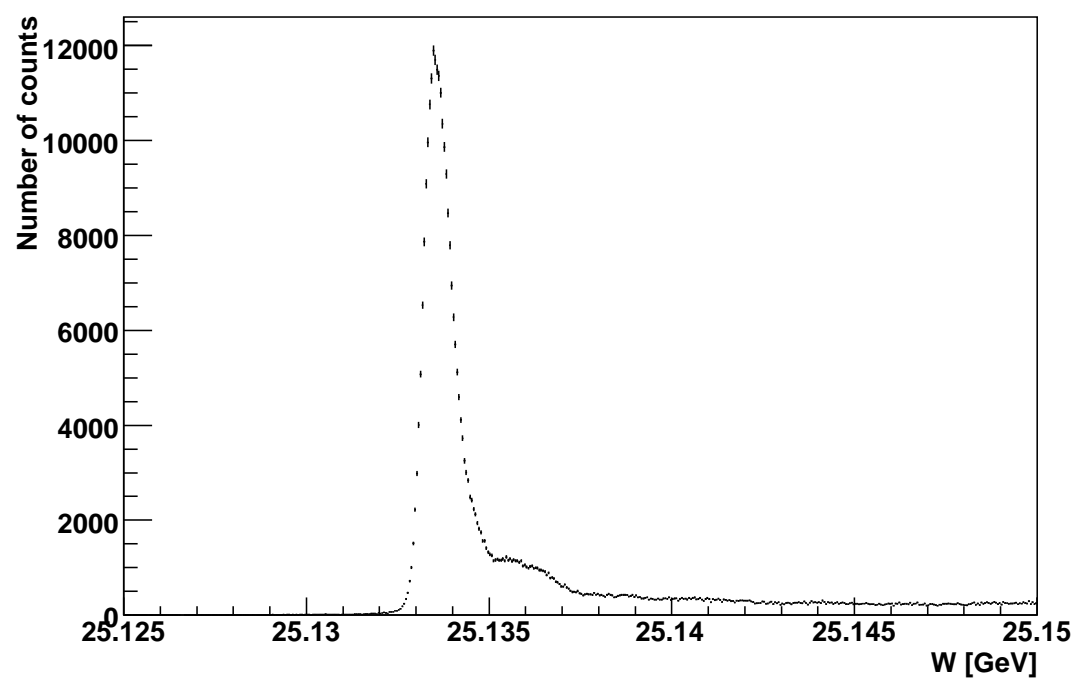

Figure 4.16: $W$ distribution of ${ }^{27} \mathrm{Al}$.

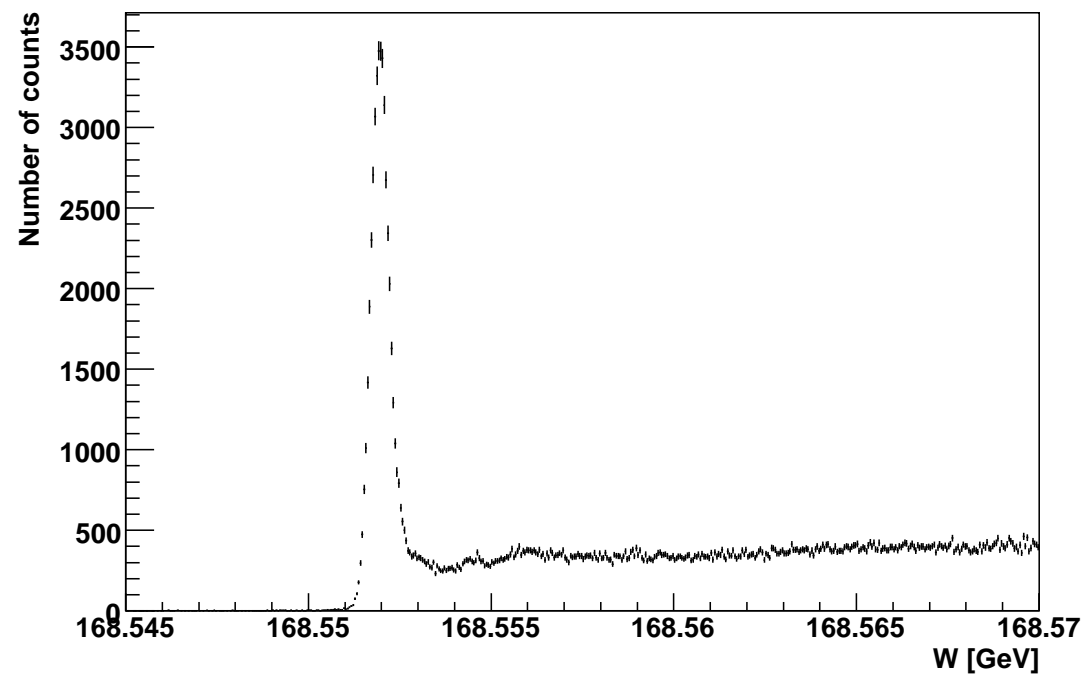

Figure 4.17: $W$ distribution of ${ }^{181} \mathrm{Ta}$. 
approximately $1 \mathrm{~cm}$ and the target length of LH2 and LD2 are $4 \mathrm{~cm}$.

Another method has been used instead. Scattering with aluminum dummy target is measured separately for all kinematics during this experiment. The dummy target is made of $4 \mathrm{~cm}$ length aluminum cell which is the same length of target LH2 and LD2. The window thickness is $0.96 \mathrm{~mm}$ to increase the event rate from the dummy target ${ }^{9}$. And then the aluminum contributions in data of LH2 and LD2 are subtracted by data of dummy run. From here, container walls of liquid target are called as 'Al. windows' and container walls of dummy target are called as 'dummy walls' to discern them.

In this section and section 4.5.5, subtraction methods are discussed and two weight factors $\omega(W)$ and $\omega\left(Q^{2}\right)$ are introduced. They are applied to each event as a variable efficiency depending on $W$ or $Q^{2} . \omega(W)$ is explained here and $\omega\left(Q^{2}\right)$ is presented in section 4.5.5.

The Subtraction in $W$ spectrum is processed by the following procedure and it is described in Fig. 4.18 - Fig. 4.20.

1. A value of invariant mass, $W_{\mathrm{Al}}$, is chosen to separate the peak from Al. windows and the peak from ${ }^{1} \mathrm{H}$ or ${ }^{2} \mathrm{D}$. This is the green vertical line in Fig. 4.18.

2. $W$ distributions of liquid target run and dummy run are drawn with the same cut of "W< $W$ Al." like panels in Fig. 4.18 and Fig. 4.19. $W$ should be calculated with the mass of corresponding liquid target in dummy runs.

3. The number of dummy events are normalized to the number of liquid target events :

- A ratio $\varepsilon_{\text {dum }}$ is obtained between two above $W$ spectrum distributions, $\varepsilon_{\text {dum }}=\frac{\text { number of events from dummy run }}{\text { number of events from liquid target runs }}$.

- The number of events is scaled by $\varepsilon_{\text {dum }}$ in dummy run. It is shown in bottom panel of Fig. 4.19.

\footnotetext{
${ }^{9}$ The thickness of LH2 and LD2 is $0.13 \mathrm{~mm}$.
} 

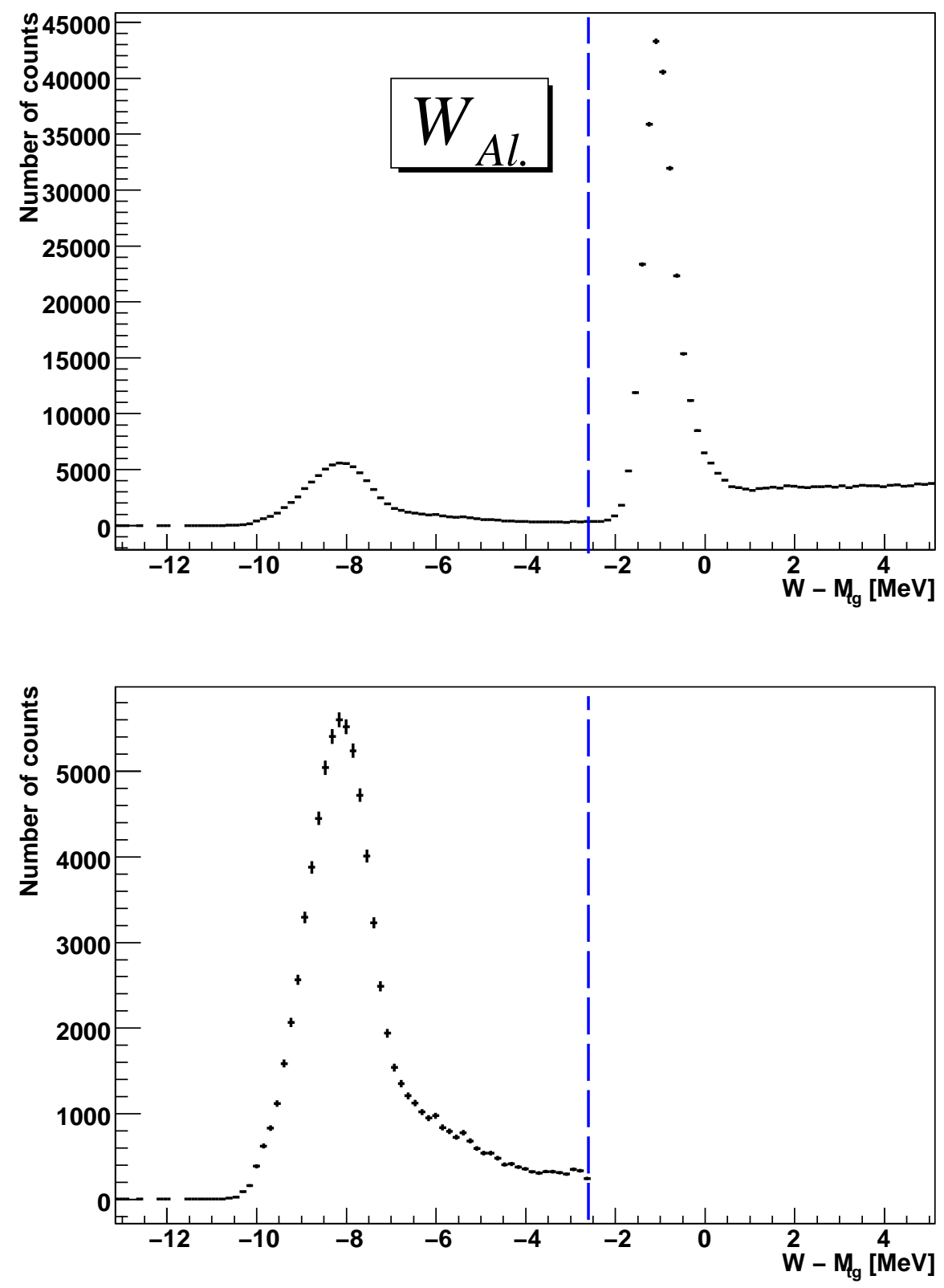

Figure 4.18: Procedure of subtracting Al. windows from liquid target data : The black points are LD2 events before subtraction. The vertical line defines $W_{\mathrm{Al}}$. which separates $\mathrm{Al}$. windows from ${ }^{2} \mathrm{D}$ events. The top panel represents the $W$ distribution of $\mathrm{a}^{2} \mathrm{D}$ run. There are two peaks from $\mathrm{Al}$. walls and ${ }^{2} \mathrm{D}$. The bottom panel shows Al. windows distribution after the cut $W<W_{\text {Al. }}$ is applied. 

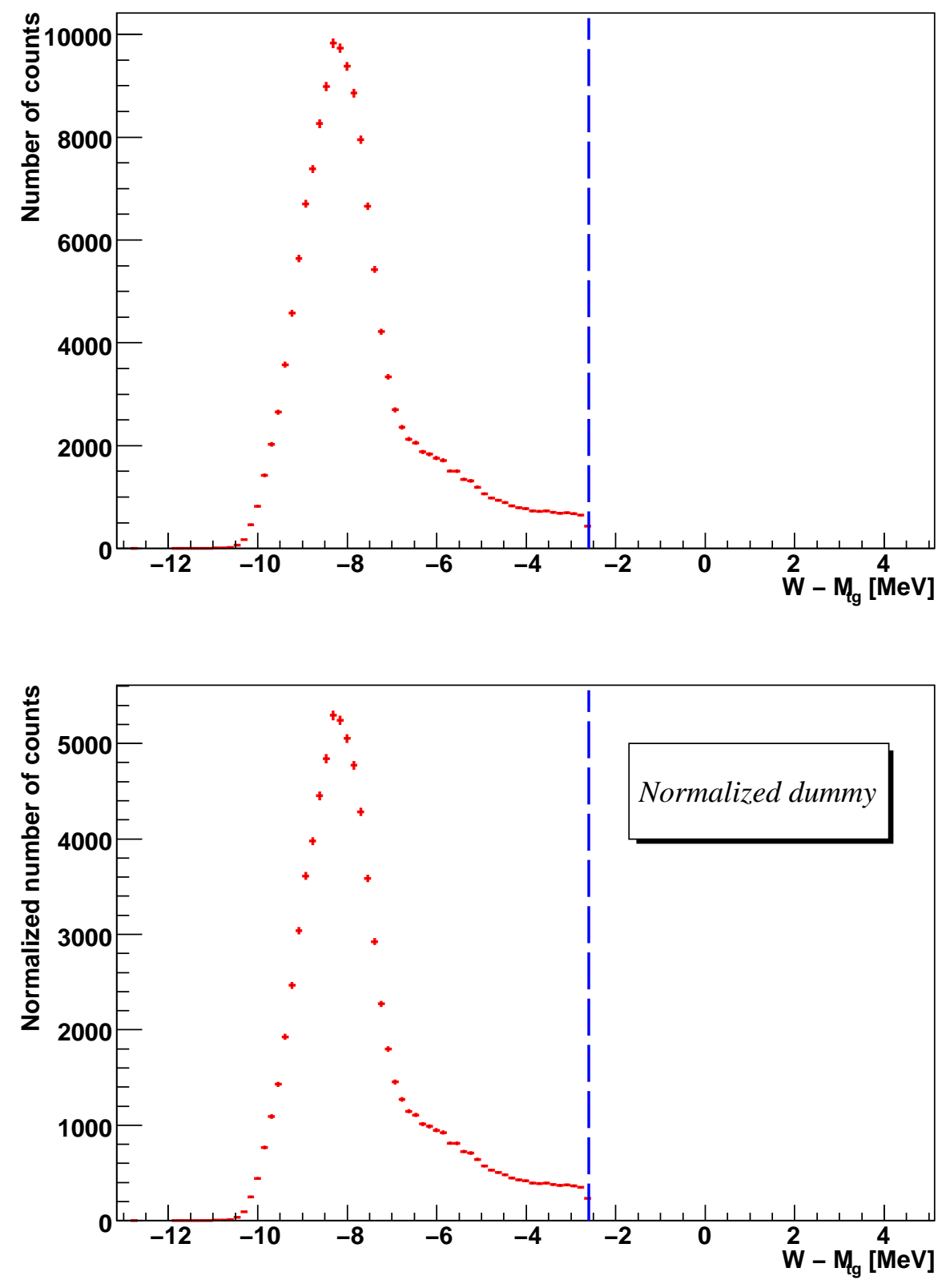

Figure 4.19: Procedure of subtracting Al. windows from liquid target data : The red points are dummy events. The vertical line is the same the value in Fig. 4.18. The top panel shows dummy walls distributions after the cut $W<W_{\mathrm{Al}}$. is applied. The bottom panel is the distribution of normalized dummy walls and it has the same number of events with scaled Al. windows. 


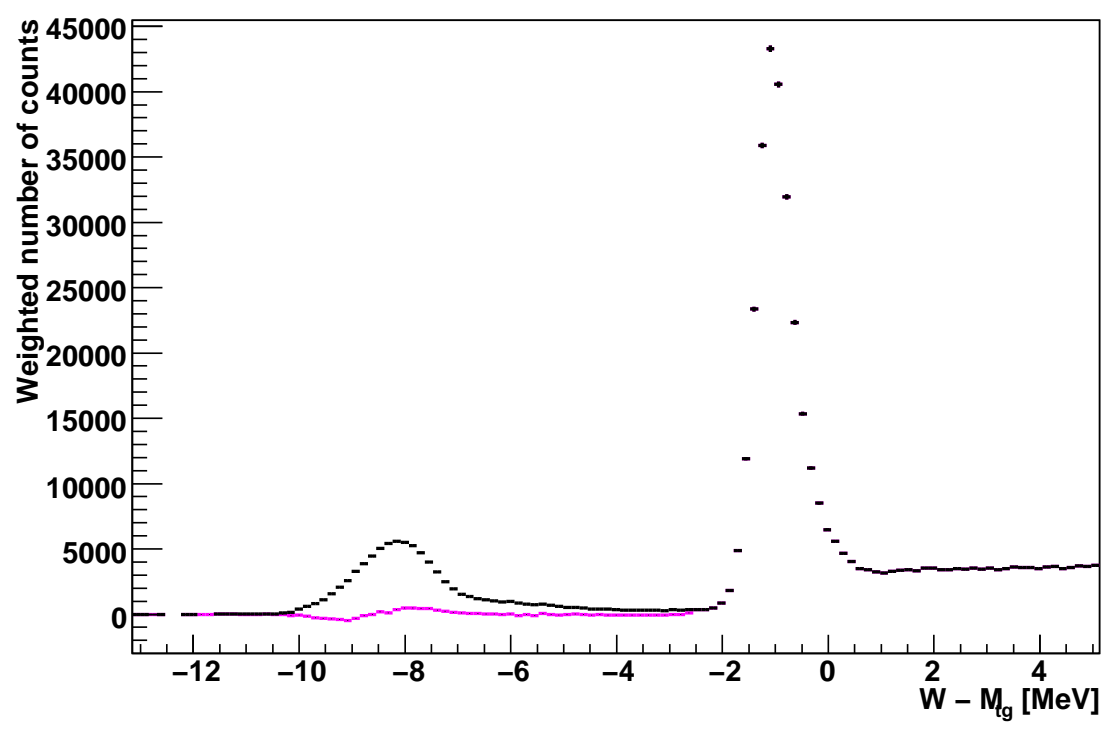

Figure 4.20: The result of subtracting Al. windows from liquid taret data: The black and pink points are duplicated. The black one is from LD2 events before subtraction. The pink one is the ${ }^{2} D$ distribution after subtraction. Events from Al. windows are almost removed.

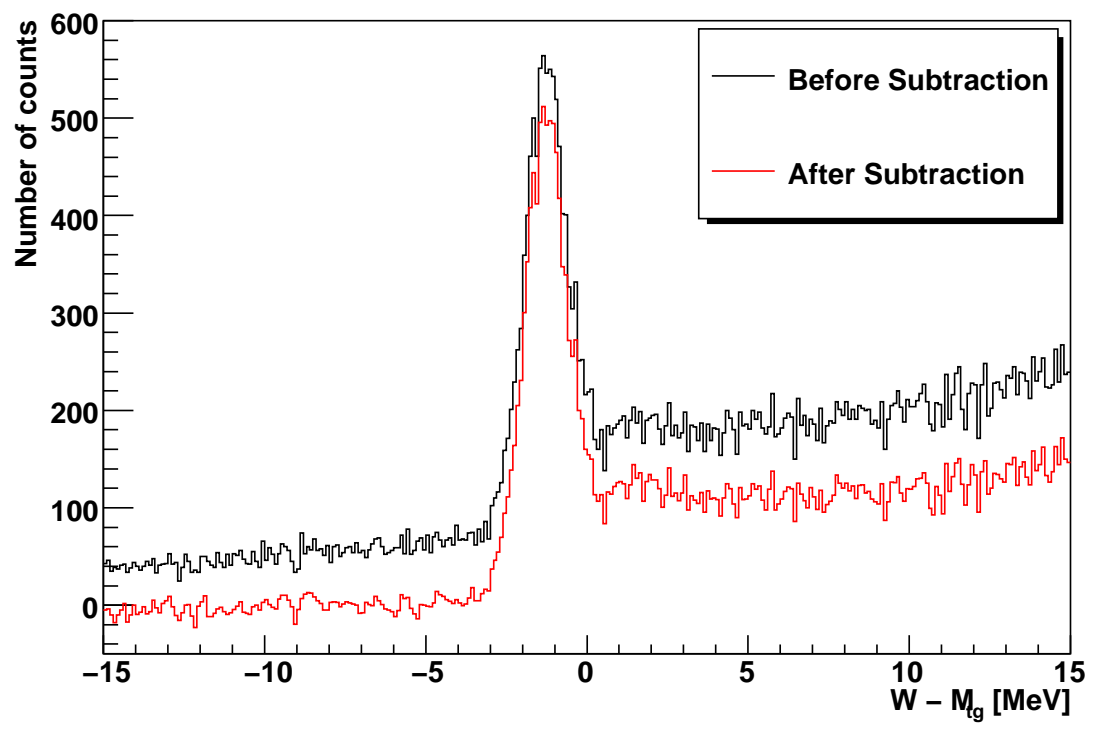

Figure 4.21: The change of $W$ distribution at the highest angle. 
4. The scaled dummy histogram is subtracted from the liquid target histogram and then variable efficiency $\omega(W)$ which is the ratio of change at each $W$ bin is obtained.

$\omega(W)=\frac{\text { liquid target histogram after subtraction }}{\text { liquid target histogram before subtraction }}$

Fig. 4.20 is a distribution of ${ }^{2} D$ after the weight function $\omega(W)$ is applied. The black histogram is a distribution of LD2(before subtraction) and the pink histogram is a distribution of ${ }^{2} D$ distribution(after subtraction). It is seen that events from Al. windows are successfully removed.

In this kinematics, the contributions from Al. windows are small and the two histograms(before and after subtraction) are similar in the region of elastic and inelstic events. There are more background events from Al. walls for ${ }^{2} D$ run at high angle kinematics. Fig. 4.21 shows the change of $W$ distribution from a ${ }^{2} D$ run at the highest angle. After the subtraction, the remaining background contribution convoluted into elastic peak is calculated. It can be calculated with the ratio of (integral value of background)/(integral value of elastic peak). Contributions are kept below $0.15 \%$ for all runs.

Events from dummy walls and Al. windows are different in two aspects. So modified distributions of dummy walls are used in the previous procedure.

1. The existence of liquid target :

- Beam electrons which passed the front Al. window travel through the liquid target.

- Electrons which are scattered at the front wall travel through the liquid target.

If electrons pass through a matter, they are affected by the target nucleus. And three changes are generated : energy loss, straggling and Bremmstrahlung interactions.

2. The different thickness of aluminum : 
The thickness of dummy walls are approximately 7 times larger than one of Al. windows. It gives rise to different effects to the beam and scattered electrons.

Fig. 4.22 draws four $W$ histograms from front dummy wall, back dummy wall, front Al. window and back Al. window. It is made from a run with the lowest angle. Differences among them are prominent. The blue histograms are from Al. windows of liquid target and the red histograms are from dummy walls of dummy target. The front Al. window and dummy wall are drawn by lines and the back Al. window and dummy wall are drawn by triangle symbols. The total number of events from dummy walls is normalized to one from the two Al. windows for comparison. The top panel is from the lowest angle run and the bottom panel is from the highest angle run.

The front dummy wall have much more events than the back dummy wall. This phenomena of dummy target is natural because the front wall has smaller scattering angle than the back wall and it results in smaller $Q^{2}$ and higher event rates. On the other hand the front window and the back window have similar number of events. As scattering angle increases, the $Q^{2}$ difference between front wall and back wall become larger.

Fig. 4.23 is made from the highest angle run. It shows that the number of events from the front wall exceeds the one from the back wall. This corresponds to all targets. The ratio of front window to back window is different for two targets. This difference is generated from the existence of liquid target material and the different thickness of aluminum. In addition, distributions are shifted for liquid target.

To resolve the problem of distribution difference shown above, two modifications of portion controlling and position shifts are applied to dummy target data before the process of subtraction.

By the previous method, the weight function $\omega(W)$ for liquid target is obtained. 


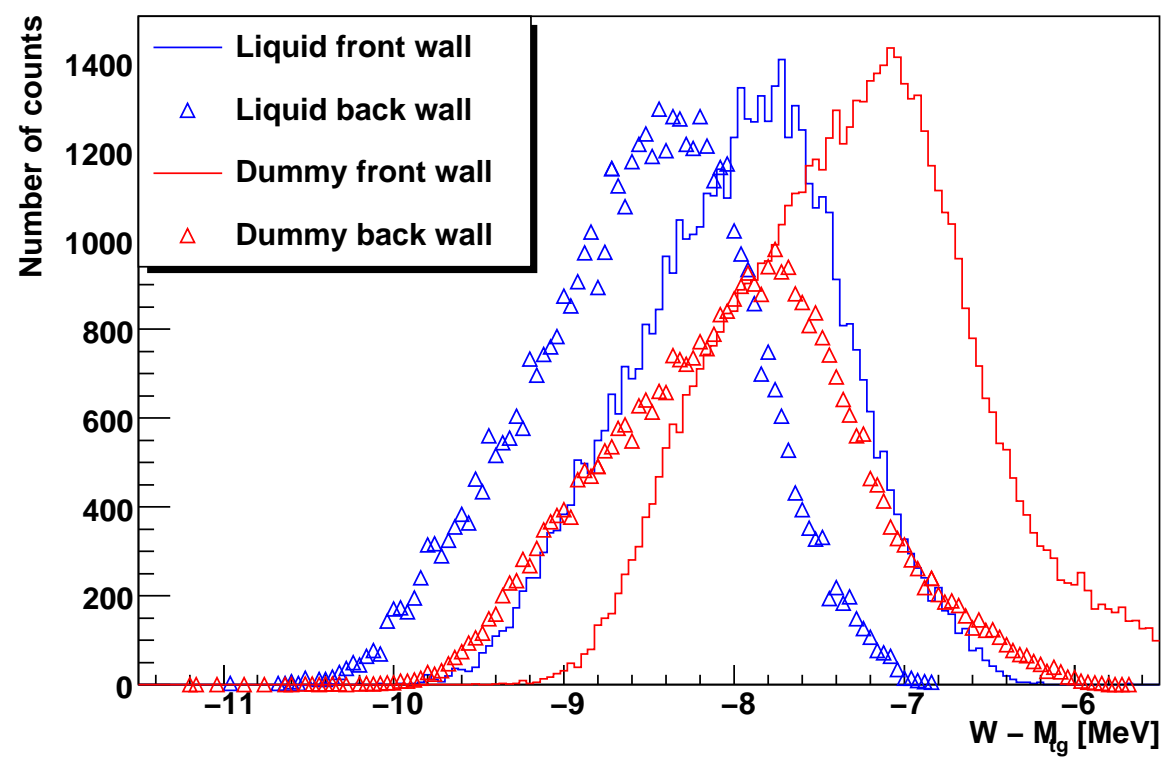

Figure 4.22: The $\mathrm{W}$ distribution of dummy walls and $\mathrm{Al}$. windows at the lowest angle : Four histogram have prominently different distributions.

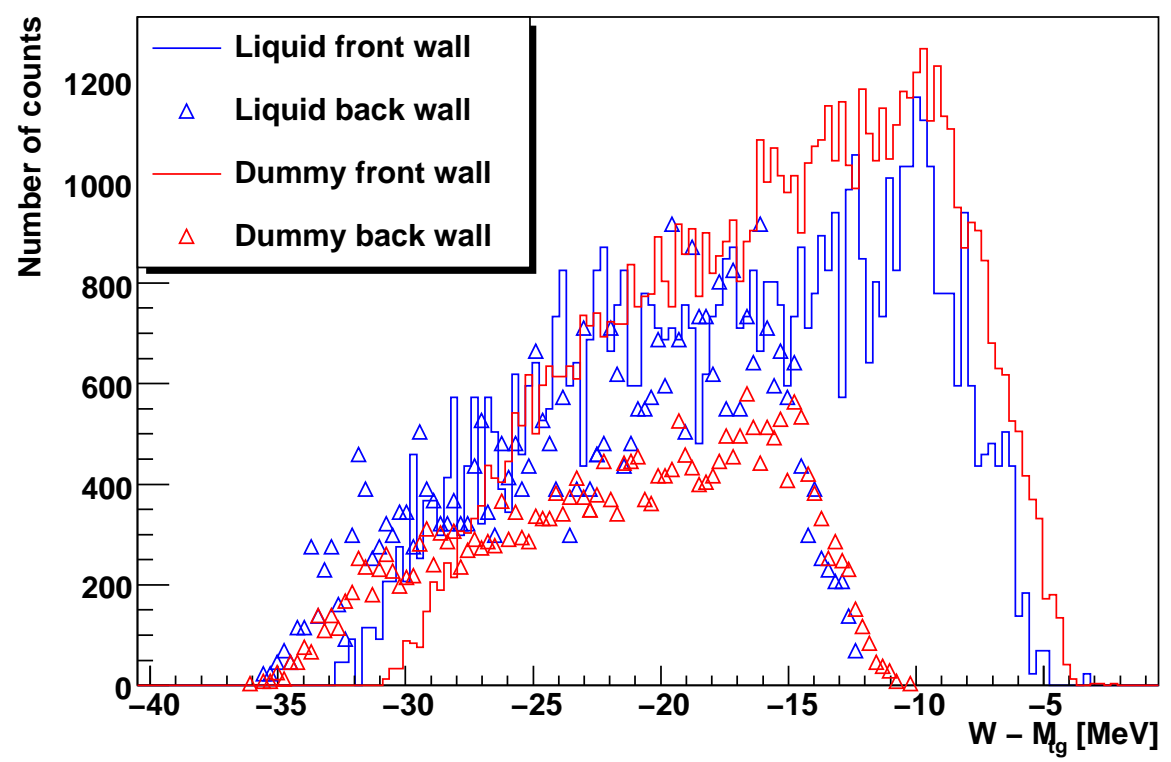

Figure 4.23: The $\mathrm{W}$ distribution of dummy walls and Al. windows at the highest angle : Four histogram have prominently different distributions. 


\subsubsection{Fitting for elastic peak}

The width of an elastic peak in $W$ distribution is different for each kinematics. Moreover the width could be different in a kinematics because some target variables are changing run by run. But variations over runs are much smaller than ones over kinematics. To choose the elastic peak region consistently in every run, a standardized criteria is necessary. In this experiment, a fitting over elastic peak is made with a function for every run of ${ }^{1} \mathrm{H},{ }^{2} \mathrm{D}$ and ${ }^{12} \mathrm{C}$, and then a elastic region is chosen from the fitting parameters consistently.

An elastic peak is shaped as Gaussian distribution with the detector resolution basically but it is distorted and shifted by the energy loss and radiation. Because the radiation tail is out of the elastic region, it is not considered for the fitting. So the fit function is chosen as combinations of two Gaussian functions and one Landau function like Eq. 4.6 and Eq. 4.7.

$$
\begin{gathered}
f(x)= \begin{cases}C_{0} \cdot \exp \left(-\frac{1}{2} \cdot\left(\left(x-C_{1}\right) / C_{2}\right)^{2}\right) & \text { for } x \leq C_{1} \\
C_{0} \cdot \exp \left(-\frac{1}{2} \cdot\left(\left(x-C_{1}\right) / C_{3}\right)^{2}\right) & \text { for } C_{1}<x<C_{1}+1.3 C_{3} \\
C^{\prime} \cdot \exp \left(-\frac{1}{2} \cdot\left(\left(x-C_{1}\right) / C_{3}\right)^{2}\right)+L(x) & \text { for } x \geq C_{1}+1.3 C_{3}\end{cases} \\
\text { B.C. }: f\left(C_{1}+1.3 C_{3}-\delta\right)=f\left(C_{1}+1.3 C_{3}\right), \quad \delta \ll 0,
\end{gathered}
$$

where $L(x)$ is the Landau function and $C^{\prime}$ is a constant determined from B.C. The number 1.3 is chosen after several trial and errors.

$$
f(x)= \begin{cases}C_{0} \cdot \exp \left(-\frac{1}{2} \cdot\left(\left(x-C_{1}\right) / C_{2}\right)^{2}\right) & \text { for } x \leq C_{1} \\ C_{0} \cdot \exp \left(-\frac{1}{2} \cdot\left(\left(x-C_{1}\right) / C_{3}\right)^{2}\right) & \text { for } x>C_{1}\end{cases}
$$

Data of deuteron target have events from inelastic interaction. They should be removed by the elastic peak cut which is explained in section 4.5.4. If the elastic peak cut is applied, the elastic peak is chosen in a narrow region where Eq. 4.7 is enough for the fitting. ${ }^{12} \mathrm{C}$ target is a thin foil and the energy loss of beam and 
scattered electron is very small. The contribution of Landau function becomes very small in ${ }^{12} \mathrm{C}$ target. Therefore Eq. 4.6 is used for the fitting of ${ }^{1} \mathrm{H}$ alone and Eq. 4.7 is used for the fitting of ${ }^{2} \mathrm{D}$ and ${ }^{12} \mathrm{C}$.

Fig. 4.24 - Fig. 4.26 show the fitting result for each target at the lowest angle and the highest angle(exception : ${ }^{12} \mathrm{C}$ is drawn at the angle of $45^{\circ}$ instead of the highest angle because there are few events above $45^{\circ}$ ). The vertical line indicates the center position $C_{1}$ of the fit function. The $x$ axis is drawn as $W-M_{\operatorname{tg}}$ in $[\mathrm{MeV}]$ and $\mathrm{Al}$. walls are already subtracted from liquid target.

\subsubsection{Region of elastic peak}

In the ${ }^{2} \mathrm{D}$ distribution, inelastic events are still convoluted with elastic events after Al. windows are subtracted from the distribution of LD2. Using the fact that the threshold of inelastic scattering is $2.22 \mathrm{MeV}$ (Deuteron binding energy) away from the elastic peak, the region of elastic peak containing few inelastic events can be chosen. The elastic peak region cut is chosen to suppress the number of inelastic events to be less than $0.1 \%$ of number of elastic events. In this process, some of events from the elastic peak and radiation tail are convoluted with inelastic events and they are lost by the cut. These are recovered by the radiative corrections using Monte Carlo simulation.

The elastic peak cut is made by the following procedure and it is described in Fig. 4.27 .

1. The $W$ distribution of pure ${ }^{2} \mathrm{D}$ is obtained. (Top left panel in Fig. 4.27)

2. A region of inelastic events is selected and the average value of number of events $N_{1}$ is obtained. (Top right panel)

This assumes that the number of inelastic events does not change steeply in the distribution.

3. A fit over the elastic peak region is made with Eq. 4.7 and constants $C_{1}$ and 

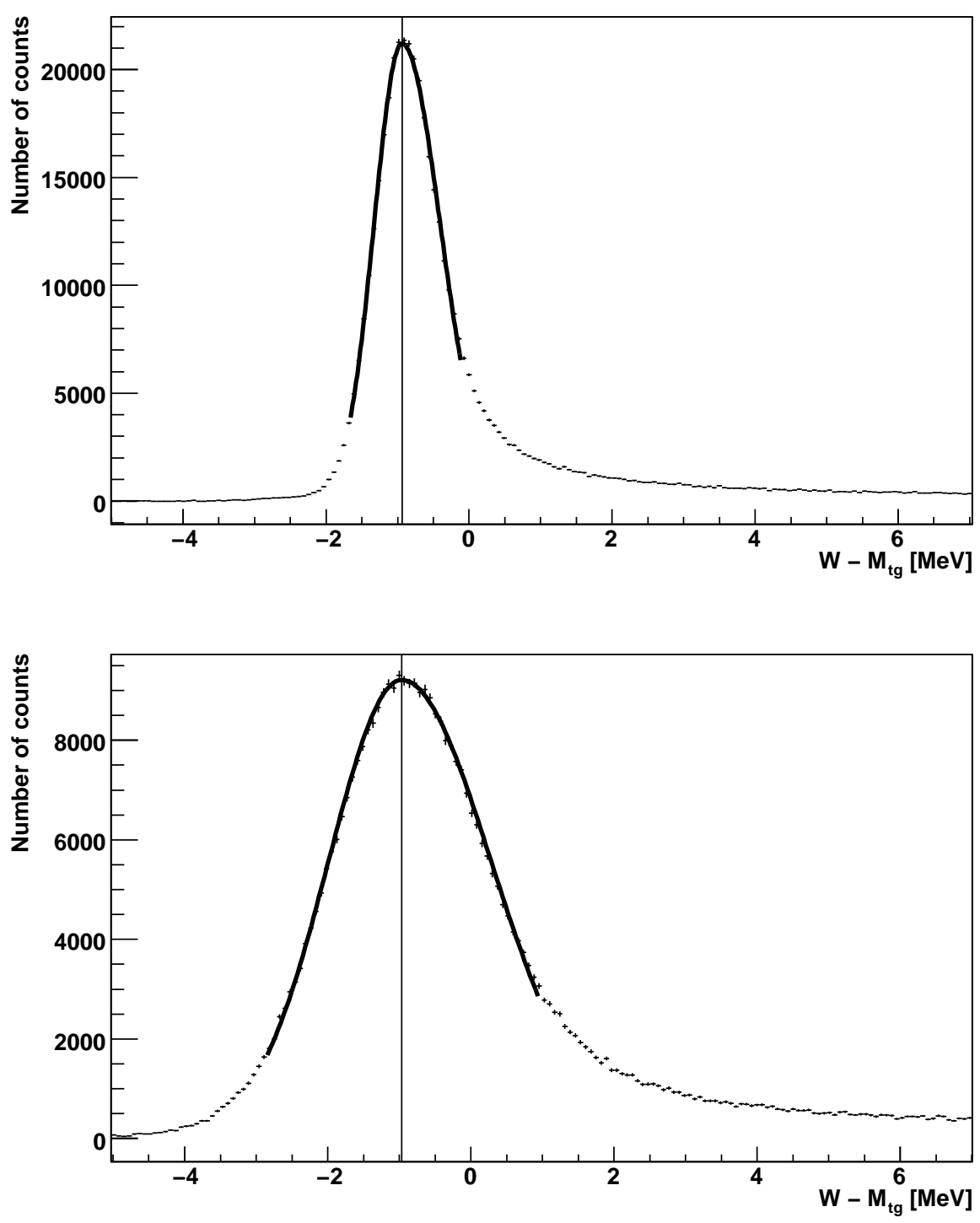

Figure 4.24: Fit over ${ }^{1} \mathrm{H}$ peak. The top plot is at the lowest angle and bottom plot is at the highest angle. The $x$ axis is $W-M_{\mathrm{tg}}[\mathrm{MeV}]$. Al. walls are already subtracted. 

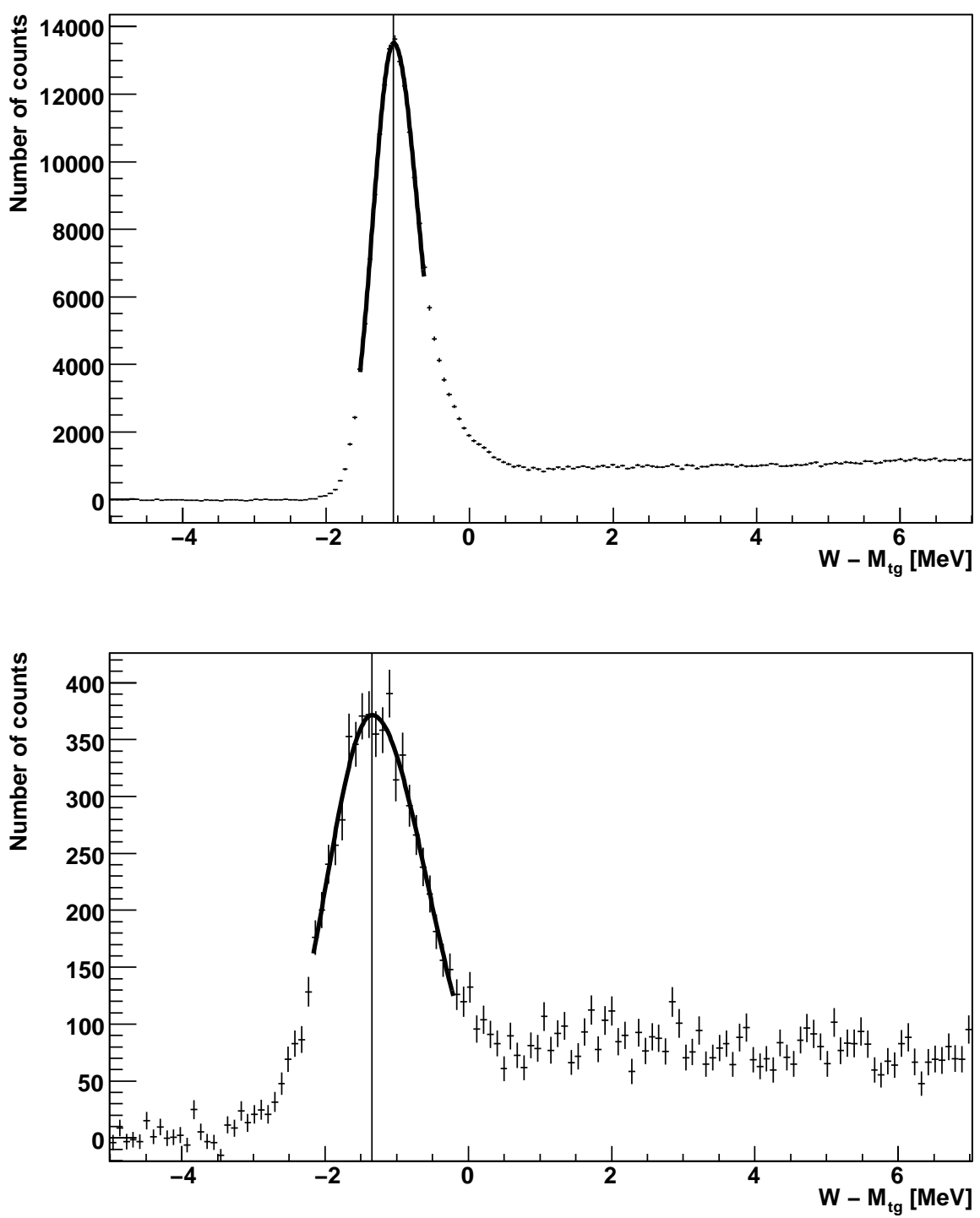

Figure 4.25: Fit over ${ }^{2} \mathrm{D}$ peak. The top plot is at the lowest angle and bottom plot is at the highest angle. The $x$ axis is $W-M_{\mathrm{tg}}[\mathrm{MeV}]$. Inelastic events are seen above elastic peak. Al. walls are already subtracted. 

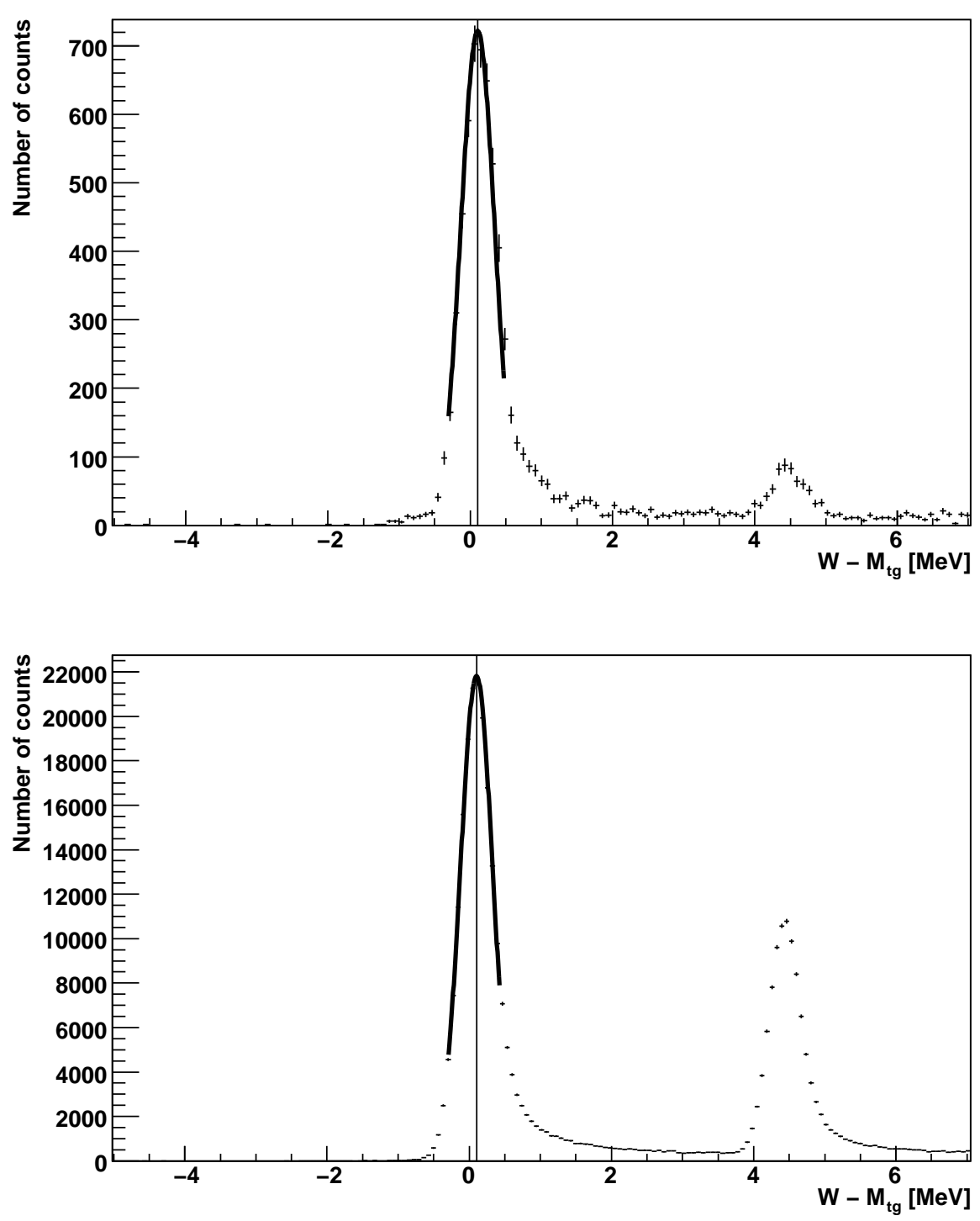

Figure 4.26: Fit over ${ }^{12} \mathrm{C}$ peak. The top plot is at the lowest angle and bottom plot is at the highest angle among available runs. The $x$ axis is $W-M_{\operatorname{tg}}[\mathrm{MeV}]$. The first excited state is shown next to elastic peak. 
$C_{2}$ are found.

$C_{1}$ is the peak center and $C_{2}$ indicates the width of elastic peak. (Middle left panel)

4. Inelastic events with value $N_{1}$ is made using piled up Gaussian functions with width $C_{2}$. The location of the first Gaussian function from the peak center is $2.22 \mathrm{MeV}$.

This curve can be considered as the distribution of inelastic events near elastic peak. And the integral value over the curve is the same with the area of inelastic region selected in process 1.(Middle right panel)

5. The integral value of the piled up function in the elastic peak region is calculated. And then the integral value of the elastic peak function is calculated and the ratio $R$ is defined as the ratio between two integral values. Then a location $W_{\text {cut }}$ is chosen where the ratio $\mathrm{R}$ becomes $0.1 \%$.

6. Finally, the elastic peak cut is chosen from a point below the peak of elastic events to $W_{\text {cut }}$. It is shown as two vertical lines. (Bottom panel)

In bottom panel of Fig. 4.27, the black line is data distribution, the sum of elastic events and inelastic events. The green line is the estimated distribution of inelastic events and the red line is data distribution after the inelastic estimation distribution is subtracted. The region between the two vertical lines is the region of ${ }^{2} \mathrm{D}$ selected by the elastic peak cut.

In large angle kinematics, the width of elastic peak and inelastic events increases and the $W_{\text {cut }}$ approaches to the elastic peak center. Fig. 4.28 shows this phenomena. This is the $W$ distribution at the largest angle. The contribution of inelastic events between two vertical lines is $0.11 \%$ in bottom panel of 4.27 and it is $0.07 \%$ in 4.28 . The elastic region of ${ }^{1} \mathrm{H}$ and ${ }^{12} \mathrm{C}$ are manifest in the $W$ distribution unlike that of deuteron distribution. The ${ }^{1} \mathrm{H}$ distribution has only the elastic peak and radiation tail after Al. windows are subtracted. So no cut is required to choose peak in 

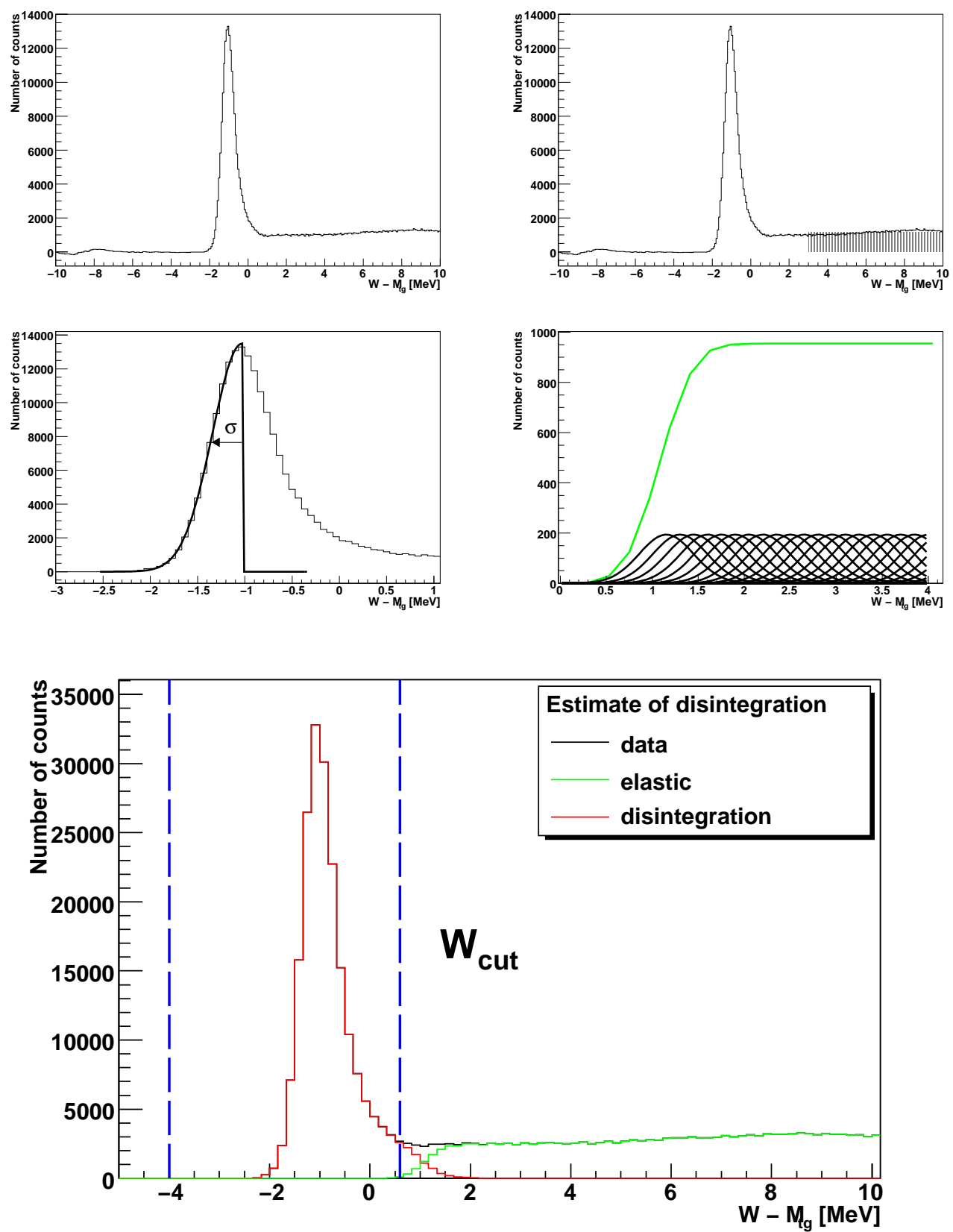

Figure 4.27: Procedure of elastic peak selection in the $W$ distribution of ${ }^{2} \mathrm{D}$. Two vertical lines define the elastic peak region.

Black line : data; Green line : inelastic events ; Red line : elastic data

The distribution of inelastic events are estimated and the contribution is less than $0.1 \%$ of elastic events in the peak region. 


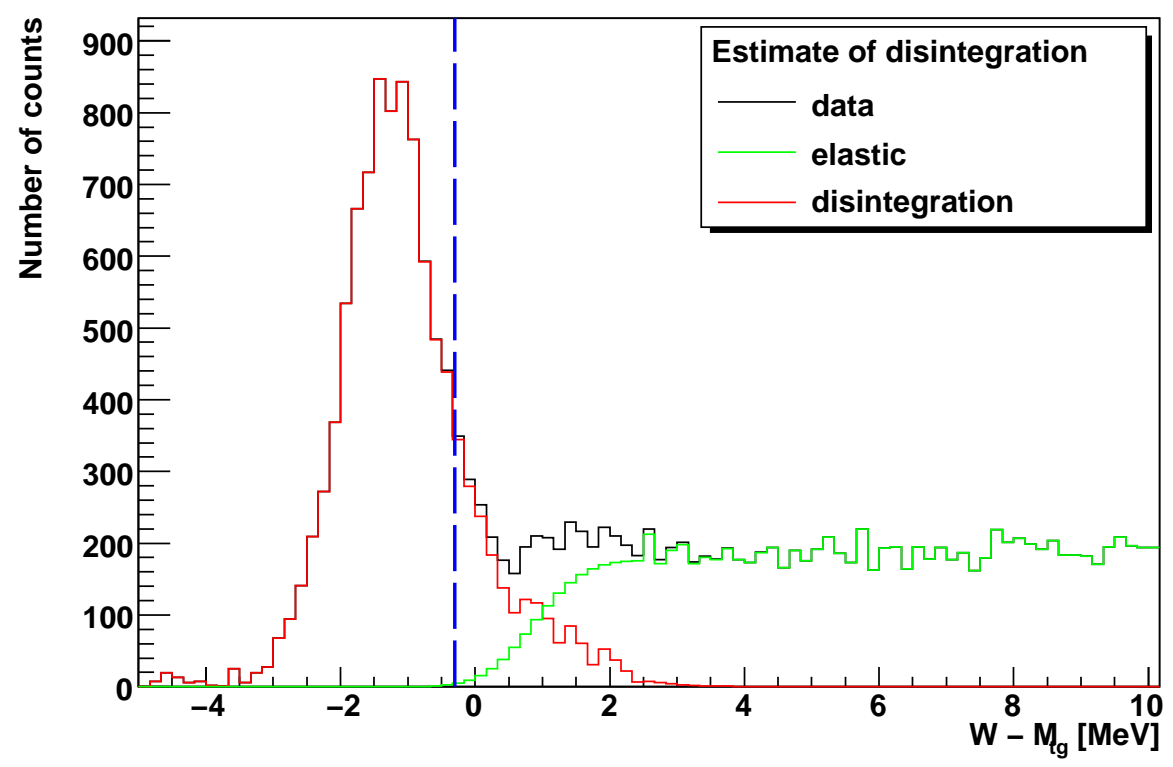

Figure 4.28: Elastic peak cut at the largest angle.

Black line : data; Green line : inelastic events ; Red line : elastic data

$W$ spectrum. But a cut is necessary to suppress the dummy contribution in $Q^{2}$ spectrum. Further explanations about this problem are discussed in section 4.5.5. Hence the elastic cut of ${ }^{1} \mathrm{H}$ is also made in the $W$ distribution to choose a region from a point below elastic peak to $45 \mathrm{MeV}$, a far point from the peak. Fig. 4.29 shows the elastic peak selection for a ${ }^{1} \mathrm{H}$ run.

In the $W$ distribution of ${ }^{12} \mathrm{C}$, the first excited state peak is located $4.44 \mathrm{MeV}$ away from the ground state peak. So the elastic peak cut of ${ }^{12} \mathrm{C}$ is chosen to be $-10 \mathrm{MeV}<$ $W-M_{\mathrm{tg}}<3.0 \mathrm{MeV}$. Fig. 4.30 shows the elastic peak selection for a ${ }^{12} \mathrm{C}$ run. Lost events above $3.0 \mathrm{MeV}$ are compensated by the Monte Carlo simulation with radiative corrections. 


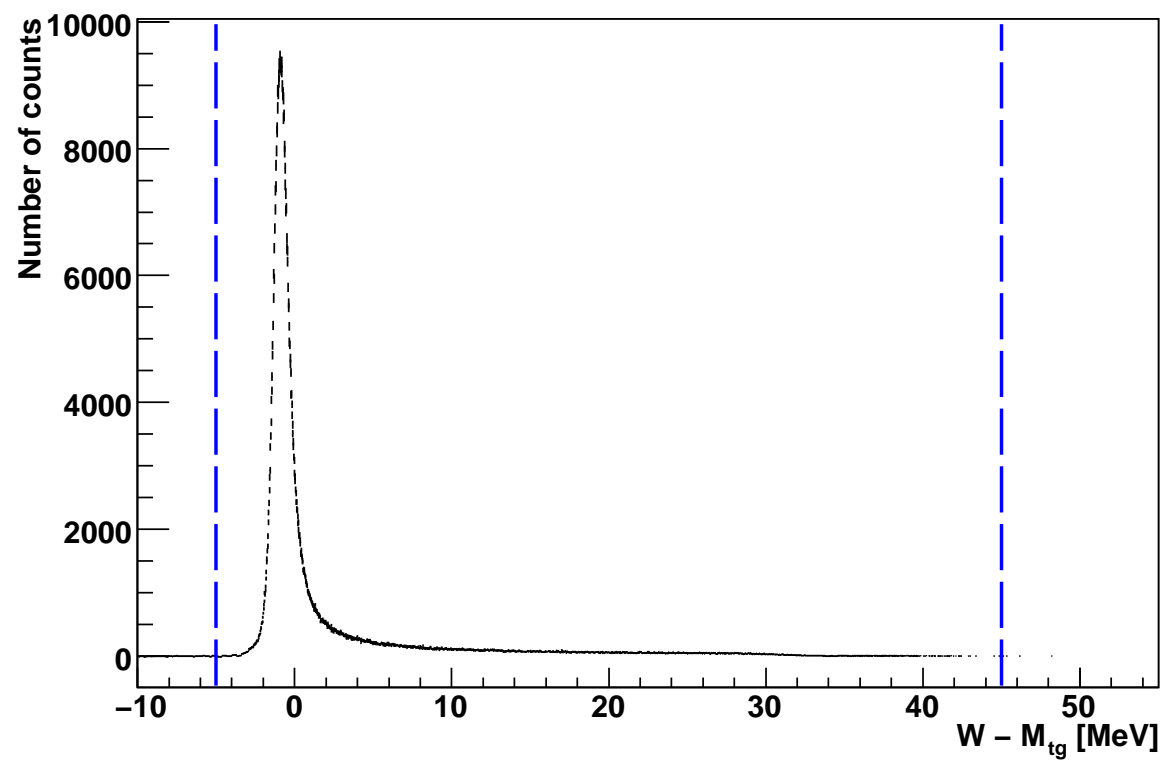

Figure 4.29: The elastic peak region selection for ${ }^{1} \mathrm{H}$ target.

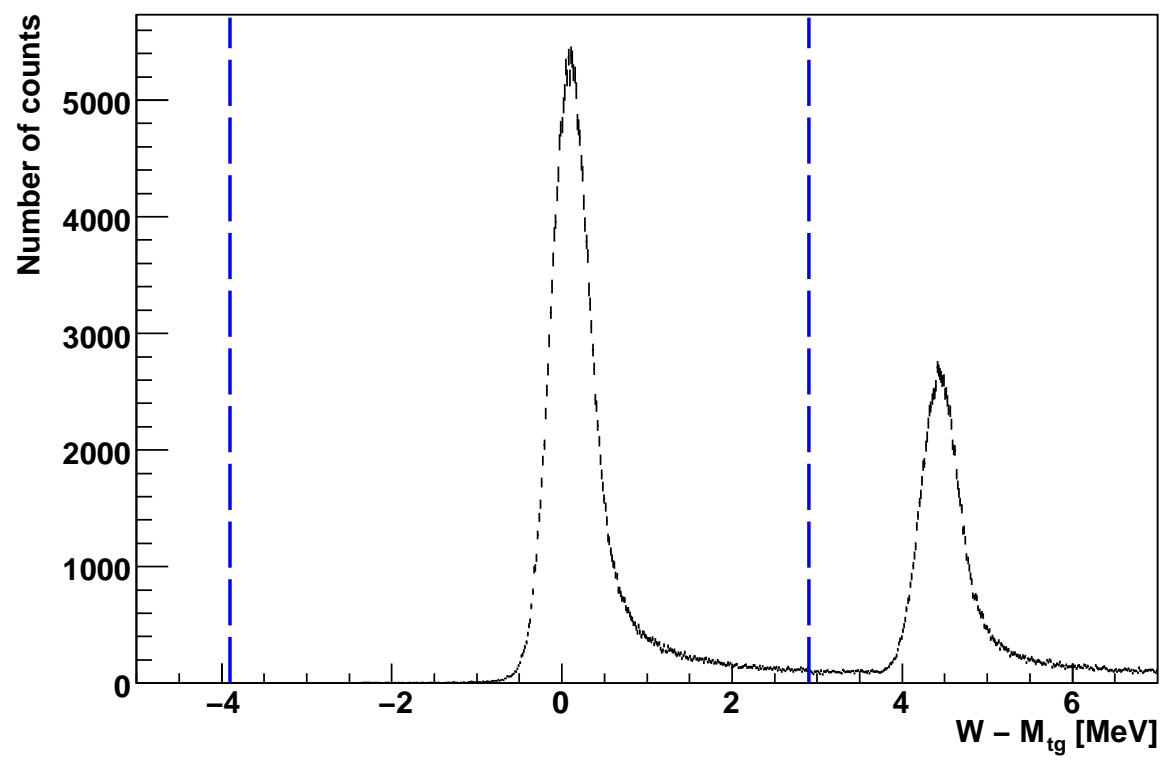

Figure 4.30: The elastic peak region selection for ${ }^{12} \mathrm{C}$ target. Events from the first excited state are excluded. 


\subsubsection{End cap subtraction in $Q^{2}$ spectrum}

To obtain the cross section $\frac{d \sigma}{d \Omega}\left(Q^{2}\right)$, the distribution of $Q^{2}$ is required. Obviously $Q^{2}$ distribution is defined by electrons only and it is not closely related with target mass whereas $W$ is sensitive on the change of target mass. Events from Al. windows are entangled with events from pure ${ }^{1} \mathrm{H}$ and ${ }^{2} \mathrm{D}$ in $Q^{2}$ distribution.

In section 4.5.2, a weight function $\omega(W)$ is introduced to subtract contributions of $\mathrm{Al}$. windows in $W$ distribution. If $\mathrm{Al}$. windows and pure target of ${ }^{1} \mathrm{H}$ or ${ }^{2} \mathrm{D}$ have the same $Q^{2}$ distribution, $\omega(W)$ can be used for the end cap subtraction in $Q^{2}$ too. But if two of them have quite different distributions, some uncertainties can be generated. $(\omega(W)$ does not care whether an event comes from Al. windows or pure target of ${ }^{1} H$ or ${ }^{2} D$. Consequently it will count each event as $\omega(W)$ by the value of $W$ regardless of the $Q$ value.) So $Q^{2}$ distributions of two Al. windows and ${ }^{2} D$ are compared in Fig. 4.31. ${ }^{10}$ The top panel is drawn at the lowest angle and the bottom panel is drawn at the highest angle.

The total number of all distributions are normalized for comparison. The red line indicates events from front Al. window and the green line indicates events from back Al. window. The blue line indicates events from ${ }^{2} D$.

Big difference is shown between three distributions. And the distribution of front Al. window is shifted from the one of back Al. window. It is prominent that the assumption to use $\omega(W)$ for $Q^{2}$ distribution is wrong. And a new weight function $\omega\left(Q^{2}\right)$ should be made for the end cap subtraction in the $Q^{2}$ distribution.

The method to calculate $\omega\left(Q^{2}\right)$ is similar with the one for $\omega(W)$ except that the normalization factor of dummy walls is already calculated in the process of $\omega(W)$ so it can be used again.

Functions $\omega(W)$ and $\omega\left(Q^{2}\right)$ do not generate big difference at low angle kinematics because contributions of $\mathrm{Al}$. windows are small. On the other hand the difference

\footnotetext{
${ }^{10}$ Events from two Al. windows or ${ }^{2} D$ are separated by the position of $W_{\mathrm{Al}}$. and reaction z. In this case, some amount of contribution of Al. windows are contained in $Q^{2}$ distribution from ${ }^{2} D$ but it is negligible.
} 

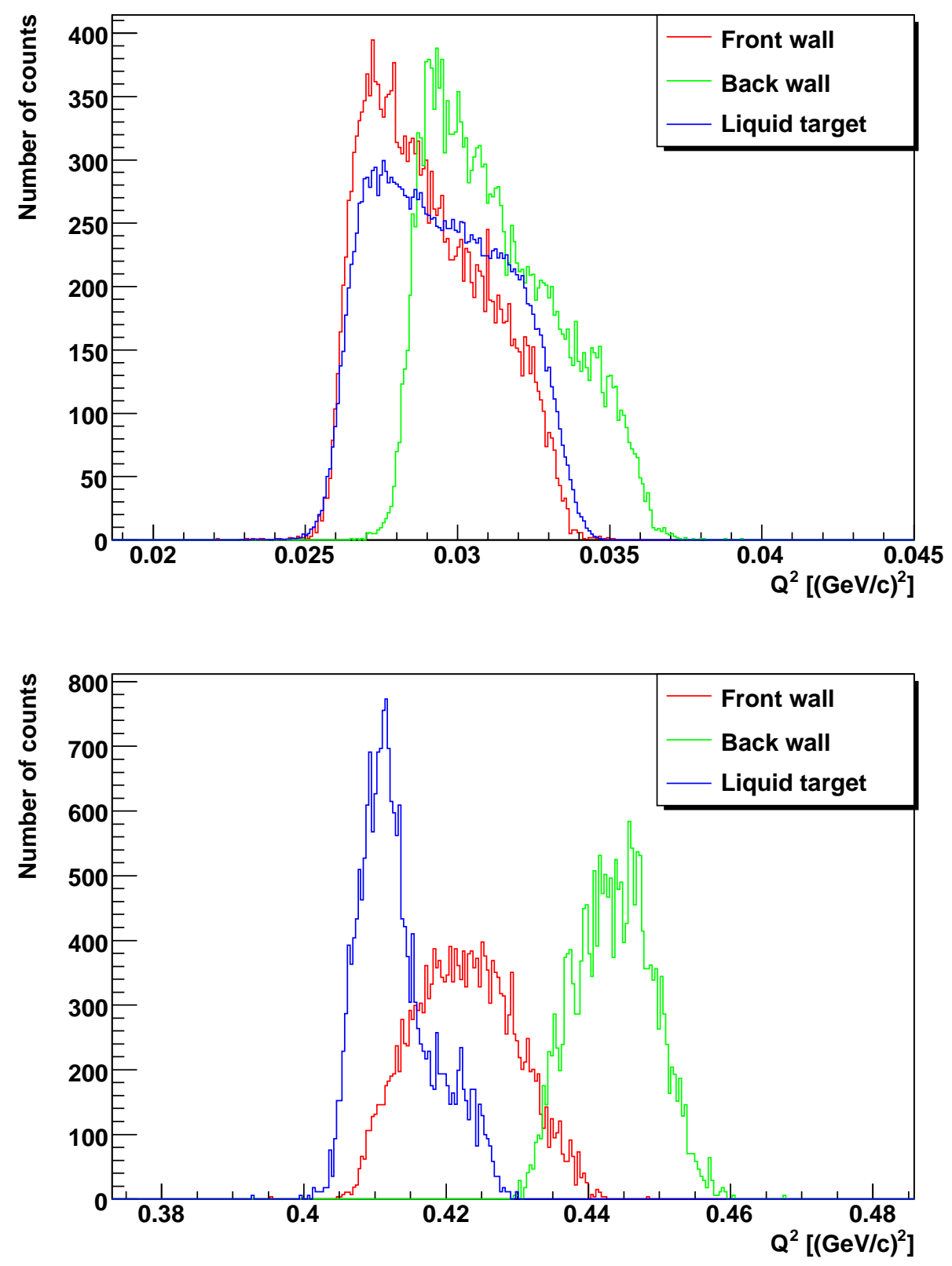

Figure 4.31: $Q^{2}$ distributions of two Al. windows and pure ${ }^{2} D$. Their shapes are quite different. 
between two methods exceeds several \% in large angle kinematics of LD2 where contributions of Al. windows are big. Fig. 4.32 compares two $Q^{2}$ distributions made by $\omega\left(Q^{2}\right)$ and $\omega(W)$ with the red line and black one in top panel. The calculation by $\omega(W)$ underestimates contributions of $\mathrm{Al}$. windows in the center region and overestimates in the out region. The ratio between them is drawn in the bottom panel. It is drawn as percentage over $Q^{2}$. It shows that the method misusing $\omega(W)$ instead of $\omega\left(Q^{2}\right)$ could give several \% systematic error in the meaningful center region.

\subsection{Monte Calro Simulation}

The yield of elastic scattering in $W$ distribution can be defined with the number of events in the regions of elastic peak. The elastic peak is defined by the elastic peak cut explained in section 4.5.4. But some events are lost in the yield calculation and there are two cases.

- Some events lost their energy in the passage by radiation and they build up the radiation tail from elastic peak. They are moved out of the elastic peak. However they are originally elastic events and they should be counted for the elastic yield.

- The elastic events are convoluted with inelastic events in deuteron scattering. Elastic peak cut is originally constructed to suppress the contributions of inelastic events. In this process, some of elastic events are lost inevitably. These missing events can be reconstructed by the Monte Carlo simulation. Details of simulation process is explained in this section.

\subsubsection{MCEEP}

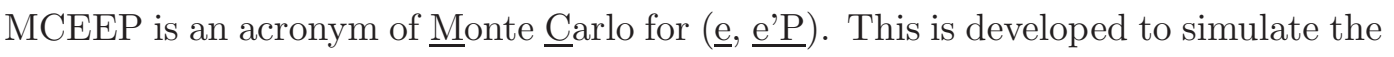
scattering process in JLab Hall A environment. [52] MCEEP can simulate several 

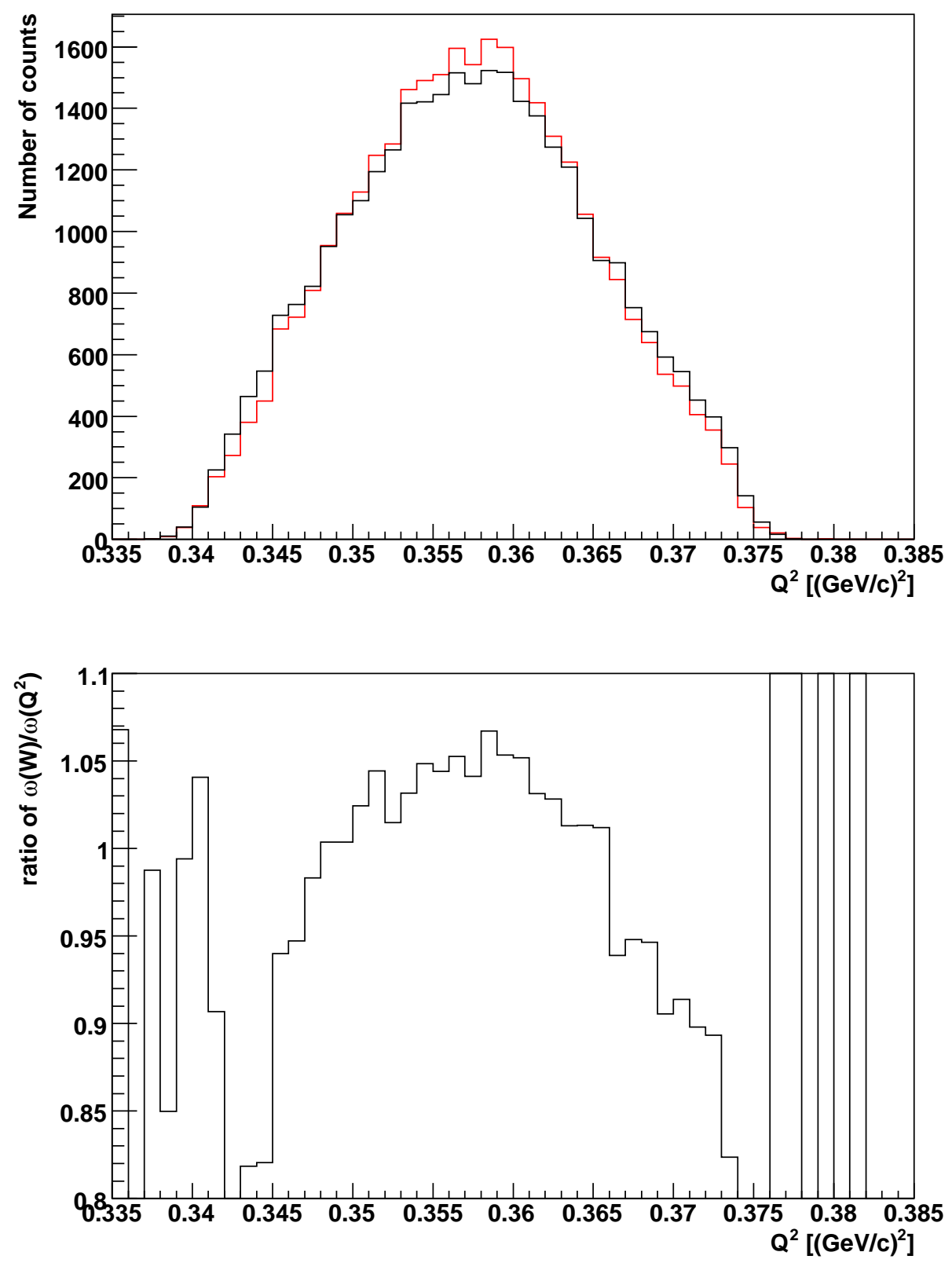

Figure 4.32: Top panel : $Q^{2}$ distributions made by two methods. Red line : $\omega\left(Q^{2}\right)$; Black line $\omega(W)$ Bottom panel : The ratio distribution of two functions. $\omega(W)$ could give a few $\%$ error. 
scattering types such as elastic scattering and inelastic scattering of bound states and continuum residual system. It simulates the scattering process based on the input variables such as beam, target, kinematic setting, HRS and final state. For example, the beam injection angle, beam energy, target mass, scattering angle, HRS momentum setting, size of detector entrance, HRS mispointing can be given as input variables.

The concept of MCEEP process is given below.

- Events within the acceptance range of momentum and solid angle are chosen at target.

- The predefined interaction is applied by the kinematic setting.

- Events are transported into the spectrometer and then acceptance cuts are applied.

- Resolution smearing effects from detectors are added.

- The cross section is calculated for each event by its kinematic variables. The interpolation, extrapolation and fit function from old data are used for cross sections.

- The calculated cross sections are applied as weight factor to whole events.

This is simplified concept but the real calculation of MCEEP is more complex. Whole simulation process is fully explained in MCEEP manual book. The previous procedure is slightly different with the true Monte Carlo simulation which generates all events with predefined weight. But this method requires smaller number of events and it is efficient for simulating the Hall A scattering environment.

MCEEP can simulate the effects of radiation tail and detector resolution also. In this analysis, MCEEP is used to calculate the radiative correction, acceptance and systematic error of kinematics. 
In this analysis, elastic scattering of three $\operatorname{targets}\left({ }^{1} \mathrm{H},{ }^{2} \mathrm{D},{ }^{12} \mathrm{C}\right)$ are simulated by MCEEP. However the cross section of specific interaction is required to simulate a scattering process. Cross sections of ${ }^{1} \mathrm{H}$ and ${ }^{12} \mathrm{C}$ are already well known from the world data and several fit functions exist. Two fit functions $[54,53]$ are given to MCEEP to simulate the elastic scattering with ${ }^{1} H$ and ${ }^{12} C$ in the kinematics of this experiment.

The radiative correction and acceptance of ${ }^{2} \mathrm{D}$ are calculated from MCEEP. For cross section of ${ }^{2} \mathrm{D}$, a fit function from world data [4] is used. ${ }^{11}$

\subsubsection{Radiation in MCEEP}

Though the electromagnetic cross section is defined at one photon exchange interaction in the born approximation, detected electrons are always modified by radiations. Hence experimental data values are already dressed by the radiation effects and no method exists to measure bare data directly. Instead, the amount of radiation effects can be calculated and it can be applied to experimental data as a correction efficiency. MCEEP has implemented radiation calculation algorithms and it resolves the radiation problem.

In the process of electron and nucleus interaction, there are two contributions from internal radiation and external radiation. The internal radiation is originated from interacting particles themselves during the interaction $\left(e, e^{\prime} N\right)$. On the other hand, the external radiation is generated from other target nucleus. This includes the multiple scattering and the energy loss either.

\section{Internal radiation}

In an experiment, there exists a low energy limit(cutoff) generated from the detector resolution. Photons whose energy is smaller than the energy limit can be treated as soft photon. MCEEP considers the internal radiation with two parts. One is from

\footnotetext{
${ }^{11}$ Approximate values of cross section are enough in the MCEEP usage for ${ }^{2} \mathrm{D}$.
} 
the virtual photon and the soft photon. These are described by Schwinger term [55] and the treatment is taken from Penner calculation [56]. Another is from the real photon which is not soft. This is the Bremsstrahlung interaction and it generates the radiation tail of elastic peak. The peaking approximation method of Mo and Tsai [57] are used for the radiation tail. While photons can be emitted into any direction during the Bremsstrahlung process, the peaking approximation assumes that photons are radiated along the incident direction or the scattered electron direction. The cutoff value is chosen as $0.35 \mathrm{MeV}$ to define the soft photon.

\section{External radiation}

There are several reactions that can cause external radiation. MCEEP counts four cases

- Electron energy loss : When charged particles pass through a matter, they suffer the energy loss and spread of distribution. The mean energy loss of electrons is obtained from the Bethe-Bloch formula [58].

The beam energy and scattered electron energy are corrected by this formula. The energy loss is smaller than $1 \mathrm{MeV}$ in this experiment because only foil target and $4 \mathrm{~cm}$ short extended targets are used.

- Straggling: The spread of distribution, straggling, is described by three distribution functions of Landau, Vavilov and Gaussian [47].

- Multiple scattering : If an electron is scattered with a small angle at first, it could have another chance of multiple scattering. MCEEP uses the Moliere scattering formula [59] for multiple scattering.

- External Bremmstrahlung : Injecting electrons are affected by the Coulomb field of other nucleus and undergo interaction. These effects are treated by Bremmstrahlung interaction function [47]. 


\subsection{Radiative Corrections}

The radiation effects in cross section calculation for carbon, hydrogen and deuterium targets are calculated from MCEEP simulations. MCEEP applies all kinematic setting of this experiment by the given input file and it calculates distributions of every target variable twice, one with the radiation effect and another without the effect.

If these two distributions are compared, the change of yield by radition can be calculated. It can be applied to the deuteron target either. In the $W$ distribution of ${ }^{2} \mathrm{D}$, some elastic events are lost by the elastic peak cut. If MCEEP simulates the deuteron elastic scattering, the number of lost events can be predicted. (The ratio of two simulation distributions before and after applying the elastic peak cut.) Only the ratio of change is necessary, so values of deuteron cross section used in MCEEP calculation do not need to be accurate.

As seen in section 1.2, the scattered electron energy $E^{\prime}$ can be determined from two other kinematic variables such as beam energy $E$ and scattering angle $\theta$ in the elastic scattering. And the $\frac{d \sigma}{d \Omega}\left(Q^{2}\right)$ is a meaningful differential cross section. Hence distributions of $Q^{2}$ is necessary to obtain cross sections and radiative correction also should be calculated from $Q^{2}$ distribution. The correction is processed by below procedure.

1. A $Q^{2}$ distribution histogram $h_{\mathrm{Mwo}}$ is made from MCEEP without radiation effects.

2. A $Q^{2}$ distribution histogram $h_{\mathrm{Mw}}$ is made from MCEEP with radiation effects. This should have the same bin setting with $h_{\mathrm{Mwo}}$.

3. Values of histogram $h_{\mathrm{Mwo}}$ at each bin are divided by values of histogram $h_{\mathrm{Mw}}$

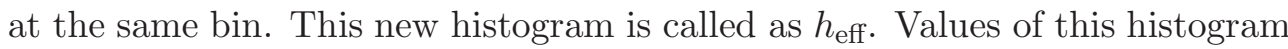
at each bin is the efficiency of radiative correction at each point of $Q^{2}$. 
4. A $Q^{2}$ distribution histogram $h_{D}$ is drawn from experimental data.

5. Values of histogram $h_{D}$ at each bin is multiplied by correction values of histogram $h_{\text {eff }}$ at the same bin. This process can be written by eq.4.8.

$$
\mathrm{DATA}_{\text {corrected }}=\mathrm{DATA}_{\text {uncorrected }} \times \frac{\mathrm{MCEEP}_{\text {corrected }}}{\mathrm{MCEEP}_{\text {uncorrected }}}
$$

Elastic peak cuts obtained in section 4.5.4 are applied first for all histograms except $h_{\text {Mwo }}{ }^{12}$. Consequently both correction effects from not the radiation and elastic peak cut are included in $h_{\text {eff }}$.

Above procedures for a carbon run are drawn in Fig. 4.33. Several distributions of $Q^{2}$ are drawn. Experimental data are shown as red line and MCEEP simulation results are shown as black line. The top left panel is the $Q^{2}$ distribution of data elastic events. The top right panel is the $Q^{2}$ distribution of simulation. The middle left panel is the $Q^{2}$ simulation without the radiation effect and elastic peak cut. The middle right panel is the ratio of two plots in the middle left and top right. It is the histogram of efficiency(radiative correction and elastic peak cut). New data with radiative correction is drawn in bottom panel and it is obtained from multiplications of two plots in top Left and mid right.

Fig. 4.34 compares $Q^{2}$ distributions of ${ }^{1} \mathrm{H}$ and ${ }^{2} \mathrm{D}$ before and after the radiative correction. The effect of elastic peak cut is also included. The black line is before the calibration and the red line is the corrected one. The top panel is from a ${ }^{1} \mathrm{H}$ run and the bottom panel is from a ${ }^{2} \mathrm{D}$ run. The recovery of ${ }^{2} \mathrm{D}$ is bigger since the elastic peak cut of ${ }^{2} \mathrm{D}$ is narrower.

\footnotetext{
${ }^{12}$ The elastic peak cut is defined on the distribution of $W$. So the $W$ distribution is made in MCEEP also. Then elastic peak fitting is executed for the MCEEP histogram with the same method for data, since the position of peak in MCEEP could be shifted from one of data histogram.
} 

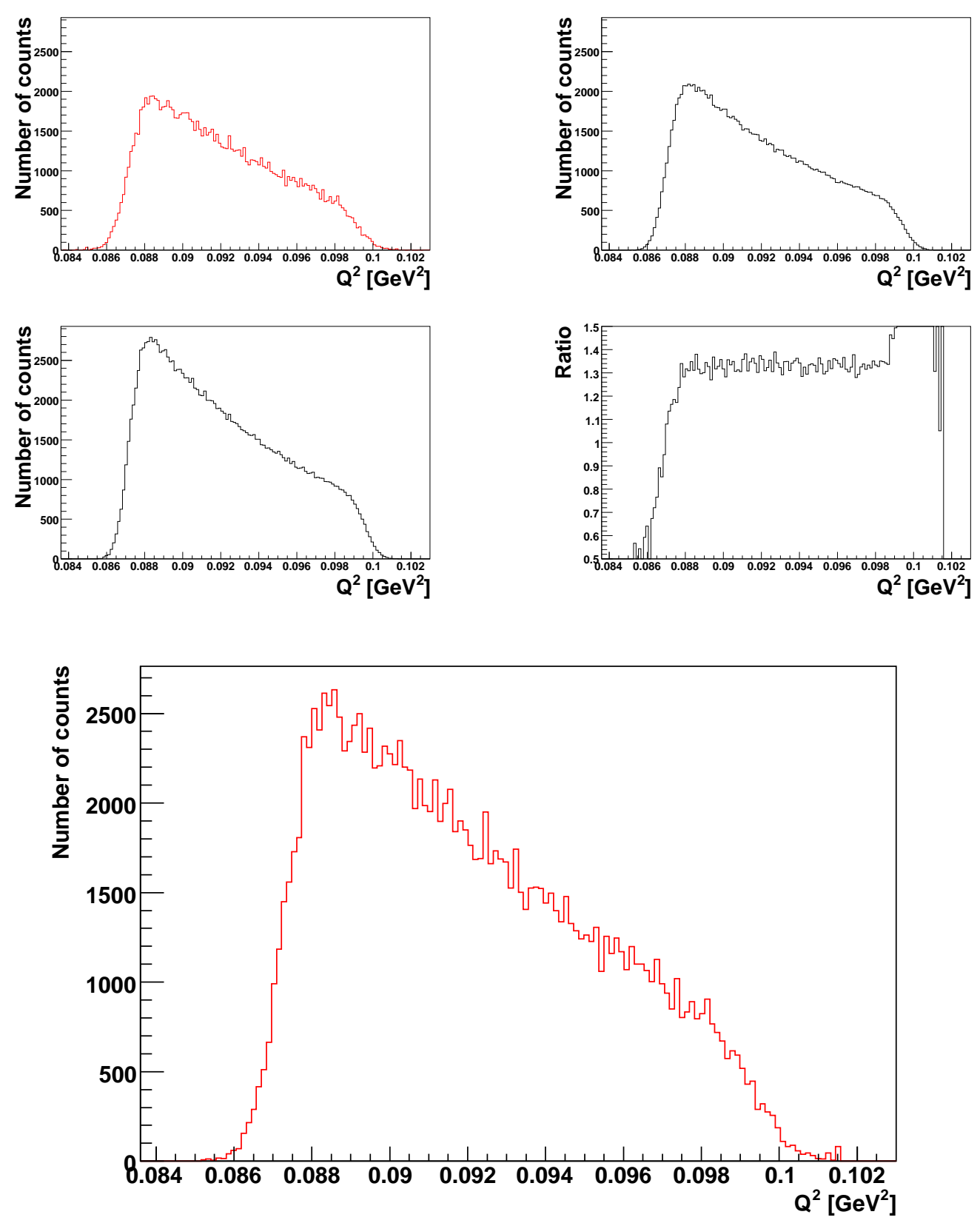

Figure 4.33: Radiative corrections.

Red line : Experimental data ; Black line : MCEEP simulation.

Top panels are $Q^{2}$ distributions of data(only elastic events) and $Q^{2}$ simulation. Middle panels are $Q^{2}$ simulation without the radiation effect and the efficiency histogram of radiative correction. The bottom panel shows the data distribution after the radiative correction is applied. 

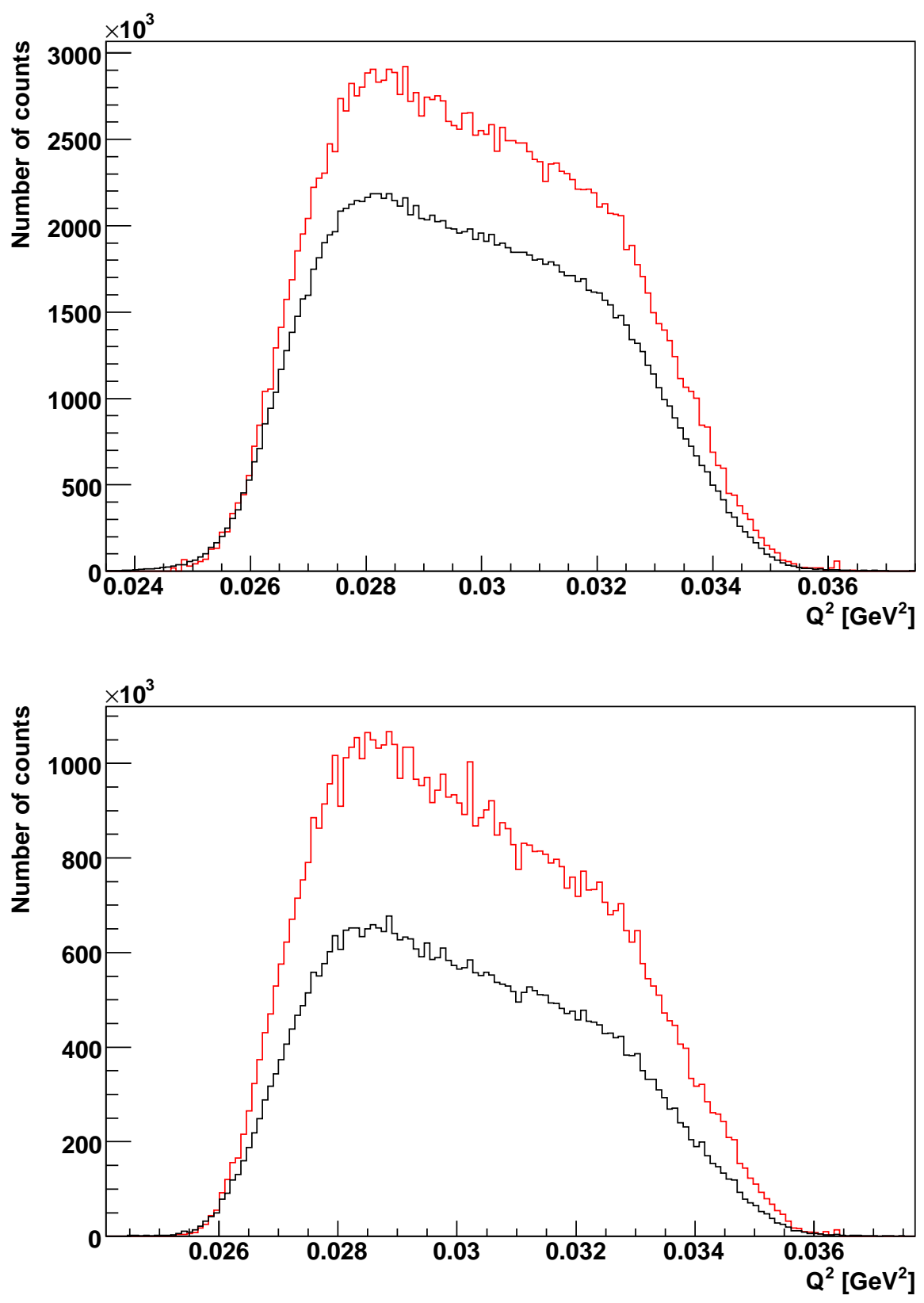

Figure 4.34: $Q^{2}$ distributions of ${ }^{1} H$ (Top panel) and ${ }^{2} D$ (Bottom panel) after the radiative correction is applied.

Black line : Before correction ; Red line : After corrected.

The recovery of deuteron is bigger because the elastic peak cut in ${ }^{2} D$ is narrower. 


\subsection{Systematic Uncertainties}

Deuteron cross sections are obtained as the ratio to hydrogen cross sections ${ }^{13}$. In all kinematics, a hydrogen run is measured first and then a deuteron run is measured without any change of experimental setting. Some of kinematic variables and normalization factors are the same between two runs of hydrogen and deuteron. In this case, some of systematic errors are cancelled or values are reduced. But in some cases, random errors become larger because the error from hydrogen is added. There are several types of systematic errors such as kinematic errors, normalization errors and event selection errors.

Kinematic errors are caused by the wrong information of kinematic variables. The variable that can cause the biggest error is the scattering angle and beam energy. In a kinematics, the scattering angle does not change and runs of deuteron and hydrogen have the same angle. So the importance of angle information is reduced in ratio method. It is similar for the beam energy if there is no abrupt change between two target runs. It is checked for the scattering angle with uncertainty of $0.3 \mathrm{mrad}$ and the beam energy with uncertainty of $0.2 \%$. Because of the cancellation, they give negligible errors.

Normalization errors are generated in the process obtaining normalization factors. The error of target density becomes larger in the ratio method because two random errors for hydrogen and deuteron are considered. On the other hand the beam current can be cancelled in some kinematics if runs of hydrogen and deuteron have uniform beam current. The biggest normalization error is coming from the beam current of $0.5 \%$.

Liquid target cells have curvature at back side and it generates the effective change of target length by the different beam position. So the error of target length correction is the error generated from the correction job. The calculated value is $0.2 \%$. If the ratio of deuteron cross sections to hydrogen cross sections is obtained, it should

\footnotetext{
${ }^{13} \mathrm{It}$ is described in sectioin 5.4 and 5.5 .
} 
Table 4.5: Systematic Errors

\begin{tabular}{|c|c|}
\hline Quantity & Uncertainty [\%] \\
\hline Kinematic error & - \\
Efficiencies & 0.2 \\
Beam Charge & 0.5 \\
Target Length & 0.1 \\
Correction & \\
Target Boiling & 0.4 \\
Target Density & 0.2 \\
Yield extraction & 0.6 \\
${ }^{1} \mathrm{H}$ fit ratio & 1.0 \\
Subtraction & - \\
Radiative Correction & 0.1 \\
Acceptance & 0.8 \\
\hline Overall systematic & 1.6 \\
\hline
\end{tabular}

be multiplied to the standard hydrogen data. In this analysis, a fit function from hydrogen world data [53] is chosen as the standard of hydrogen data. The fit function has its own error of fitting and it also should be considered. It is known as $1 \%$ and the biggest systematic error component.

The last type of error is coming from the yield extraction. Several cases correspond to this. The biggest error is coming from elastic events selection. The fitting over elastic peak region has error. And the event selection is made at the $W$ histogram and there is the error of the bin size. The total error of $0.6 \%$ is predicted in the yield extraction process. All components of systematic errors are in Table 4.5. 


\section{Chapter 5}

\section{Cross sections of Carbon and Hydrogen}

\subsection{Overview}

In previous chapters of data analysis, the range of elastic peak is chosen and then the number of elastic events for each run is obtained. All factors of normalization and corrections are also obtained. From these two terms, the elastic yield of each run is extracted. In addition, the acceptance is obtained from the MCEEP calculation. If the yield is divided by the acceptance, the cross section of each run can be obtained. In section 1.2, it is explained that the cross section of an electron interacting with a nucleus is divided into two terms of non-structure cross $\operatorname{section}\left(\left.\frac{\mathrm{d} \sigma}{\mathrm{d} \Omega}\right|_{\mathrm{NS}}\right)$ and form factor $\left(\left|F\left(Q^{2}\right)\right|^{2}\right)$. $\left.\frac{\mathrm{d} \sigma}{\mathrm{d} \Omega}\right|_{\mathrm{NS}}$ is pure kinematic term and $\left|F\left(Q^{2}\right)\right|^{2}$ describes the inner structure of the nucleus. Because the kinematic setting of each experiment differs, $\left|F\left(Q^{2}\right)\right|^{2}$ is better than $\frac{\mathrm{d} \sigma}{\mathrm{d} \Omega}$ to observe the discrepancies among experimental data. Experimental cross sections and values of $F\left(Q^{2}\right)$ are obtained with below procedure for targets of ${ }^{12} \mathrm{C},{ }^{1} \mathrm{H}$ and ${ }^{2} \mathrm{D}$.

1. The number of elastic peak is obtained. 
2. Normalization factors such as beam current calibration and target density correction are obtained.

3. Correction factors such as multi-track efficiency and radiative correction are applied and yield is extracted.

4. The acceptance is obtained.

5. The cross section values are obtained.

6. The Form factor $\left|F\left(Q^{2}\right)\right|^{2}$ values are obtained from $\frac{\mathrm{d} \sigma}{\mathrm{d} \Omega} /\left.\frac{\mathrm{d} \sigma}{\mathrm{d} \Omega}\right|_{\mathrm{NS}}$

Especially, values of $F\left(Q^{2}\right)$ are compared with extracted values from the world data fit for targets of ${ }^{12} \mathrm{C},{ }^{1} \mathrm{H}$. Systematics of this experiment are checked from the comparison. Results of ${ }^{12} \mathrm{C}$ and ${ }^{1} \mathrm{H}$ measurements are described.

\subsection{Acceptance}

The acceptance distribution over $Q^{2}$ is required to extract the cross section over $Q^{2}$. The angular acceptance is defined by the collimator attached at the entrance of spectrometer. However the momentum acceptance range and target length complicates the calculation of acceptance. In addition, trajectories of scattered electrons are modified and some electrons are lost through spectrometer passage. Hence the angular acceptance is calculated by the Monte Carlo simulation. MCEEP implements several algorithms to obtain the acceptance and specific method can be chosen by the input option. Especially the SNAKE $[60,36]$ method is adapted in this analysis. SNAKE is a ray-tracing code and it can trace trajectories of electrons through magnets and apertures in spectrometer. 


\section{3 $\quad$ Yield in $Q^{2}$ Spectra}

The scattering yield histogram in $Q^{2}$ spectra for each kinematics is obtained and it is compared with the MCEEP simulation histogram. MCEEP includes all characteristics of HRS such like the acceptance and the collimator size. The carbon elastic scattering is generated with cross sections from a world data fit function [54]. Hence if yields from both histograms agree well, it means that cross sections of this experiment agree well with the fit function of world data and the acceptance from MCEEP describes well HRS settings of this experiment.

Fig. 5.1 - Fig. 5.8 show scattering yields of ${ }^{12} \mathrm{C}$ data. Left panels are distributions of $Q^{2}$ and right panels are distributions of $\left(P-P_{0}\right) / P_{0}$, the momentum deviation ratio from the central momentum setting $P_{0}$. Data are marked with red symbols and simulation results are shown as black curve. Carbon has several excited states and they are shown in data distributions. But they are not seen in simulation distributions which have only ground state. For kinematics whose angles are larger than $50^{\circ}$, cross sections are very low and few events are detected. They are not drawn here.

Fig. 5.9 - Fig. 5.20 show yield distributions of ${ }^{1} \mathrm{H}$ data. Left panels are distributions of $Q^{2}$ and right panels are distributions of $\left(P-P_{0}\right) / P_{0}$ like the case of ${ }^{12} \mathrm{C}$. The elastic scattering of hydrogen is simulated by a world ${ }^{1} \mathrm{H}$ data fit function made by J. Arrington and I. Sick.[53]. Because hydrogen target is $4 \mathrm{~cm}$ extended cell, widths of elastic peaks are larger than those for carbon foil target. 

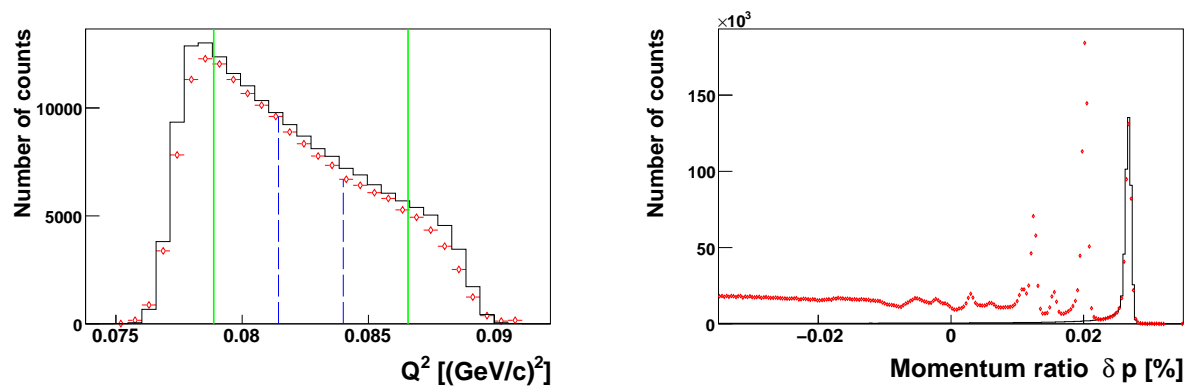

Figure 5.1: Yield of carbon at Kinematics 1.

Left : Yield in $Q^{2}$ spectra. Right : Yield in momentum ratio over the central momentum.
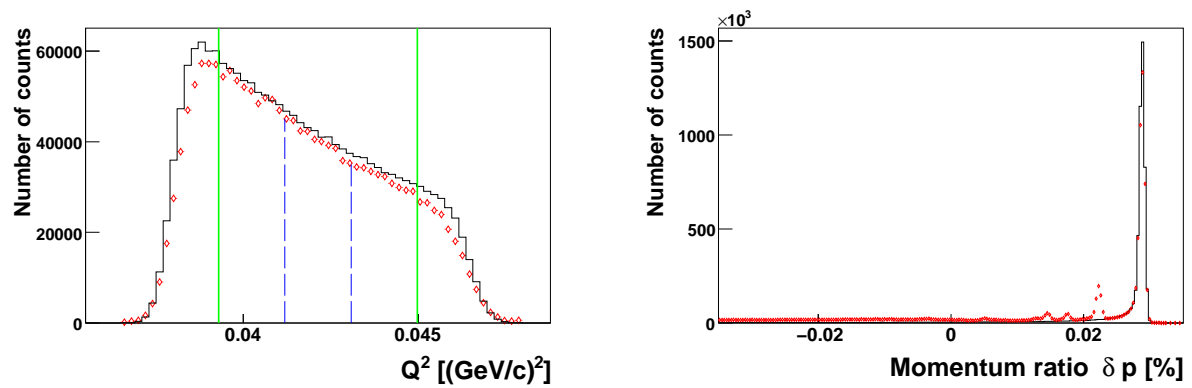

Figure 5.2: Yield of carbon at Kinematics 2.

Left : Yield in $Q^{2}$ spectra. Right : Yield in momentum ratio over the central momentum.
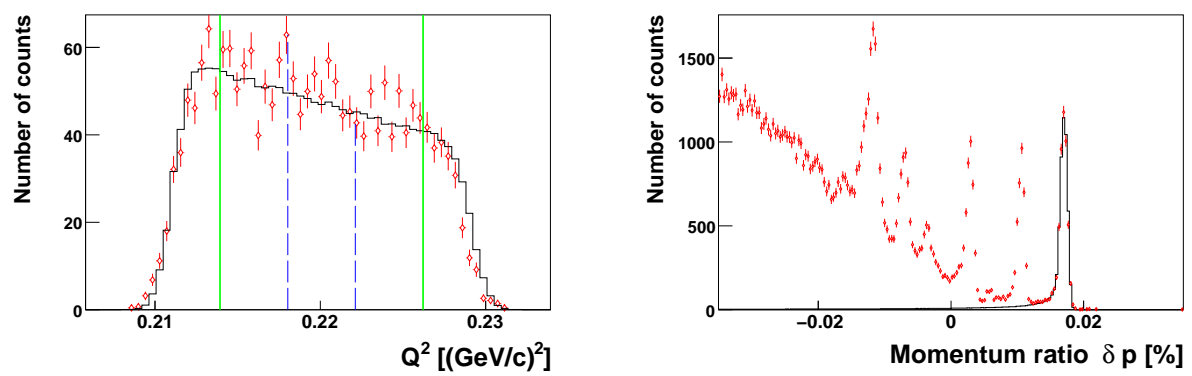

Figure 5.3: Yield of carbon at Kinematics 4.

Left : Yield in $Q^{2}$ spectra. Right : Yield in momentum ratio over the central momentum. 

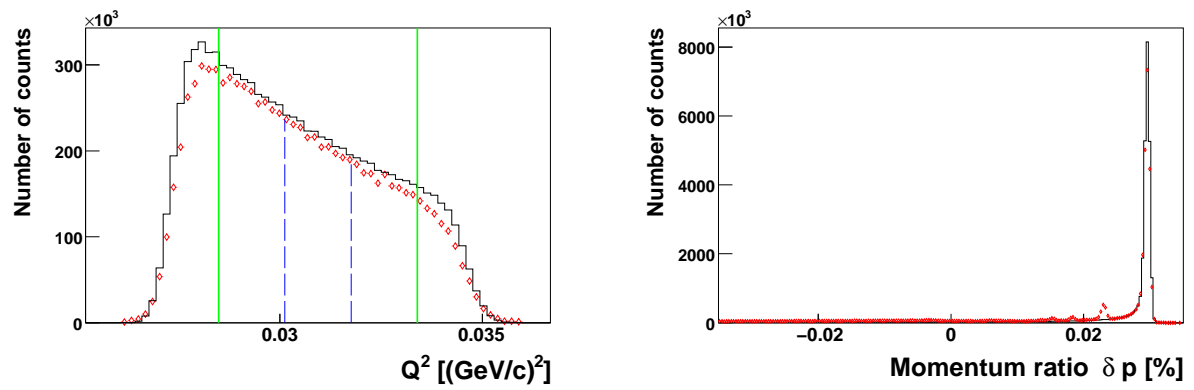

Figure 5.4: Yield of carbon at Kinematics 7.

Left : Yield in $Q^{2}$ spectra. Right : Yield in momentum ratio over the central momentum.
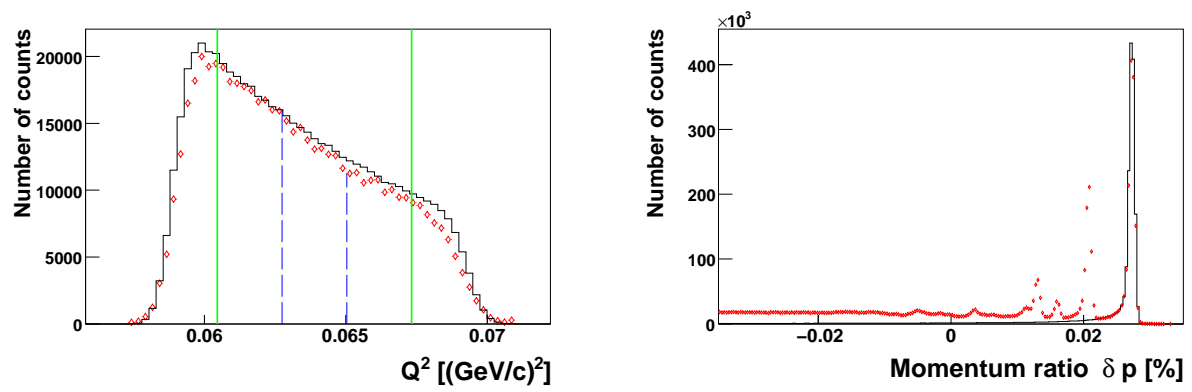

Figure 5.5: Yield of carbon at Kinematics 8.

Left : Yield in $Q^{2}$ spectra. Right : Yield in momentum ratio over the central momentum.
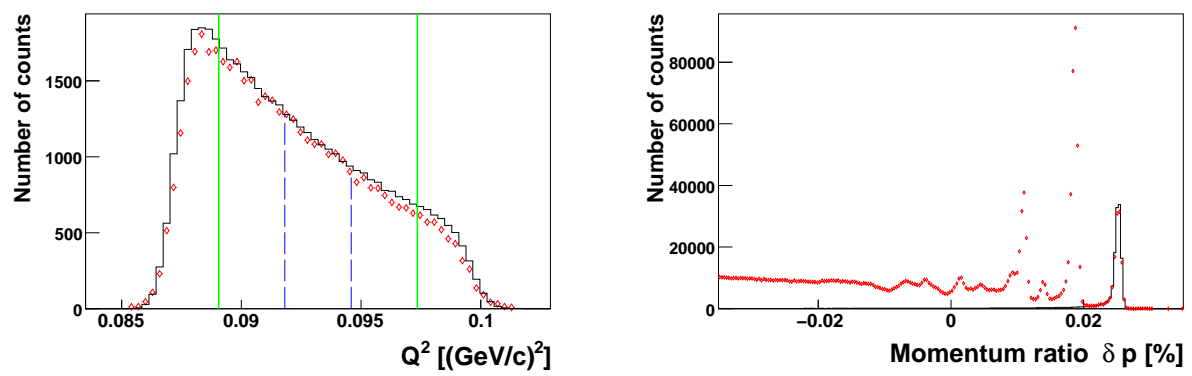

Figure 5.6: Yield of carbon at Kinematics 9.

Left : Yield in $Q^{2}$ spectra. Right : Yield in momentum ratio over the central momentum. 

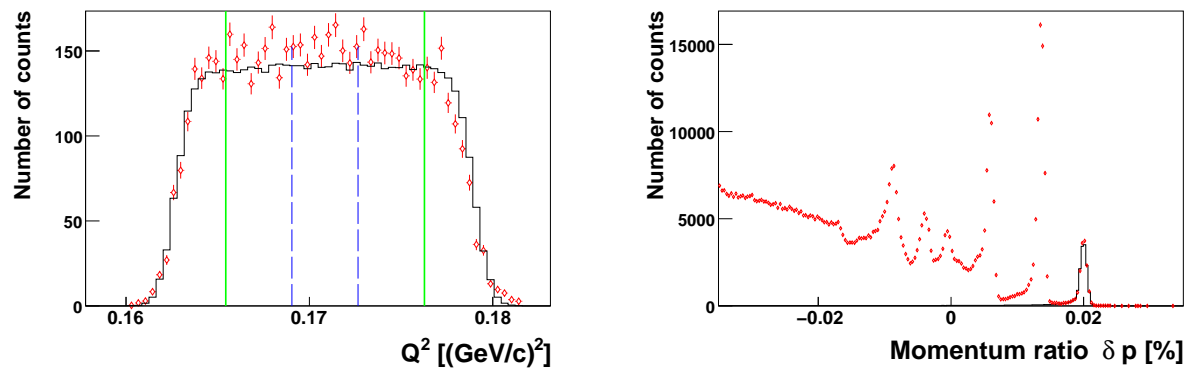

Figure 5.7: Yield of carbon at Kinematics 10.

Left : Yield in $Q^{2}$ spectra. Right : Yield in momentum ratio over the central momentum.
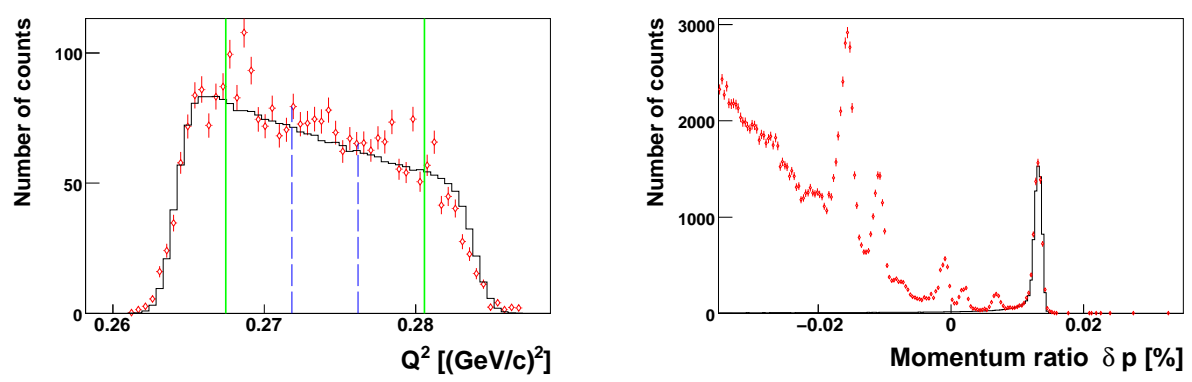

Figure 5.8: Yield of carbon at Kinematics 11.

Left : Yield in $Q^{2}$ spectra. Right : Yield in momentum ratio over the central momentum. 

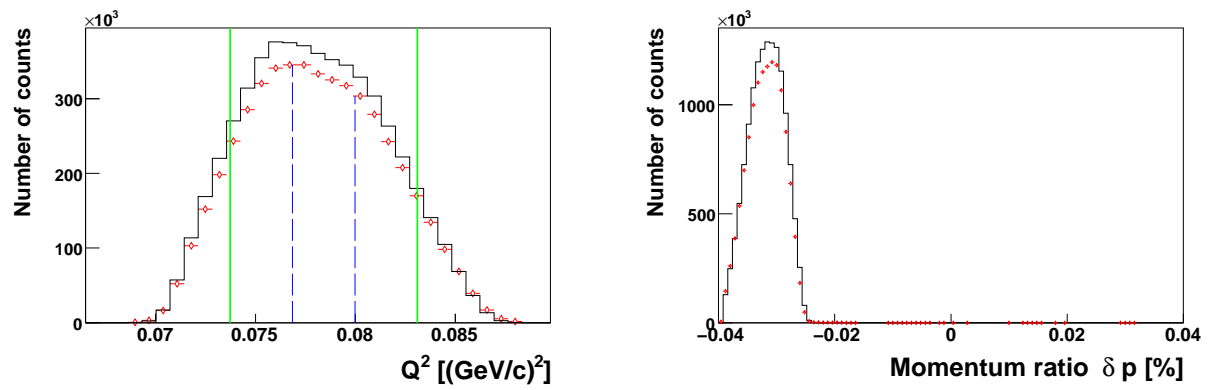

Figure 5.9: Yield of hydrogen at Kinematics 1.

Left : Yield in $Q^{2}$ spectra. Right : Yield in momentum ratio over the central momentum.
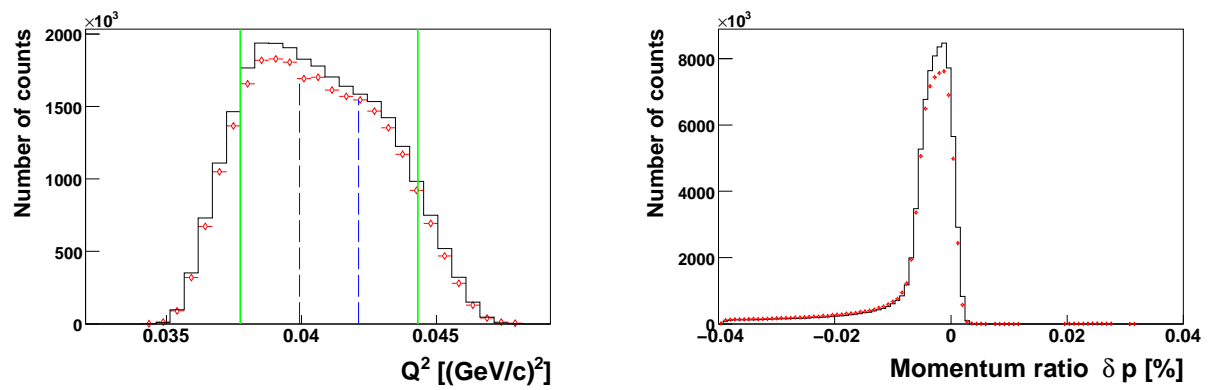

Figure 5.10: Yield of hydrogen at Kinematics 2.

Left : Yield in $Q^{2}$ spectra. Right : Yield in momentum ratio over the central momentum.
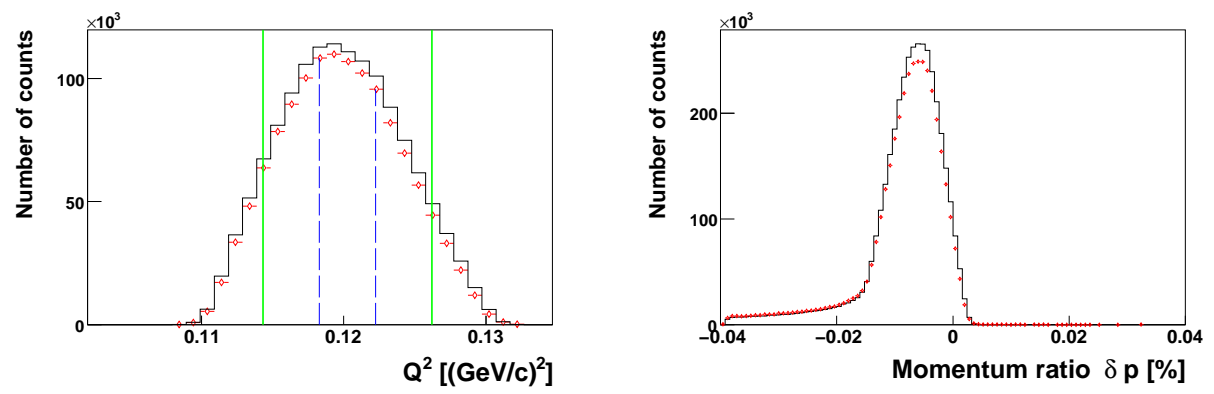

Figure 5.11: Yield of hydrogen at Kinematics 3.

Left : Yield in $Q^{2}$ spectra. Right : Yield in momentum ratio over the central momentum. 

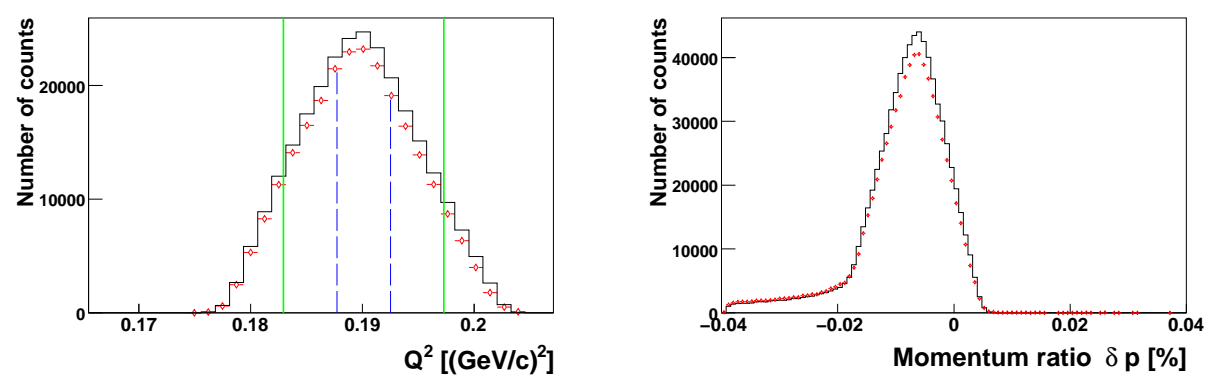

Figure 5.12: Yield of hydrogen at Kinematics 4.

Left : Yield in $Q^{2}$ spectra. Right : Yield in momentum ratio over the central momentum.
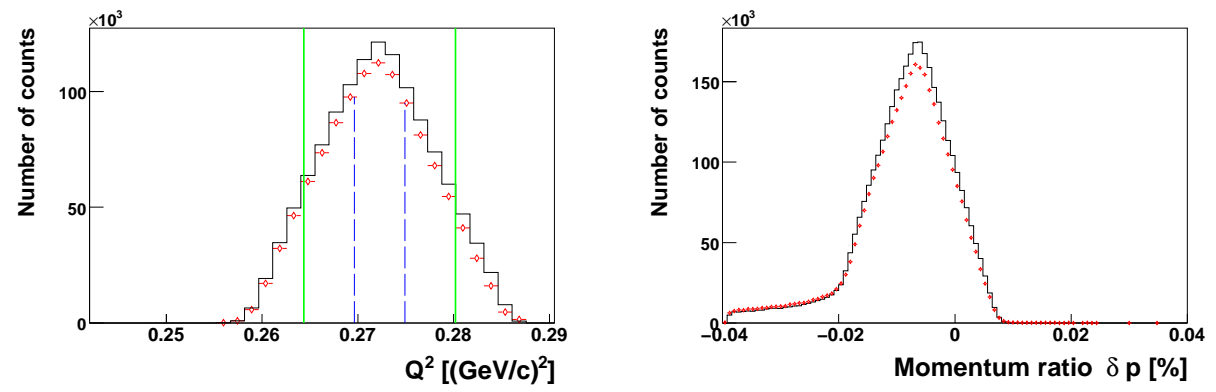

Figure 5.13: Yield of hydrogen at Kinematics 5.

Left : Yield in $Q^{2}$ spectra. Right : Yield in momentum ratio over the central momentum.
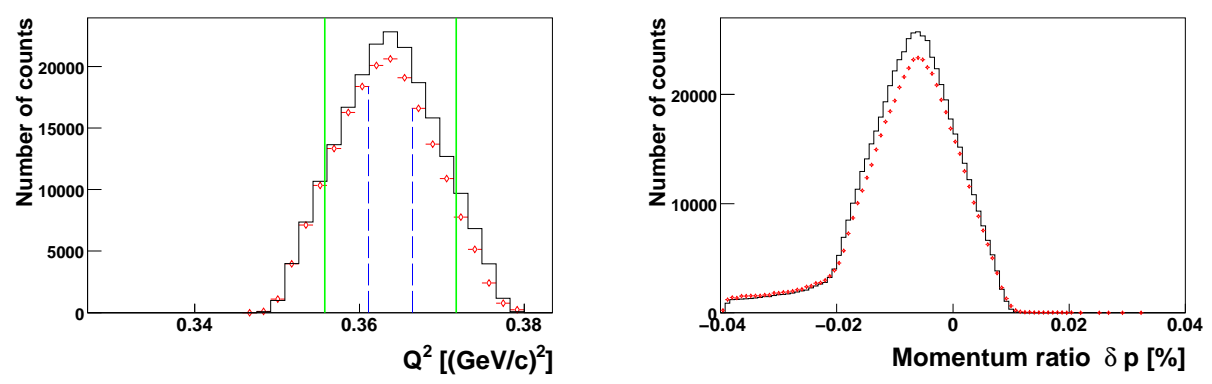

Figure 5.14: Yield of hydrogen at Kinematics 6.

Left : Yield in $Q^{2}$ spectra. Right : Yield in momentum ratio over the central momentum. 

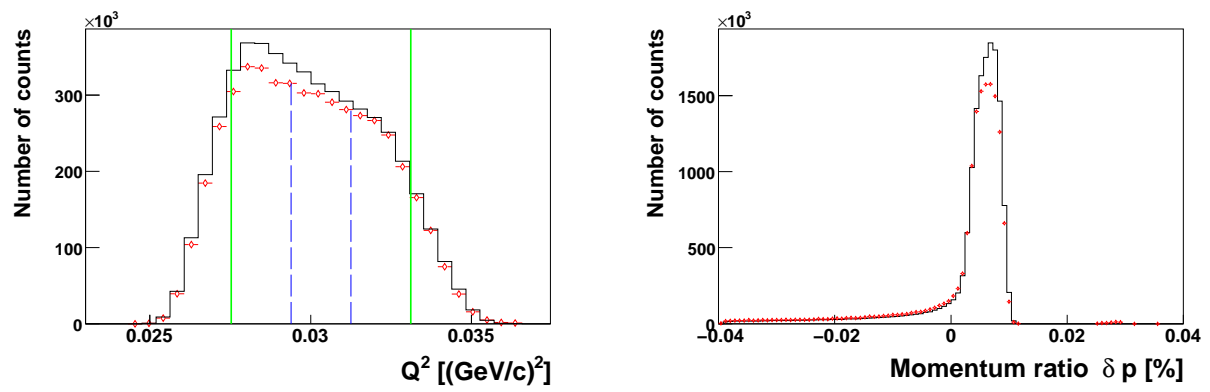

Figure 5.15: Yield of hydrogen at Kinematics 7.

Left : Yield in $Q^{2}$ spectra. Right : Yield in momentum ratio over the central momentum.
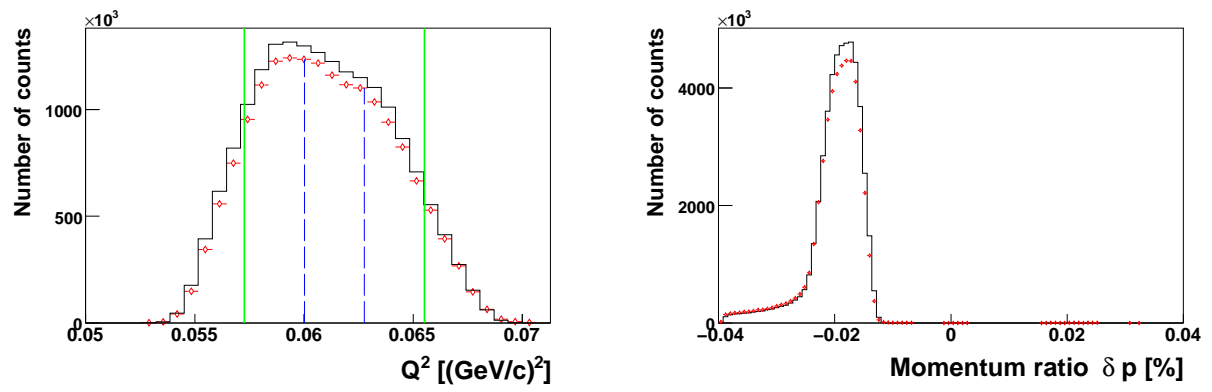

Figure 5.16: Yield of hydrogen at Kinematics 8.

Left : Yield in $Q^{2}$ spectra. Right : Yield in momentum ratio over the central momentum.
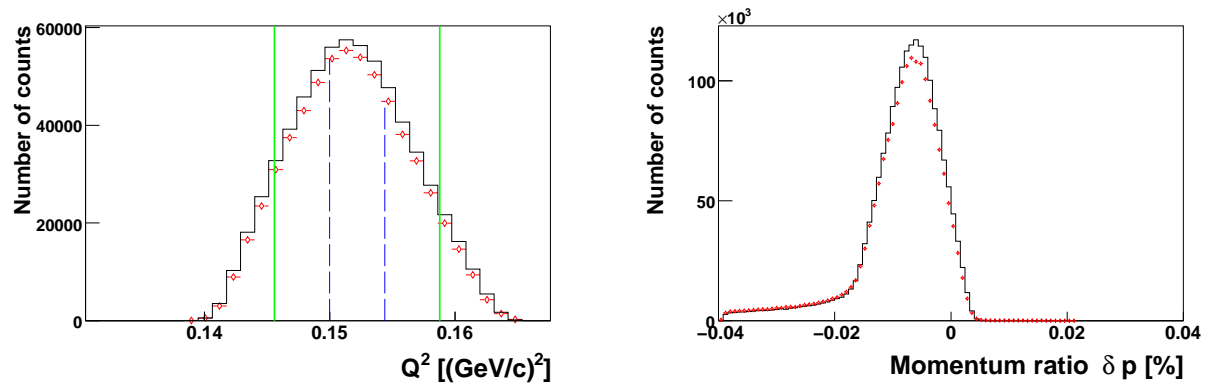

Figure 5.17: Yield of hydrogen at Kinematics 10.

Left : Yield in $Q^{2}$ spectra. Right : Yield in momentum ratio over the central momentum. 

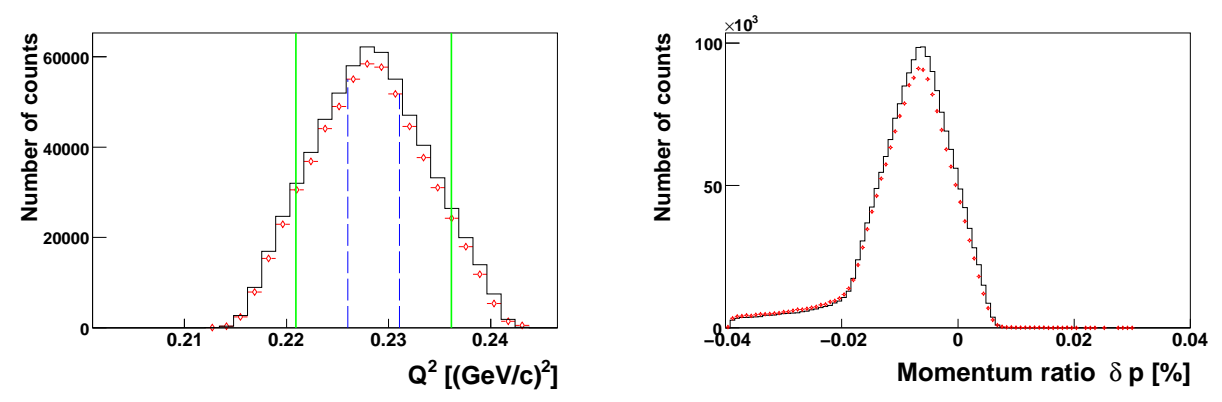

Figure 5.18: Yield of hydrogen at Kinematics 11.

Left : Yield in $Q^{2}$ spectra. Right : Yield in momentum ratio over the central momentum.
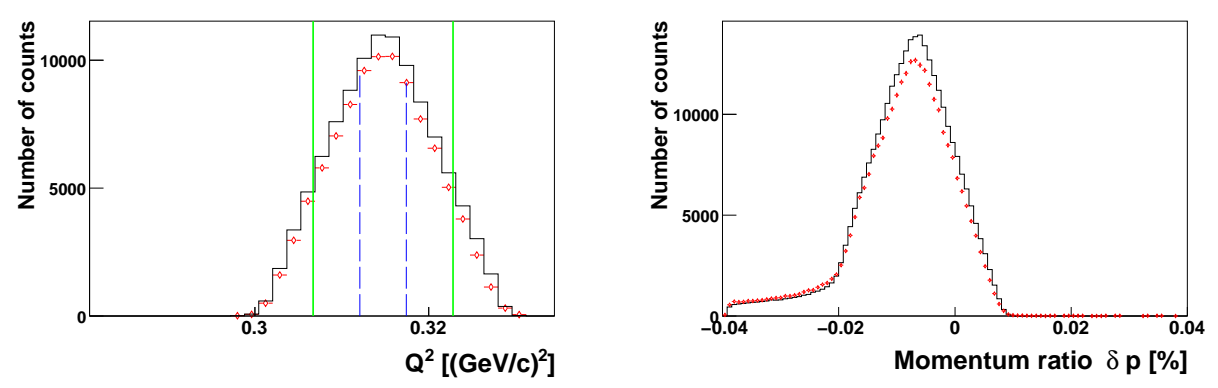

Figure 5.19: Yield of hydrogen at Kinematics 12.

Left : Yield in $Q^{2}$ spectra. Right : Yield in momentum ratio over the central momentum.
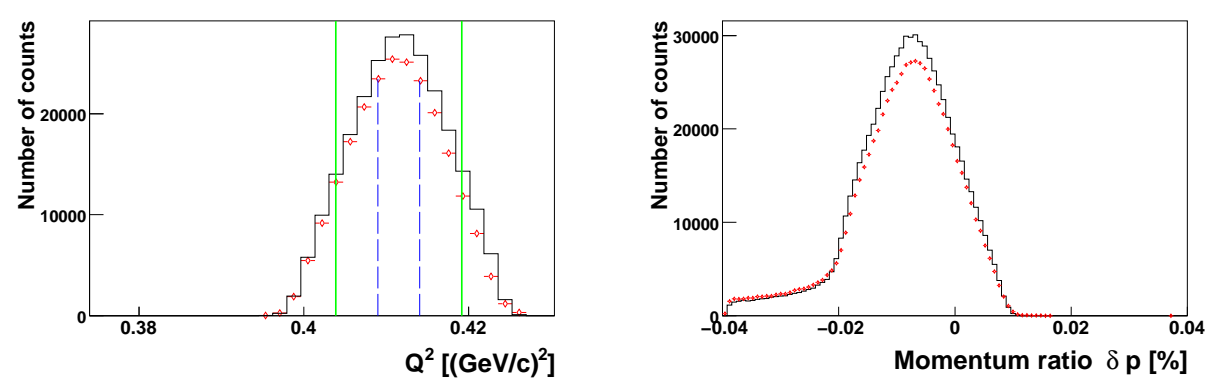

Figure 5.20: Yield of hydrogen at Kinematics 13.

Left : Yield in $Q^{2}$ spectra. Right : Yield in momentum ratio over the central momentum. 


\subsection{Cross sections of ${ }^{12} \mathrm{C}$ and ${ }^{1} \mathrm{H}$}

Values of cross sections and form factor $\left|F\left(Q^{2}\right)\right|^{2}$ are obtained for targets of ${ }^{12} \mathrm{C}$ and ${ }^{1} \mathrm{H}$ and they are shown in this section.

Fig. 5.21 shows obtained values of $\left|F\left(Q^{2}\right)\right|^{2}$ from this experiment for ${ }^{12} \mathrm{C}$ runs. The $x$ axis is $Q^{2}$ in $(\mathrm{GeV} / \mathrm{c})^{2}$ and the $y$ axis is $\left|F\left(Q^{2}\right)\right|^{2} /\left|F\left(Q^{2}\right)\right|_{\text {fit }}^{2}$, the ratio of form factor to the fit function used in MCEEP calculation. The same color indicates that data points are made from the same kinematics. Three $Q^{2}$ data points are made in a kinematics with the same regions of yield distribution in section 5.3 and each point corresponds to the central position of each bin. For three points, the data value in the center region is larger than the values in edge regions. Because the uncertainty of the fit function is $2 \%$ in the region $Q^{2}<0.5(\mathrm{GeV} / \mathrm{c})^{2}$ and $5 \%$ in the region $Q^{2}>0.5(\mathrm{GeV} / \mathrm{c})^{2}$, only five kinematics in the region $Q^{2}<0.5(\mathrm{GeV} / \mathrm{c})^{2}$ are compared. The plot shows that carbon data of this experiment are $96.5 \pm 1.6 \%$ compared to the world data fit function. All data are less than the fit function and there exists a systematic offset of $3.5 \%$.

Fig. 5.22 shows the obtained form factor ratios for ${ }^{1} \mathrm{H}$. The uncertainty of the fit function is $1 \%$ and all kinematics points are drawn here. Hydrogen data of this experiment are $94.3 \pm 1.3 \%$ compared to world data fit. The systematic offset of $5.7 \%$ is observed in ${ }^{1} \mathrm{H}$ data. Hydrogen data show similar trend with carbon data but the offset is larger. It is considered that the difference is generated from the different target type. Whereas the carbon target is thin foil, the hydrogen target is $4 \mathrm{~cm}$ extended cell and the acceptance is complex.

From the two cross section plots, it can be deduced that the systematic offset is generated in some experimental settings. Two conditions should be satisfied for $\left|F\left(Q^{2}\right)\right|^{2}$ of data to agree well with the fit function of world data. First, measurements should be operated correctly. Second, acceptance calculations should be accurate. Other factors such as detector efficiencies and normalization factors could 


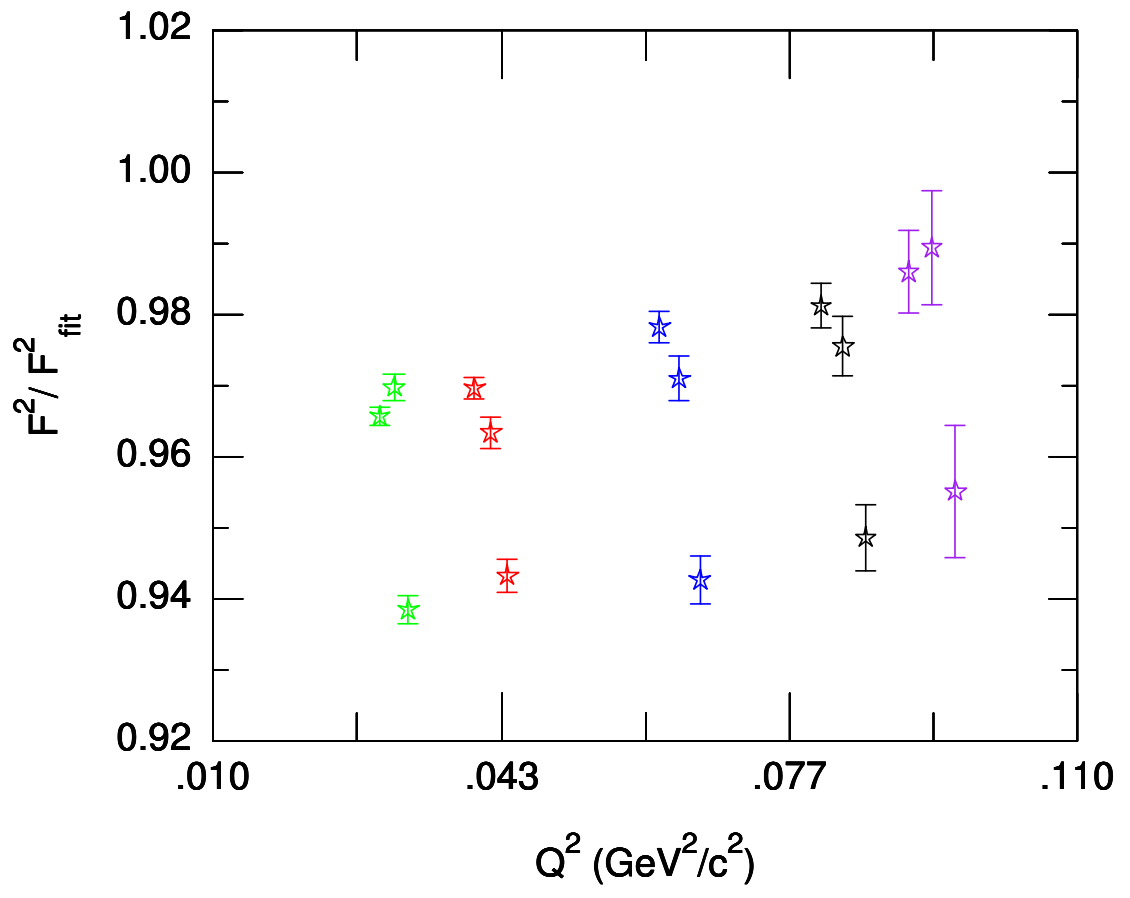

Figure 5.21: Carbon cross section ratio over a world data fit. Data are $96.5 \pm 1.6 \%$ over the fit. 


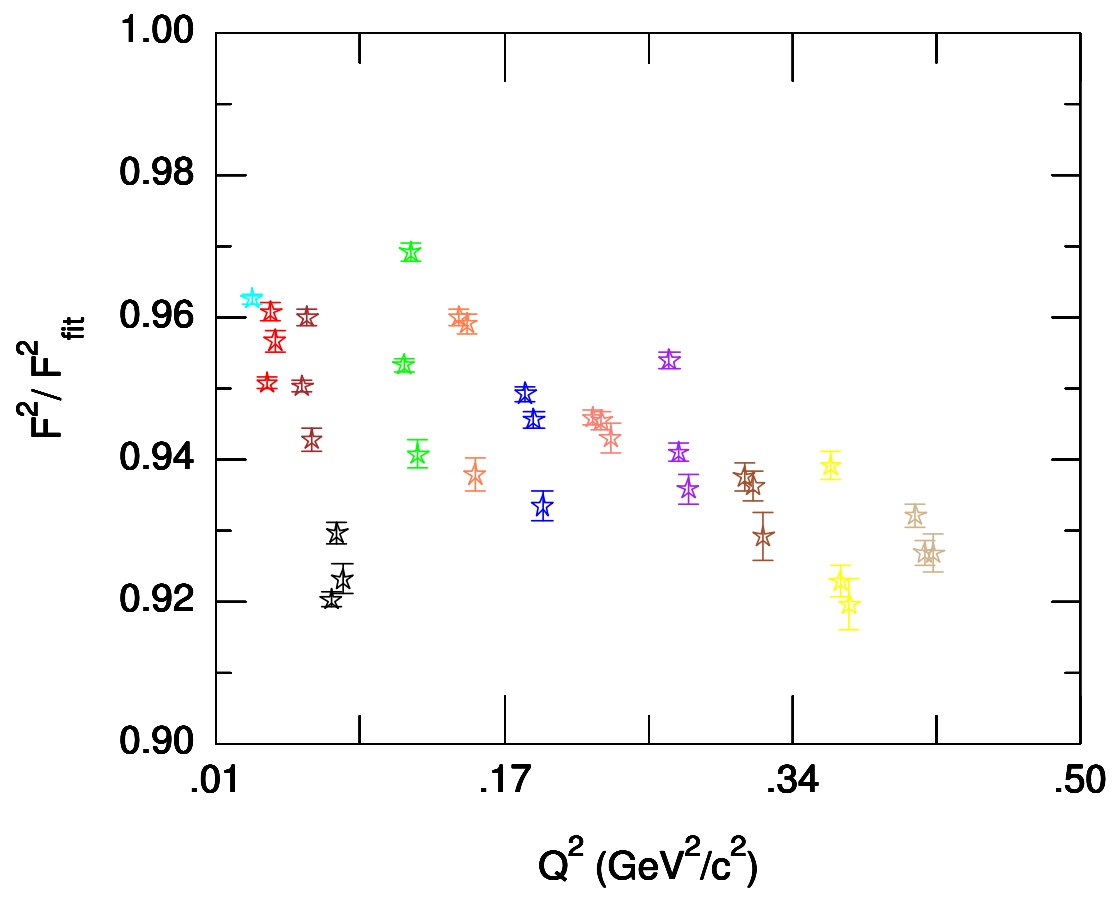

Figure 5.22: Hydrogen cross section ratio over a world data fit. Data are $94.3 \pm 1.3$ $\%$ over the fit. 
be candidates but the possibility is low. In addition, the systematic offset from them can be interpreted as the acceptance decrease or increase either. Two plots show the consistent offset variation over whole kinematic regions and the difference of two offset values is in the range of uncertainty. Hence it is considered that the offsets are generated from the wrong information of acceptance.

It is expected that deuteron data also have offset. However ${ }^{2} \mathrm{D}$ cross section is measured with the ratio to ${ }^{1} \mathrm{H}$ data and deuteron data are free from systematic offset. Further explanations are given in next section.

\subsection{Systematic Offset Correction : Cross section Ratio}

Generally cross section measurements are operated at each data point with a target of interest. On the other hand, cross sections can be measured with two targets. In this case, data of one target(sample target) should be well known from world data. If two targets are measured together and data of sample target are known, data of another target can be extracted from ratios to sample data. Deuteron cross sections of this experiment are measured by the this method. Hydrogen cross sections are

already well known from world data and deuteron cross sections are obtained as ratios to hydrogen data. It is processed by below procedures.

1. Hydrogen cross sections are obtained in $\mathrm{N}$ points.

2. Deuteron cross sections are obtained in $\mathrm{N}$ points with the same kinematics of hydrogen.

3. Hydrogen data are compared with the world data fit like Fig. 5.22. And then offset ratios $E(Q)$ are obtained at every point.

4. Deuteron cross sections are divided by the ratios $E(Q)$ at every point. 
It is expected that the offset value and variation pattern would be similar with ones from hydrogen data because no kinematic difference exists between the two targets. If ratios between the two targets are obtained, offsets between them can be canceled. Hence deuteron cross sections are not affected by the systematic offset and accurate values are obtained. 



\section{Chapter 6}

\section{Results}

After all works in previous chapters, deuteron cross sections are obtained. As seen in Eq. 1.8, the deuteron cross section can be divided into the non-structure cross section and form factor term of $A(Q)$ and $B(Q)$. A fit function is made from world data of $B(Q)$ across the region $Q<1 \mathrm{GeV} / \mathrm{c}$ and then it is subtracted from cross sections to extract values of $A(Q)$ data. In this chapter, obtained values of $A(Q)$ and cross section are given and they are compared with world data and theoretical calculations.

\subsection{Cross section of Deuteron}

Elastic cross sections of three targets of ${ }^{12} \mathrm{C},{ }^{1} \mathrm{H}$ and ${ }^{2} \mathrm{D}$ are measured during this experiment. Cross section measurements of ${ }^{12} \mathrm{C}$ and ${ }^{1} \mathrm{H}$ show approximately $5 \%$ systematic offset. The offset is cancelled by the method of cross section ratios to hydrogen data and the effective offset is approximately $0.8 \%$. It is included to the systematic error as acceptance uncertainty. Cross sections of ${ }^{2} \mathrm{D}$ are obtained at all kinematics and they are drawn over $Q^{2}$ in Fig. 6.1 - 6.11. Final values are in Table 6.1. Two red vertical lines define the edges of data selection in acceptance region and green lines separate regions of data points. Data points are made at 
the center of each region between two vertical lines. The $x$ axis is $Q^{2}$ in $(\mathrm{GeV} / \mathrm{c})^{2}$ and the $y$ axis is the cross section in $\mathrm{mb} / \mathrm{sr}$ Three data points are made at each kinematics except a kinematics with the highest trigger rate. In the kinematics, the trigger rate was too high $(\sim 200 \mathrm{kHz})$ so detectors might not work correctly. And variations of cross section values in the kinematics are bigger than those from other kinematics. Hence only one data point(average over three region) is made at the kinematics. The momentum setting of Kin. 9 was out of the acceptance region during this experiment and the result is omitted.

\section{2 $\mathrm{B}(\mathrm{Q})$ Fit Function}

If the Rosenbluth separation is used, values of $A(Q)$ and $B(Q)$ can be obtained directly from deuteron cross sections. However this analysis used data with one beam energy of $680 \mathrm{MeV}$ and another data set with different beam energy are required for the Rosenbluth separation. This experiment is focused on obtaining accurate $A(Q)$ values instead. And kinematic settings are chosen to suppress the $B(Q)$ term contribution as small as possible. The contribution of $B(Q)$ term is less than $1 \%$ across the problematic region of $0.2<Q<0.4 \mathrm{GeV} / \mathrm{c}$.

A new fit function is made from world data of $B(Q)$ at the region $Q<1 \mathrm{GeV} / \mathrm{c}$ and then the function values are subtracted from deuteron cross sections. There exists an old fit function of Eq. 6.1 over the whole data range [4]. Fig. 6.13 compares world data of $\mathrm{B}(\mathrm{Q})$ with the old function across the region $Q<1 \mathrm{GeV} /$ c. The $x$ axis is $Q$ in $\mathrm{GeV} / \mathrm{c}$ and the $y$ axis is the ratio of $B(Q)$ world data to the old fit function. 


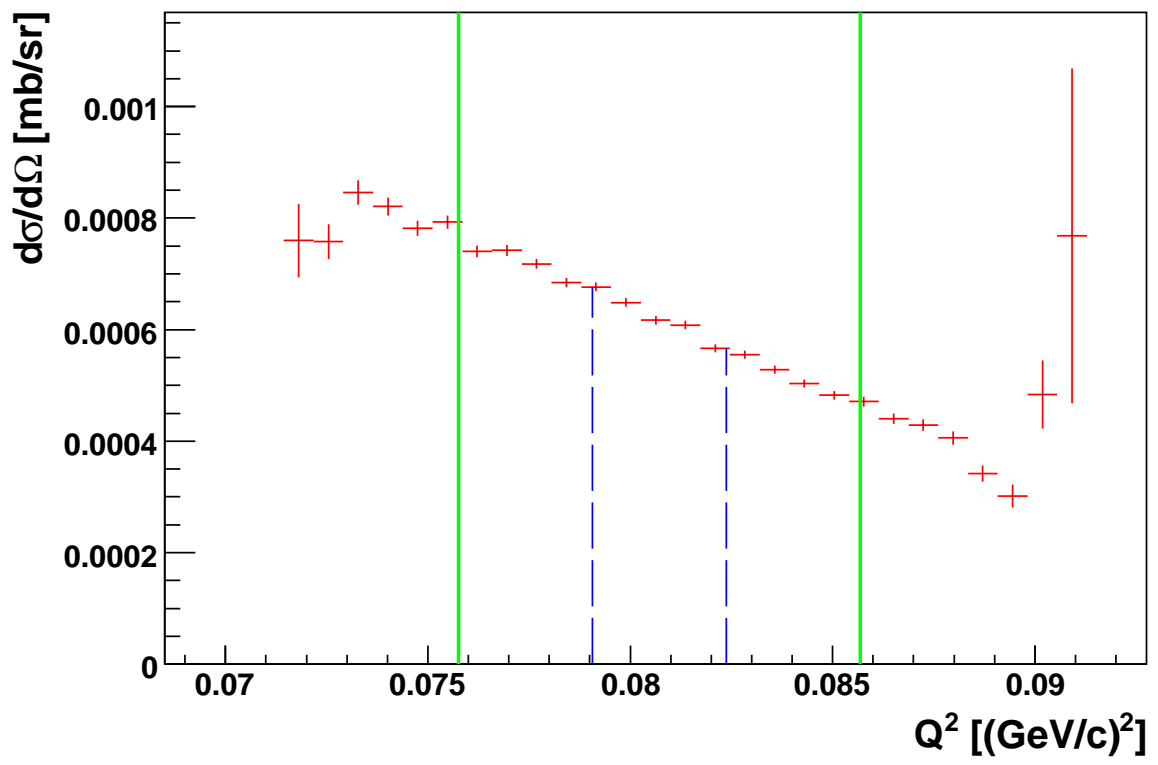

Figure 6.1: Cross sections from Kinematics 1.

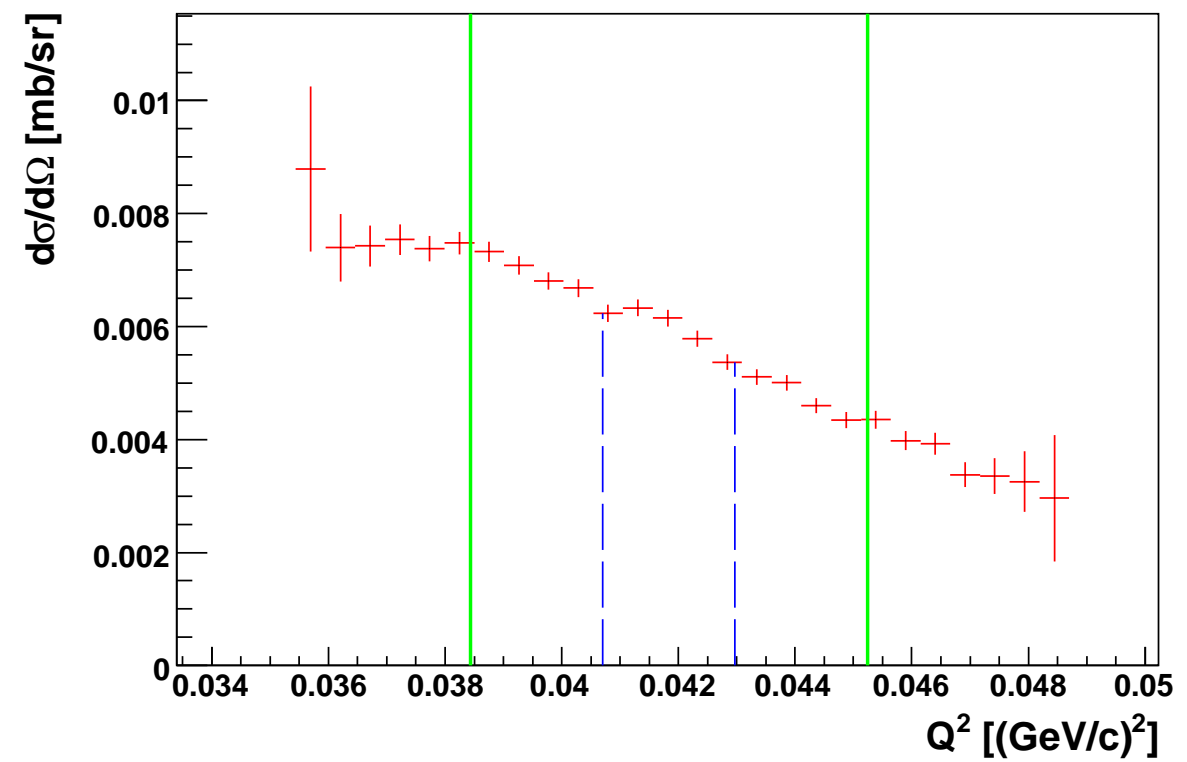

Figure 6.2: Cross sections from Kinematics 2. 


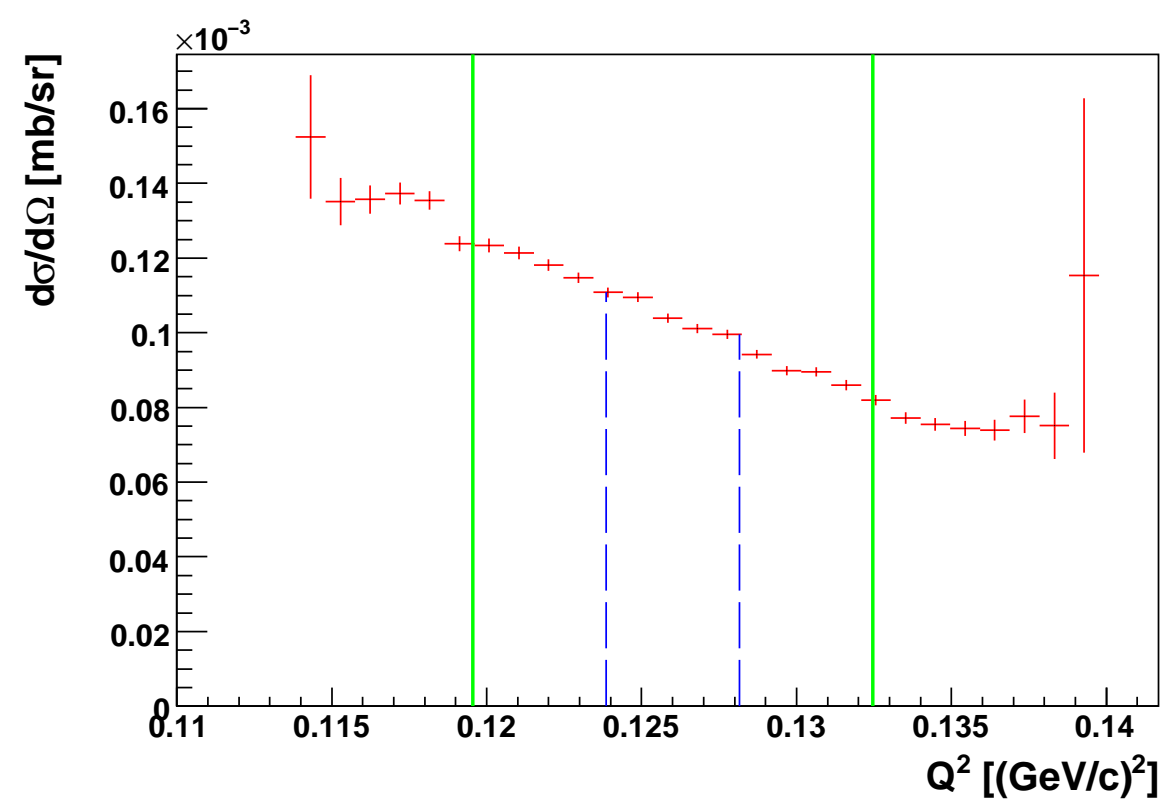

Figure 6.3: Cross sections from Kinematics 3.

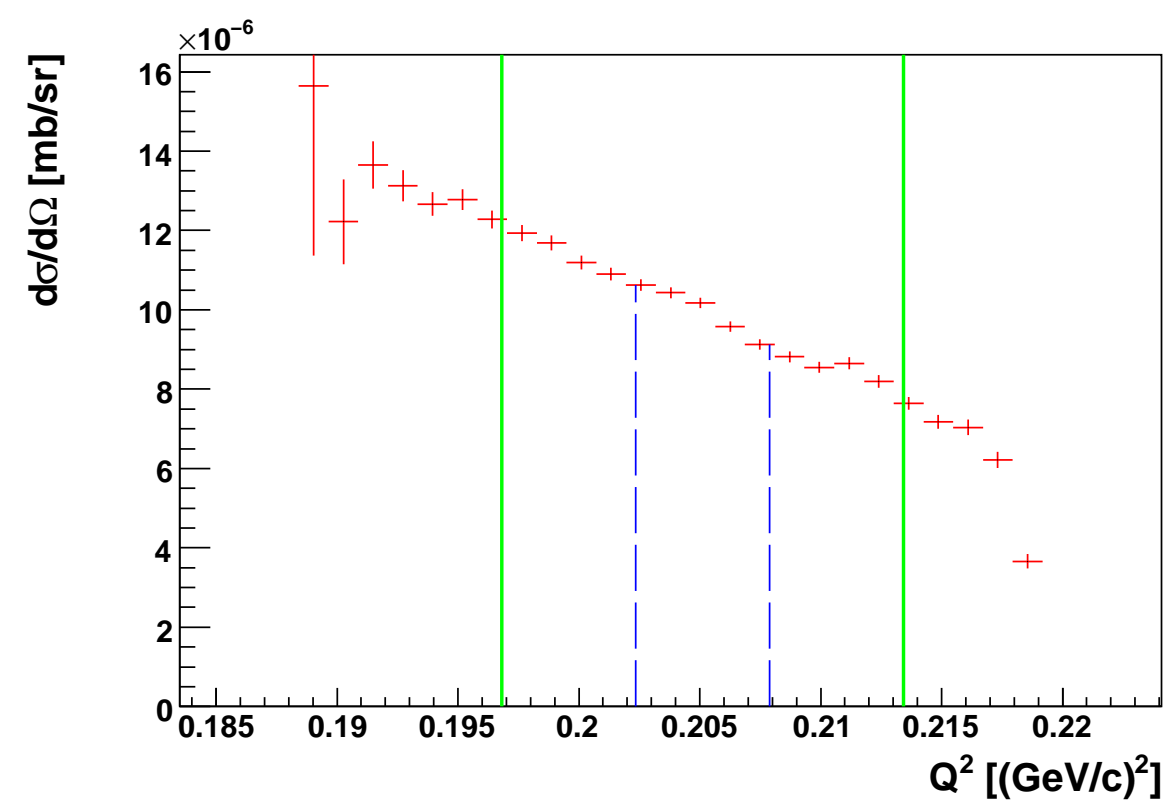

Figure 6.4: Cross sections from Kinematics 4. 


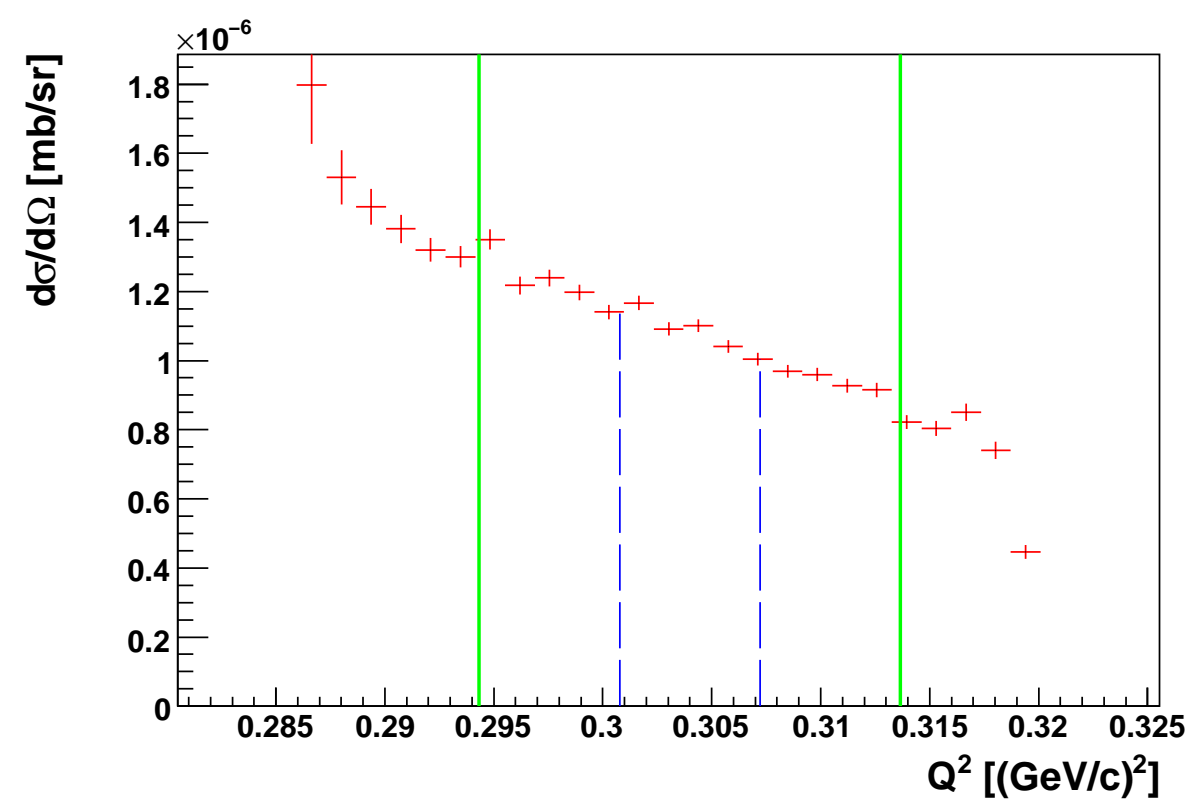

Figure 6.5: Cross sections from Kinematics 5.

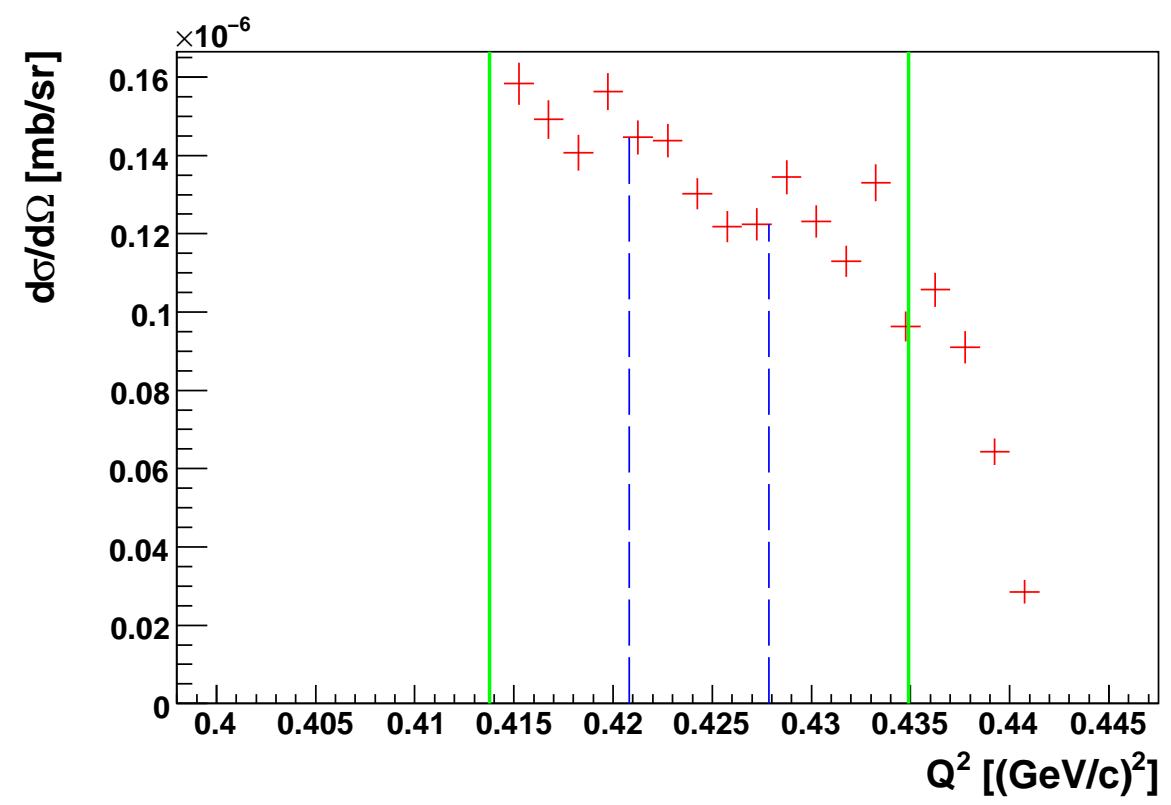

Figure 6.6: Cross sections from Kinematics 6. 


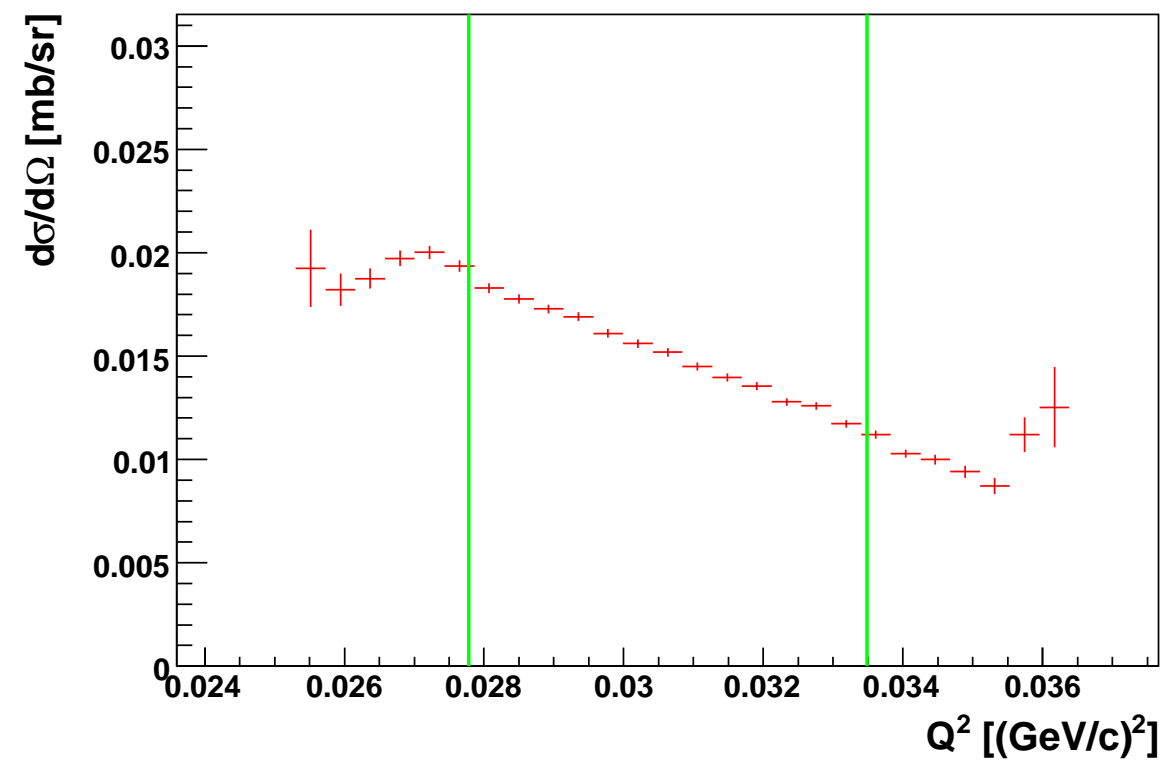

Figure 6.7: Cross section from Kinematics 7.

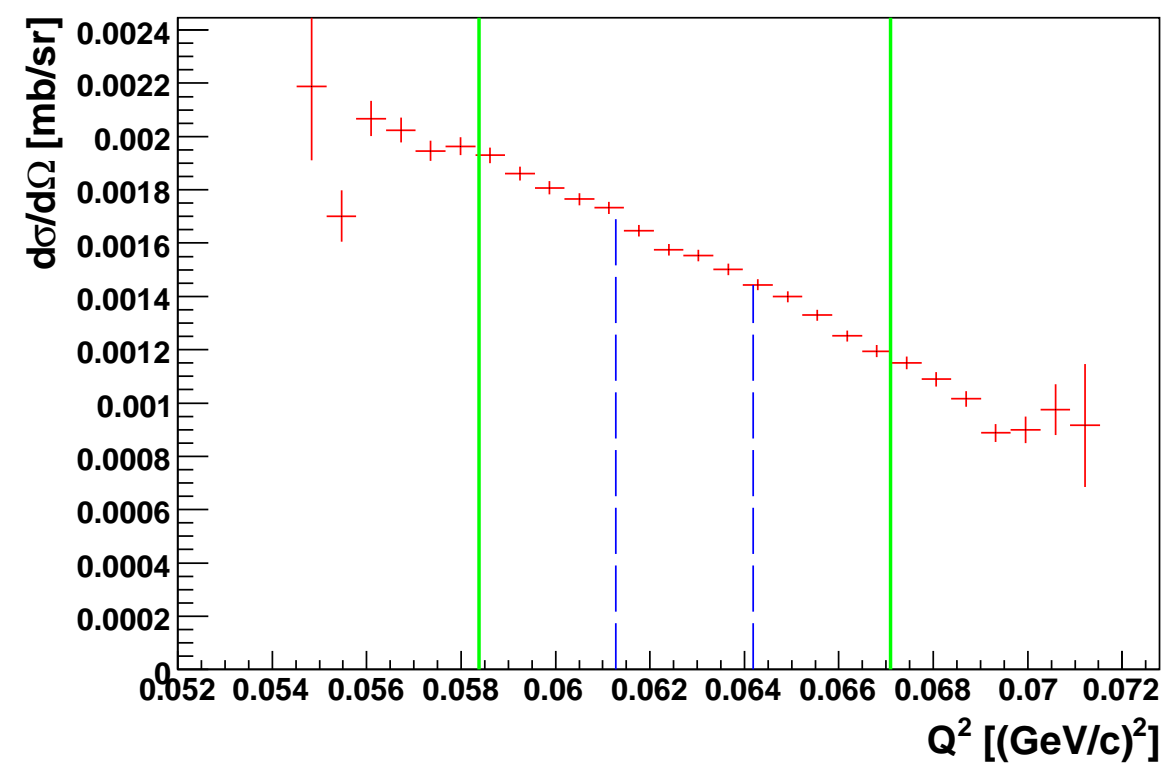

Figure 6.8: Cross sections from Kinematics 8. 


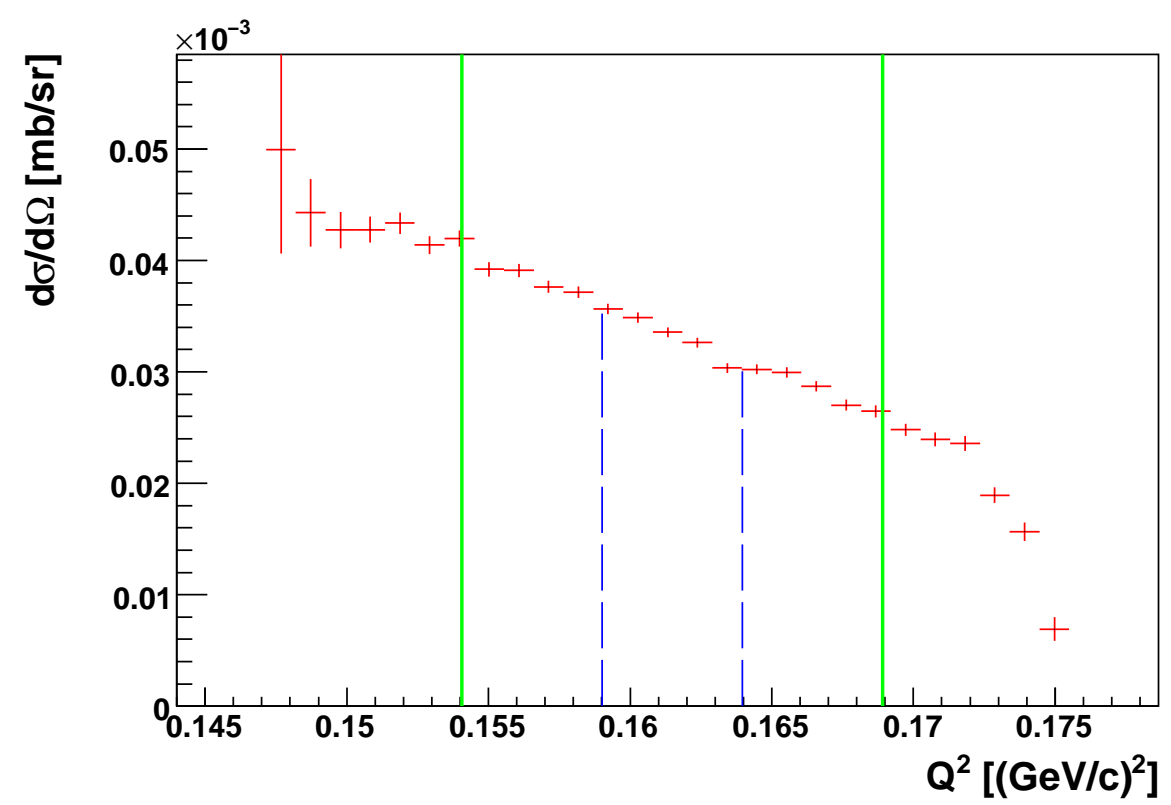

Figure 6.9: Cross sections from Kinematics 10.

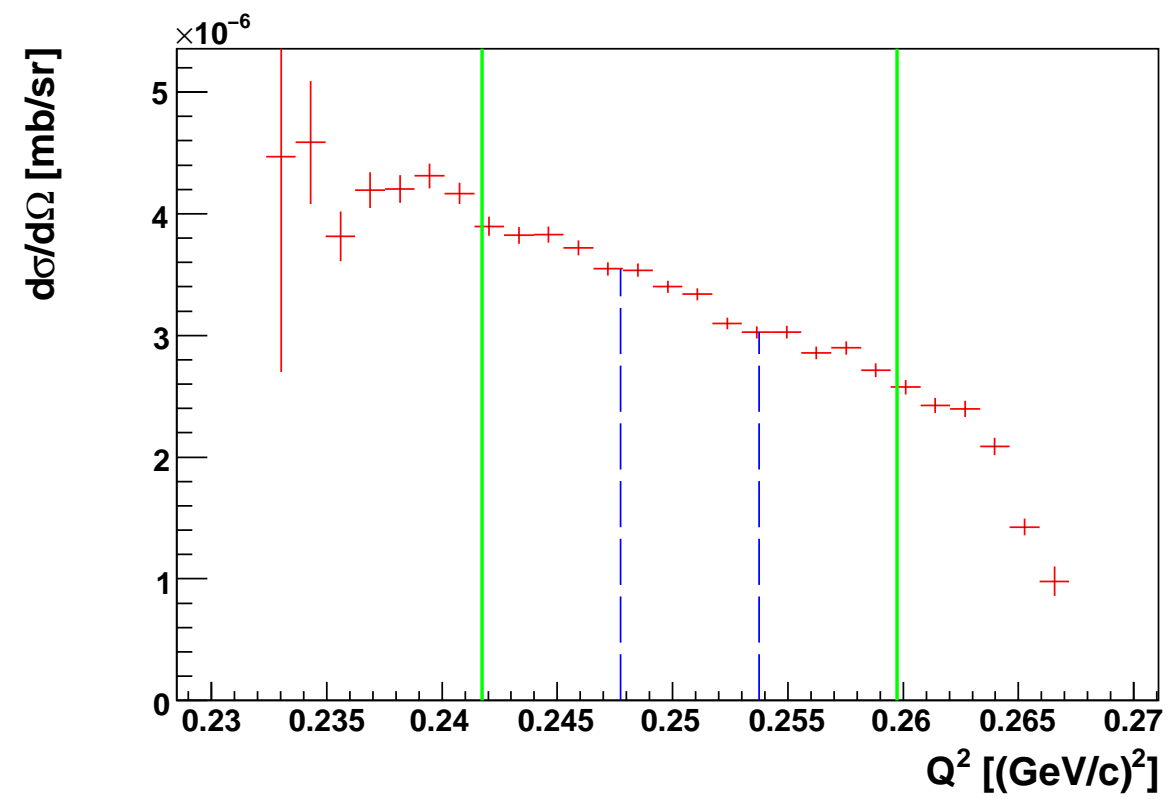

Figure 6.10: Cross sections from Kinematics 11. 


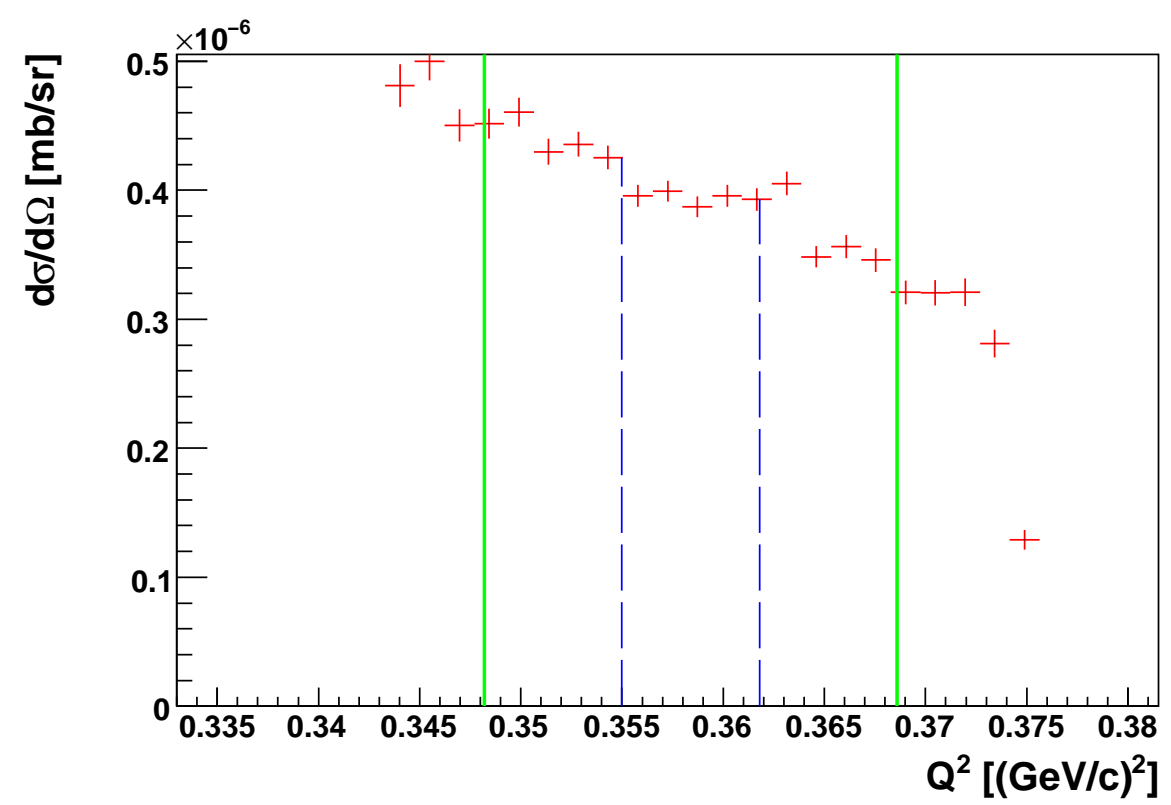

Figure 6.11: Cross sections from Kinematics 12.

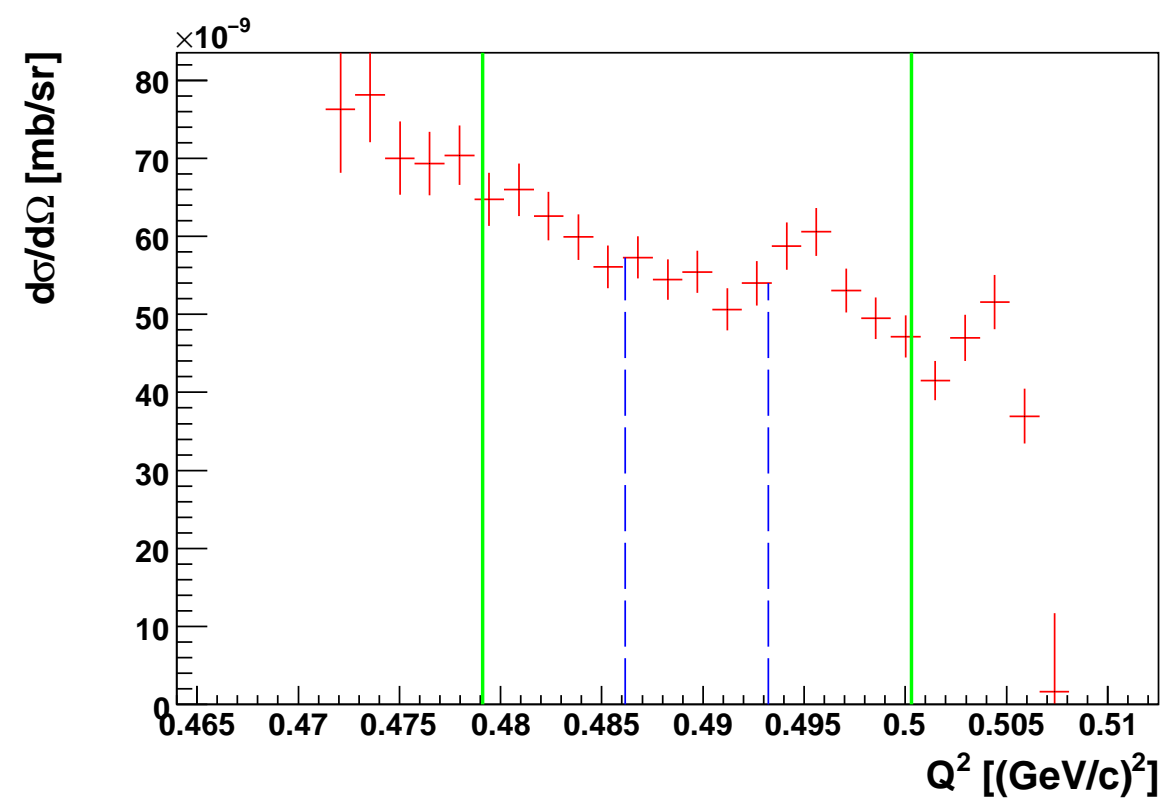

Figure 6.12: Cross sections from Kinematics 13. 


$$
\begin{aligned}
f(Q) & =\frac{1}{\left(1+Q^{2} / C\right)\left(1+Q^{2} / 0.71\right)^{2}} \\
G_{C} & =e^{\left(-Q^{2} / 3.5\right) f(Q)} \\
G_{Q} & =\frac{25.8298}{1.01}\left(e^{-Q^{2}}+0.01 e^{-0.01 Q^{2}}\right) f(Q) \\
G_{M} & =1.7487 \times e^{-Q^{2} / 2.5} f(Q) \\
B(Q) & =\frac{4}{3} \eta(1+\eta) \cdot G_{M}^{2},
\end{aligned}
$$

where, $\eta=Q^{2} /\left(4 M^{2}\right)$ and $C=0.936 \times 0.0022246 / 0.197 / 0.197$. There exist at most $50 \%$ difference between data and the old function. Therefore a new fit function of better agreement can be obtained with slight modification. An additional $2^{\text {nd }}$ order

polynomial is added to the function and a new fit function is found with reduced $\chi^{2} \approx 1$. The new fit function is given as

$$
B(Q)_{\text {new }}=\frac{4}{3} \cdot \eta \cdot(1+\eta) \cdot G_{m}^{2} \cdot\left(0.8097+3.067 \sqrt{Q^{2}}-3.398 Q^{2}\right) .
$$

The black line in 6.13 is the new fit function and the ratio of $B(Q)$ to the new fit function is drawn in Fig. 6.14.

\section{3 $\mathrm{A}(\mathrm{Q})$ Data}

Values of deuteron $A(Q)$ structure function are extracted from the new $B(Q)$ fit function and cross sections by Eq. 6.3 .

$$
A(Q)=\frac{\mathrm{d} \sigma}{\mathrm{d} \Omega} /\left.\frac{\mathrm{d} \sigma}{\mathrm{d} \Omega}\right|_{\mathrm{NS}}-B(Q) \tan ^{2} \frac{\theta}{2}
$$

Extracted values of $A(Q)$ data are written in Table 6.1 together with cross sections and errors. The beam energy is $685 \mathrm{MeV}$ at all data points. 


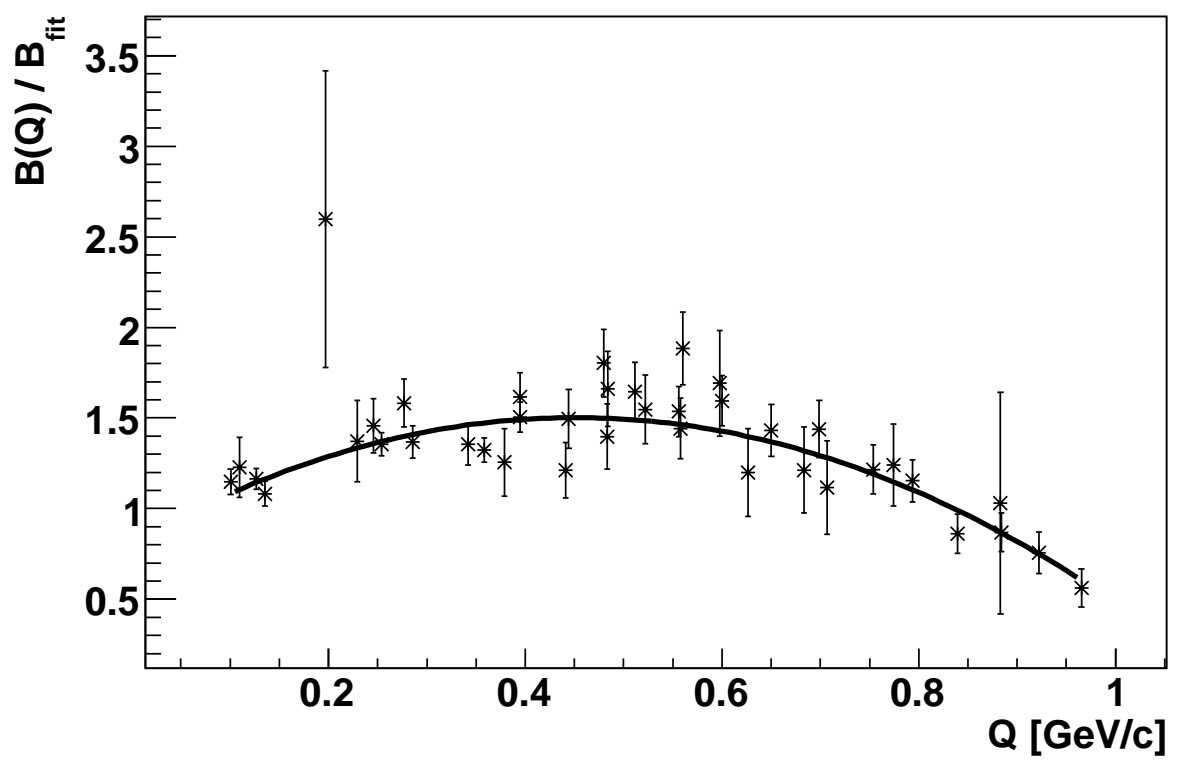

Figure 6.13: $B(Q) / \mathrm{B}_{\text {fit }}$ vs. Q.

A fit(black line) of 2nd order polynomial is newly applied to update the old fit function $\mathrm{B}_{\text {fit }}$.

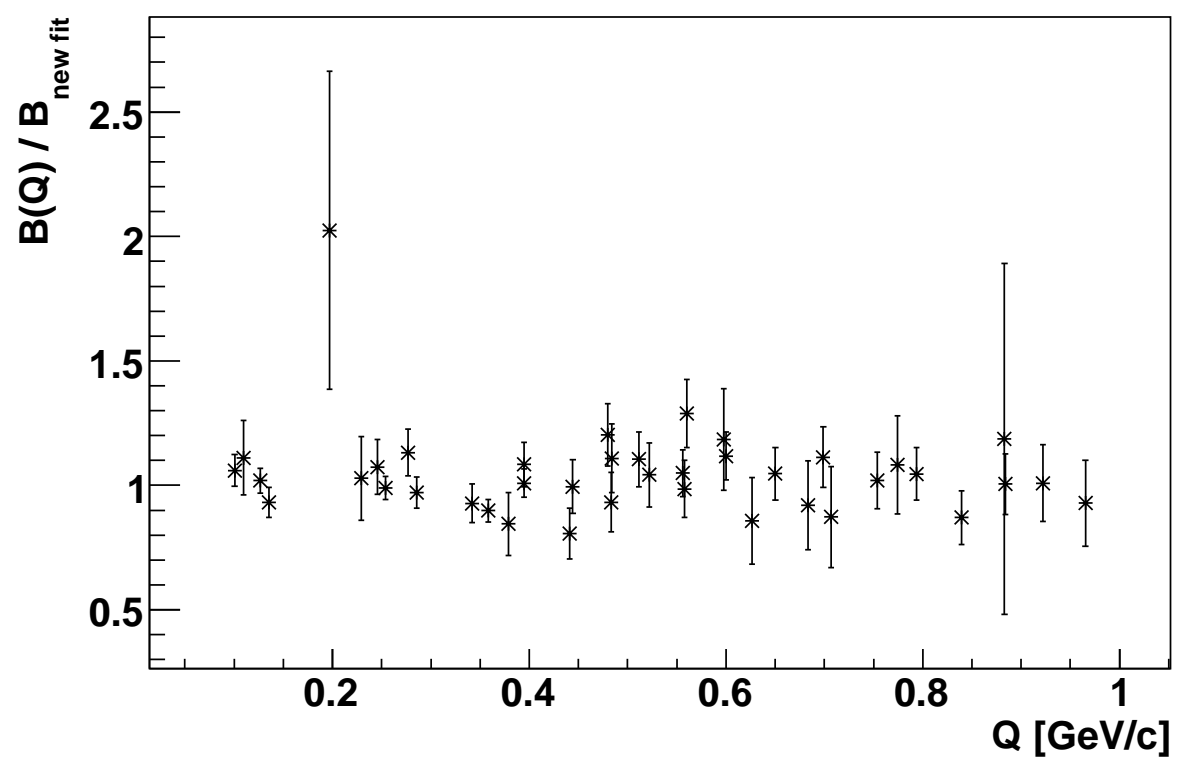

Figure 6.14: $B(Q) /($ New fit function) vs. Q.

The $y$ axis is the ratio of $B(Q)$ data to the modified function. 
Table 6.1: Values of $A(Q)$ and deuteron cross sections.

\begin{tabular}{|c|c|c|c|c|c|c|}
\hline $\begin{array}{c}Q \\
{[\mathrm{GeV} / \mathrm{c}]}\end{array}$ & $\begin{array}{c}Q^{2} \\
{\left[\mathrm{fm}^{-2}\right]}\end{array}$ & $A(Q)$ & $A(Q) / A_{\text {fit }}$ & $\begin{array}{c}\text { Cross section } \\
{[\mathrm{mb} / \text { srad }]}\end{array}$ & $\begin{array}{c}\text { Statistical } \\
\text { uncertainty } \\
{[\%]}\end{array}$ & $\begin{array}{c}\text { Systematic } \\
\text { uncertainty } \\
{[\%]}\end{array}$ \\
\hline 0.175 & 0.785 & $3.79 \mathrm{e}-1$ & 1.12 & $1.50 \mathrm{e}-04$ & 0.1 & 1.2 \\
0.199 & 1.015 & $2.99 \mathrm{e}-1$ & 1.13 & $6.96 \mathrm{e}-05$ & 0.2 & 1.4 \\
0.204 & 1.073 & $2.84 \mathrm{e}-1$ & 1.14 & $5.90 \mathrm{e}-05$ & 0.3 & 1.4 \\
0.210 & 1.132 & $2.65 \mathrm{e}-1$ & 1.13 & $4.92 \mathrm{e}-05$ & 0.4 & 1.4 \\
0.245 & 1.535 & $1.83 \mathrm{e}-1$ & 1.15 & $1.80 \mathrm{e}-05$ & 0.2 & 1.3 \\
0.250 & 1.609 & $1.74 \mathrm{e}-1$ & 1.16 & $1.55 \mathrm{e}-05$ & 0.3 & 1.3 \\
0.256 & 1.685 & $1.61 \mathrm{e}-1$ & 1.15 & $1.30 \mathrm{e}-05$ & 0.4 & 1.3 \\
0.278 & 1.987 & $1.28 \mathrm{e}-1$ & 1.17 & $7.28 \mathrm{e}-06$ & 0.2 & 1.3 \\
0.284 & 2.071 & $1.21 \mathrm{e}-1$ & 1.19 & $6.32 \mathrm{e}-06$ & 0.3 & 1.3 \\
0.290 & 2.157 & $1.12 \mathrm{e}-1$ & 1.18 & $5.36 \mathrm{e}-06$ & 0.4 & 1.3 \\
0.349 & 3.124 & $5.47 \mathrm{e}-2$ & 1.12 & $1.16 \mathrm{e}-06$ & 0.2 & 1.5 \\
0.355 & 3.234 & $5.14 \mathrm{e}-2$ & 1.12 & $1.01 \mathrm{e}-06$ & 0.2 & 1.5 \\
0.361 & 3.345 & $4.88 \mathrm{e}-2$ & 1.14 & $8.92 \mathrm{e}-07$ & 0.4 & 1.5 \\
0.396 & 4.019 & $3.13 \mathrm{e}-2$ & 1.08 & $3.78 \mathrm{e}-07$ & 0.4 & 1.3 \\
0.402 & 4.145 & $2.93 \mathrm{e}-2$ & 1.08 & $3.29 \mathrm{e}-07$ & 0.4 & 1.3 \\
0.408 & 4.273 & $2.71 \mathrm{e}-2$ & 1.07 & $2.84 \mathrm{e}-07$ & 0.7 & 1.3 \\
0.447 & 5.124 & $1.62 \mathrm{e}-2$ & 0.97 & $1.11 \mathrm{e}-07$ & 0.4 & 1.3 \\
0.453 & 5.266 & $1.53 \mathrm{e}-2$ & 0.99 & $9.87 \mathrm{e}-08$ & 0.4 & 1.3 \\
0.459 & 5.409 & $1.43 \mathrm{e}-2$ & 0.98 & $8.60 \mathrm{e}-08$ & 0.8 & 1.3 \\
0.495 & 6.284 & $8.88 \mathrm{e}-3$ & 0.89 & $3.72 \mathrm{e}-08$ & 0.5 & 1.5 \\
0.501 & 6.437 & $8.28 \mathrm{e}-3$ & 0.88 & $3.27 \mathrm{e}-08$ & 0.5 & 1.5 \\
0.507 & 6.592 & $7.66 \mathrm{e}-3$ & 0.87 & $2.85 \mathrm{e}-08$ & 0.6 & 1.5 \\
0.545 & 7.640 & $4.62 \mathrm{e}-3$ & 0.78 & $1.19 \mathrm{e}-08$ & 0.5 & 1.3 \\
0.551 & 7.805 & $4.37 \mathrm{e}-3$ & 0.79 & $1.06 \mathrm{e}-08$ & 0.6 & 1.3 \\
0.557 & 7.971 & $4.11 \mathrm{e}-3$ & 0.78 & $9.45 \mathrm{e}-09$ & 1.0 & 1.3 \\
0.593 & 9.029 & $2.63 \mathrm{e}-3$ & 0.72 & $4.35 \mathrm{e}-09$ & 0.9 & 2.8 \\
0.599 & 9.202 & $2.49 \mathrm{e}-3$ & 0.72 & $3.91 \mathrm{e}-09$ & 0.9 & 2.8 \\
0.604 & 9.378 & $2.46 \mathrm{e}-3$ & 0.75 & $3.66 \mathrm{e}-09$ & 1.5 & 2.8 \\
0.646 & 10.715 & $1.45 \mathrm{e}-3$ & 0.69 & $1.49 \mathrm{e}-09$ & 1.0 & 3.4 \\
0.651 & 10.896 & $1.38 \mathrm{e}-3$ & 0.67 & $1.35 \mathrm{e}-09$ & 1.2 & 3.4 \\
0.657 & 11.078 & $1.42 \mathrm{e}-3$ & 0.73 & $1.31 \mathrm{e}-09$ & 1.8 & 3.4 \\
0.695 & 12.394 & $9.43 \mathrm{e}-4$ & 0.70 & $6.24 \mathrm{e}-10$ & 1.1 & 2.9 \\
0.700 & 12.575 & $8.71 \mathrm{e}-4$ & 0.68 & $5.52 \mathrm{e}-10$ & 1.3 & 2.9 \\
0.705 & 12.757 & $8.88 \mathrm{e}-4$ & 0.72 & $5.35 \mathrm{e}-10$ & 1.9 & 2.9 \\
\hline
\end{tabular}




\subsection{Comparison with World Data and Theoretical Cal- culations}

The main goal of this experiment is to resolve the $A(Q)$ discrepancies among experimental data of Mainz, Orsay and Saclay across the region $0.2<Q<0.4 \mathrm{GeV} / \mathrm{c}$. New $A(Q)$ data of this experiment are compared with world data at low energy transfer region in Fig. 6.15. This plot is already shown in section 1.3 and new data of this experiment are added. The $x$ axis is $Q$ in $\mathrm{GeV} / \mathrm{c}$ and the $y$ axis is the ratio of $A(Q)$ data over the fit function. The $x$ axis is changed to focus on small $Q^{2}$ region. New data of this experiment are marked with black star symbols. And old data are shown as blue circles(Mainz data), green triangles(Orsay data) and yellow diamonds(Saclay data). In the problematic region, new data show agreement with Saclay data within uncertainties. Data at the largest $Q$ value are closer to DESY data than Saclay data.

Fig. 6.16 compares new data with three theoretical calculations. Blue solid line and red dashed line are relativistic calculations of gross CIA [7] and Schiavilla [61]. They give good description over the whole $Q$ region. Red line is closer to Mainz data than new data of this experiment. Blue calculation lies between Mainz data and new data. It is considered that relativistic calculations need to be reexamined. The green short dashed line is from ChiPt calculation and it is limited in the region $Q<600 \mathrm{MeV}$. This is also closer to Mainz data. It is considered that the calculation of next order term is necessary. 


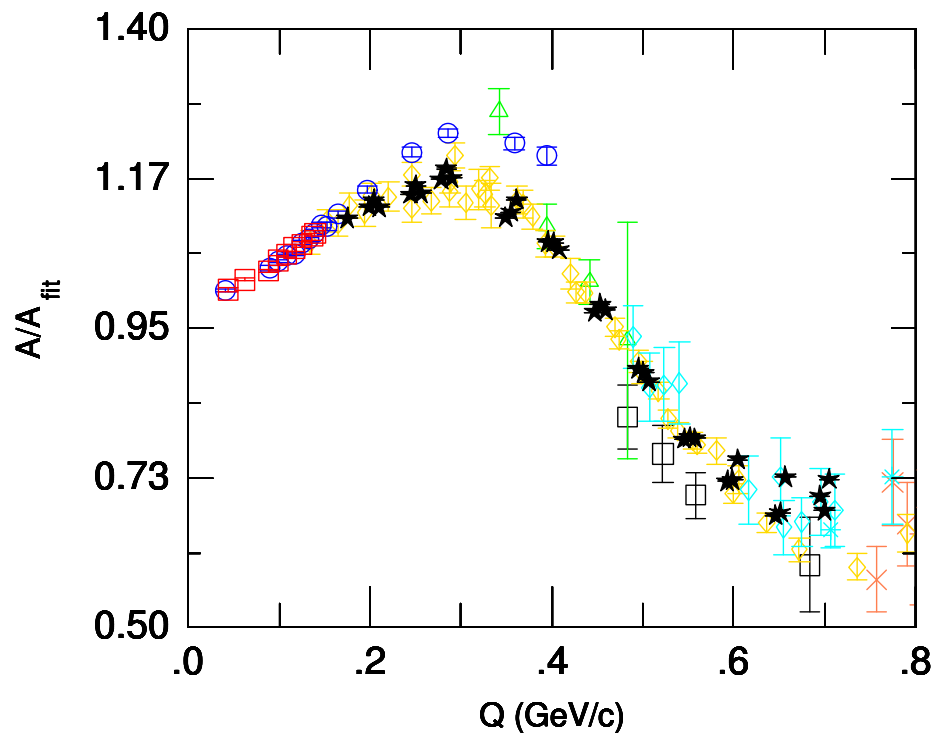

Figure 6.15: New data and world data.

\begin{tabular}{lccl} 
Experiment & $Q(\mathrm{GeV} / \mathrm{c})$ & Symbol & $\begin{array}{l}\text { Year and } \\
\text { Reference }\end{array}$ \\
\hline This & $0.18-0.71$ & $\star$ & 2009 \\
Experiment & & & \\
Monterey & $0.04-0.14$ & $\square$ & $1973[8]$ \\
Mainz & $0.04-0.39$ & $\bigcirc$ & $1981[9]$ \\
Saclay ALS & $0.13-0.84$ & & $1990[10]$ \\
Orsay & $0.34-0.48$ & $\triangle$ & $1966[11]$ \\
Stanford & $0.48-0.88$ & $\square$ & $1965[12]$ \\
DESY & $0.49-0.71$ & $\diamond$ & $1971[13]$ \\
CEA & $0.76-1.15$ & $\times$ & $1969[14]$ \\
SLAC E101 & $0.89-2.00$ & + & $1975[17]$ \\
\hline
\end{tabular}




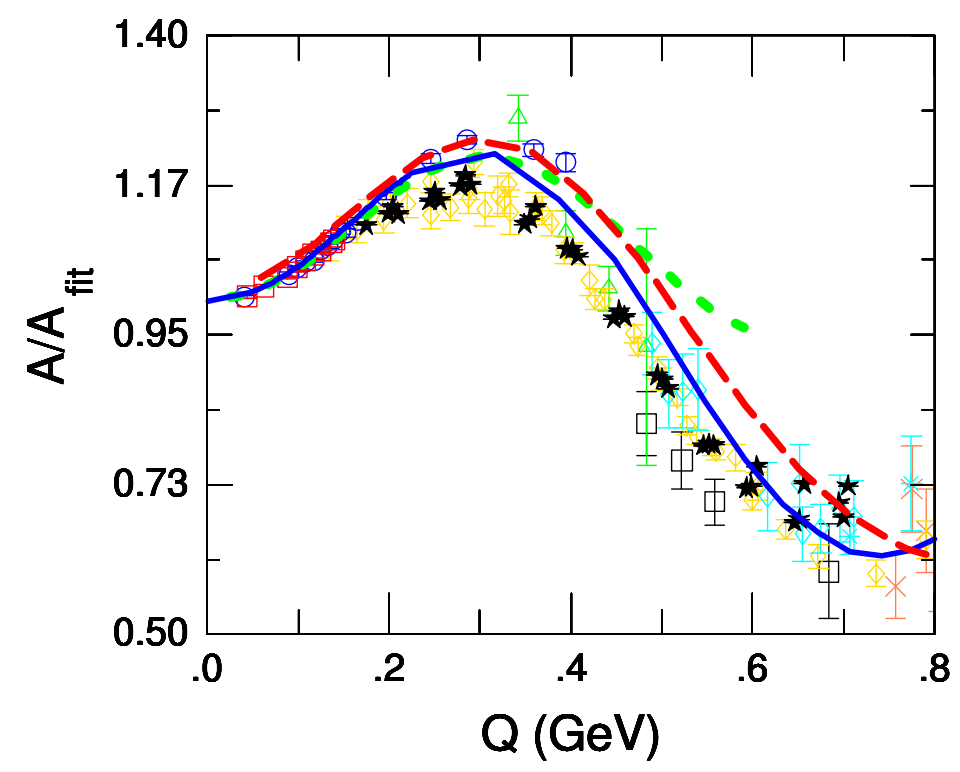

Figure 6.16: New data are compared with two relativistic calculations and ChiPT. The blue solid curve is the relativistic calculations from Gross CIA, the red dot curve is the relativistic calculations from Schivilla and the green dashed curve is the ChiPT from Phillips.

\begin{tabular}{lccl} 
Experiment & $Q(\mathrm{GeV} / \mathrm{c})$ & Symbol & $\begin{array}{l}\text { Year and } \\
\text { Reference }\end{array}$ \\
\hline This & $0.18-0.71$ & $\star$ & 2009 \\
Experiment & & & \\
Monterey & $0.04-0.14$ & $\square$ & $1973[8]$ \\
Mainz & $0.04-0.39$ & $\bigcirc$ & $1981[9]$ \\
Saclay ALS & $0.13-0.84$ & & $1990[10]$ \\
Orsay & $0.34-0.48$ & $\triangle$ & $1966[11]$ \\
Stanford & $0.48-0.88$ & $\square$ & $1965[12]$ \\
DESY & $0.49-0.71$ & $\diamond$ & $1971[13]$ \\
CEA & $0.76-1.15$ & $\times$ & $1969[14]$ \\
SLAC E101 & $0.89-2.00$ & + & $1975[17]$ \\
\hline
\end{tabular}




\section{Chapter 7}

\section{Conclusions}

Nucleon-nucleon interaction has been an attractive subject for a long time. Especially deuteron is the simplest nucleus and the only nucleon-nucleon bound system. It is believed that the study of deuteron can enhance the understanding of nucleonnucleon interaction.

Among the three structure functions of deuteron, $A(Q)$ shows the best agreement with theoretical calculations. The best calculations of $A(Q)$ still have differences of maximum $\sim 30 \%$ and the descriptions in high $Q$ region are still incomplete. There are several calculations with $\sim 10 \%$ discrepancy among them in the region $0.2<Q<0.4 \mathrm{GeV} / \mathrm{c}$ where relativistic effects start to become prominent. This difference becomes much bigger in high $Q$ region where relativistic effects are dominant. Hence obtaining accurate values in the low $Q$ region is important to make better calculations across the whole region and this should be resolved by experimental data. Nonetheless, there were discrepancies larger than $10 \%$ among experimental data of Mainz, Orsay and Saclay in the region. And they cannot even determine which is the correct sign of the relativistic effects.

In this circumstances, this experiment has been carried out to resolve the discrepancies among the existing experimental data. The elastic scattering of electron 
and deuteron is measured in Jefferson Lab Hall A using high resolution spectrometers. Cross sections of ${ }^{2} \mathrm{D}\left(e, e^{\prime}\right)^{2} \mathrm{D}$ are obtained from the ratio to data of ${ }^{1} \mathrm{H}\left(e, e^{\prime}\right)^{1} \mathrm{H}$ across the region $0.15<Q<0.7 \mathrm{GeV} /$ c. Instead of the Rosenbluth separation method which can produce both of $A(Q)$ and $B(Q)$ data directly, a new fit function of $B(Q)$ data is made and is subtracted from cross section data. In addition, kinematic settings are selected to suppress the $B(Q)$ contribution to be less than 1 $\%$ in the problematic region. Hence accurate $A(Q)$ data has been extracted with statistical uncertainties $0-2 \%$ and systematic uncertainties $1-3 \%$.

New data are compared with world data and they show good agreement with Saclay data within uncertainties in the problematic region of $0.2<Q<0.4 \mathrm{GeV} / \mathrm{c}$. They are compared with theoretical calculations. Some relativistic calculations agree with new data but they need some corrections to describe the high $Q$ region. And some relativistic calculations which give good description across the whole region are larger than new data. It is considered that they need to be reexamined. The ChiPT calculation is also compared. However it also gives predictions larger than new data. It is considered that next order calculations are required.

Recently new results for $\mathrm{G}_{\mathrm{Ep}}[62]$ is known that the value is approximately $2 \%$ smaller than the old one in the region of our interest. This form factor is used in all the calculations to describe the matrix element of deuteron current. $\left(\mathrm{G}_{E}^{p}\right)^{2}$ is proportional to $A(Q)$ and it is expected that $A(Q)$ values of all calculations will be reduced by approximately $\sim 4 \%$. Then, the CIA calculation, which gives the best description yet, can give good agreement with our new data. 


\section{Bibliography}

[1] R. Machleidt and I. Slaus, J. Phys. G 27, R69 (2001)

[2] J.J. Sakurai, Advanced Quantum Mechanics, Addison wesley (1967)

[3] F. Halzen and A. Martin, Quarks \& Leptons, John Wiley \& Sons (1984)

[4] R. Gilman and F. Gross, J. Phys. G 28, R37 (2002)

[5] M. Garçon and J. W. Van Orden, Advances in Nucl. Phys. 26, 293 (2001)

[6] I. Sick, Prog. Theo. Phys. 47, 245, 2001

[7] J. W. Van Orden, N. Devine and F. Gross, Phys. Rev. Lett. 75, 4369 (1995)

[8] R. W. Berard et al., Phys. Lett. B 47, 355 (1973)

[9] G. G. Simon et al., Nucl. Phys. A 364, 285 (1981)

[10] S. Platchkov et al., Nucl. Phys. A 510, 740 (1990)

[11] D. Benaksas et al., Phys. Rev. 148, 1327 (1966)

[12] C. D. Buchanan and R. Yearian, Phys. Rev. Lett. 15, 303 (1965)

[13] S. Galster et al., Nucl. Phys. B 32, 221 (1971)

[14] J. E. Elias et al., Phys. Rev. 177, 2075 (1969) 
[15] D. Abbott et al., Phys. Rev. Lett. 82, 1379 (1999)

[16] L. C. Alexa et al., Phys. Rev. Lett. 82, 1374 (1999)

[17] R. Arnold et al., Phys. Rev. Lett. 35, 776 (1975)

[18] D.R. Phillips, J. Phys. G 34, 365 (2007)

[19] R.B. Wiringa, V.G.J. Stoks, R. Schiavilla, Phys. Rev. C 51, 38 (1995)

[20] M. Lacombe et al., Phys. Rev. C 21, 861 (1980)

[21] R. Machleidt, K. Holinde and Ch. Elster, Phys. Reports 149, 1 (1987)

[22] J.L. Friar, Phys. Rev. C 12, 695 (1975)

[23] B.D. Keister ans W.N. Polyzou, Advances in Nucl. Phys. 20, 225 (1991)

[24] R. Schiavilla and D.O. Riska, Priv. Comm.

[25] J.L. Forest and A. Arriaga, Phys. Rev. C 60, 014002 (1999)

[26] R. Schiavilla and V.R. Pandharipande, Phys. Rev. C 65, 064009 (2002)

[27] F. Gross, J.W. Van Orden and K. Holinde, Phys. Rev. C 45, 2094 (1992)

[28] J.W. Van Orden, N. Devine and F. Gross, Phys. Rev. Lett. 75, 4369 (1995)

[29] H. Arenhövel, F. Ritz and T. Wilbois, Phys. Rev. C 61, 034002 (2000)

[30] T. W. Allen, W. H. Klink and W. N. Polyzou, Phys. Rev. C 63, 034002 (2001)

[31] J. Carbonell and V. A. Karmanov, Eur. Phys. J A 6, 9 (1999)

[32] F. M. Lev, E. Pace and G. Salmé, Phys. Rev. C 62, 064004 (2000)

[33] D. R. Phillips, S. J. Wallace and N. K. Devine, Phys. Rev. C 58, 2261 (1998) 
[34] F. Gross, Relativistic Quantum Mechanics and Field Theory, Wiley-Interscience (1993)

[35] C.W. Leemann, D.R Douglas and G.A. Krafft, Ann. Rev. Nucl. Part. Sci. 51, $413(2001)$

[36] J. Alcorn et al., Nucl. Instrum. Methods A 522, 294 (2004)

[37] Jefferson Lab Hall A homepage, http://hallaweb.jlab.org/

[38] A. Ketikyan et al., Lead Glass Total Absorption Shower Detector, http://hallaweb.jlab.org/equipment/detectors/detectors.html

[39] J. Berthot and P. Vernin, Nucl. Phys. News 9, 12 (1990)

[40] O. Ravel, Ph.D. thesis, University of Blaise Pascal, Clermont-Ferrand (1997)

[41] Hall A Analyzer BPM page, http://hallaweb.jlab.org/root/doc/bpm.html

[42] Jefersson Lab Proposal E04-005, J.-P. Chen et al.

[43] Jefferson Lab CODA homepage, http://coda.jlab.org/

[44] Hall A C ++ Analyzer homepage, http://hallaweb.jlab.org/root/index.html

[45] Hall A Raw data structure, http://hallaweb.jlab.org/equipment/daq/dstruct.html

[46] F. Benmokhtar, Ph. D. thesis, Rutgers University (2004)

[47] W. R. Leo, Techniques for Nuclear and Particle Experiments, Springer-Verlag, Berlin (1987)

[48] Hall A DAQ page, http://hallaweb.jlab.org/equipment/daq/daq_trig.html

[49] J. Yuan, Ph. D. thesis, Rutgers University (2008)

[50] Y. Oh, priv. comm. 
[51] National NUclear Data Center, http://www.nndc.bnl.gov/

[52] Hall A MCEEP manual, http://hallaweb.jlab.org/software/mceep/mceep.html

[53] J. Arrington and I. Sick, Phys. Rev. C 76, 035201 (2007)

[54] E.A.J.M. Offermann, Phys. Rev. C 44, 1096 (1991)

[55] J. Schwinger, Phys. Rev. 75, 898 (1949)

[56] S. Penner, Nuclear Structure Physics, Proceedings pf the 18th Scottish Univ. Summer School in Physics, (1977)

[57] L.W. Mo and Y.S. Tsai, Rev. Mod. Phys., 41, 205 (1969)

[58] Review of particle physics, Phys. Lett. B 667, 1 (2008)

[59] G.R. Lynch and O.I. Dahl, Nucl. Inst.Meth. in Phys. Res. B58, 6 (1991)

[60] http://hallaweb.jlab.org/news/minutes/tranferfuncs.html

[61] R. Gilman and R. Schiavilla, priv. comm.

[62] R. Gilman and X. Zhan, priv. comm. 


\section{초록}

\section{이병욱 \\ 물리천문학부 \\ 서울대학교 대학원}

이전에 수행되었던 낮은 운동량 전이에서의 전자-중성자 산란실험들 간에 는 차이점이 존재하는데, 이것으로 인해 포텐샬 모델들의 상대론적 효과 수정항의 부호를 결정하는 일과 카이랄 이론의 수렴성 판단이 어려운 상태 이다. 이 문제를 해결하기 위해서 제퍼슨 연구소에서 새로운 정밀 실험이 이루어졌다. $685 \mathrm{MeV}$ 의 에너지를 갖는 전자 빔으로 탄탈륨, 탄소, 수소, 중수소에 대해서 산란 측정이 이루어 졌다. 중수소 이외의 표적들은 실험 의 정밀도를 향상시키고, 산란단면적의 정확도를 확인하기 위하여 사용되 었다. 또한 4-운동량 전이 영역은 $0.15-0.7 \mathrm{GeV} / \mathrm{c}$ 에 달했다. 낮은 빔 전류의 정확한 측정을 위하여 새로운 장비인 실버 캘로리미터가 사용되 었고, 정확한 입사각을 알아내기 위해서 새로운 측정기 입구도 설치 되었 다.

수소 산란단면적과의 비례를 구함으로써 중수소 산란단면적을 구할 수 있 었다. 또한 $\mathrm{B}(\mathrm{Q})$ 함수에 대한 새로운 근사식을 만들고, 그 값을 산란단면적 으로부터 제거함으로써 $\mathrm{A}(\mathrm{Q})$ 함수를 구할 수 있었다. 정밀한 $\mathrm{A}(\mathrm{Q})$ 함수 값 들이 구하여 졌고, 이 논문에 그 값들이 수록되어 있다. 통계적 불확실성 은 $0.1-0.9 \%$ 에 이르고, 시스템 불확실성은 $1.2-2.9 \%$ 에 이른다. 이 실험 결과는 이전의 세클레이 실험결과와 일치함을 보이고 알려진 가장 정확한 이론 계산결과들보다는 약간 작은 값들을 가진다. 이론 계산들의 재점검이 필요한 것으로 보인다.

주요어 : 중수소, 산란단면적, 구조함수, $\mathrm{A}(\mathrm{Q})$, 전자-중성자 산란, 제퍼 슨 연구소

학번 : $2002-20398$ 



\section{감사의 글}

먼저 제 평생의 길을 인도해주시고 험난한 길 가운데서도 항상 형통케 하신 하나님께 감사와 영광을 드립니다.

이 논문이 나오기까지 정말 많은 분들의 도움이 있었습니다. 먼저, 제퍼슨 연구소에서 일할 수 있도록 도와주신 최선호 지도교수님께 진심으로 감사 드립니다. 막히는 문제가 있어서 찾아갈 때마다 개념과 원리에 대해 자세하게 설명해 주시고 바보 같은 질문을 해도 꾸짖지 않으시고 따뜻하게 답해 주셨습니다. 또한 논문심사 하는 동안 부족한 것들을 지적해 주시고 가르쳐주신 방형찬 교수님, 김수봉 교수님, 이원종 교수님, 김우영 교수님께도 감사의 말씀을 드립니다.

또한 제퍼슨 연구소의 연구책임자 Doug and Ron! 두 사람의 도움이 정말 컸습니다. 실험 초기 한참 동안 Ron을 실험 책임자인지도 모른 채 단지 관심 가지고 신경 써주는 고마운 사람이라고만 생각했던 것을 생각하면 웃음이 납니다. 형편없는 영어 때문에 반복해서 물어볼 때도 항상 친절하게 가르쳐 주고 따뜻하게 대해준 것들을 생각하면 정말 고마운 마음이 듭니다. (I wish to tender my gratitude from the bottom of my heart to Doug and Ron for their guidance, suggestions, comments and encouragement. I thank God for giving me the chance to study under them.

Ron, I did not know what the contact person is at the first. :)

수없이 귀찮게 했지만 항상 잘 가르쳐 준 Hall A의 맏형 Alex. Thank you so much . You are my hero -). Robert 와 Peter 에게도 Analyzer, ROOT, MCEEP 에 대해서 많은 도움을 받았습니다.

물리에 대해서 알게 해주신 민동필 교수님. 만날 때 마다 공부할 것들을 산더미처럼 던져 주시면서 격려해주시고 신경 써 주셨습니다. 게으르고 부족했지만, 교수님 덕분에 물리의 아름다운 모습들을 조금이나마 볼 수 있었습니다. 암센터 김종원 박사님께는 능동적으로 공부하는 자세를 배웠습니다. 박사님께 배우던 때가 정말 즐거웠고 더 일찍 시작하지 못한 것이 아쉬울 따름입니다. 
저와 함께 공부했던 대학원 식구들에게도 감사 드립니다. 이름이 같은 2 명의 병욱이 형들, 송박사님, 준일 형, 영호 형, 동호 형들과 함께 공부하던 때는 정말 즐거운 추억으로 남아있습니다. 같이 생활하며 여러 도움을 준 연구실 후배인 유민, 혜구, 정석, 호영 그리고 LEDEX 팀 동료인 Guy, Jackie, 동기인 현선, 용지이, 껀수, 철희, 도나, 진오에게 고마움을 전합니다. 항상 저를 아끼고 도와주신 많은 분들, 임마누엘 교회 목사님과 식구들에게도 감사함을 전합니다.

무엇보다 지금까지 저를 키우시고 헌신적인 사랑으로 지원해주신 아버지, 어머니. 어떻게 감사의 마음을 전해야 할지 모르겠습니다. 어느덧 부모가 되고 보니 그 은혜를 조금이나마 깨닫게 됩니다. 이 논문은 두 분의 사랑과 헌신으로 만들어 질 수 있었습니다. 부족한 사위 챙겨주시고 맛난 음식으로 힘을 북돋아 주시는 장인, 장모님께도 진심으로 감사 드립니다. 마지막으로 항상 곁에서 용기를 주고 신뢰해준 나의 사랑하는 아내 혜승이, 너무 이쁜 하나님의 선물 예현이. 두 사람은 저의 가장 큰 기쁨입니다. 